\title{
Nickel-Catalyzed Oxidative Decarboxylative (Hetero)Arylation Reactions
}

Aaron P. Honeycutt

West Virginia University, aphoneycutt@mix.wvu.edu

Follow this and additional works at: https://researchrepository.wvu.edu/etd

Part of the Inorganic Chemistry Commons, Medicinal-Pharmaceutical Chemistry Commons, and the Organic Chemistry Commons

\section{Recommended Citation}

Honeycutt, Aaron P., "Nickel-Catalyzed Oxidative Decarboxylative (Hetero)Arylation Reactions" (2018). Graduate Theses, Dissertations, and Problem Reports. 3754.

https://researchrepository.wvu.edu/etd/3754

This Dissertation is protected by copyright and/or related rights. It has been brought to you by the The Research Repository @ WVU with permission from the rights-holder(s). You are free to use this Dissertation in any way that is permitted by the copyright and related rights legislation that applies to your use. For other uses you must obtain permission from the rights-holder(s) directly, unless additional rights are indicated by a Creative Commons license in the record and/ or on the work itself. This Dissertation has been accepted for inclusion in WVU Graduate Theses, Dissertations, and Problem Reports collection by an authorized administrator of The Research Repository @ WVU.

For more information, please contact researchrepository@mail.wvu.edu. 


\title{
Nickel-Catalyzed Oxidative Decarboxylative (Hetero)Arylation Reactions
}

\author{
Aaron Honeycutt \\ Dissertation submitted to the Eberly College of Arts and Science \\ at West Virginia University \\ in partial fulfillment of the requirements \\ for the degree of \\ Doctor of Philosophy \\ in \\ Chemistry
}

\author{
Jessica Hoover, Ph.D., Committee Chairperson \\ Brian Popp, Ph.D. \\ Kung Wang, Ph.D. \\ Björn Söderberg, Ph.D. \\ Hanjing Tian, Ph.D.
}

\section{Department of Chemistry}
Morgantown, West Virginia 2018

Keywords: $\mathrm{C}-\mathrm{H}$ activation, decarboxylation, nickel, heteroaryl, phenanthridinones, nickel metallacycle

Copyright 2018 Aaron Honeycutt 


\title{
Abstract \\ Nickel-Catalyzed Oxidative Decarboxylative (Hetero)Arylation Reactions
}

\begin{abstract}
Aaron Honeycutt
Transition-metal-catalyzed decarboxylative coupling reactions have gained considerable attention over the past decade as an efficient route to form heterobiaryls. However, current methods for oxidative decarboxylative (hetero)arylation with unactivated $\mathrm{C}-\mathrm{H}$ bonds have been limited by poor substrate scope, control of regioselectivity, and chemospecificity. This thesis describes the development of a new nickel-catalyzed oxidative decarboxylative coupling (ODC) with unactivated $\mathrm{C}-\mathrm{H}$ bonds. The first chapter discusses the development of the new nickel-catalyzed ODC reaction to enable the coupling of a $\mathrm{N}, \mathrm{N}^{\prime}$-bidentate directing group with a broad scope of heteroaromatic carboxylates and ortho-substituted benzoates, a scope that has not been achieved in previous oxidative decarboxylative coupling transformations. The following chapter is an extension of the nickel-catalyzed ODC reaction for the synthesis of heterocycle-containing phenanthridinones. This chapter details an oxidative decarboxylative annulation with heteroaromatic carboxylates and ortho-fluorobenzoates. The final chapter describes the investigation and attempted synthesis of a proposed $\mathrm{Ni}$ (II) metallacycle from our catalytic cycles in the previous chapters. Synthesis of a $\mathrm{Ni}$ (II) metallacycle by denitrogenation of 3-(quinolin-8-yl)-3,4-dihydro-1,2,3-benzotriazin -4-one led to an interesting azanickelacycle dimer. However, decarbonylation of a 2(quinolin-8-yl)-2,3-dihydro-1H-isoindole-1,3-dione led to the desired five-membered metallacycle. Each of these chapters represents the challenges to developing an oxidative decarboxylative arylation with unactivated $\mathrm{C}-\mathrm{H}$ bonds with a first-row transition metal.
\end{abstract}




\section{Acknowledgments}

I would like to thank my advisor, Dr. Jessica M. Hoover for her guidance and giving me the opportunity to carry out my Ph.D. work under her supervision. Her strong commitment to the growth and support of her students has made my Ph.D. experience invaluable and unforgettable. Moreover, she has taught me to be diligent and meticulous in my studies and research. I will forever be indebted for her involvement in my life.

Additionally, I would like to thank my committee, Dr. Brian Popp, Dr. Kung Wang, Dr. Björn Söderberg, and Dr. Hanjing Tian for the valuable advice and suggestions on my research. I would also like to thank Dr. Jeffrey Petersen and Dr. Carsten Milsmann for their great help of the X-ray crystallography studies. In addition, I would like to thank Dr. Carsten Milsmann for always being around for help and guidance.

I would also like to thank all of my fellow colleagues, both past and present who have been an immense support while working in the lab and office. Thanks to Rob Crovak, Jiaqi Liu, Michael Stanton, Rebekah Krupa, John Riedesel, Lijun Chen, Lin Ju, Sierra Ciccone, Dr. Minghao Li, Dr. Anitha Shankara Linge Gowda, Dr. Bhasker Radaram, Dr. Kerry-Ann Green, Dr. Oliver Mitevski, and Prof. Andreas Baur. I would also like to thank the undergraduates who allowed me to teach and guide them through out their research. Thanks to Joseph Lokant, Mariah Murray, Ashley Moore, Justin Steets, Caitlin Embly, and Colin Siple.

In the end, I owe my deepest gratitude to my mother, wife, and family members. They have stood by my side and provided me with their continuous support and encouragement. Without their help, I would not have earned this degree. 


\section{Table of Contents}

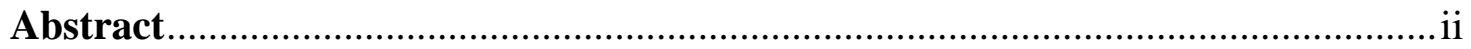

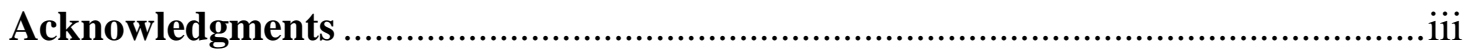

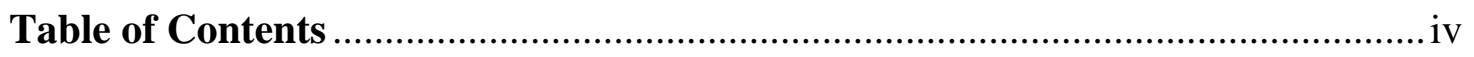

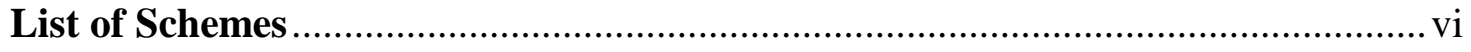

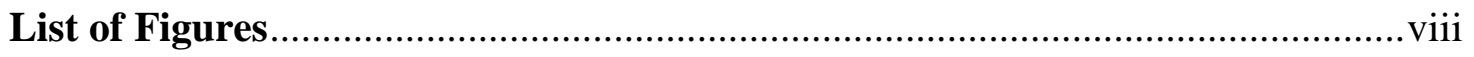

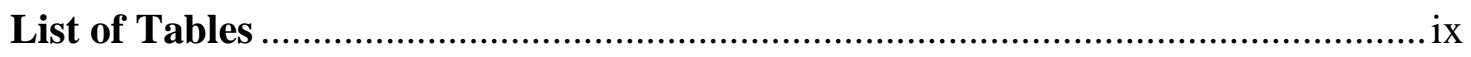

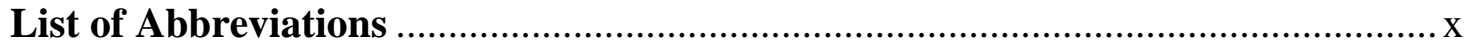

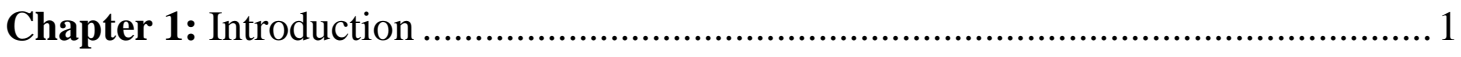

1.1. Traditional Cross-Coupling Systems for Unsymmetrical Biaryls ....................... 1

1.2. Decarboxylative Coupling Reactions for the Synthesis of Biaryls ..................... 2

1.3. Transition-Metal Catalyzed Directing Group Assisted C-H Activation .............. 8

Chapter 2: (Hetero)Arylation of Unactivated C-H Bonds by Oxidative Decarboxylative

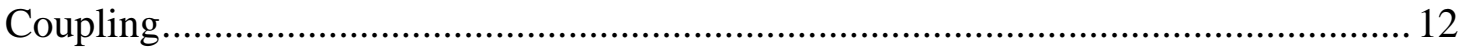

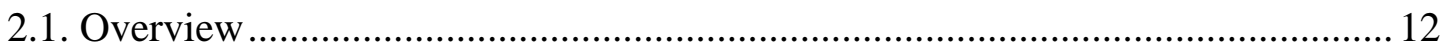

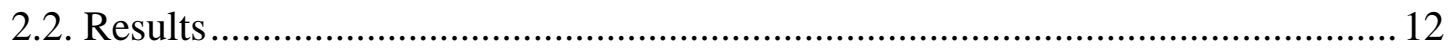

2.2.1. Condition Optimization for Nickel-Catalyzed Oxidative Decarboxylative

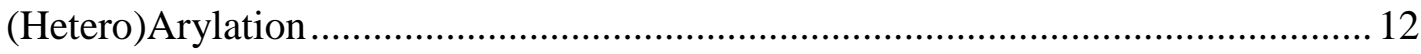

2.2.2. Exploration of the Nickel-Catalyzed Oxidative Decarboxylative

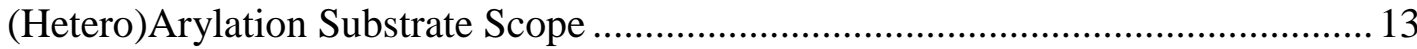

2.2.3. Preliminary Mechanistic Experiments of the Nickel-Catalyzed Oxidative

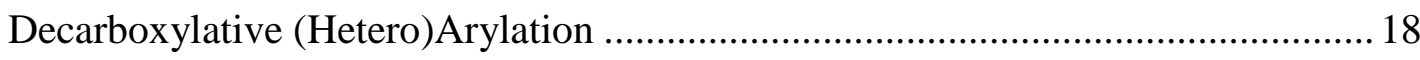

2.2.4. Proposed Reaction Mechanism of the Nickel-Catalyzed Oxidative Decarboxylative (Hetero)Arylation Reaction ................................................ 21

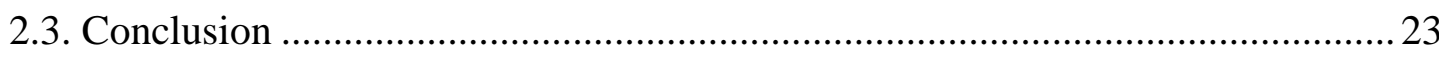

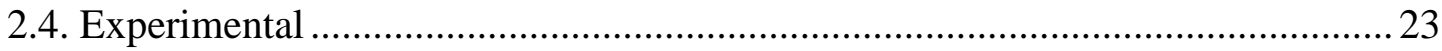


Chapter 3: A Nickel-Catalyzed Sequential Oxidative Decarboxylative (Hetero)Arylation and Cyclization for the Synthesis of Phenanthridinones 54

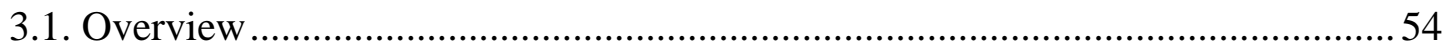

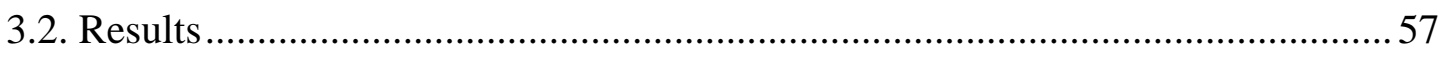

3.2.1. Optimization of the Sequential Oxidative Decarboxylative (Hetero)arylation and

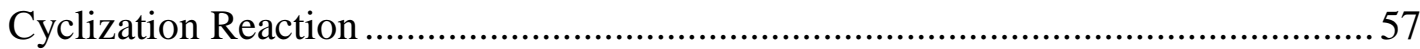

3.2.2. Scope of the Sequential Oxidative Decarboxylative (Hetero)arylation and

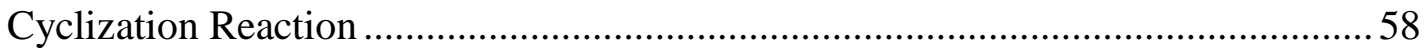

3.2.3. Preliminary Mechanistic Studies of the Sequential Oxidative Decarboxylative

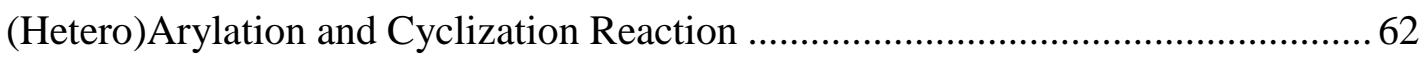

3.2.4. Proposed Reaction Mechanism of the Sequential Oxidative Decarboxylative

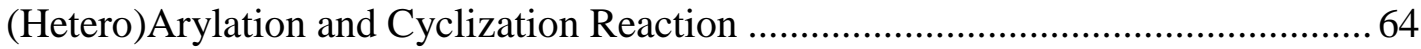

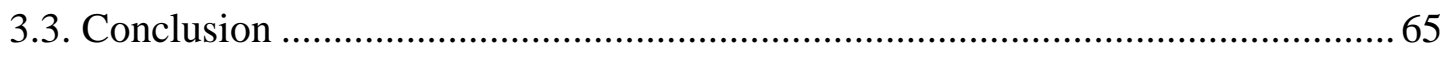

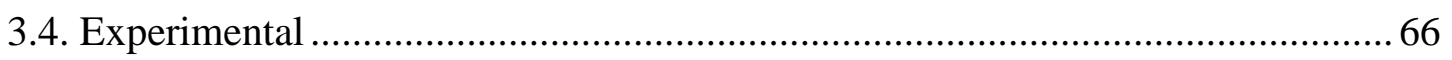

Chapter 4: Synthetic Routes to a Possible Nickel(II) Metallacycle: Denitrogenation and

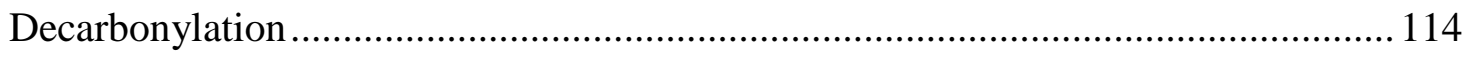

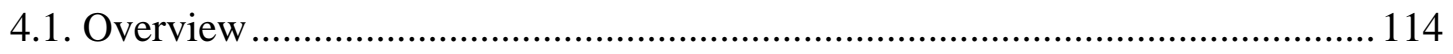

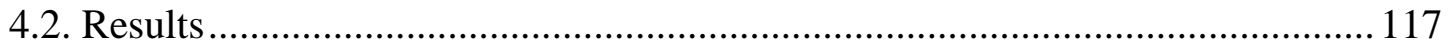

4.2.1. Synthesis of 1,2,3-Benzotriazine Derivative and Attempted Denitrogenation for

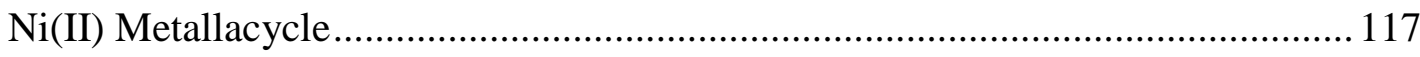

4.2.2. Synthesis of the $\mathrm{Ni}(\mathrm{II})$ metallacycle by Decarbonylation of Phthalimides... 122

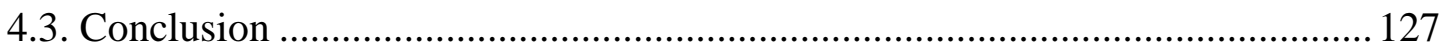

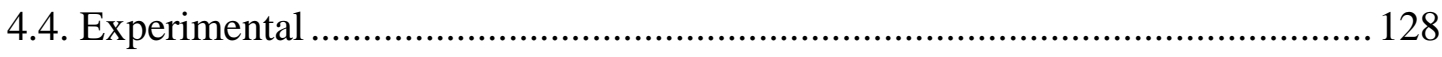

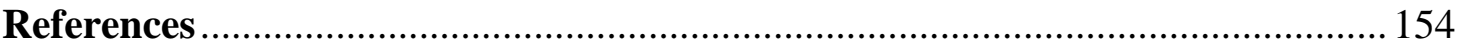




\section{List of Schemes}

Scheme 1. Traditional transition-metal-catalyzed cross-coupling for the synthesis of biaryls.

Scheme 2. Decarboxylative Ullmann coupling for the synthesis of biaryls with copper 3

Scheme 3. A bimetallic redox-neutral decarboxylative coupling of aryl halides with benzoic acids 3

Scheme 4. Oxidative decarboxylative couplings with activated $\mathrm{C}-\mathrm{H}$ bonds............4

Scheme 5. Selected examples of oxidative decarboxylative coupling reactions of

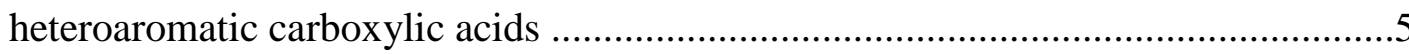

Scheme 6. Oxidative decarboxylative coupling with base metal catalysts ..............6

Scheme 7. Oxidative decarboxylative coupling with unactivated $\mathrm{C}-\mathrm{H}$ bonds ..........7

Scheme 8. Copper-mediated ODC reaction with unactivated $\mathrm{C}-\mathrm{H}$ bonds and 2nitrobenzoic acids

Scheme 9. C-H activation and functionalization with directing groups and a transition metal.

Scheme 10. First reported nickel metallacycle by $\mathrm{C}-\mathrm{H}$ activation ..........................

Scheme 11. Bidentate $\mathrm{C}-\mathrm{H}$ activation and functionalization with a

transition metal 10

Scheme 12. Selected examples of nickel-catalyzed directed $\mathrm{C}-\mathrm{H}$ functionalization reactions

Scheme 13. The targeted ODC reaction of unactivated $\mathrm{C}-\mathrm{H}$ bonds with (hetero)aromatic acids and first-row transition metal catalyst

Scheme 14. Targeted goal of achieving an oxidative decarboxylative arylation with unactivated $\mathrm{C}-\mathrm{H}$ bonds

Scheme 15. Kinetic isotope effect of the nickel-catalyzed oxidative decarboxylative (hetero)arylation .21

Scheme 16. Proposed reaction mechanism of the nickel-catalyzed oxidative decarboxylative (hetero)arylation 
Scheme 17. Examples of heterocycle containing phenanthridinone syntheses.......56

Scheme 18. Oxidative decarboxylative phenanthridinone syntheses .....................57

Scheme 19. Targeted goal of achieving a sequential oxidative decarboxylative heteroarylation and cyclization with unactivated $\mathrm{C}-\mathrm{H}$ bonds

Scheme 20. Kinetic isotope effect of the nickel-catalyzed sequential oxidative decarboxylative

Scheme 21. Base promoted cyclization by $\mathrm{S}_{\mathrm{N}} \mathrm{Ar}$

Scheme 22. Proposed reaction mechanism of the sequential oxidative decarboxylative (hetero)arylation and cyclization reaction

Scheme 23. Ni(II)-mediated $\mathrm{sp}^{3} \mathrm{C}-\mathrm{H}$ activation of a tertiary urea moiety .... 114

Scheme 24. First reported transition-metal-mediated decarbonylation. 115

Scheme 25. Ni-catalyzed decarbonylation of unstrained ketones

and phthalimides

Scheme 26. Formation of a five-membered azanickelacycle by denitrogenation ..117

Scheme 27. Proposed synthesis of a Ni(II) metallacycle from phthalimide or 1,2,3benzotriazine

Scheme 28. Synthesis of 3-(quinolin-8-yl)-3,4-dihydro-1,2,3-benzotriazin-4-one from 8aminoquinoline and 2-nitrobenzoyl chloride

Scheme 29. Reactivity of complex 8a under the nickel-catalyzed oxidative decarboxylative (hetero)arylation reaction conditions

Scheme 30. Synthesis of 2-(quinolin-8-yl)-2,3-dihydro-1H-isoindole-1,3-dione (9a) from 8-aminoquinoline and phthalic anhydride

Scheme 31. Synthesis of an azaacylNi(II) complex (10a) from insertion of nickel into the phthalimide $\mathrm{C}-\mathrm{N}$ bond

Scheme 32. Reactivity of azaacylNi(II) complex 10a under the nickel-catalyzed oxidative decarboxylative (hetero)arylation reaction conditions.....

Scheme 33. Decarbonylation of the azaacylNi(II) metallacycle 10a to

complex 11a.

Scheme 34. Arylation of complex 11a with silver(I)-aryl for C-C bond formation. 


\section{List of Figures}

Figure 1. Phenanthridinones in medicine and natural products ..........................55

Figure 2. Perspective view of the molecular structure of $\left[\left(\mathrm{C}_{16} \mathrm{H}_{10} \mathrm{~N}_{4} \mathrm{O}\right) \mathrm{Ni}\right]_{2}(\mathbf{8 a})$ with the atom labeling scheme for the non-hydrogen atoms. The thermal ellipsoids are scaled to enclose $50 \%$ probability. 120

Figure 3. Heating of 8a in DMF- $d_{7}$ : Reaction conditions: 8a $(0.015 \mathrm{mmol})$ in DMF- $d_{7}$ $(0.90 \mathrm{~mL})$ with 1,3,5-trimethoxybenzene as an internal standard at $140{ }^{\circ} \mathrm{C}$ to $160{ }^{\circ} \mathrm{C}$. 122

Figure 4. Perspective view of the molecular structure of $\left(\mathrm{C}_{16} \mathrm{H}_{10} \mathrm{~N}_{2} \mathrm{O}\right) \mathrm{Ni}(\mathrm{CO})(\mathbf{1 1 a})$ with the atom labeling scheme for the non-hydrogen atoms. The thermal ellipsoids are scaled to enclose $50 \%$ probability. 126 


\section{List of Tables}

Table 2.1. Optimization for oxidative decarboxylative (hetero)arylation ...............13

Table 2.2. Heteroaromatic carboxylate scope of the nickel-catalyzed oxidative decarboxylative (hetero)arylation 15

Table 2.3. Heteroaromatic carboxylates that do not undergo oxidative decarboxylative coupling.

Table 2.4. Benzoate scope of the nickel-catalyzed oxidative decarboxylative (hetero)arylation 17

Table 2.5. Benzamide scope of the nickel-catalyzed oxidative decarboxylative (hetero)arylation

Table 2.6. Control experiments of the nickel-catalyzed oxidative decarboxylative (hetero)arylation

Table 2.7. Radical scavengers with the nickel-catalyzed oxidative decarboxylative (hetero)arylation .20

Table 3.1. Optimization of the nickel-catalyzed sequential oxidative decarboxylative (hetero)arylation .58

Table 3.2. Ni-catalyzed oxidative decarboxylative arylation and cyclization heteroaromatic carboxylate scope .59

Table 3.3. Ni-catalyzed oxidative decarboxylative arylation and cyclization benzoate scope .60

Table 3.4. Ni-catalyzed oxidative decarboxylative arylation and cyclization benzamide scope

Table 3.5. Radical scavengers with the nickel-catalyzed sequential oxidative decarboxylative (hetero)arylation and cyclization reaction .62

Table 4.1. Attempted synthesis of a Ni(II) metallacycle by denitrogenation 119 


\section{List of Abbreviations}

$\begin{array}{ll}\text { Å: } & \text { Angstrom } \\ \text { acac: } & \text { Acetylacetonate } \\ \text { AcOH: } & \text { Acetic acid } \\ \text { ATR: } & \text { Attenuated total reflection } \\ \text { BQ: } & 1,4 \text {-Benzoquinone } \\ \text { C: } & \text { Celsius } \\ \text { calcd: } & \text { Calculated } \\ \text { cat:: } & \text { Catalyst } \\ \text { COD: } & \text { Cyclooctadiene } \\ \text { Cp: } & \text { Cyclopentadienyl } \\ \text { Cy: } & \text { Cyclohexyl } \\ \text { DCM: } & \text { Dichloromethane } \\ \text { dcpe: } & \text { Bis(dicyclohexylphosphino)ethane } \\ \text { DG: } & \text { Directing group } \\ \text { DHA: } & 9,10 \text {-Dihydroanthracene } \\ \text { DMA: } & N, N \text {-dimethylacetamide } \\ \text { DMF: } & N, N \text {-dimethylformamide } \\ \text { DMSO: } & \text { Dimethyl sulfoxide } \\ \text { Dppbenz: } & 1,2 \text {-Bis(diphenylphosphino)benzene } \\ \text { Et: } & \text { Ethyl } \\ \text { equiv: } & \text { Equivalent } \\ \text { ESI: } & \text { Electrospray ionization } \\ \text { FT-IR: } & \text { Fourier transform infrared spectroscopy } \\ \text { g: } & \text { Gram } \\ \text { h: } & \text { Hour } \\ \text { Het: } & \text { Hetero } \\ \text { HRMS: } & \text { High-resolution mass spectrometry } \\ \text { IMes·HCl: } & 1,3 \text {-Bis(2,4,6-trimethylphenyl)imidazolium Chloride } \\ & \end{array}$




\begin{tabular}{ll} 
iPr: & Isopropyl \\
L: & Ligand \\
OAc: & Acetate \\
OTf: & trifluoromethylsulfonate \\
Me: & Methyl \\
MHz: & Megahertz \\
mol: & Mole \\
mp: & Melting point \\
m/z: & Mass to charge ratio \\
M.S.: & Molecular sieves \\
NFSI: & N-fluorobenzenesulfonimide \\
NMP: & N-methyl-2-pyrrolidone \\
NMR: & Nuclear magnetic resonance \\
ODC: & Oxidative decarboxylative coupling \\
PCy: & Tricyclohexylphosphine \\
Ph: & Phenyl \\
phen: & 1,10-Phenanthroline \\
PivOH: & Trimethylacetic acid \\
PPh $:$ & Triphenylphosphine \\
ppm: & Parts per million \\
Q: & Quinolyl \\
S: & Solvent \\
S-Phos: & 2-Dicyclohexylphosphino-2',6'-dimethoxybiphenyl \\
TBAI: & Tetrabutylammonium iodide \\
$t$ Bu-XPhos: & 2-Di-tert-butylphosphino-2',4',6'-triisopropylbiphenyl \\
TEA: & Triethylamine \\
TEMPO: & 2,2,6,6-tetramethyl-1-piperidinyloxy \\
TFA: & Trifluoroacetate \\
TfOH: & Trifluoromethanesulfonic acid \\
THF: & Tetrahydrofuran \\
\hline &
\end{tabular}


THP: Tetrahydropyran

TMSO: 3-(trimethylsilyl)-2-oxazolidin 
Chapter 1: Introduction

\subsection{Traditional Cross-Coupling Systems for Unsymmetrical Biaryls}

Transition-metal-catalyzed cross-coupling reactions have gained considerable attention over the past thirty years for the construction of unsymmetrical biaryls. ${ }^{1}$ These wellknown reactions such as the Suzuki-Miyaura, ${ }^{2}$ Kumada, Negishi, ${ }^{3}$ and Mizoroki-Heck ${ }^{4}$ coupling reactions were developed for building various unsymmetrical biaryls (Scheme 1). ${ }^{5}$ These reactions have allowed chemists to construct complex pharmaceutical building blocks, increase industrial process development, and advance material sciences. However, these traditional cross-coupling methods require prefunctionalized aryl halide or pseudohalide coupling partners thereby resulting in poor atom- and step-economy. On the contrary, decarboxylative coupling reactions for biaryl formation would eliminate the need for prefunctionalized aryl halide or pseudohalide coupling partners because, carboxylic acids are ubiquitous in nature, commercially available in a large scope, and easy to handle. 


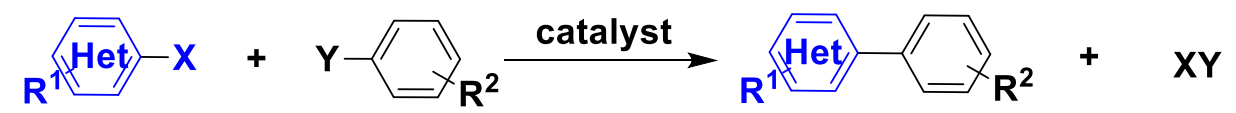

catalyst $=\mathrm{Pd}, \mathrm{Pt}, \mathrm{Cu}, \mathrm{Ni}$, etc .

$\mathrm{Y}=\mathrm{Br}$, I, OTf, etc.

$\mathrm{X}=\mathrm{Bpin}, \mathrm{Zn}, \mathrm{Mg}$, etc.

\section{Selected Examples}

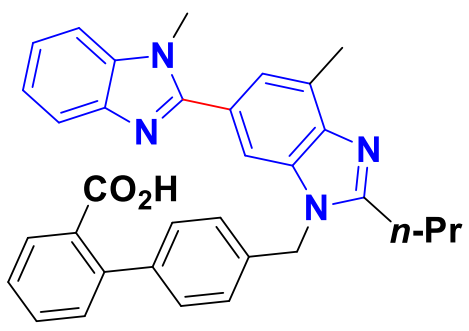

Telmisartan

(Micardis $^{\odot}$ )<smiles>O=C(Nc1ccccc1-c1ccc(Cl)cc1)c1cccnc1Cl</smiles>

Boscalid (BASF)<smiles>CC(Oc1cc(-c2cnn(C3CCN(C(=O)OC(C)(C)C)CC3)c2)cnc1N)c1c(Cl)ccc(F)c1Cl</smiles>

Crizotinib (Xalkori ${ }^{\circledR}$ ) $80 \%$

Scheme 1. Traditional transition-metal-catalyzed cross-coupling for the synthesis of biaryls.

\subsection{Decarboxylative Coupling Reactions for the Synthesis of Biaryls}

The first reported decarboxylative Ullmann-type coupling for the synthesis of biaryls was from Nilsson in $1966 .{ }^{6}$ Nilsson found that benzoic acids undergo decarboxylative coupling in the presence of stoichiometric amounts of copper and aryl iodides (Scheme 2). However, the reaction was limited to only ortho-nitrobenzoic acids and product yields were low due to harsh reaction conditions. However, this new method demonstrated the viability of using benzoic acids as nucleophilic coupling partners. 


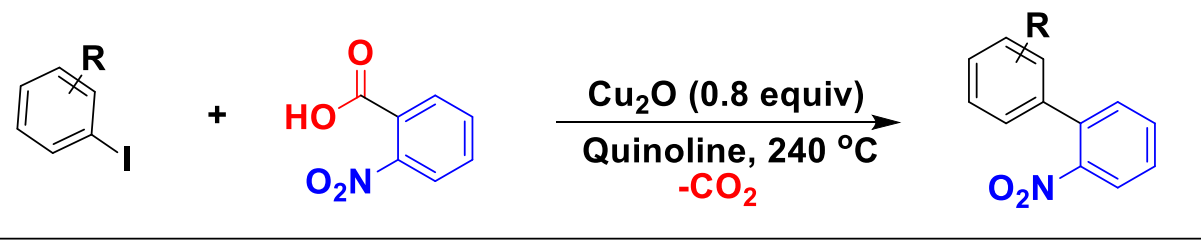

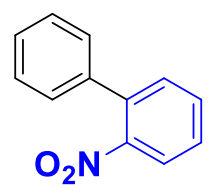

$10 \%$

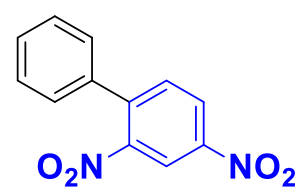

$16 \%$<smiles>COc1ccccc1-c1ccccc1[N+](=O)[O-]</smiles>

$50 \%$

Scheme 2. Decarboxylative Ullmann coupling for the synthesis of biaryls with copper.

The first catalytic decarboxylative cross-coupling reaction was not reported until forty years later. In 2006, Goossen and co-workers reported the first example of a bimetallic palladium/copper-catalyzed redox neutral decarboxylative cross-coupling of aromatic carboxylic acids with aryl halides (Scheme 3 ). ${ }^{7}$ This new method afforded unsymmetrical biaryls in good yields. However, this system is limited to ortho-substituted benzoic acids such as the 2-nitro, 2-fluoro, 2-methoxy, and 2-acetylbenzoic acids, for example.

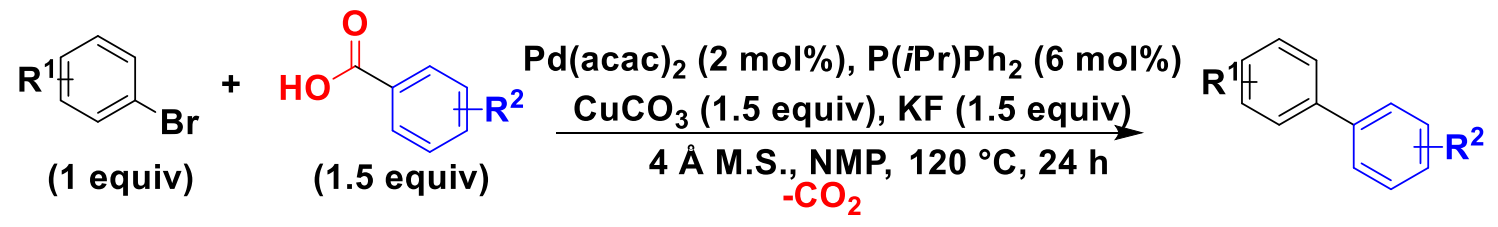<smiles>O=[N+]([O-])c1ccccc1-c1ccc(Cl)cc1</smiles>

$97 \%$<smiles>CC(=O)c1ccccc1-c1ccc(C)cc1</smiles>

$83 \%$<smiles>COc1ccccc1-c1ccccc1[N+](=O)[O-]</smiles>

$80 \%$<smiles>Cc1ccc(-c2ccccc2C=O)cc1</smiles>

$55 \%$<smiles>Cc1ccc(-c2ccccc2F)cc1</smiles>

$82 \%$<smiles>COc1ccccc1-c1ccc(C)cc1</smiles>

$29 \%$

Scheme 3. A bimetallic redox-neutral decarboxylative coupling of aryl halides with benzoic acids. 
Though Goossen and co-workers disclosed the first catalytic redox-neutral decarboxylative coupling, this reaction still requires the use of prefunctionalized aryl halides and pseudohalides as coupling partners. Efforts that are more recent have led to oxidative variants of these coupling reactions which would enable the coupling of arene $\mathrm{C}-\mathrm{H}$ bonds thereby eliminating prefuntionalized coupling partners. In 2008, Crabtree and co-workers reported the first Pd-catalyzed intermolecular and intramolecular decarboxylative arylation of $\mathrm{C}-\mathrm{H}$ bonds. ${ }^{8}$ Following this seminal work, other groups such as Larrosa ${ }^{9}$, $\operatorname{Tan}^{10}$, and $\mathrm{Su}^{11}$ have developed related oxidative decarboxylative coupling (ODC) reactions with $\mathrm{C}-\mathrm{H}$ bonds (Scheme 4) and have found that the strategies are typically limited in their substrate scope.

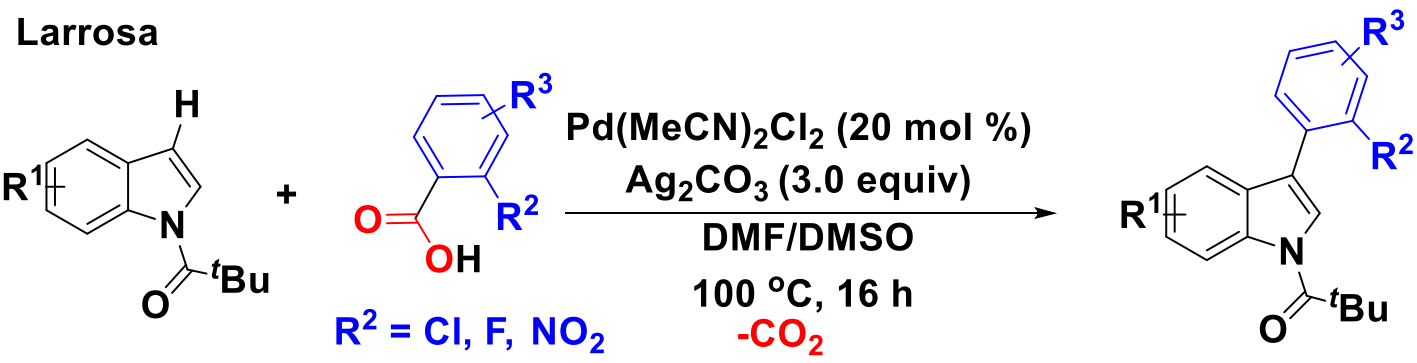

Tan

$\mathrm{PdCl}_{2}(20 \mathrm{~mol} \%)$

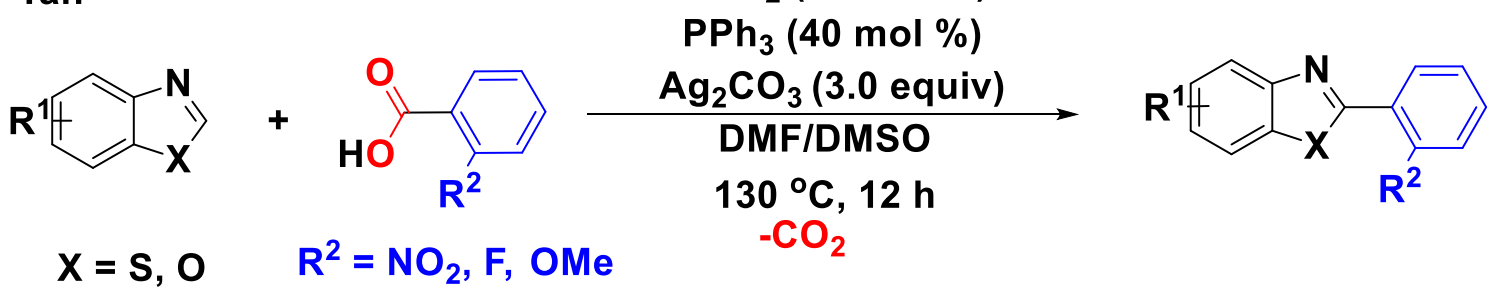

Su

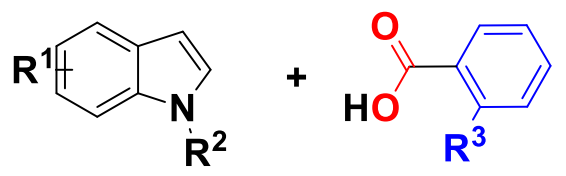

$\mathrm{R}^{2}=\mathrm{Ac}$, Piv $\quad \mathrm{R}^{3}=\mathrm{NO}_{2}, \mathrm{~F}, \mathrm{Cl}, \mathrm{OMe}$

$\operatorname{Pd}(\text { TFA })_{2}(7.5 \mathrm{~mol} \%)$

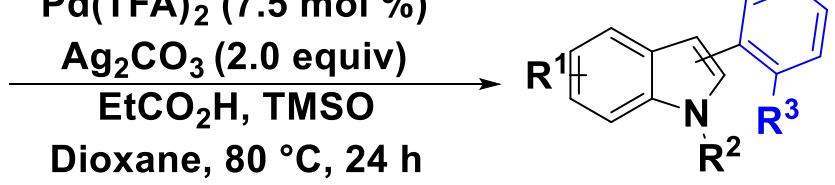

Scheme 4. Oxidative decarboxylative couplings with activated $\mathrm{C}-\mathrm{H}$ bonds.

The majority of oxidative decarboxylative couplings employ Pd catalysts. However, most of these catalyst systems are limited to ortho-substituted benzoic acids. The 
decarboxylative coupling of heteroaromatic carboxylic acids would be attractive as heterobiaryl structures are common motifs in pharmaceutically relevant compounds, ${ }^{12}$ and the corresponding esters are the direct products of traditional heterocycle syntheses. ${ }^{13}$ Notably, Greaney ${ }^{14}$ and $\mathrm{Su}^{15}$ reported the first ODC reactions of heteroaromatic acids; yet, they were limited to reactions with activated $\mathrm{C}-\mathrm{H}$ bonds such as oxazoles and thiophenes. A silver-mediated oxidative decarboxylative $\mathrm{C}-\mathrm{H}$ arylation that enables the coupling of pyridine carboxylic acids through a radical pathway has also been reported by $\mathrm{Su}$, but shows poor regioselectivity (Scheme 5). ${ }^{16}$

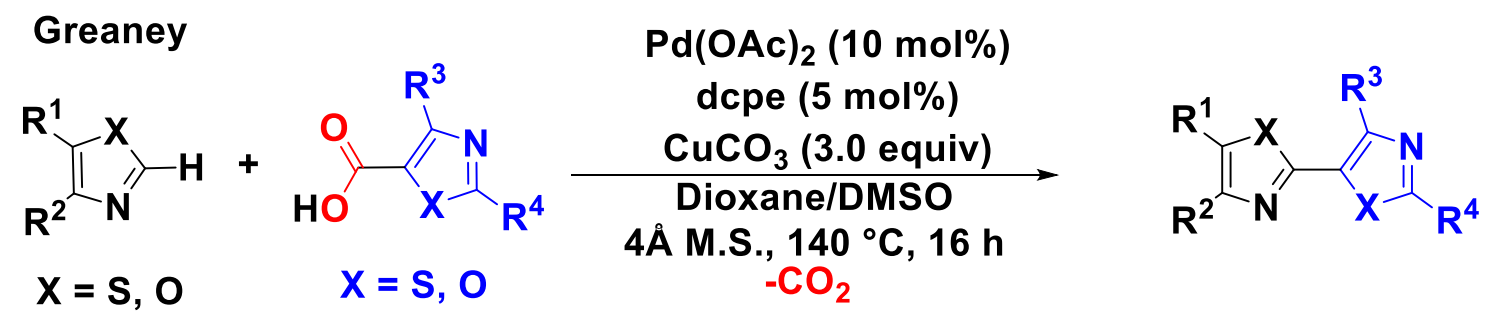

\section{Su}<smiles>[Y][R1]</smiles><smiles></smiles>

$\mathrm{X}=\mathrm{S}, \mathrm{O}$
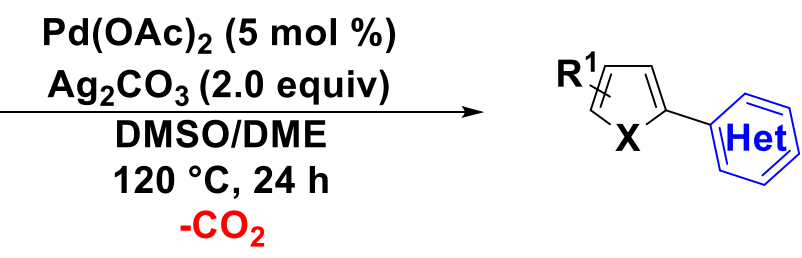

Su
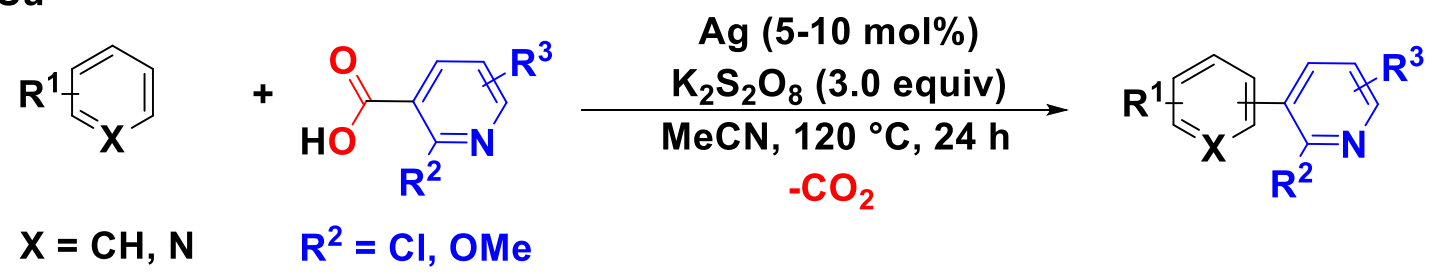

Scheme 5. Selected examples of oxidative decarboxylative coupling reactions of heteroaromatic carboxylic acids.

Recently, there has been interest in replacing noble-metal catalysts with base-metals in ODC reactions. However, many of these reactions that use copper- ${ }^{17}$ or nickel- ${ }^{42}$ catalysts are again limited to the coupling of 2-nitrobenzoic acids and pentafluorobenzoic acids with arenes bearing activated $\mathrm{C}-\mathrm{H}$ bonds. The one exception is a recent report of a copper-catalyzed ODC reaction from Shi and co-workers. ${ }^{18}$ They found their reaction 
method would couple 2-thiophene carboxylic acids. However, during their mechanistic studies they found the reaction proceeded through a protodecarboxylation and dehydrogenative coupling with complete loss of chemospecificity. Overall, these reaction methods that enable the oxidative decarboxylative heteroarylation of $\mathrm{C}-\mathrm{H}$ bonds with a base metal are limited to a specific class of heteroaromatics such as thiophene, azoles, and nicotinic carboxylic acids (Scheme 6).
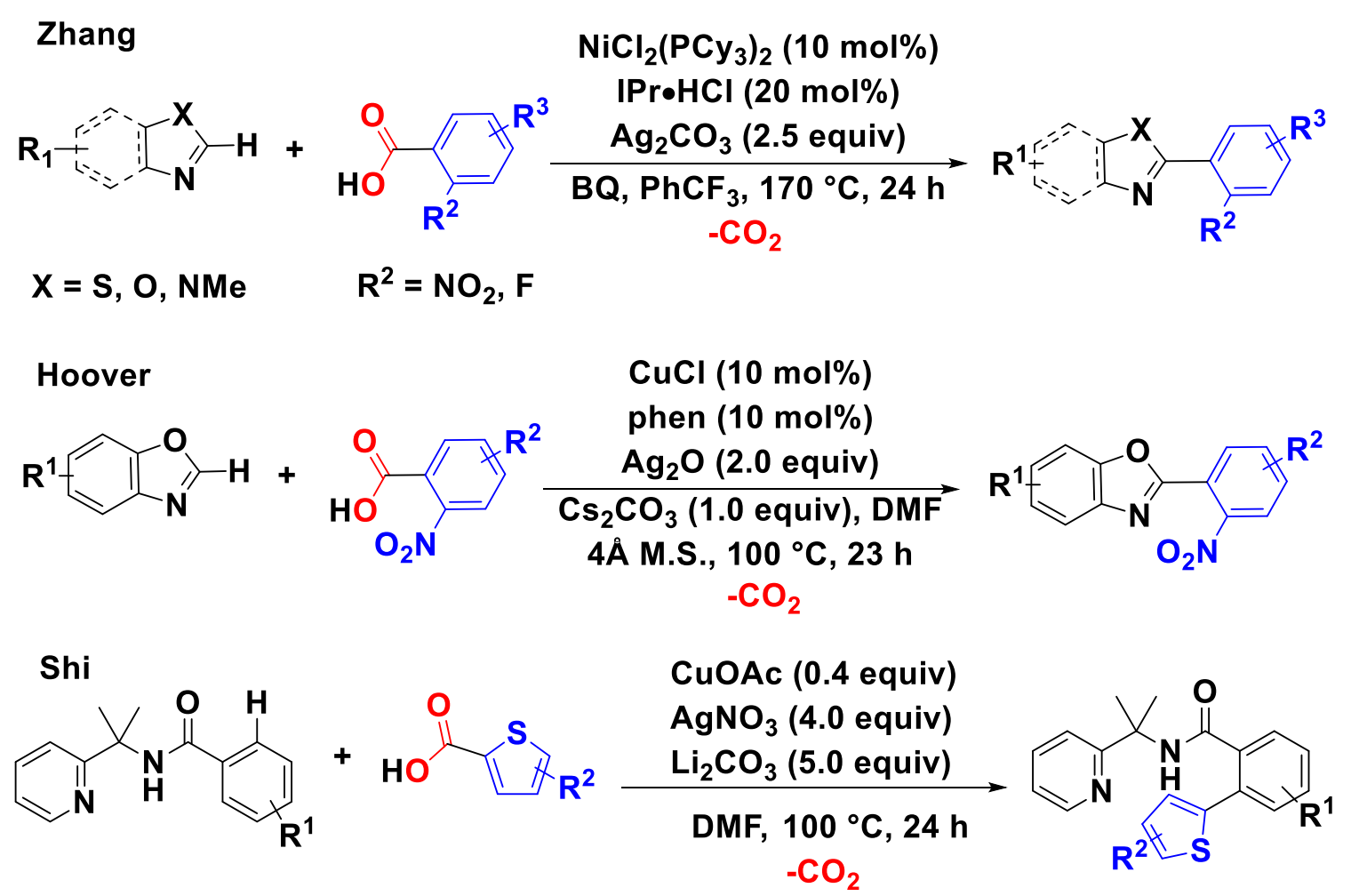

Scheme 6. Oxidative decarboxylative coupling with base metal catalysts.

Decarboxylative arylation reactions have similar limitations with respect to the $\mathrm{C}-\mathrm{H}$ coupling partner. As mentioned earlier, these reactions are often specific to couplings of electronically activated $\mathrm{C}-\mathrm{H}$ bonds such as oxazoles and thiazoles. Efforts to enable the decarboxylative arylation of unactivated $\mathrm{C}-\mathrm{H}$ bonds are limited. The Crabtree group ${ }^{8}$ and Glorius ${ }^{19}$ group reported early examples of oxidative decarboxylative coupling reactions with unactivated $\mathrm{C}-\mathrm{H}$ bonds; yet, both of these transformations form regioisomeric product mixtures. It was not until 2009 that $\mathrm{Yu}$ and co-workers reported the first $\mathrm{Pd}$ catalyzed decarboxylative arylation of arenes using 2-phenylpyridine and electron- 
deficient aryl acylperoxides (Scheme 7). ${ }^{20}$ Although this reaction allowed the installation of a broader scope of arenes not seen in previous ODC systems, the required aryl acyl peroxides are commercially limited and potentially dangerous.

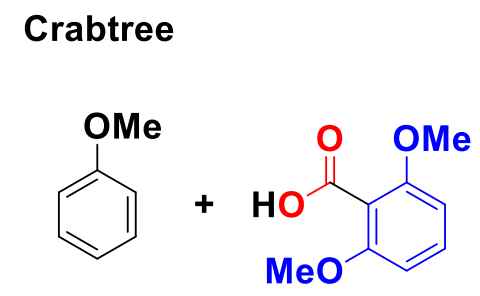

Yu<smiles>[R][R]c1ccc(C(=O)OOC(=O)c2ccc([R2])cc2)cc1</smiles>

$\mathrm{Pd}(\mathrm{OAc})_{2}(10 \mathrm{~mol} \%)$

tBu-XPhos (20 mol \%) $\mathrm{Ag}_{2} \mathrm{CO}_{3}$ (1.25 equiv)

4 Å M.S., DMF/DMSO microwave, $200{ }^{\circ} \mathrm{C}, 5 \mathrm{~min}$ $-\mathrm{CO}_{2}$
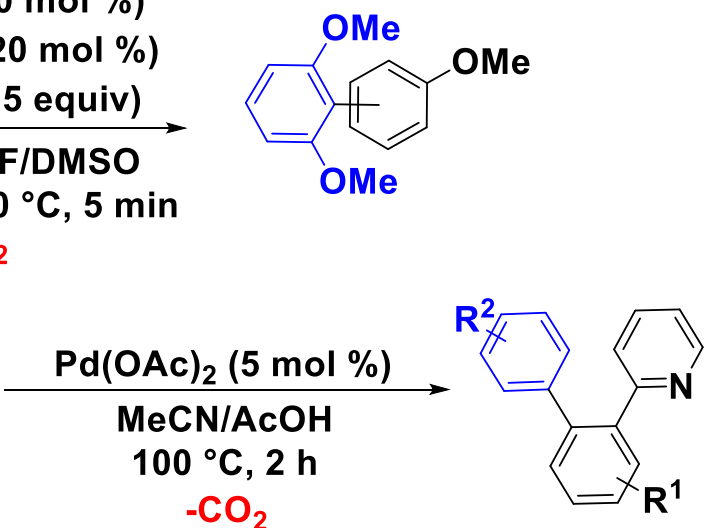

Scheme 7. Oxidative decarboxylative coupling with unactivated $\mathrm{C}-\mathrm{H}$ bonds.

Two copper systems have been reported recently that enable the decarboxylative coupling with arenes bearing benzamide directing groups. The first, from $\mathrm{Shi}^{18}$ and coworkers (Scheme 6), is specific to the coupling of 2-thiophene carboxylic acids through a tandem protodecarboxylation-dehydrogenative coupling pathway resulting in the loss of regiospecificity of the acids. More recently, Miura and co-workers have reported a copper-mediated ODC reaction of benzamides, however, their reaction suffers from limitations in the acid coupling partner, and efficient coupling is only achieved with 2nitrobenzoic acids (Scheme 8$){ }^{65}$ 


\section{Miura}

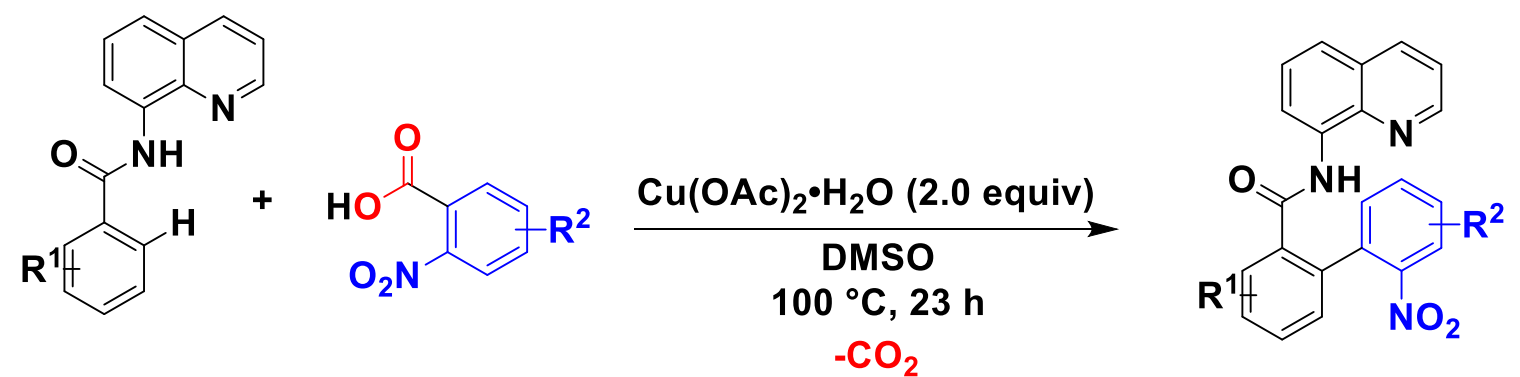

Scheme 8. Copper-mediated ODC reaction with unactivated $\mathrm{C}-\mathrm{H}$ bonds and 2nitrobenzoic acids.

There are currently no examples of a catalytic oxidative decarboxylative arylation of unactivated $\mathrm{C}-\mathrm{H}$ bonds capable of coupling a broad scope of (hetero)aromatic carboxylic acids. However, this thesis will later discuss the development of a new reaction method that overcomes these limitations by paring a base metal with a silver oxidant that will be discussed in Chapters 2 and 3.

\subsection{Transition-Metal Catalyzed Directing Group Assisted C-H Activation}

During the last decade, $\mathrm{C}-\mathrm{H}$ functionalization has been used as an invaluable technique for the installation of a plethora of functional groups in complex molecules. ${ }^{21}$ Due to the high bond-dissociation energy of a $\mathrm{C}-\mathrm{H}$ bond $\left(110 \mathrm{kcal} \mathrm{mol}^{-1}\right)$, transition metals are commonly used. The activation and functionalization of inert $\mathrm{C}-\mathrm{H}$ bonds with a transition metal offers an atom- and step-economical path towards building new methodologies in cross-coupling reactions. Recently, research groups have used the directing group (DG) approach for the activation of these inert $\mathrm{C}-\mathrm{H}$ bonds. In this approach, the transitional metal catalyst coordinates to the monodentate directing group, increasing the reactivity due to precoordination and facilitates the formation of a thermodynamically stable metallacycle (Scheme 9). ${ }^{22}$ This method has been applied not only to the formation of carbon-carbon bonds, but also carbon-heteroatom bonds. 


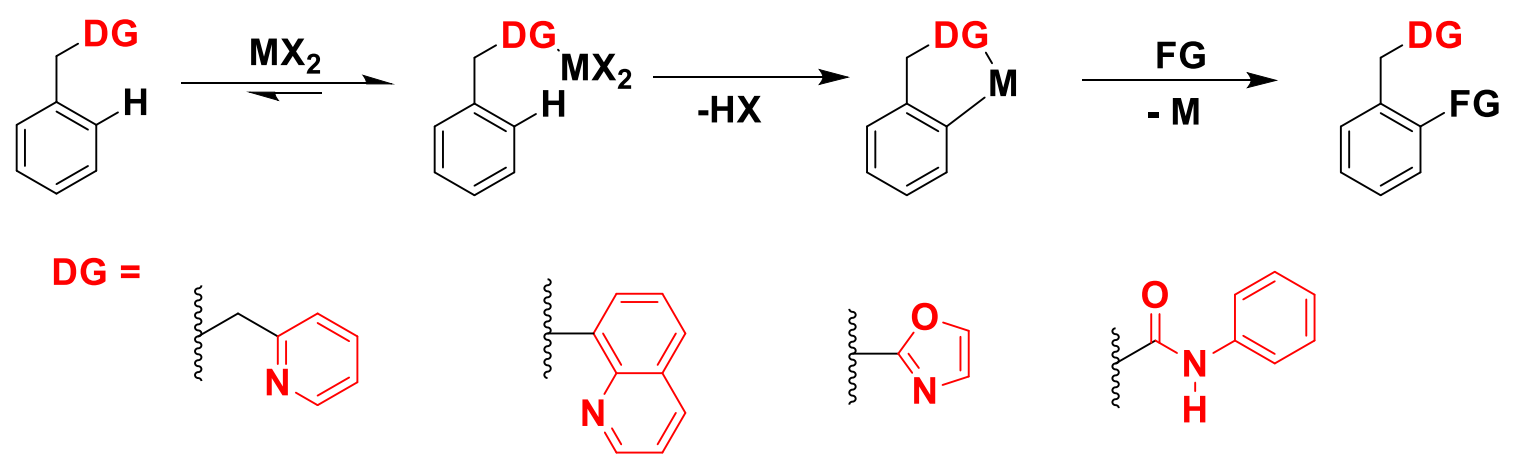

Scheme 9. C-H activation and functionalization with directing groups and a transition metal.

Early development of a directed cyclometalation of $\mathrm{C}-\mathrm{H}$ bonds with a group 10 metal was reported by Kleiman and Dubeck. ${ }^{23}$ They found that when $\mathrm{NiCp}_{2}$ as treated with azobenzene the formation of the cyclometalated complex cyclopentadienyl [o(phenylazo)phenyl]nickel resulted (Scheme 10, $\mathrm{Cp}=$ cyclopentadienyl). Following this work, Cope and co-workers reported a related cyclometalation of azo compounds using $\mathrm{PdCl}_{2}{ }^{24}$<smiles>c1ccc(N=Nc2ccccc2)cc1</smiles>
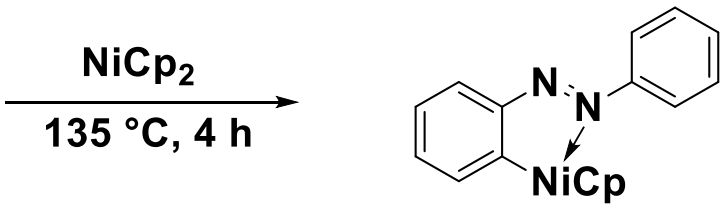

Scheme 10. First reported nickel metallacycle by $\mathrm{C}-\mathrm{H}$ activation.

Building on these early pioneering works, many groups have focused on developing new methodologies for $\mathrm{C}-\mathrm{H}$ functionalization using more sustainable and cost-effective first-row transition metals, such as $\mathrm{Co}, \mathrm{Ni}, \mathrm{Fe}$, and $\mathrm{Cu}$. However, there are fundamental challenges that first row transition metals face compared to their second and third row counter parts. First row transition metals have weaker M-C bonds making the catalytic system difficult to control upon $\mathrm{C}-\mathrm{H}$ activation. ${ }^{25}$ In addition, $\mathrm{C}-\mathrm{H}$ activation with first row-metals can be more complicated since these metals are prone to single electron oxidations.

To overcome these issues, many groups have used tethered bidentate directing groups made from benzoic acid derivatives with first row-metals. These bidentate directing 
groups allow for improved $\mathrm{C}-\mathrm{H}$ activation because: 1) they increase the reactivity because of the higher effective concentration of the catalyst at the site of interest 2) facilitate the formation of a thermodynamically stable metallacycle upon $\mathrm{C}-\mathrm{H}$ activation and 3) the metallacycle intermediate is stabilized in higher oxidation states after $\mathrm{C}-\mathrm{H}$ activation (Scheme 11) ${ }^{26,27}$

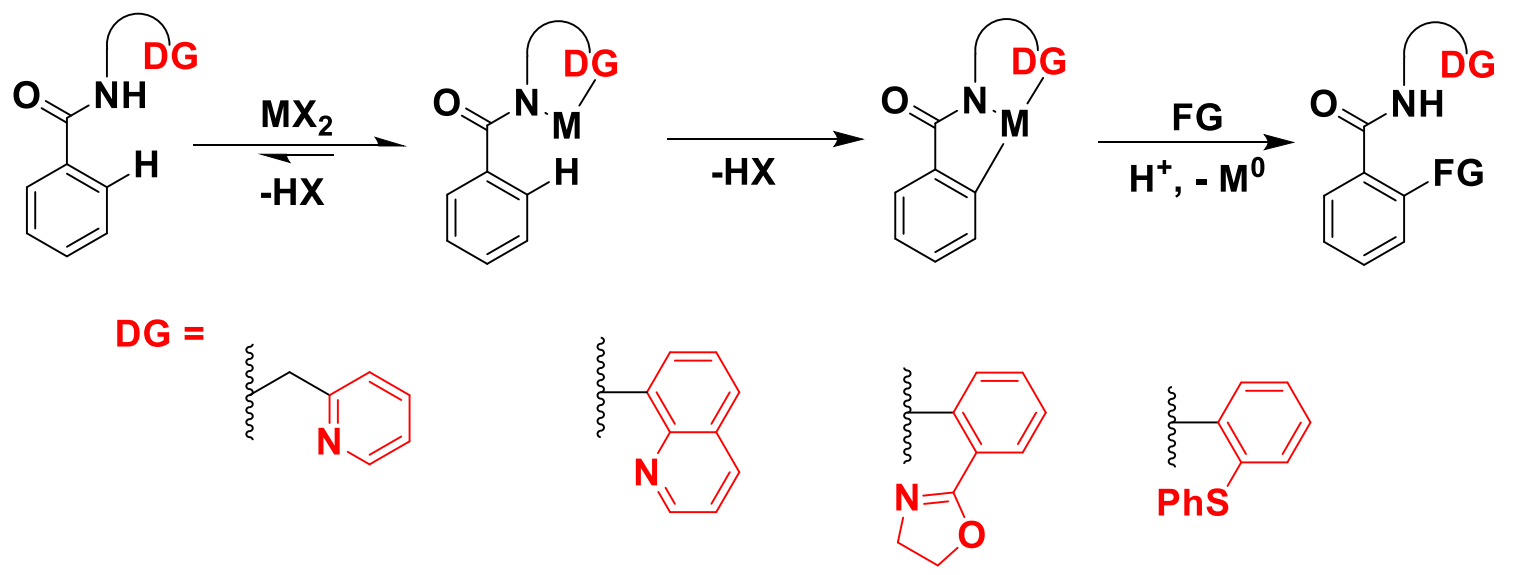

Scheme 11. Bidentate $\mathrm{C}-\mathrm{H}$ activation and functionalization with a transition metal.

Recently, nickel catalysis has received increasing attention due to its promising reactivity with $\mathrm{C}-\mathrm{H}$ bonds. This evidence has been shown in several examples in literature for forming $\mathrm{C}-\mathrm{C}^{32}, \mathrm{C}-\mathrm{N}^{28}$, and $\mathrm{C}-\mathrm{S}^{29}$ bonds utilizing a directing group. Initial progress in $\mathrm{C}-\mathrm{H}$ functionalization reactions catalyzed by nickel are limited to the activation and coupling of specific activated $\mathrm{C}-\mathrm{H}$ bonds such as polyfluorinated benzene, azoles, and pyridine derivatives. ${ }^{30}$ However, examples of nickel-catalyzed activation of unactivated $\mathrm{C}-\mathrm{H}$ bonds are limited. In 2011, Chatani and co-workers reported the first nickel-catalyzed functionalization of unactivated $\mathrm{C}-\mathrm{H}$ bonds using a $\mathrm{N}, \mathrm{N}^{\prime}$-bidentate directing group. ${ }^{31}$ This led to the development of a variety of different transformations such as, arylation, ${ }^{32}$ alkynylation, ${ }^{33}$ alkylation, ${ }^{34}$ halogenation, ${ }^{35}$ dehydrogenative coupling, ${ }^{36}$ and allylation (Scheme 12). ${ }^{37}$ 


\section{Chatani}<smiles>[R][R]C#C[R]</smiles>

$$
\begin{gathered}
\begin{array}{c}
\mathrm{Ni}(\mathrm{COD})_{2}(10 \mathrm{~mol} \%) \\
\mathrm{PPh}_{3}(40 \mathrm{~mol} \%)
\end{array} \\
\underset{\mathrm{PhCH}_{3}, 160{ }^{\circ} \mathrm{C}, 24 \mathrm{~h}}{\longrightarrow}
\end{gathered}
$$<smiles>[R]c1c([R])n(Cc2ccccn2)c(=O)c2ccc[R1]c12</smiles>

Shi<smiles>CC(C)(NC(=O)c1ccccn1)c1[R1]cccc1</smiles>

$+\quad$ LiX

$\mathrm{Ni}(\mathrm{OTf})_{2}$ (10 mol\%)

THP (20 mol\%)

$$
\begin{array}{cc}
\cline { 2 - 2 } & \mathrm{KMnO}_{4}\left(2.2 \text { equiv), } \mathrm{O}_{2}\right. \\
\mathrm{X}=\mathrm{Br}, \mathrm{I} & t-\mathrm{BuCN}, 140^{\circ} \mathrm{C}, 24 \mathrm{~h}
\end{array}
$$<smiles>[X]c1ccc[R1]c1C(=O)NC(C)(C)c1ccccn1</smiles>

You<smiles>[R1]c1ccccc1C(=O)Nc1cccc2cccnc12</smiles>
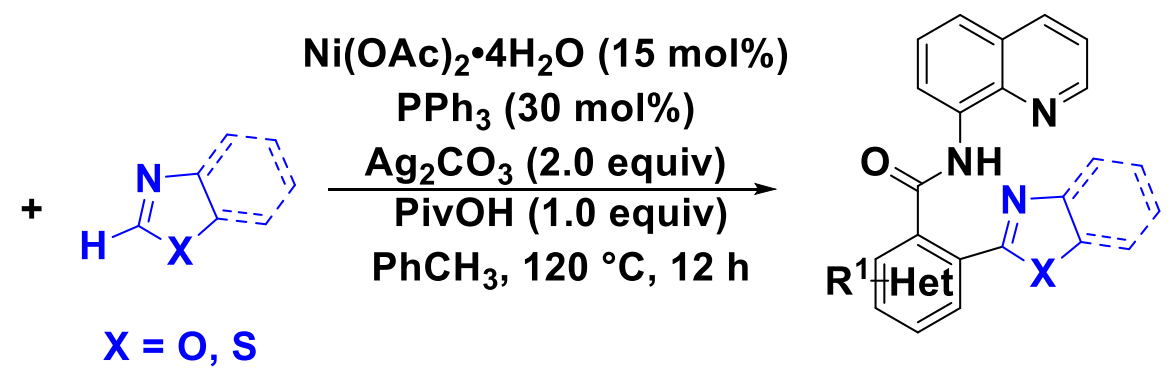

Scheme 12. Selected examples of nickel-catalyzed directed $\mathrm{C}-\mathrm{H}$ functionalization reactions.

We envisioned using a base metal in the presence of a $\mathrm{N}, \mathrm{N}^{\prime}$-bidentate directing group to help facilitate the functionalization of inert $\mathrm{C}-\mathrm{H}$ bonds for decarboxylative coupling. After many attempts, we finally succeed in developing a new method for the oxidative decarboxylative (hetero)arylation of unactivated $\mathrm{C}-\mathrm{H}$ bonds later discussed in the following chapters.

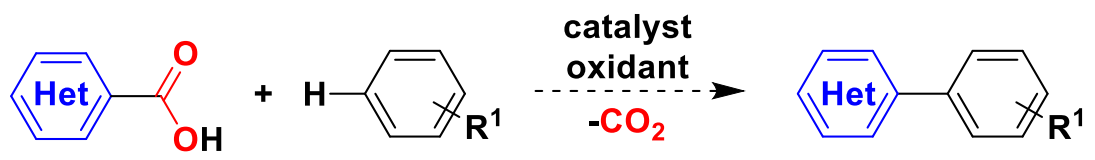

Scheme 13. The targeted ODC reaction of unactivated $\mathrm{C}-\mathrm{H}$ bonds with (hetero)aromatic acids and first-row transition metal catalyst. 
Chapter 2: (Hetero) Arylation of Unactivated C - H Bonds by Oxidative Decarboxylative Coupling

\subsection{Overview}

As seen in Chapter 1, oxidative decarboxylative couplings are becoming an attractive alternative to their redox-neutral counter parts. We initially set our goal of achieving an oxidative decarboxylative (hetero)arylation with unactivated $\mathrm{C}-\mathrm{H}$ bonds with heteroaromatic carboxylic acids, since, heterobiaryls can be found in many pharmaceuticals, agrochemicals, and material sciences. ${ }^{38} \mathrm{We}$ hypothesized that an unactivated $\mathrm{C}-\mathrm{H}$ bond could be functionalized with a heteroaromatic carboxylic acid in the presence of a base metal catalyst and oxidant (Scheme 14).

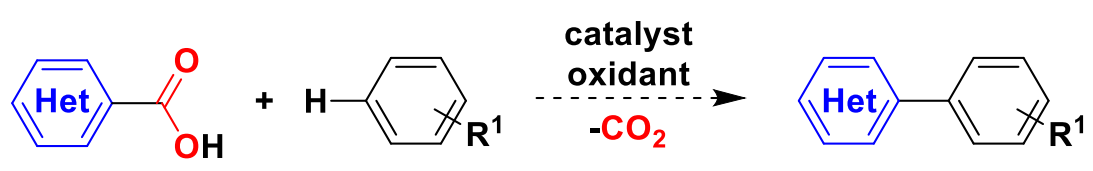

Scheme 14. Targeted goal of achieving an oxidative decarboxylative arylation with unactivated $\mathrm{C}-\mathrm{H}$ bonds.

\subsection{Results}

\subsubsection{Condition Optimization for Nickel-Catalyzed Oxidative Decarboxylative (Hetero)Arylation}

We initially designed our reaction using 2-methyl- $N$-(quinolin-8-yl)benzamide (1a) and 4-methyl-2-phenyl-1,3-thiazole-5-carboxylate (2a) in the presence of a catalyst and oxidant. We began using a copper catalyst since copper salts have been shown to undergo a directed $\mathrm{C}-\mathrm{H}$ arylation ${ }^{39}$ and enable the decarboxylation of a broad scope of benzoic acids. ${ }^{40}$ However, all attempts to use copper for this transformation were unsuccessful (Table 2.1 entry 1). Surprisingly, Ni salts were found to generate the desired ODC product in high yield when compared to copper (Table 2.1 entry 2). The use of $\mathrm{Ni}(\mathrm{OAc})_{2} \bullet 4 \mathrm{H}_{2} \mathrm{O}$ was found to be the most effective pre-catalyst which generated the desired ODC product in $45 \%$ yield (Table 2.1 entry 3 ). We then found that using pivalic acid as an additive increased the yield from $45 \%$ to $64 \%$ (Table 2.1 entry 5). Finally, 
when the loading of 4-methyl-2-phenyl-1,3-thiazole-5-carboxylate (2a) was lowered from 5 equiv to 3 equiv we were able to achieve quantitative yields of the desired ODC product. In contrast, when there is no nickel or silver present in the reaction, no desired product is formed (Table 2.1 entry 7 and 8). Therefore, our final optimized conditions consisted of $20 \mathrm{~mol} \%$ of $\mathrm{Ni}(\mathrm{OAc})_{2} \bullet 4 \mathrm{H}_{2} \mathrm{O}, \mathrm{Ag}_{2} \mathrm{CO}_{3}$ (2.0 equiv), $\mathrm{Na}_{2} \mathrm{CO}_{3}$ (4.0 equiv), and $\mathrm{PivOH}$ (1.5 equiv) in DMA at $110^{\circ} \mathrm{C}$ for $24 \mathrm{~h}$. This finding was quite interesting because nickel salts have been shown to be effective catalyst for directed $\mathrm{C}-\mathrm{H}$ activation. ${ }^{41}$ However, their use in oxidative decarboxylative arylations have been shown in only two previous examples, both of which are limited to the reactions of 2-nitro- and pentafluorobenzoic acids with activated $\mathrm{C}-\mathrm{H}$ bonds. ${ }^{42}$

Table 2.1. Optimization for oxidative decarboxylative (hetero)arylation ${ }^{a}$

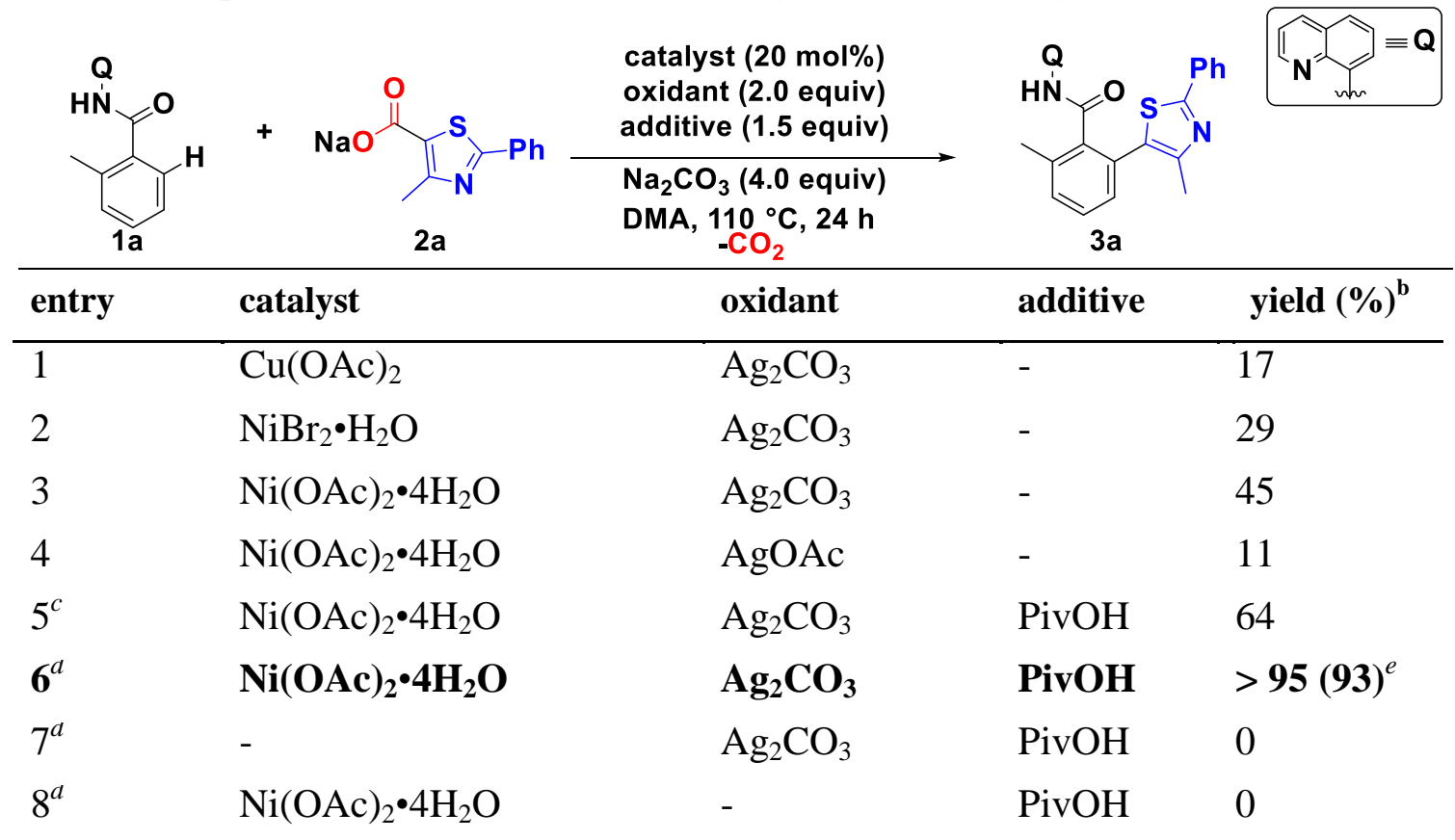

${ }^{a}$ Reaction conditions: Benzamide 1a $(0.2 \mathrm{mmol})$, heteroaromatic carboxylate 2a $(1.0 \mathrm{mmol})$ in DMA $(2 \mathrm{~mL}) .{ }^{b 1} \mathrm{H}$ NMR yield with 1,3,5-trimethoxybenzene as an internal standard. ${ }^{c}$ Pivalic acid $(0.3 \mathrm{mmol}){ }^{d}$ Heteroaromatic carboxylate $2 \mathbf{a}(0.6 \mathrm{mmol}){ }^{\mathrm{e}}$ Isolated yield

\subsubsection{Exploration of the Nickel-Catalyzed Oxidative Decarboxylative (Hetero)Arylation Substrate Scope}

Since heteroaromatic carboxylates were successfully coupled using this new Ni-catalyst system, we examined the scope of this new reactivity (Table 2.2). Thiazole derivatives 
bearing electron-withdrawing groups (3a, 3b, and 3d) gave higher yields than those bearing electron-donating groups (3c). Not only do thiazole carboxylates undergo oxidative decarboxylative coupling but also oxazoles in $74 \%$ yield (3e). The unsubstituted thiazole-5-carboxylate (3f) also undergoes coupling with complete chemoselectivity at the $\mathrm{C}-5$ position in $90 \%$ yield. We were encouraged to see this new method could also be extended to pyrazoles (3g and $\mathbf{3 h}$ ), triazole (3i), benzoazoles (3j and $3 \mathbf{k})$, and ortho-fluoro pyridine (3l). 
Table 2.2. Heteroaromatic carboxylate scope of the nickel-catalyzed oxidative decarboxylative (hetero)arylation ${ }^{a}$

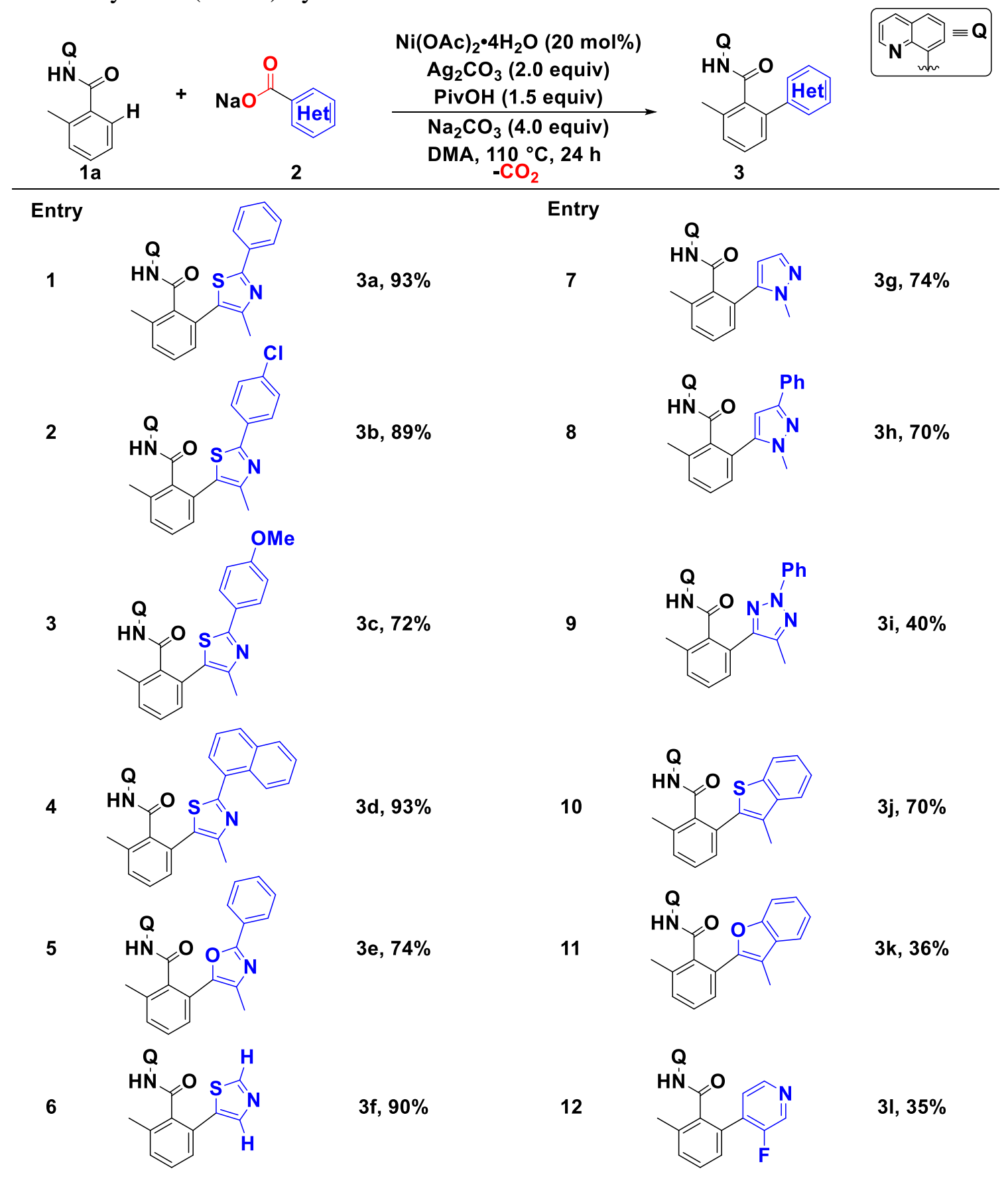

${ }^{a}$ Isolated yields. Reaction conditions: Benzamide $\mathbf{1 a}(0.2 \mathrm{mmol})$, heteroaromatic
carboxylate $\mathbf{2}(0.6 \mathrm{mmol}), \mathrm{Ni}(\mathrm{OAc})_{2} \bullet 4 \mathrm{H}_{2} \mathrm{O}(20 \mathrm{~mol} \%), \mathrm{Ag}_{2} \mathrm{CO}_{3}(2.0$ equiv $), \mathrm{Na}_{2} \mathrm{CO}_{3}(4.0$
equiv), and PivOH (1.5 equiv) in DMA $(2 \mathrm{~mL})$ for $24 \mathrm{~h}$ at $110^{\circ} \mathrm{C}$ under a $\mathrm{N}_{2}$ atmosphere. 
However, other pyridine carboxylates such as picolinic carboxylate, picolonic carboxylate $\mathrm{N}$-oxide, and 1-isoquinoline carboxylate did not undergo decarboxylative coupling (Table 2.3). This could be due to the thermal instability of the pyridine derivatives, which are known to undergo thermal decarboxylation at lower temperature than our reaction conditions. ${ }^{43}$ These data show pyridine derivatives with orthocarboxylates were not able to undergo cross-coupling.

Table 2.3. Heteroaromatic carboxylates that do not undergo oxidative decarboxylative coupling ${ }^{a, b}$

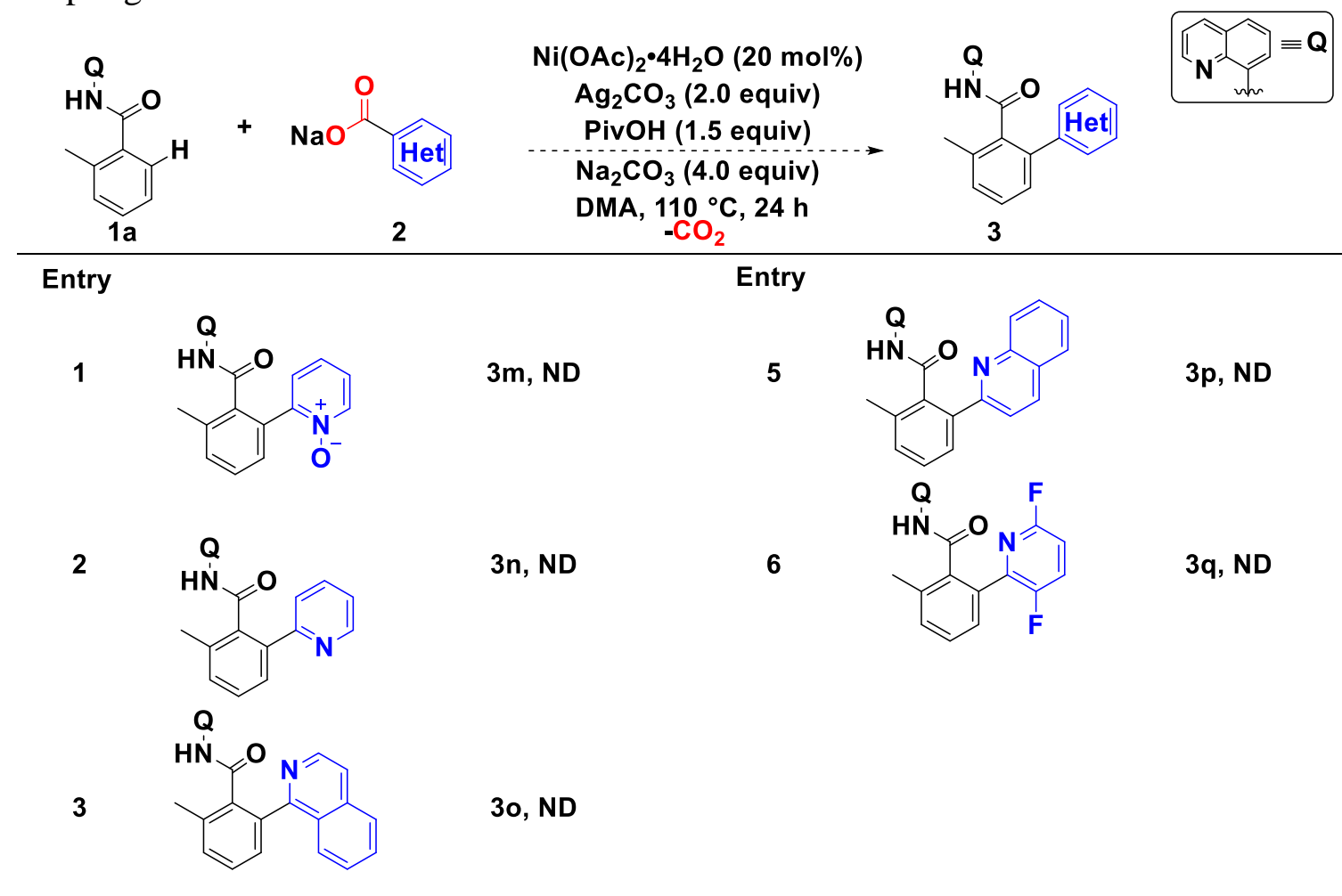

${ }^{a}$ Reaction conditions: Benzamide 1a $(0.2 \mathrm{mmol})$, heteroaromatic carboxylate $2(0.6 \mathrm{mmol})$, $\mathrm{Ni}(\mathrm{OAc})_{2} \bullet 4 \mathrm{H}_{2} \mathrm{O}(20 \mathrm{~mol} \%), \mathrm{Ag}_{2} \mathrm{CO}_{3}$ (2.0 equiv), $\mathrm{Na}_{2} \mathrm{CO}_{3}$ (4.0 equiv), and $\mathrm{PivOH}$ (1.5 equiv) in DMA ( $2 \mathrm{~mL})$ for $24 \mathrm{~h}$ at $110{ }^{\circ} \mathrm{C}$ under a $\mathrm{N}_{2}$ atmosphere. ${ }^{b} \mathrm{ND}=$ None detected by ${ }^{1} \mathrm{H}$ NMR with 1,3,5-trimethoxybenzene as an internal standard.

Next, we wanted to demonstrate that our new Ni-catalyst system was not limited to only coupling heteroaromatic carboxylates. We then investigated the reactivity of benzoates under the optimized reaction conditions, (Table 2.4). Decarboxylative arylation of the ortho-substituted benzoates proceeded cleanly with electron-donating and electronwithdrawing substitutes in varying positions. As shown in Table 2.4, 2-fluorobenzoates 
provided the highest yields $(\mathbf{3 r}, \mathbf{3 s}, \mathbf{3 t}$, and $\mathbf{3 u})$ over other ortho-substituted benzoates. It should be noted that arylbromides and chlorides are tolerated under these reaction conditions allowing for further functionalization.

Table 2.4. Benzoate scope of the nickel-catalyzed oxidative decarboxylative (hetero)arylation ${ }^{a}$

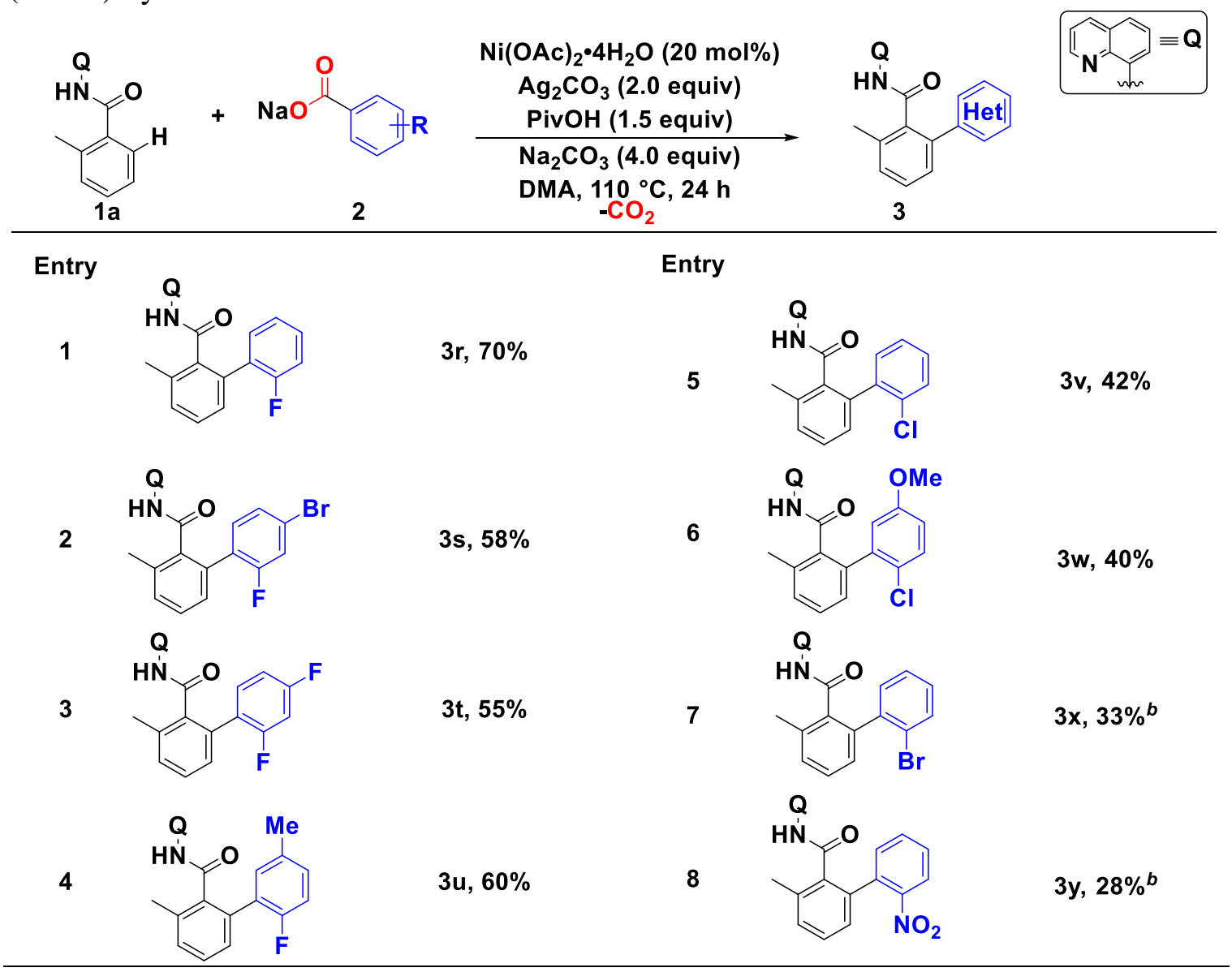

${ }^{a}$ Isolated yields. Reaction conditions: Benzamide $1 \mathbf{a}(0.2 \mathrm{mmol})$, benzoate $2(0.6 \mathrm{mmol})$, $\mathrm{Ni}(\mathrm{OAc})_{2} \bullet 4 \mathrm{H}_{2} \mathrm{O}$ (20 mol \%), $\mathrm{Ag}_{2} \mathrm{CO}_{3}$ (2.0 equiv), $\mathrm{Na}_{2} \mathrm{CO}_{3}$ (4.0 equiv), and $\mathrm{PivOH}$ (1.5 equiv) in DMA $(2 \mathrm{~mL})$ for $24 \mathrm{~h}$ at $110{ }^{\circ} \mathrm{C}$ under a $\mathrm{N}_{2}$ atmosphere. ${ }^{b 1} \mathrm{H}$ NMR yield with 1,3,5-trimethoxybenzene as an internal standard.

Lastly, we explored the scope of the substituted benzamides (Table 2.5). Benzamides with different substituents were explored first. When the unsubstituted benzamide is submitted to the standard reaction conditions, both mono- and diarylation products were formed. Benzamides with meta-substituents (3aa - 3ad) showed regioselective arylation at the less-hindered ortho- $\mathrm{C}-\mathrm{H}$ bond. This new catalyst system again tolerates aryl 
halides (3ac and 3ad) as seen in Table 2.4. Other benzamides such as naphthyl (3ae) and $N$-methylpyrrole (3af) also undergo arylation in good yields.

Table 2.5. Benzamide scope of the nickel-catalyzed oxidative decarboxylative (hetero)arylation ${ }^{a}$

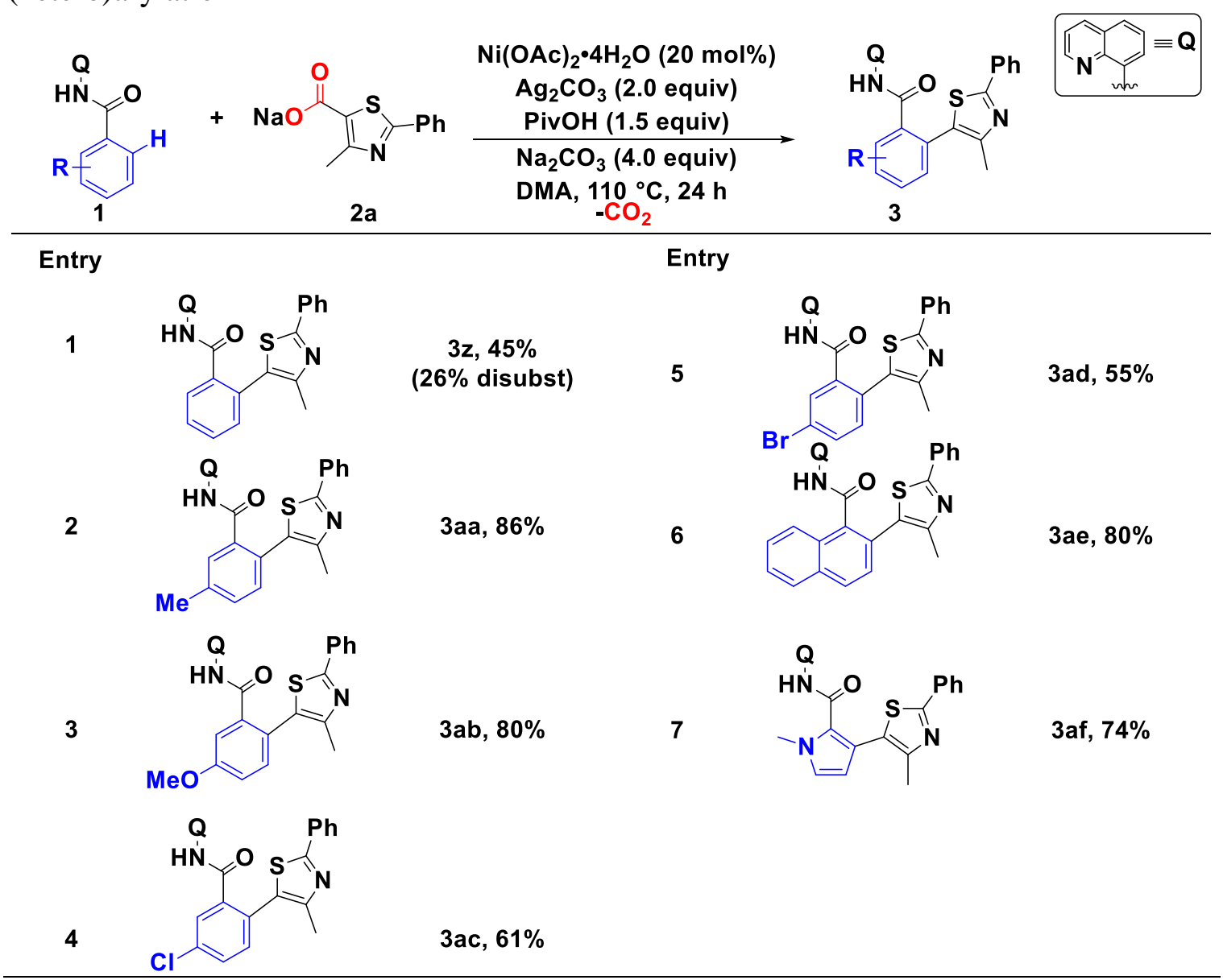

${ }^{a}$ Isolated yields. Reaction conditions: Benzamide $1(0.2 \mathrm{mmol})$, heteroaromatic carboxylate 2a $(0.6 \mathrm{mmol}), \mathrm{Ni}(\mathrm{OAc})_{2} \bullet 4 \mathrm{H}_{2} \mathrm{O}(20 \mathrm{~mol} \%), \mathrm{Ag}_{2} \mathrm{CO}_{3}$ (2.0 equiv), $\mathrm{Na}_{2} \mathrm{CO}_{3}$ (4.0 equiv), and $\mathrm{PivOH}$ (1.5 equiv) in DMA ( $2 \mathrm{~mL}$ ) for $24 \mathrm{~h}$ at $110^{\circ} \mathrm{C}$ under a $\mathrm{N}_{2}$ atmosphere.

\subsubsection{Preliminary Mechanistic Experiments of the Nickel-Catalyzed Oxidative Decarboxylative (Hetero)Arylation}

To gain insight into the reaction pathway of this new reaction, we conducted a series of experiments. As mentioned earlier in the optimization of the reaction conditions, when there is no nickel or silver present no product is formed (Table 2.6). When the standard reaction was conducted only in the presence of silver no product 3a was formed, however, $85 \%$ of the protodecarboxylated 4-methyl-2-phenyl-1,3-thiazole was recovered 
(Table 2.6 entry 2). In contrast, when the experiment is conducted in the presence of only nickel there is quantitative recovery of the heteroaromatic carboxylic acid (2a" $\mathbf{a}^{\prime \prime}$, entry 3$)$. These data show that silver could be responsible for decarboxylation, while nickel is needed for the $\mathrm{C}-\mathrm{C}$ bond formation.

Table 2.6. Control experiments of the nickel-catalyzed oxidative decarboxylative (hetero)arylation ${ }^{a}$

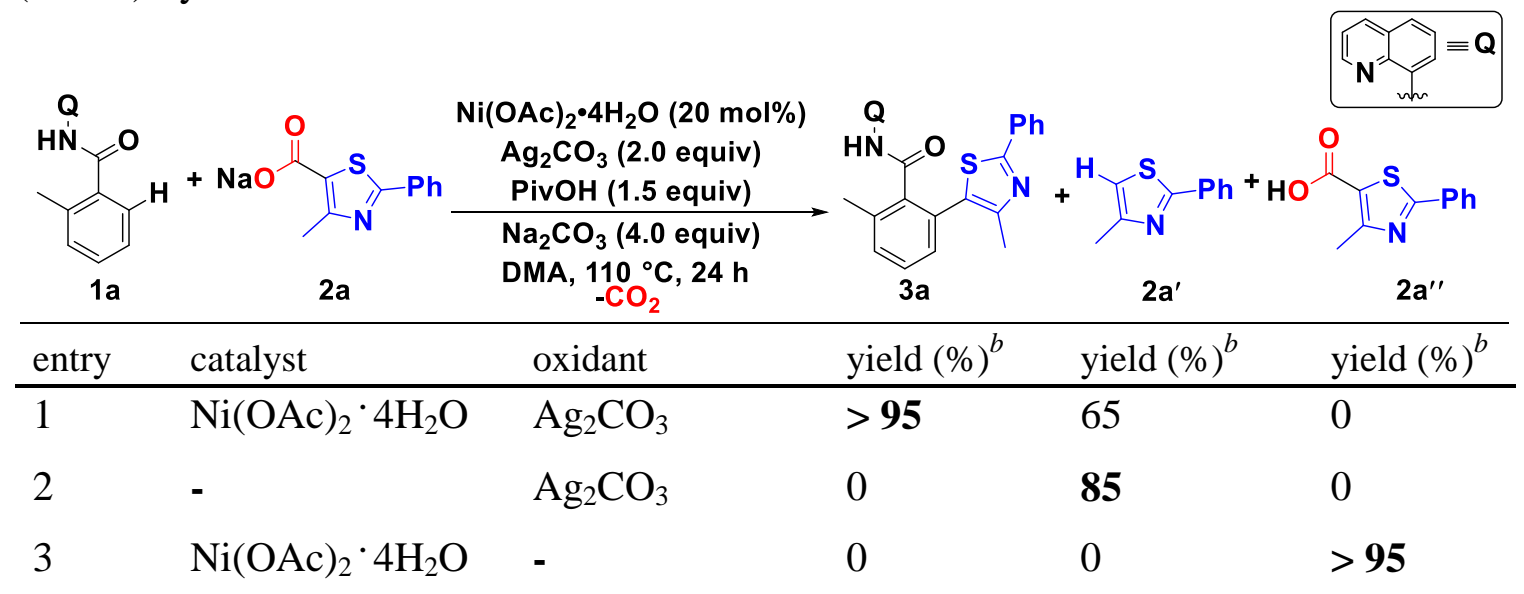

${ }^{a}$ Reaction conditions: Benzamide 1a $(0.2 \mathrm{mmol})$, heteroaromatic carboxylate 2a $(0.6$ mmol), $\mathrm{Ni}(\mathrm{OAc})_{2} \bullet 4 \mathrm{H}_{2} \mathrm{O}$ (20 mol \%), $\mathrm{Ag}_{2} \mathrm{CO}_{3}$ (2.0 equiv), $\mathrm{Na}_{2} \mathrm{CO}_{3}$ (4.0 equiv), and PivOH (1.5 equiv) in DMA ( $2 \mathrm{~mL})$ for $24 \mathrm{~h}$ at $110{ }^{\circ} \mathrm{C}$ under a $\mathrm{N}_{2}$ atmosphere. ${ }^{b 1} \mathrm{H}$ NMR yield with 1,3,5-trimethoxybenzene as an internal standard.

As previously mentioned in Chapter 1, it is known that silver salts can promote the decarboxylation of benzoic acids to generate silver-aryl or radical intermediates. ${ }^{16} \mathrm{We}$ first probed our reaction conditions with radical trapping reagents such as TEMPO and DHA (Table 2.7). In the presence of 1 equiv of either radical scavenger, there were no trappable radical intermediates and no decrease in the yield of the desired product 3a. These data suggest there could be a silver-aryl intermediate that plays a key role in this reaction. 
Table 2.7. Radical scavengers with the nickel-catalyzed oxidative decarboxylative (hetero)arylation ${ }^{a}$

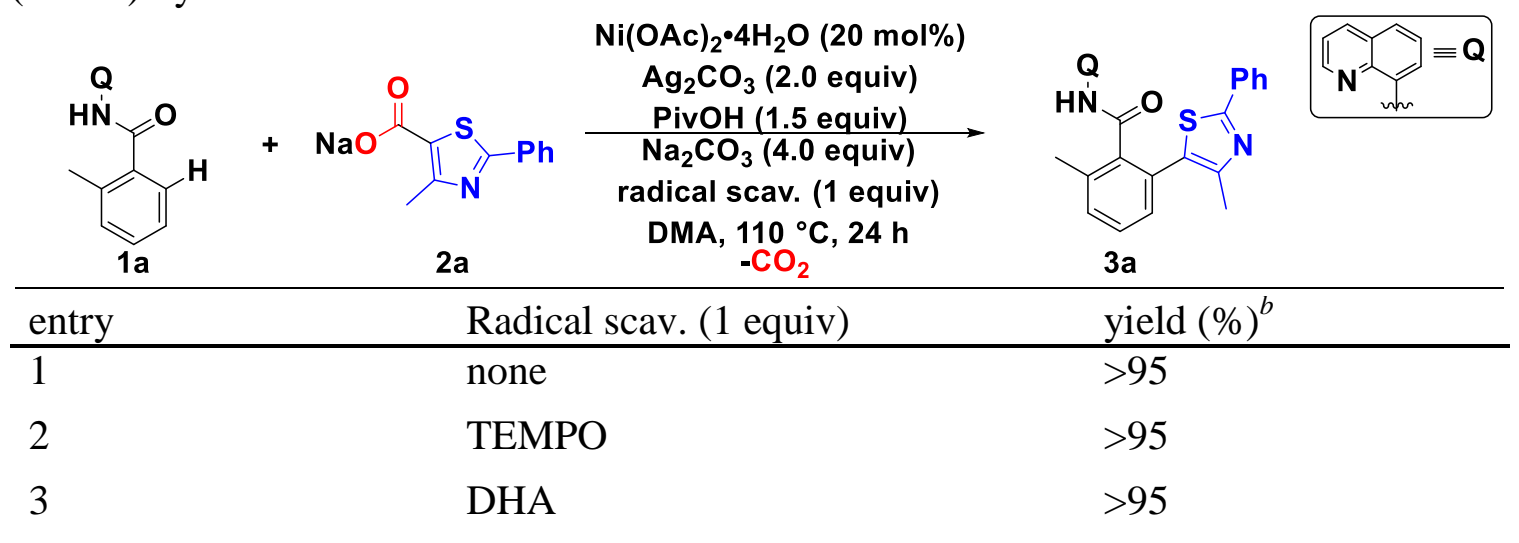

${ }^{a}$ Reaction conditions: Benzamide 1a $(0.2 \mathrm{mmol})$, heteroaromatic carboxylate 2a $(0.6$ mmol), $\mathrm{Ni}(\mathrm{OAc})_{2} \bullet 4 \mathrm{H}_{2} \mathrm{O}$ (20 mol \%), $\mathrm{Ag}_{2} \mathrm{CO}_{3}$ (2.0 equiv), $\mathrm{Na}_{2} \mathrm{CO}_{3}$ (4.0 equiv), radical scavenger (1.0 equiv) and PivOH (1.5 equiv) in DMA (2 mL) for $24 \mathrm{~h}$ at $110^{\circ} \mathrm{C}$ under a $\mathrm{N}_{2}$ atmosphere. ${ }^{b 1} \mathrm{H}$ NMR yield with 1,3,5-trimethoxybenzene as an internal standard.

Next, we explored the $\mathrm{C}-\mathrm{H}$ activation step of our new reaction method. The kinetic isotope effect obtained from the intermolecular competition (Scheme 15a) experiment reveled $k_{\mathrm{H}} / k_{\mathrm{D}}=4.3$. The deuterium-exchange of $\mathbf{1 a -} \boldsymbol{d}_{\mathbf{7}}$ under our standard reaction conditions showed no proton incorporation of the ortho-C-D bond in the recovered starting material (Scheme 15b) by ${ }^{1} \mathrm{H}$ NMR spectroscopy. These data suggest that the $\mathrm{C}-\mathrm{H}$ activation step is irreversible and consistent with a primary isotope effect. ${ }^{44}$ If this is the case, we might be able to isolate a stable nickel metallacycle; this will be discussed later in Chapter 4. 
Scheme 15. Kinetic isotope effect of the nickel-catalyzed oxidative decarboxylative (hetero)arylation. ${ }^{a}$

(a) Intermolecular KIE Experiment ${ }^{\mathrm{a}, \mathrm{c}}$
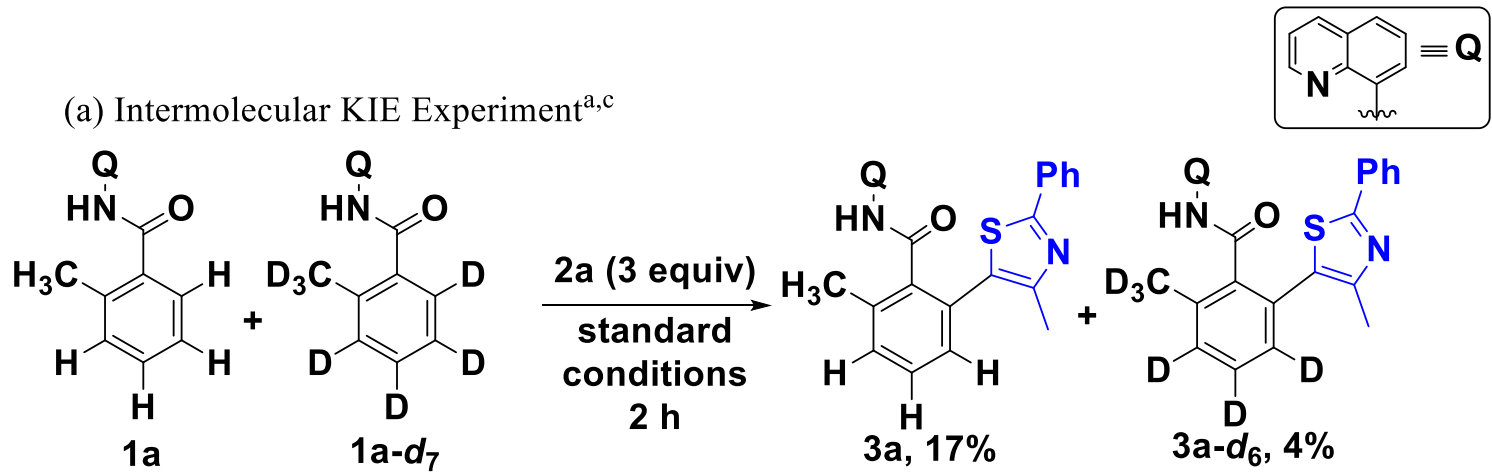

(b) Deuterium-Exchange Experiment ${ }^{\mathrm{b}, \mathrm{c}}$

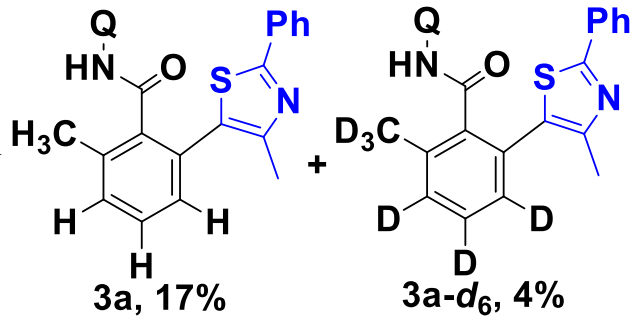

Intermolecular KIE = 4.3<smiles>[2H]c1c([2H])c([2H])c(C(=O)NO)c(C(=O)O)c1[2H]</smiles>

$1 a-d_{7}$

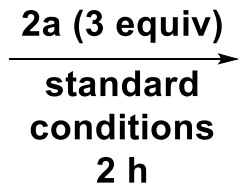

$2 \mathrm{~h}$<smiles>[2H]c1c([2H])c(C(=O)NO)c(-c2sc(-c3ccccc3)nc2C)c(C([2H])([2H])[2H])c1[2H]</smiles>

$3 a-d_{6}$ $8 \%$ yield<smiles>[2H]c1c([2H])c([2H])c(C(=O)NO)c(C(=O)O)c1[2H]</smiles>

1a-d 7 $90 \%$ yield, $0 \% \mathrm{H}$

${ }^{a}$ Reaction conditions: Benzamide 1a $(0.1 \mathrm{mmol})$, 1a- $\boldsymbol{d}_{\mathbf{7}}(0.1 \mathrm{mmol})$, heteroaromatic carboxylate $2 \mathbf{a}(0.6 \mathrm{mmol}), \mathrm{Ni}(\mathrm{OAc})_{2} \bullet 4 \mathrm{H}_{2} \mathrm{O}(20 \mathrm{~mol} \%), \mathrm{Ag}_{2} \mathrm{CO}_{3}$ (2.0 equiv), $\mathrm{Na}_{2} \mathrm{CO}_{3}$ (4.0 equiv), and $\mathrm{PivOH}$ (1.5 equiv) in DMA $(2 \mathrm{~mL})$ for $2 \mathrm{~h}$ at $110{ }^{\circ} \mathrm{C}$ under a $\mathrm{N}_{2}$ atmosphere. ${ }^{b}$ Benzamide $\mathbf{1 a}-\boldsymbol{d}_{\mathbf{7}}(0.2 \mathrm{mmol})$, Heteroaromatic carboxylate $\mathbf{2 a}(0.6 \mathrm{mmol})$, $\mathrm{Ni}(\mathrm{OAc})_{2} \bullet 4 \mathrm{H}_{2} \mathrm{O}$ (20 mol \%), $\mathrm{Ag}_{2} \mathrm{CO}_{3}$ (2.0 equiv), $\mathrm{Na}_{2} \mathrm{CO}_{3}$ (4.0 equiv), and $\mathrm{PivOH}$ (1.5 equiv) in DMA ( $2 \mathrm{~mL}$ ) for $2 \mathrm{~h}$ at $110{ }^{\circ} \mathrm{C}$ under a $\mathrm{N}_{2}$ atmosphere. ${ }^{c 1} \mathrm{H}$ NMR yield with 1,3,5-trimethoxybenzene as an internal standard.

\subsubsection{Proposed Reaction Mechanism of the Nickel-Catalyzed Oxidative}

\section{Decarboxylative (Hetero)Arylation Reaction}

From the results of the control experiments and kinetic isotope effect experiments mentioned above, we have proposed a mechanism for our new catalytic reaction (Scheme 16). The pre-catalyst enters the reaction pathway and becomes the active catalyst followed by ligand exchange with the amide $\mathbf{A}$ and then forming the nickel(II) metallacycle B. During this time, silver(I) carboxylate is formed and undergoes decarboxylation to form a silver(I)-aryl. The silver(I)-aryl undergoes transmetallation 
with nickel complex $\mathbf{B}$ along with concomitant oxidation to form the nickel(III) metallacycle $\mathbf{C}$. Reductive elimination from intermediate $\mathbf{C}$ generates the new $\mathrm{C}-\mathrm{C}$ bond to form $\mathbf{D}$. Intermediate $\mathbf{D}$ undergoes protonation and reoxidation of nickel(I) to close the catalytic cycle. We believe the remarkable efficiency of this new catalyst system is due to the cooperation of both nickel and silver together.

Scheme 16. Proposed reaction mechanism of the nickel-catalyzed oxidative decarboxylative (hetero)arylation.

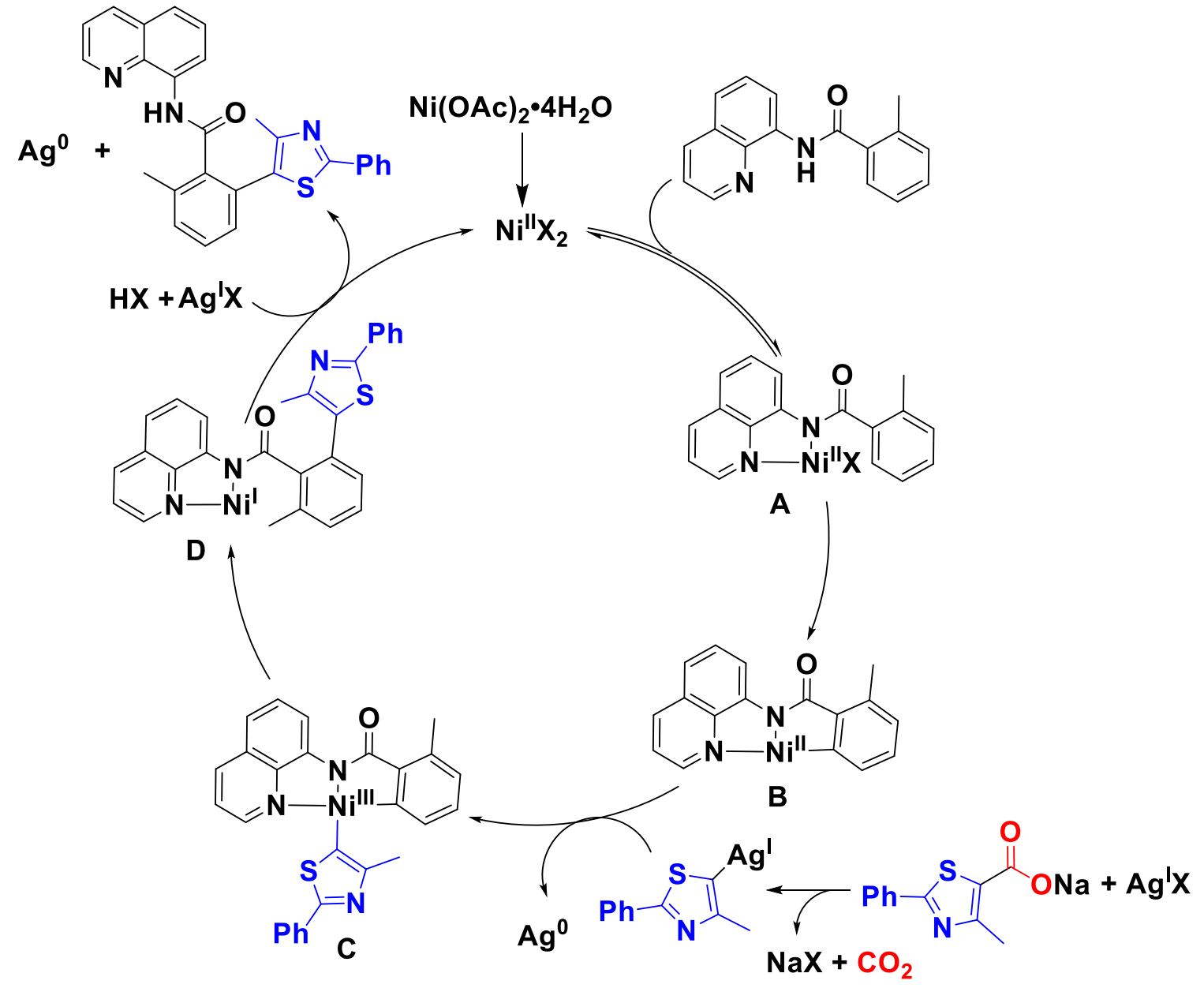




\subsection{Conclusion}

In summary, we have developed the first nickel-catalyzed oxidative decarboxylative (hetero)arylation of unactivated $\mathrm{C}-\mathrm{H}$ bonds. This method employs the use of $\mathrm{Ni}(\mathrm{OAc})_{2} \bullet 4 \mathrm{H}_{2} \mathrm{O}$ as the catalyst, $\mathrm{Ag}_{2} \mathrm{CO}_{3}$ as the oxidant, $\mathrm{Na}_{2} \mathrm{CO}_{3}$ as the base and PivOH as an additive to enable the efficient coupling of a broad scope of heteroaromatic carboxylates and ortho-substituted benzoates. The data from our control experiments show there could be cooperation between both nickel and silver. Silver could be responsible for decarboxylation and generation of a silver(I)-aryl, while nickel is needed for formation of the $\mathrm{C}-\mathrm{C}$ bond.

\subsection{Experimental}

General Information. All manipulations were performed using standard Schlenk or glovebox techniques under a nitrogen atmosphere. All solvents (including dry DMA) were bought from Alfa-Aesar, Fisher, and Cambridge Isotope and used as received. All other reagents were purchased from Maybridge, Oakwood, Acros, Alfa-Aesar, Astatech, Strem and CDN Isotopes and used without further purification. All NMR spectra were recorded at ambient temperature using Agilent $400 \mathrm{MHz}\left({ }^{1} \mathrm{H}, 400 \mathrm{MHz} ;{ }^{13} \mathrm{C}\left\{{ }^{1} \mathrm{H}\right\}, 100\right.$ $\mathrm{MHz} ;{ }^{19} \mathrm{~F}, 376 \mathrm{MHz}$ ) spectrometer. Chemical shifts are referenced to the residual solvent signals $\left(\mathrm{CDCl}_{3},{ }^{1} \mathrm{H}: 7.26 \mathrm{ppm},{ }^{13} \mathrm{C}: 77.2 \mathrm{ppm}\right)$ and (DMSO- $d_{6},{ }^{1} \mathrm{H}: 2.50 \mathrm{ppm},{ }^{13} \mathrm{C}: 39.5$ ppm). ${ }^{45}$ High resolution mass spectra were obtained on a Thermo Finnigan Linear Trapping Quadrupole mass spectrometer. IR spectra were recorded on a PerkinElmer (Spectrum 100) FT-IR spectrometer. Column chromatography was performed using Silicycle Silia Flash P60 silica gel.

\section{General Procedure for the Optimization of the Oxidative Decarboxylative Arylation}

Reaction. An oven-dried $50 \mathrm{~mL}$ Schlenk tube with a stirring bar was charged with 4methy-2-phenyl-1,3-thiazole-5-carboxylic acid $\mathbf{2 a}$ (131 mg, $0.600 \mathrm{mmol}$ ), sodium carbonate $(63 \mathrm{mg}, 0.60 \mathrm{mmol})$, and dry DMA $(1 \mathrm{~mL})$. The reaction vessel was placed in a pre-heated oil bath and stirred for $0.5 \mathrm{~h}$ at $130{ }^{\circ} \mathrm{C}$. The solvent was removed under reduced pressure until dry and the carboxylate salt was used without further purification. 2-methyl-N-(quinolin-8-yl) benzamide $1 \mathbf{a}(52.4 \mathrm{mg}, 0.200 \mathrm{mmol}), \mathrm{Ni}(\mathrm{OAc})_{2} \bullet 4 \mathrm{H}_{2} \mathrm{O}(9.9$ 
$\mathrm{mg}, 0.040 \mathrm{mmol}), \mathrm{Ag}_{2} \mathrm{CO}_{3}(110 \mathrm{mg}, 0.400 \mathrm{mmol}), \mathrm{Na}_{2} \mathrm{CO}_{3}(83 \mathrm{mg}, 0.80 \mathrm{mmol})$, and $\mathrm{PivOH}(30.6 \mathrm{mg}, 0.300 \mathrm{mmol}$ ) were added and the tube was evacuated and backfilled with nitrogen three times after which DMA $(2 \mathrm{~mL})$ was added via syringe. The reaction mixture was stirred at $110{ }^{\circ} \mathrm{C}$ for $24 \mathrm{~h}$. Upon completion, the reaction tube was cooled to room temperature. The solution was diluted with ethyl acetate $(25 \mathrm{~mL})$ and poured into a $250 \mathrm{~mL}$ separatory funnel. To the solution, water (25 mL), $\mathrm{Na}_{2}$ EDTA (500 mg), aqueous $\mathrm{HCl}(1 \mathrm{~N}, 10 \mathrm{~mL})$ were added and extracted with ethyl acetate $(2 \times 30 \mathrm{~mL})$. The combined organic layers were washed with water $(100 \mathrm{~mL})$ and brine $(25 \mathrm{~mL})$, dried over $\mathrm{Na}_{2} \mathrm{SO}_{4}$, filtered and concentrated under vacuum. Then 1,3,5-trimethoxybenzene (5.00 mg) was added to the residue and the crude mixture was dissolved in $\mathrm{CDCl}_{3}$ for ${ }^{1} \mathrm{H}$ analysis.

\section{Optimization of the Pre-catalyst ${ }^{a}$}

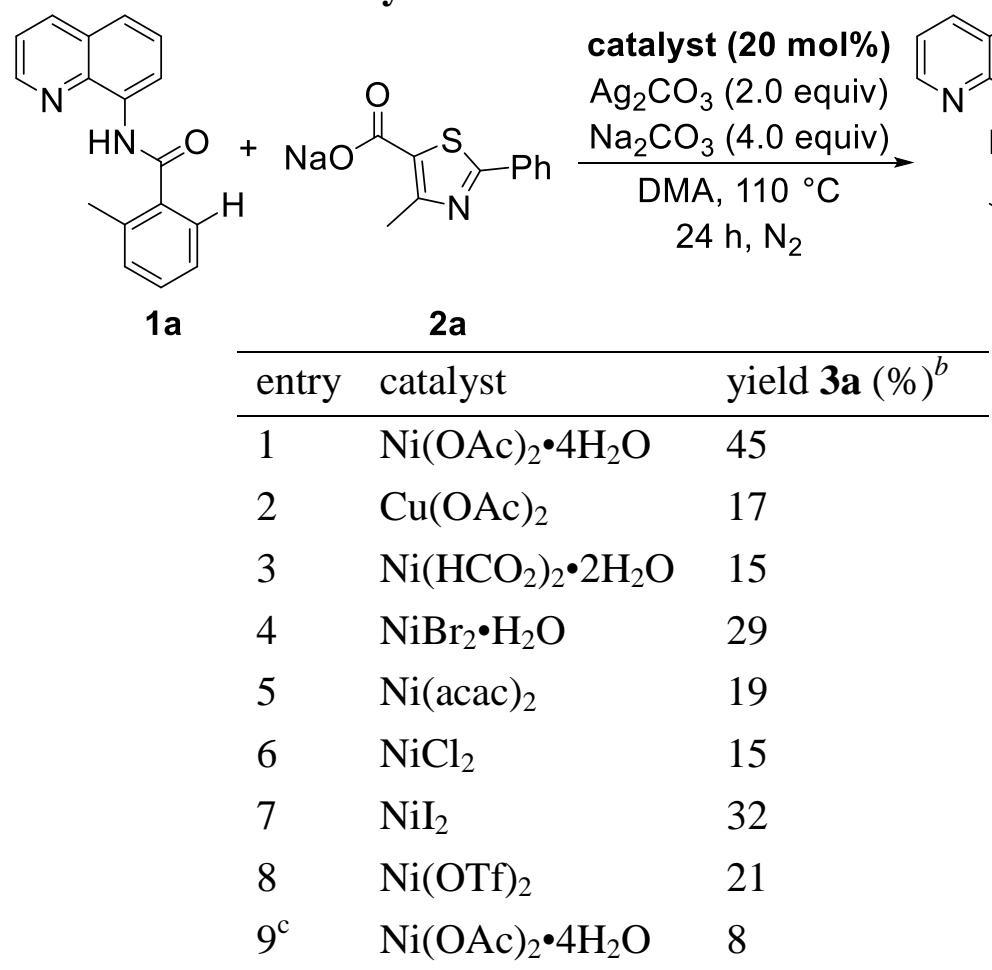

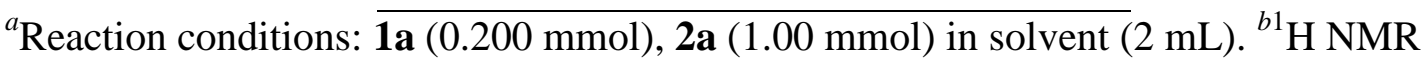
yield with 1,3,5-trimethoxybenzene as an internal standard. ${ }^{c} 10 \mathrm{~mol} \%$ 


\section{Optimization of the Oxidant ${ }^{a}$}

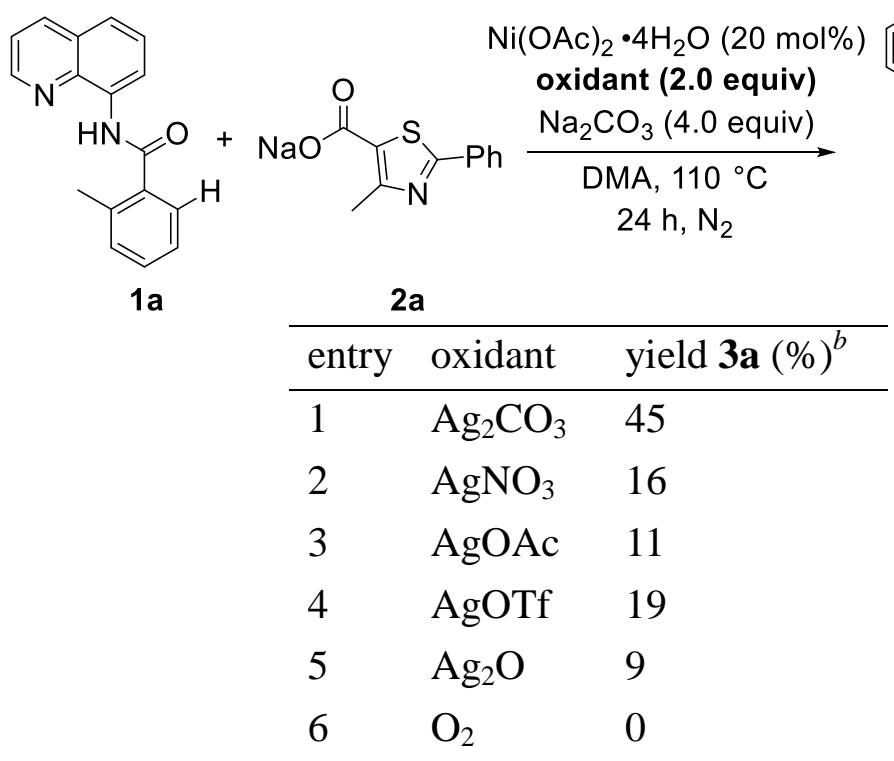

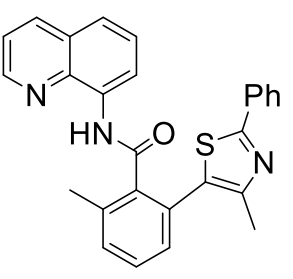

$3 \mathbf{a}$

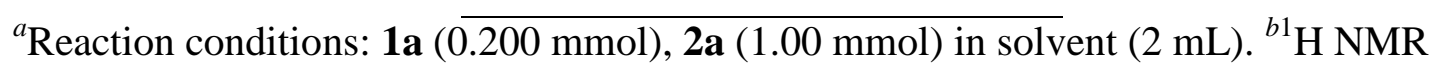
yield with 1,3,5-trimethoxybenzene as an internal standard.

\section{Optimization of the Solvent ${ }^{a}$}

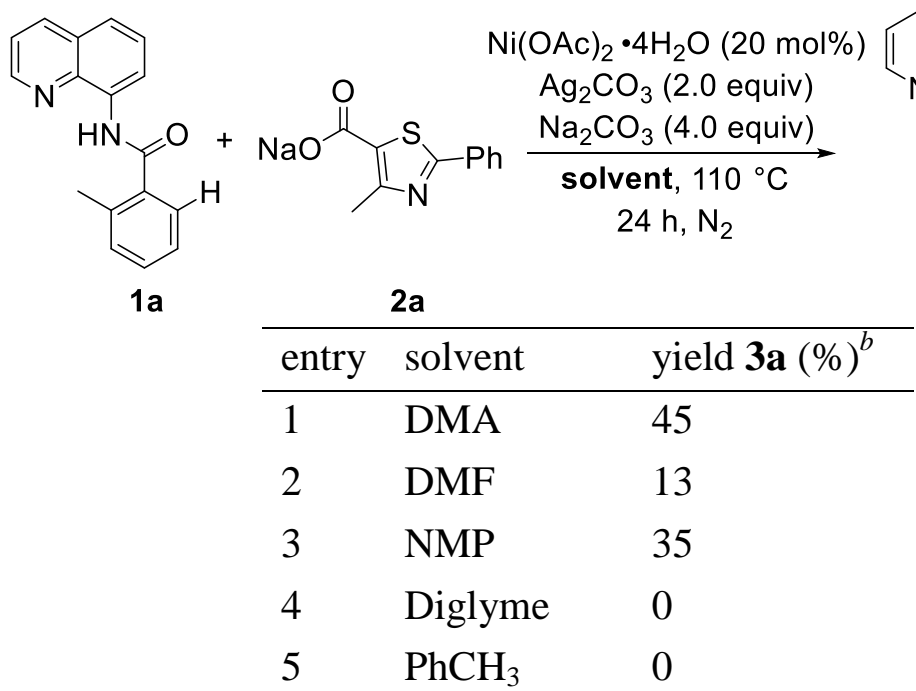

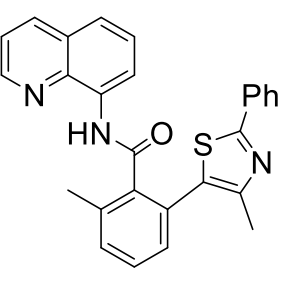

$3 \mathbf{a}$

\begin{tabular}{lll}
\hline entry & solvent & yield 3a $(\%)^{b}$ \\
\hline 1 & DMA & 45 \\
2 & DMF & 13 \\
3 & NMP & 35 \\
4 & Diglyme & 0 \\
5 & $\mathrm{PhCH}_{3}$ & 0
\end{tabular}

${ }^{a}$ Reaction conditions: 1a $\overline{(0.200 \mathrm{mmol}), \mathbf{2 a}(1.00 \mathrm{mmol}) \text { in solvent }}(2 \mathrm{~mL}) .{ }^{b 1} \mathrm{H} \mathrm{NMR}$ yield with 1,3,5-trimethoxybenzene as an internal standard. 


\section{Optimization of the Base ${ }^{a}$}

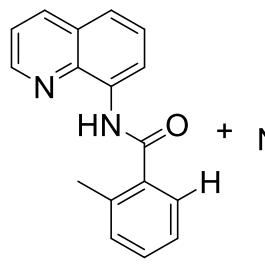

1a

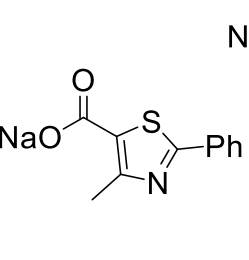

$2 \mathbf{a}$

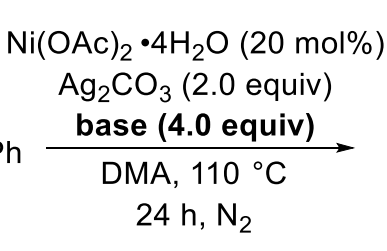

$24 \mathrm{~h}, \mathrm{~N}_{2}$

\begin{tabular}{lll}
\multicolumn{2}{c}{ 2a } & \\
\hline entry & {$[$ base] } & yield 3a $(\%)^{b}$ \\
\hline 1 & $\mathrm{Na}_{2} \mathrm{CO}_{3}$ & 45 \\
2 & $\mathrm{Li}_{2} \mathrm{CO}_{3}$ & 20 \\
3 & $\mathrm{~K}_{2} \mathrm{CO}_{3}$ & 0 \\
4 & $\mathrm{NaOAc}_{2}$ & 25 \\
5 & pyridine & 15
\end{tabular}

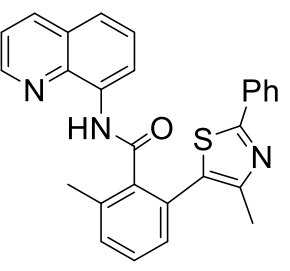

$3 a$

${ }^{a}$ Reaction conditions: 1a $\left(\overline{0.200 \mathrm{mmol}), \mathbf{2 a}(1.00 \mathrm{mmol}) \text { in solvent }}(2 \mathrm{~mL}) .{ }^{b 1} \mathrm{H} \mathrm{NMR}\right.$ yield with 1,3,5-trimethoxybenzene as an internal standard.

\section{Optimization of the Additives ${ }^{a}$}

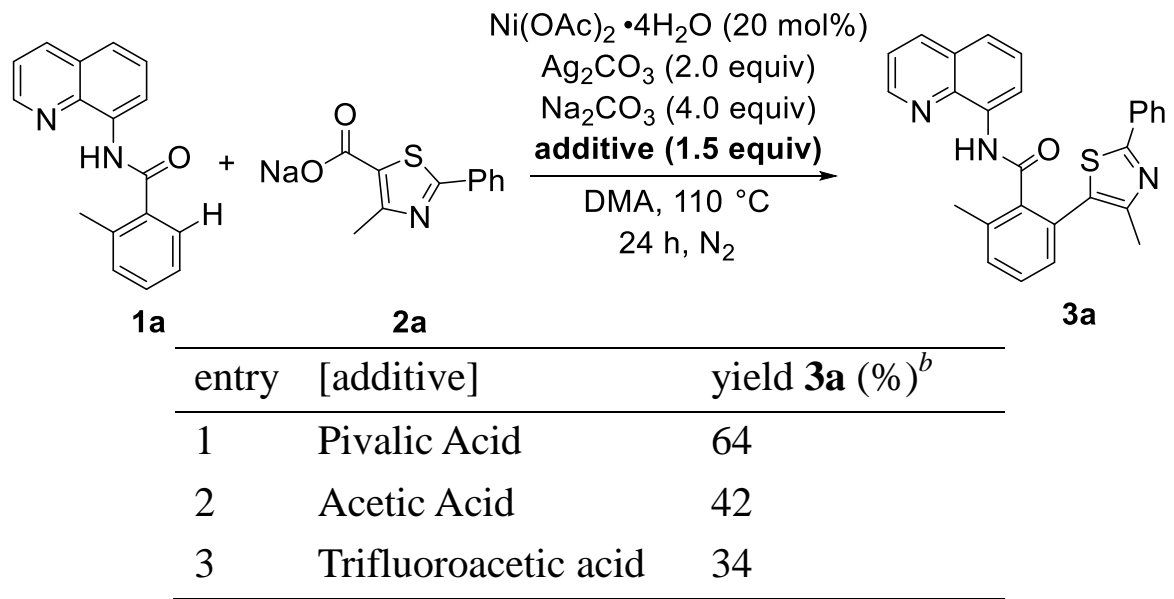

${ }^{a}$ Reaction conditions: 1a $(0.200 \mathrm{mmol}), \mathbf{2 a}(1.00 \mathrm{mmol})$ in solvent $(2 \mathrm{~mL}) .{ }^{b 1} \mathrm{H} \mathrm{NMR}$ yield with 1,3,5-trimethoxybenzene as an internal standard.

\section{General Procedure for the Ni-Catalyzed Oxidative Decarboxylative Arylation.}

Procedure A (Generation of Sodium Salts at $130{ }^{\circ} \mathbf{C}$ ): An oven-dried $50 \mathrm{~mL}$ Schlenk tube with a stirring bar was charged with carboxylic acid 2 (0.600 mmol), $\mathrm{Na}_{2} \mathrm{CO}_{3}(63$ $\mathrm{mg}, 0.60 \mathrm{mmol})$, and dry DMA $(1 \mathrm{~mL})$. The reaction vessel was placed in a pre-heated oil bath and stirred for $0.5 \mathrm{~h}$ at $130{ }^{\circ} \mathrm{C}$. The solvent was removed under reduced pressure until dry and the carboxylate salt was used without further purification. $N$-(quinolin-8-yl) 
benzamide $1(0.200 \mathrm{mmol}), \mathrm{Ni}(\mathrm{OAc})_{2} \bullet 4 \mathrm{H}_{2} \mathrm{O}(9.9 \mathrm{mg}, 0.040 \mathrm{mmol}), \mathrm{Ag}_{2} \mathrm{CO}_{3}(110 \mathrm{mg}$, $0.400 \mathrm{mmol}), \mathrm{Na}_{2} \mathrm{CO}_{3}(83 \mathrm{mg}, 0.80 \mathrm{mmol})$, and PivOH (30.6 mg, $\left.0.300 \mathrm{mmol}\right)$ were added and the tube was evacuated and backfilled with nitrogen three times after which DMA (2 mL) was added via syringe. The reaction mixture was stirred at $110{ }^{\circ} \mathrm{C}$ for $24 \mathrm{~h}$. Upon completion, the reaction tube was cooled to room temperature. The solution was diluted with ethyl acetate $(25 \mathrm{~mL})$ and poured into a $250 \mathrm{~mL}$ separatory funnel. To the solution, water $(25 \mathrm{~mL}), \mathrm{Na}_{2}$ EDTA $(500 \mathrm{mg})$, aqueous $\mathrm{HCl}(1 \mathrm{~N}, 10 \mathrm{~mL})$ were added and the mixture was extracted with ethyl acetate $(2 \times 30 \mathrm{~mL})$. The combined organic layers were washed with water $(100 \mathrm{~mL})$ and brine $(25 \mathrm{~mL})$, dried over $\mathrm{Na}_{2} \mathrm{SO}_{4}$, filtered and concentrated under vacuum. The residue was purified by silica gel column chromatography (gradient elution, hexanes : ethyl acetate $(19: 1, \mathrm{v} / \mathrm{v})$ to $(1: 1, \mathrm{v} / \mathrm{v}))$, to yield the corresponding product.

Procedure B (Generation of Sodium Salts at $\left.110{ }^{\circ} \mathbf{C}\right)$ : An oven-dried $50 \mathrm{~mL}$ Schlenk tube with a stirring bar was charged with carboxylic acid 2 (0.600 mmol), $\mathrm{Na}_{2} \mathrm{CO}_{3}(63$ $\mathrm{mg}, 0.60 \mathrm{mmol})$, and dry DMA $(1 \mathrm{~mL})$. The reaction vessel was placed in a pre-heated oil bath and stirred for $0.5 \mathrm{~h}$ at $110{ }^{\circ} \mathrm{C}$. The solvent was removed under reduced pressure until dry and the carboxylate salt was used without further purification. $N$-(quinolin-8-yl) benzamide $1(0.200 \mathrm{mmol}), \mathrm{Ni}(\mathrm{OAc})_{2} \bullet 4 \mathrm{H}_{2} \mathrm{O}(9.9 \mathrm{mg}, 0.040 \mathrm{mmol}), \mathrm{Ag}_{2} \mathrm{CO}_{3}(110 \mathrm{mg}$, $0.400 \mathrm{mmol}), \mathrm{Na}_{2} \mathrm{CO}_{3}(83 \mathrm{mg}, 0.80 \mathrm{mmol})$, and $\mathrm{PivOH}(30.6 \mathrm{mg}, 0.300 \mathrm{mmol})$ were added and the tube was evacuated and backfilled with nitrogen three times after which DMA (2 mL) was added via syringe. The reaction mixture was stirred at $110{ }^{\circ} \mathrm{C}$ for $24 \mathrm{~h}$. Upon completion, the reaction tube was cooled to room temperature. The solution was diluted with ethyl acetate $(25 \mathrm{~mL})$ and poured into a $250 \mathrm{~mL}$ separatory funnel. To the solution, water (25 mL), $\mathrm{Na}_{2}$ EDTA $(500 \mathrm{mg})$, aqueous $\mathrm{HCl}(1 \mathrm{~N}, 10 \mathrm{~mL})$ were added and the mixture was extracted with ethyl acetate $(2 \times 30 \mathrm{~mL})$. The combined organic layers were washed with water $(100 \mathrm{~mL})$ and brine $(25 \mathrm{~mL})$, dried $\mathrm{Na}_{2} \mathrm{SO}_{4}$, filtered and concentrated in vacuo. The residue was purified by silica gel column chromatography (gradient elution, hexanes:ethyl acetate $(19: 1, \mathrm{v} / \mathrm{v})$ to $(1: 1, \mathrm{v} / \mathrm{v}))$, yielding the corresponding product. 


\section{Reactions with Radical Scavengers.}

An oven-dried $50 \mathrm{~mL}$ Schlenk tube with a stirring bar was charged with 4-methy-2phenyl-1,3-thiazole-5-carboxylic acid 2a (131 mg, $0.600 \mathrm{mmol}$ ), sodium carbonate (63 $\mathrm{mg}, 0.60 \mathrm{mmol})$, and dry DMA $(1 \mathrm{~mL})$. The reaction vessel was placed in a pre-heated oil bath and stirred for $0.5 \mathrm{~h}$ at $130{ }^{\circ} \mathrm{C}$. The solvent was removed under reduced pressure until dry and the resulting carboxylate salt was used without further purification. 2methyl-N-(quinolin-8-yl) benzamide 1 a $(52.4 \mathrm{mg}, 0.200 \mathrm{mmol}), \mathrm{Ni}(\mathrm{OAc})_{2} \bullet 4 \mathrm{H}_{2} \mathrm{O}(9.9$ $\mathrm{mg}, 0.040 \mathrm{mmol}), \mathrm{Ag}_{2} \mathrm{CO}_{3}(110 \mathrm{mg}, 0.400 \mathrm{mmol}), \mathrm{Na}_{2} \mathrm{CO}_{3}(83 \mathrm{mg}, 0.80 \mathrm{mmol})$, TEMPO (31.2 mg, $0.200 \mathrm{mmol})$ or DHA (36.0, $0.200 \mathrm{mmol})$, and PivOH (30.6 mg, $0.300 \mathrm{mmol}$ ) were added and the tube was evacuated and backfilled with nitrogen three times after which DMA $(2 \mathrm{~mL})$ was added via syringe. The reaction mixture was stirred at $110{ }^{\circ} \mathrm{C}$ for $24 \mathrm{~h}$. Upon completion, the reaction tube was cooled to room temperature. The solution was diluted with ethyl acetate $(25 \mathrm{~mL})$ and poured into a $250 \mathrm{~mL}$ separatory funnel. To the solution, water $(25 \mathrm{~mL}), \mathrm{Na}_{2}$ EDTA $(500 \mathrm{mg})$, aqueous $\mathrm{HCl}(1 \mathrm{~N}, 10 \mathrm{~mL})$ were added and extracted with ethyl acetate $(2 \times 30 \mathrm{~mL})$. The combined organic layers were washed with water $(100 \mathrm{~mL})$ and brine $(25 \mathrm{~mL})$, dried over $\mathrm{Na}_{2} \mathrm{SO}_{4}$, filtered and concentrated under vacuum. Then 1,3,5-trimethoxybenzene $(5.00 \mathrm{mg})$ was added to the residue and the crude mixture was dissolved in $\mathrm{CDCl}_{3}$ for ${ }^{1} \mathrm{H}$ analysis.

Procedure for the Intermolecular Competition Experiment. An oven-dried $50 \mathrm{~mL}$ Schlenk tube with a stirring bar was charged with carboxylic acid $2(0.600 \mathrm{mmol})$, sodium carbonate $(63 \mathrm{mg}, 0.60 \mathrm{mmol})$, and dry DMA $(1 \mathrm{~mL})$. The reaction vessel was placed in a pre-heated oil bath and stirred for $0.5 \mathrm{~h}$ at $130{ }^{\circ} \mathrm{C}$. Solvent was removed under

reduced pressure until dry and the resulting carboxylate salt was used without further purification. 1a $(26.2 \mathrm{mg}, 0.100 \mathrm{mmol}), \mathbf{1 a}-\boldsymbol{d}_{7}(26.9 \mathrm{mg}, 0.100 \mathrm{mmol}), \mathrm{Ni}(\mathrm{OAc})_{2} \bullet 4 \mathrm{H}_{2} \mathrm{O}$ (9.9 mg, $0.040 \mathrm{mmol}$ ), $\mathrm{Ag}_{2} \mathrm{CO}_{3}\left(110 \mathrm{mg}, 0.400 \mathrm{mmol}\right.$ ), $\mathrm{Na}_{2} \mathrm{CO}_{3}(83 \mathrm{mg}, 0.80 \mathrm{mmol}$ ), PivOH (30.6 mg, $0.300 \mathrm{mmol}$ ) were added, the tube was evacuated and backfilled with nitrogen three times after which DMA $(2 \mathrm{~mL})$ was added via syringe. The reaction mixture was stirred at $110{ }^{\circ} \mathrm{C}$ for $2 \mathrm{~h}$. Upon completion, the reaction tube was cooled to 
room temperature. The solution was diluted with ethyl acetate $(25 \mathrm{~mL})$ and poured into a $250 \mathrm{~mL}$ separatory funnel. To the solution, water (25 mL), $\mathrm{Na}_{2}$ EDTA (500 mg), aqueous $\mathrm{HCl}(1 \mathrm{~N}, 10 \mathrm{~mL})$ were added and extracted with ethyl acetate $(2 \times 30 \mathrm{~mL})$. The combined organic layers were washed with water $(100 \mathrm{~mL})$ and brine $(25 \mathrm{~mL})$, dried $\mathrm{Na}_{2} \mathrm{SO}_{4}$, filtered and concentrated in vacuo. The residue was purified by silica gel column chromatography (gradient elution, hexanes:ethyl acetate $(19: 1, \mathrm{v} / \mathrm{v})$ to $(4: 1, \mathrm{v} / \mathrm{v}))$, yielding the corresponding product of $\mathbf{3 a}$ and $\mathbf{3 a}-\boldsymbol{d}_{\mathbf{6}}$ in $17 \%$ and $4 \%$ yields respectively, giving a KIE of 4.3 by ${ }^{1} \mathrm{H}$ NMR spectroscopy.

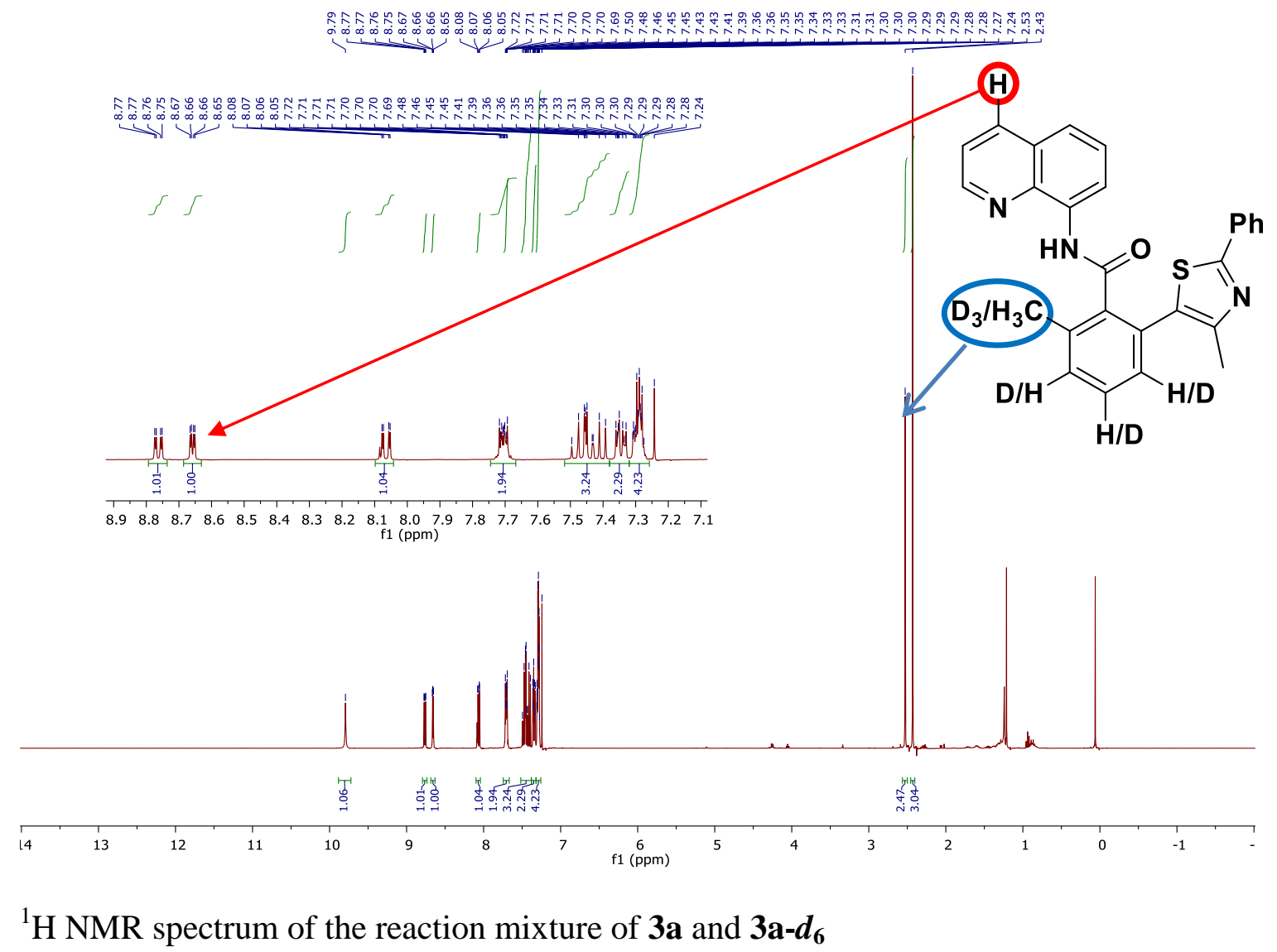

Procedure for the Isotopic Exchange Experiment. An oven-dried $50 \mathrm{~mL}$ Schlenk tube with a stirring bar was charged with carboxylic acid $2(0.600 \mathrm{mmol})$, sodium carbonate (63 mg, $0.60 \mathrm{mmol})$, and dry DMA $(1 \mathrm{~mL})$. The reaction vessel was placed in a preheated oil bath and stirred for $0.5 \mathrm{~h}$ at $130{ }^{\circ} \mathrm{C}$. Solvent was removed under reduced pressure until dry and the resulting carboxylate salt was used without further purification. 
$\mathbf{1 a}-\boldsymbol{d}_{7}(53.8 \mathrm{mg}, 0.200 \mathrm{mmol}), \mathrm{Ni}(\mathrm{OAc})_{2} \bullet 4 \mathrm{H}_{2} \mathrm{O}(9.9 \mathrm{mg}, 0.040 \mathrm{mmol}), \mathrm{Ag}_{2} \mathrm{CO}_{3}(110 \mathrm{mg}$, $0.400 \mathrm{mmol}), \mathrm{Na}_{2} \mathrm{CO}_{3}(83 \mathrm{mg}, 0.80 \mathrm{mmol}), \mathrm{PivOH}(30.6 \mathrm{mg}, 0.300 \mathrm{mmol})$ were added, the tube was evacuated and backfilled with nitrogen three times after which DMA $(2 \mathrm{~mL})$ was added via syringe. The reaction mixture was stirred at $110{ }^{\circ} \mathrm{C}$ for $2 \mathrm{~h}$. Upon completion, the reaction tube was cooled to room temperature. The solution was diluted with ethyl acetate $(25 \mathrm{~mL})$ and poured into a $250 \mathrm{~mL}$ separatory funnel. To the solution, water (25 mL), $\mathrm{Na}_{2}$ EDTA (500 mg), aqueous $\mathrm{HCl}(1 \mathrm{~N}, 10 \mathrm{~mL})$ were added and extracted with ethyl acetate $(2 \times 30 \mathrm{~mL})$. The combined organic layers were washed with water and brine, dried $\mathrm{Na}_{2} \mathrm{SO}_{4}$, filtered and concentrated in vacuo. The residue was purified by silica gel column chromatography (gradient elution, hexanes:ethyl acetate $(19: 1, \mathrm{v} / \mathrm{v})$ to $(4: 1, \mathrm{v} / \mathrm{v}))$, yielding the corresponding product. The extent of proton incorporation was determined by ${ }^{1} \mathrm{H}$ NMR spectroscopy.

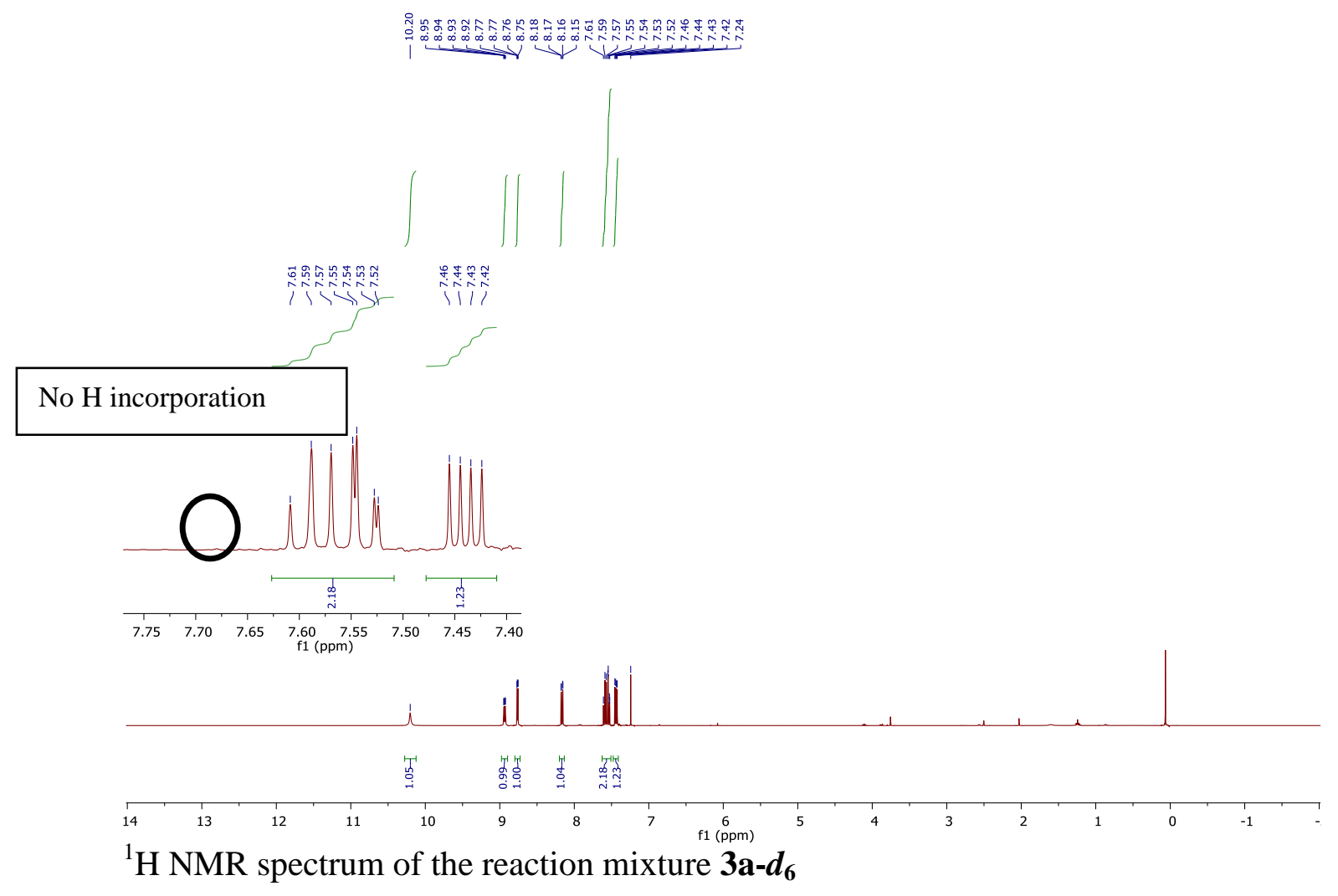




\section{General Procedure for the Synthesis of (Hetero)Aryl Benzamides (1)}

Procedure A: Synthesis from the benzoyl chloride. A $100 \mathrm{~mL}$ two-neck round bottom flask was charged with 8-aminoquinoline (1.44 g, $10.0 \mathrm{mmol}^{2}, \mathrm{Et}_{3} \mathrm{~N}$ (1.56 mL, 12.0 mmol), and methylene chloride $(20 \mathrm{~mL})$ under a $\mathrm{N}_{2}$ atmosphere. The corresponding acid chloride was added dropwise at room temperature over 10 minutes. The mixture was stirred for $6 \mathrm{~h}$ at room temperature. The reaction was quenched with $10 \mathrm{~mL}$ of saturated $\mathrm{NaHCO}_{3}$ and extracted with methylene chloride $(3 \times 25 \mathrm{~mL})$. The combined organic layer was washed with brine $(15 \mathrm{~mL})$ and dried over anhydrous $\mathrm{Na}_{2} \mathrm{SO}_{4}$. The solvent was removed under reduced pressure and the residue was purified via silica gel column chromatography (gradient elution, hexanes : ethyl acetate $(19: 1, \mathrm{v} / \mathrm{v})$ to $(4: 1, \mathrm{v} / \mathrm{v}))$.

Procedure B: Synthesis from the carboxylic acid. A $50 \mathrm{~mL}$ two-neck round bottom flask was charged with the corresponding carboxylic acid $(1.70 \mathrm{mmol}), \mathrm{N}, N$ dimethylformamide ( 2 drops), and methylene chloride $\left(2 \mathrm{~mL}\right.$ ) under a $\mathrm{N}_{2}$ atmosphere at 0 ${ }^{\circ} \mathrm{C}$. Oxalyl chloride $(0.17 \mathrm{~mL}, 2.0 \mathrm{mmol})$ was then added dropwise and the reaction mixture was allowed to stir for $3 \mathrm{~h}$ at room temperature. The solvent was removed under reduced pressure and the acid chloride was used without further purification. A $100 \mathrm{~mL}$ two-neck round bottom flask was charged with 8-aminoquinoline $(255 \mathrm{mg}, 1.70 \mathrm{mmol})$, $\mathrm{Et}_{3} \mathrm{~N}(0.278 \mathrm{~mL}, 2.00 \mathrm{mmol})$, and methylene chloride $(10 \mathrm{~mL})$ under $\mathrm{q}_{2}$ atmosphere at $0{ }^{\circ} \mathrm{C}$. The corresponding acid chloride was added dropwise to the reaction and stirred for $3 \mathrm{~h}$. The reaction was quenched with $5 \mathrm{~mL}$ of saturated $\mathrm{NaHCO}_{3}$ and extracted with methylene chloride three times $(3 \times 25 \mathrm{~mL})$. The combined organic layer was washed with brine $(15 \mathrm{~mL})$ and dried over anhydrous $\mathrm{Na}_{2} \mathrm{SO}_{4}$. The solvent was removed under reduced pressure and the residue was purified via silica gel column chromatography (gradient elution, hexanes : ethyl acetate $(19: 1, \mathrm{v} / \mathrm{v})$ to $(9: 1, \mathrm{v} / \mathrm{v}))$.

\section{General Procedure for the Synthesis of Thiazole Carboxylic Acids (2)}

A mixture of thiobenzamide $(3.77 \mathrm{~g}, 27.5 \mathrm{mmol})$ and ethyl 2-chloroacetoacetate $(3.45$ $\mathrm{mL}, 25.0 \mathrm{mmol}$ ) in methanol was refluxed for 5-6 h in a $100 \mathrm{~mL}$ round bottom flask. The 
solvent was removed under reduced pressure to yield the corresponding crude ethyl carboxylate. A $250 \mathrm{~mL}$ round bottom flask was charged with the crude ethyl carboxylate, $\mathrm{THF} / \mathrm{EtOH} / \mathrm{H}_{2} \mathrm{O}(1: 1: 1,165 \mathrm{~mL}, \mathrm{v} / \mathrm{v})$, and $\mathrm{KOH}$ (4 equiv) and the mixture was allowed to stir at room temperature overnight. The reaction was quenched with $\mathrm{HCl}(2 \mathrm{~N})$ until a $\mathrm{pH}$ of 2 was reached. The solid was filtered and dried under reduced pressure to yield the corresponding carboxylic acid.

\section{Characterization of (Hetero)Aryl Benzamides (1)}<smiles>Cc1ccccc1C(=O)Nc1cccc2cccnc12</smiles>

2-methyl- $N$-(quinolin-8-yl) benzamide (1a): The following compound was synthesized following General Procedure A. ${ }^{1} \mathrm{H}$ NMR $\left(400 \mathrm{MHz}, \mathrm{CDCl}_{3}\right) \delta 10.21$ (s, 1H), 8.95 (dd, $J$ $=7.5,1.5 \mathrm{~Hz}, 1 \mathrm{H}), 8.78(\mathrm{dd}, J=4.2,1.7 \mathrm{~Hz}, 1 \mathrm{H}), 8.19(\mathrm{dd}, J=8.3,1.7 \mathrm{~Hz}, 1 \mathrm{H}), 7.69(\mathrm{dd}$, $J=7.9,1.4 \mathrm{~Hz}, 1 \mathrm{H}), 7.64-7.53(\mathrm{~m}, 2 \mathrm{H}), 7.50-7.37$ (m, 3H), 7.33 (tt, $J=7.7,0.8 \mathrm{~Hz}$, $2 \mathrm{H}), 2.61(\mathrm{~s}, 3 \mathrm{H}) .{ }^{13} \mathrm{C}\left\{{ }^{1} \mathrm{H}\right\}$ NMR $\left(100 \mathrm{MHz}, \mathrm{CDCl}_{3}\right) \delta 168.17,148.28,138.60,136.70$, 136.64, 136.35, 134.76, 131.41, 130.36, 128.00, 127.40, 127.27, 126.05, 121.81, 121.69, $116.49,20.27$. The spectral data are consistent with those reported in the literature. ${ }^{46}$<smiles>O=C(Nc1cccc2cccnc12)c1ccccc1</smiles>

$N$-(quinolin-8-yl) benzamide (1z): The following compound was synthesized following General Procedure A. ${ }^{1} \mathrm{H}$ NMR (400 MHz, $\left.\mathrm{CDCl}_{3}\right) \delta 10.73$ (s, $1 \mathrm{H}$ ), 8.95 (dd, $J=7.5,1.4$ $\mathrm{Hz}, 1 \mathrm{H}), 8.82(\mathrm{dd}, J=4.2,1.7 \mathrm{~Hz}, 1 \mathrm{H}), 8.14(\mathrm{dd}, J=8.3,1.7 \mathrm{~Hz}, 1 \mathrm{H}), 8.12-8.06(\mathrm{~m}$, $2 \mathrm{H}), 7.62-7.48(\mathrm{~m}, 5 \mathrm{H}), 7.44(\mathrm{dd}, J=8.3,4.2 \mathrm{~Hz}, 1 \mathrm{H}) .{ }^{13} \mathrm{C}\left\{{ }^{1} \mathrm{H}\right\} \mathrm{NMR}(100 \mathrm{MHz}$, $\left.\mathrm{CDCl}_{3}\right) \delta 165.47,148.33,138.81,136.43,135.21,134.64,131.90,128.86,128.04$, 
$127.49,127.35,121.76,121.74,116.59$. The spectral data are consistent with those reported in the literature. ${ }^{46}$<smiles>Cc1cccc(C(=O)Nc2cccc3cccnc23)c1</smiles>

3-methyl- $N$-(quinolin-8-yl) benzamide (1aa): The following compound was synthesized following General Procedure A. ${ }^{1} \mathrm{H}$ NMR (400 MHz, $\left.\mathrm{CDCl}_{3}\right) \delta 10.67(\mathrm{~s}, 1 \mathrm{H})$, $8.94(\mathrm{dd}, J=7.6,1.2 \mathrm{~Hz}, 1 \mathrm{H}), 8.79(\mathrm{dd}, J=4.2,3.7,1.7 \mathrm{~Hz}, 1 \mathrm{H}), 8.08(\mathrm{dd}, J=8.2,4.5$, $1.7 \mathrm{~Hz}, 1 \mathrm{H}), 7.90-7.82(\mathrm{~m}, 2 \mathrm{H}), 7.54(\mathrm{td}, J=7.9,2.8 \mathrm{~Hz}, 1 \mathrm{H}), 7.46(\mathrm{ddd}, J=8.3,3.5$, $1.4 \mathrm{~Hz}, 1 \mathrm{H}), 7.44-7.32(\mathrm{~m}, 3 \mathrm{H}), 2.45(\mathrm{~d}, J=2.3 \mathrm{~Hz}, 3 \mathrm{H}) .{ }^{13} \mathrm{C}\left\{{ }^{1} \mathrm{H}\right\} \mathrm{NMR}(100 \mathrm{MHz}$, $\left.\mathrm{CDCl}_{3}\right) \delta 165.50,148.19,138.66,138.58,136.26,135.07,134.57,132.55,128.59$, $127.99,127.89,127.33,124.16,121.59,116.43,21.45$. The spectral data are consistent with those reported in the literature. ${ }^{47}$<smiles>COc1cccc(C(=O)Nc2cccc3cccnc23)c1</smiles>

3-methoxy- $N$-(quinolin-8-yl) benzamide (1ab): The following compound was synthesized following General Procedure A. ${ }^{1} \mathrm{H}$ NMR $\left(400 \mathrm{MHz}, \mathrm{CDCl}_{3}\right) \delta 10.73(\mathrm{~s}, 1 \mathrm{H})$, $8.93(\mathrm{dd}, J=7.5,1.1 \mathrm{~Hz}, 1 \mathrm{H}), 8.83(\mathrm{dd}, J=4.2,2.1 \mathrm{~Hz}, 1 \mathrm{H}), 8.16$ (dd, $1 \mathrm{H}), 7.64$ (tdd, $J=$ 3.3, 1.7, 1.0 Hz, 2H), 7.62 - $7.56(\mathrm{~m}, 1 \mathrm{H}), 7.56-7.51(\mathrm{~m}, 1 \mathrm{H}), 7.50-7.41(\mathrm{~m}, 2 \mathrm{H}), 7.12$ $(\mathrm{dd}, J=8.2,2.5,1.0 \mathrm{~Hz}, 1 \mathrm{H}), 3.91(\mathrm{~s}, 3 \mathrm{H}) .{ }^{13} \mathrm{C}\left\{{ }^{1} \mathrm{H}\right\} \mathrm{NMR}\left(100 \mathrm{MHz}, \mathrm{CDCl}_{3}\right) \delta$ 165.08, 159.96, 148.26, 148.24, 138.63, 136.54, 136.29, 136.26, 134.48, 129.74, 127.89, 127.35, 127.31, 121.69, 121.64, 119.01, 118.99, 117.90, 117.84, 116.45, 116.41, 112.72, 112.71, 55.43. The spectral data are consistent with those reported in the literature. ${ }^{48}$ 
<smiles>O=C(Nc1cccc2cccnc12)c1cccc(Cl)c1</smiles>

3-chloro- $N$-(quinolin-8-yl) benzamide (1ac): The following compound was synthesized following General Procedure A. ${ }^{1} \mathrm{H}$ NMR (400 MHz, $\left.\mathrm{CDCl}_{3}\right) \delta 10.67$ (s, 1H), 8.89 (dd, $J$ $=7.3,1.8 \mathrm{~Hz}, 1 \mathrm{H}), 8.83(\mathrm{dd}, J=4.1,2.0 \mathrm{~Hz}, 1 \mathrm{H}), 8.16(\mathrm{dd}, J=8.3,3.5,1.6 \mathrm{~Hz}, 1 \mathrm{H}), 8.05$ $(\mathrm{d}, J=2.0 \mathrm{~Hz}, 1 \mathrm{H}), 7.93(\mathrm{dd}, J=7.7,1.6 \mathrm{~Hz}, 1 \mathrm{H}), 7.61-7.50(\mathrm{~m}, 3 \mathrm{H}), 7.46(\mathrm{td}, J=7.4$, $2.8 \mathrm{~Hz}, 2 \mathrm{H}) .{ }^{13} \mathrm{C}\left\{{ }^{1} \mathrm{H}\right\} \mathrm{NMR}\left(100 \mathrm{MHz}, \mathrm{CDCl}_{3}\right) \delta 163.08,147.93,138.12,136.33,135.88$, 134.59 , 133.75, 131.40, 129.63, 127.46, 127.34, 126.85, 124.71, 121.63, 121.35, 116.17 . The spectral data are consistent with those reported in the literature. ${ }^{47}$<smiles>O=C(Nc1cccc2cccnc12)c1cccc(Br)c1</smiles>

3-bromo- $N$-(quinolin-8-yl) benzamide (1ad): The following compound was synthesized following General Procedure A. ${ }^{1} \mathrm{H}$ NMR $\left(400 \mathrm{MHz}, \mathrm{CDCl}_{3}\right) \delta 10.60(\mathrm{~s}, 1 \mathrm{H})$, $8.84(\mathrm{dd}, J=7.4,1.6 \mathrm{~Hz}, 1 \mathrm{H}), 8.78(\mathrm{dd}, J=4.2,1.7 \mathrm{~Hz}, 1 \mathrm{H}), 8.16(\mathrm{t}, J=1.8 \mathrm{~Hz}, 1 \mathrm{H})$, $8.09(\mathrm{dd}, J=8.3,1.7 \mathrm{~Hz}, 1 \mathrm{H}), 7.92(\mathrm{ddd}, J=7.8,1.7,1.0 \mathrm{~Hz}, 1 \mathrm{H}), 7.63(\mathrm{ddd}, J=8.0,2.0$, $1.0 \mathrm{~Hz}, 1 \mathrm{H}), 7.56-7.43(\mathrm{~m}, 2 \mathrm{H}), 7.40(\mathrm{dd}, J=8.3,4.2 \mathrm{~Hz}, 1 \mathrm{H}), 7.34(\mathrm{t}, J=7.9 \mathrm{~Hz}, 1 \mathrm{H})$. ${ }^{13} \mathrm{C}\left\{{ }^{1} \mathrm{H}\right\}$ NMR $\left(100 \mathrm{MHz}, \mathrm{CDCl}_{3}\right) \delta 163.72,148.38,138.65,137.06,136.38,134.77$, $134.21,130.64,130.29,127.94,127.36,125.65,123.07,122.03,121.77,116.67$. The spectral data are consistent with those reported in the literature. ${ }^{47}$ 
<smiles>O=C(Nc1cccc2cccnc12)c1cccc2ccccc12</smiles>

$N$-(quinolin-8-yl)naphthalene-1-carboxamide (1ae): The following compound was synthesized following General Procedure A. ${ }^{1} \mathrm{H}$ NMR $\left(400 \mathrm{MHz}, \mathrm{CDCl}_{3}\right) \delta 10.45(\mathrm{~s}, 1 \mathrm{H})$, 9.09 (dd, $J=7.7,1.3 \mathrm{~Hz}, 1 \mathrm{H}), 8.74(\mathrm{dd}, J=4.2,1.7 \mathrm{~Hz}, 1 \mathrm{H}), 8.61-8.55(\mathrm{~m}, 1 \mathrm{H}), 8.16$ $(\mathrm{dd}, J=8.3,1.7 \mathrm{~Hz}, 1 \mathrm{H}), 8.00(\mathrm{dt}, J=8.2,1.1 \mathrm{~Hz}, 1 \mathrm{H}), 7.93(\mathrm{ddd}, J=7.3,5.5,1.6 \mathrm{~Hz}$, $2 \mathrm{H}), 7.65(\mathrm{t}, J=7.9 \mathrm{~Hz}, 1 \mathrm{H}), 7.63-7.53(\mathrm{~m}, 4 \mathrm{H}), 7.42(\mathrm{dd}, J=8.3,4.2 \mathrm{~Hz}, 1 \mathrm{H}) .{ }^{13} \mathrm{C}\left\{{ }^{1} \mathrm{H}\right\}$ NMR $\left(100 \mathrm{MHz}, \mathrm{CDCl}_{3}\right) \delta 167.78,148.37,138.68,136.41,134.90,133.96,131.21$, $130.43,128.47,128.08,127.49,127.39$, 127.38, 126.59, 125.66, 125.59, 124.94, 122.03, $121.76,116.78$. The spectral data are consistent with those reported in the literature. ${ }^{47}$<smiles>Cn1cccc1C(=O)Nc1cccc2cccnc12</smiles>

1-methyl-N-(quinolin-8-yl)-1H-pyrrole-2-carboxamide (1af): The following compound was synthesized following General Procedure B. ${ }^{1} \mathrm{H}$ NMR (400 $\mathrm{MHz}, \mathrm{CDCl}_{3}$ ) $\delta 10.43(\mathrm{~s}, 1 \mathrm{H}), 8.86-8.74(\mathrm{~m}, 2 \mathrm{H}), 8.13(\mathrm{dd}, J=8.3,1.7 \mathrm{~Hz}, 1 \mathrm{H}), 7.55(\mathrm{t}, J=7.9 \mathrm{~Hz}$, $1 \mathrm{H}), 7.47(\mathrm{dd}, J=8.3,1.4 \mathrm{~Hz}, 1 \mathrm{H}), 7.43(\mathrm{dd}, J=8.2,4.2 \mathrm{~Hz}, 1 \mathrm{H}), 7.02(\mathrm{dd}, J=4.0,1.7$ $\mathrm{Hz}, 1 \mathrm{H}), 6.81(\mathrm{t}, J=2.1 \mathrm{~Hz}, 1 \mathrm{H}), 6.21(\mathrm{dd}, J=4.0,2.5 \mathrm{~Hz}, 1 \mathrm{H}), 4.06(\mathrm{~s}, 3 \mathrm{H}) .{ }^{13} \mathrm{C}\left\{{ }^{1} \mathrm{H}\right\}$ NMR $\left(100 \mathrm{MHz}, \mathrm{CDCl}_{3}\right) \delta 160.11,148.21,138.65,136.34,135.13,128.90,127.47$, $126.34,121.65,121.00,115.86,115.85,113.09,107.67,37.03$. The spectral data are consistent with those reported in the literature. ${ }^{47}$

\section{Characterization of Thizaole Carboxylic Acids (2).}




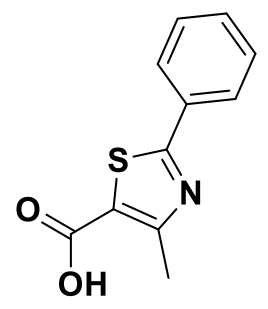

4-methyl-2-phenyl-1,3-thiazole-5-carboxylic acid (2a). ${ }^{1} \mathrm{H}$ NMR (400 MHz, DMSO- $d_{6}$ ) $\delta 13.30(\mathrm{~s}, 1 \mathrm{H}), 7.93(\mathrm{~d}, J=8.0 \mathrm{~Hz}, 2 \mathrm{H}), 7.63-7.39$ (m, 3H), 2.67 (s, 3H). ${ }^{13} \mathrm{C}\left\{{ }^{1} \mathrm{H}\right\}$ NMR (100 MHz, DMSO- $\left.d_{6}\right) \delta 168.44,162.92,159.61,132.36,131.18,129.29,126.47$, 122.91, 17.14. The spectral data are consistent with those reported in the literature. ${ }^{49}$<smiles>Cc1nc(-c2ccc(Cl)cc2)sc1C(=O)O</smiles>

2-(4-chlorophenyl)-4-methyl-1,3-thiazole-5-carboxylic acid (2b). ${ }^{1} \mathrm{H}$ NMR $(400 \mathrm{MHz}$, DMSO- $\left.d_{6}\right) \delta 13.42(\mathrm{~s}, 1 \mathrm{H}), 7.94(\mathrm{~d}, J=8.6 \mathrm{~Hz}, 2 \mathrm{H}), 7.53$ (d, $\left.J=8.5 \mathrm{~Hz}, 2 \mathrm{H}\right), 2.66$ (s, $3 \mathrm{H}) .{ }^{13} \mathrm{C}\left\{{ }^{1} \mathrm{H}\right\}$ NMR (100 MHz, DMSO- $\left.d_{6}\right) \delta 166.86,162.70,159.51,135.73,131.04$, $129.15,127.94,123.23,16.96$. The spectral data are consistent with those reported in the literature. $^{49}$

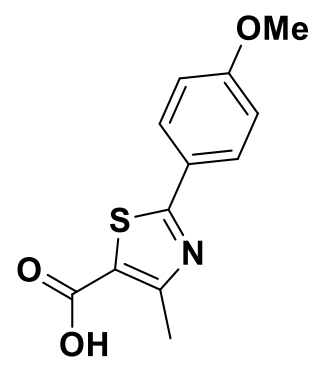

2-(4-methoxyphenyl)-4-methyl-1,3-thiazole-5-carboxylic acid (2c). ${ }^{1} \mathrm{H}$ NMR (400 MHz, DMSO- $\left.d_{6}\right) \delta 13.26(\mathrm{~s}, 1 \mathrm{H}), 7.87(\mathrm{~d}, J=8.8 \mathrm{~Hz}, 2 \mathrm{H}), 7.05(\mathrm{~d}, J=8.6 \mathrm{~Hz}, 2 \mathrm{H}), 3.83$ (s, 3H), $2.64(\mathrm{~s}, 3 \mathrm{H}) .{ }^{13} \mathrm{C}\left\{{ }^{1} \mathrm{H}\right\}$ NMR (100 MHz, DMSO- $\left.d_{6}\right) \delta 168.44,163.07,161.63$, $159.61,128.14,125.16,121.69,114.56,55.40,17.13$. The spectral data are consistent with those reported in the literature. ${ }^{50}$ 


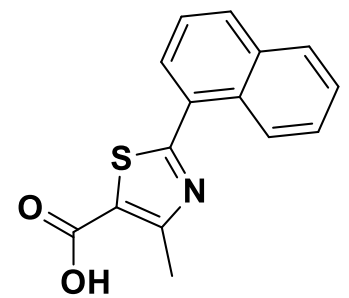

4-methyl-2-(naphthalene-1-yl)-1,3-thiazole-5-carboxylic acid (2d): White solid, $\mathrm{mp}=$ $207-209{ }^{\circ} \mathrm{C} .{ }^{1} \mathrm{H}$ NMR (400 MHz, DMSO-d 6 ) $\delta 13.44$ (s, 1H), 8.81 (d, 1H), 8.08 (dd, $J=$ 8.4, 2.1, $1.0 \mathrm{~Hz}, 1 \mathrm{H}), 8.01(\mathrm{~d}, 1 \mathrm{H}), 7.90$ (dt, $J=7.2,1.7 \mathrm{~Hz}, 1 \mathrm{H}), 7.66-7.55(\mathrm{~m}, 3 \mathrm{H})$, $2.76(\mathrm{~s}, 3 \mathrm{H}) .{ }^{13} \mathrm{C}\left\{{ }^{1} \mathrm{H}\right\} \mathrm{NMR}\left(100 \mathrm{MHz}, \mathrm{DMSO}-d_{6}\right) \delta 168.48,163.05,159.35,133.67$, 131.22 , 129.62, 129.36, 128.92, 128.53, 127.62, 126.56, 125.49, 125.34, 123.60, 17.32. FTIR (ATR, $\mathrm{cm}^{-1}$ ): 3330, 3156, 1690, 1614, 1421, 1258, 911. HRMS (ESI-MS) m/z calcd for $\mathrm{C}_{15} \mathrm{H}_{12} \mathrm{NO}_{2} \mathrm{~S}[\mathrm{M}+\mathrm{H}]^{+} 270.0589$ found 270.0595 .

Characterization of Ni-Catalyzed Oxidative Decarboxylative (Hetero) Arylation Products.<smiles>Cc1cccc(-c2sc(-c3ccccc3)nc2C)c1C(=O)Nc1cccc2cccnc12</smiles>

2-methyl-6-(4-methyl-2-phenyl-1,3-thiazol-5-yl)- $N$-(quinolin-8-yl)benzamide

(3a):

The title compound was synthesized following General Procedure A. Light yellow solid in $93 \%(81.0 \mathrm{mg}, 0.18 \mathrm{mmol}), \mathrm{mp}=146-148{ }^{\circ} \mathrm{C} .{ }^{1} \mathrm{H} \mathrm{NMR}\left(400 \mathrm{MHz}, \mathrm{CDCl}_{3}\right): \delta 9.83$ (s, 1H), $8.80(\mathrm{dd}, J=7.3,1.7 \mathrm{~Hz}, 1 \mathrm{H}), 8.67$ (dd, $J=4.2,1.7 \mathrm{~Hz}, 1 \mathrm{H}), 8.05$ (dd, $J=8.3$, $1.7 \mathrm{~Hz}, 1 \mathrm{H}), 7.77-7.69(\mathrm{~m}, 2 \mathrm{H}), 7.52-7.39$ (m, 3H), $7.39-7.27$ (m, 6H), 2.55 (s, 3H), $2.46(\mathrm{~s}, 3 \mathrm{H}) .{ }^{13} \mathrm{C}\left\{{ }^{1} \mathrm{H}\right\} \mathrm{NMR}\left(100 \mathrm{MHz}, \mathrm{CDCl}_{3}\right): \delta 167.11,166.18,150.90,148.02$, $138.68,138.30,136.18,134.14,133.45,130.89,129.63,129.20,129.18,129.17,129.02$, 128.65, 127.84, 127.23, 126.23, 121.91, 121.52, 116.59, 19.82, 15.97. FTIR (ATR, $\mathrm{cm}^{-1}$ ): 
3339, 3061, 2921, 1670, 1580, 1520, 1483, 1384, 1325, 905, 726. HRMS (ESI-MS) m/z calcd for $\mathrm{C}_{27} \mathrm{H}_{22} \mathrm{~N}_{3} \mathrm{OS}[\mathrm{M}+\mathrm{H}]^{+} 436.1484$ found 436.1497 .

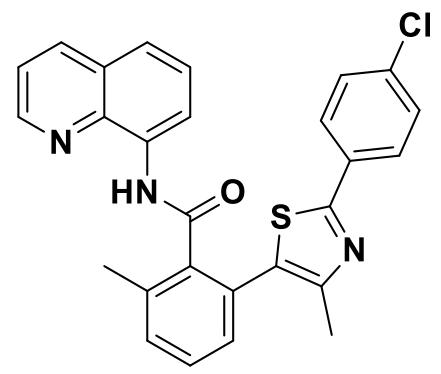

\section{2-[2-(4-chlorophenyl)-4-methyl-1,3-thiazol-5-yl]-6-methyl- $N$-(quinolin-8-}

yl)benzamide (3b): The title compound was synthesized following General Procedure A. Light yellow solid in $89 \%$ (83.7 mg, $0.17 \mathrm{mmol}), \mathrm{mp}=80-82{ }^{\circ} \mathrm{C} .{ }^{1} \mathrm{H} \mathrm{NMR}(400 \mathrm{MHz}$, $\left.\mathrm{CDCl}_{3}\right): \delta 9.82(\mathrm{~s}, 1 \mathrm{H}), 8.77(\mathrm{dd}, J=6.9,2.1 \mathrm{~Hz}, 1 \mathrm{H}), 8.68(\mathrm{dd}, J=4.2,1.7 \mathrm{~Hz}, 1 \mathrm{H}), 8.10$ (dd, $J=8.3,1.7 \mathrm{~Hz}, 1 \mathrm{H}), 7.69-7.63(\mathrm{~m}, 2 \mathrm{H}), 7.53-7.45(\mathrm{~m}, 2 \mathrm{H}), 7.42(\mathrm{~d}, J=7.6 \mathrm{~Hz}$, 1H), $7.41-7.35(\mathrm{~m}, 2 \mathrm{H}), 7.33-7.24(\mathrm{~m}, 3 \mathrm{H}), 2.54(\mathrm{~s}, 3 \mathrm{H}), 2.44(\mathrm{~s}, 3 \mathrm{H}) .{ }^{13} \mathrm{C}\left\{{ }^{1} \mathrm{H}\right\} \mathrm{NMR}$ $\left(100 \mathrm{MHz}, \mathrm{CDCl}_{3}\right): \delta 167.22,165.04,151.11,148.13,138.82,138.35,136.54,136.39$, 135.81, 134.18, 131.91, 131.21, 129.59, 129.39, 129.29, 129.07, 129.00, 128.04, 127.63, 127.47, 122.16, 121.71, 116.94, 19.95, 15.99. FTIR (ATR, $\left.\mathrm{cm}^{-1}\right): 3343,3188,2943$, $1674,1523,1483,1424,1326,826,789$. HRMS (ESI-MS) m/z calcd for $\mathrm{C}_{27} \mathrm{H}_{21} \mathrm{ClN}_{3} \mathrm{OS}$ $[\mathrm{M}+\mathrm{H}]^{+} 470.1094$ found 470.0925 .<smiles>COc1ccc(-c2nc(C)c(-c3cccc(C)c3C(=O)Nc3cccc4cccnc34)s2)cc1</smiles>

\section{2-[2-(4-methoxyphenyl)-4-methyl-1,3-thiazol-5-yl]-6-methyl- $N$-(quinolin-8-}

yl)benzamide (3c): The title compound was synthesized following General Procedure A. Light yellow solid in $72 \%(67.0 \mathrm{mg}, 0.14 \mathrm{mmol}), \mathrm{mp}=85-87^{\circ} \mathrm{C} .{ }^{1} \mathrm{H}$ NMR $(400 \mathrm{MHz}$, 
$\left.\mathrm{CDCl}_{3}\right): \delta 9.82(\mathrm{~s}, 1 \mathrm{H}), 8.79(\mathrm{dt}, J=7.4,1.3 \mathrm{~Hz}, 1 \mathrm{H}), 8.66(\mathrm{ddd}, J=4.1,1.7,0.7 \mathrm{~Hz}, 1 \mathrm{H})$, $8.09-7.99(\mathrm{~m}, 1 \mathrm{H}), 7.71-7.61(\mathrm{~m}, 2 \mathrm{H}), 7.53-7.38(\mathrm{~m}, 3 \mathrm{H}), 7.38-7.28(\mathrm{~m}, 3 \mathrm{H}), 6.86$ - $6.75(\mathrm{~m}, 2 \mathrm{H}), 3.77$ (s, 3H), 2.54 (s, 3H), 2.44 (s, 3H). ${ }^{13} \mathrm{C}\left\{{ }^{1} \mathrm{H}\right\}$ NMR $(100 \mathrm{MHz}$, $\left.\mathrm{CDCl}_{3}\right): \delta 167.28,166.22,160.93,150.64,148.12,138.78,138.41,136.27,136.25$, $134.28,130.88,129.44,129.35,129.25,128.09,127.94,127.79,127.33,126.54,121.98$, 121.61, 116.68, 114.10, 55.38, 19.92, 16.06. FTIR (ATR, $\mathrm{cm}^{-1}$ ): 3333, 3062, 2926, 1671, 1518, 1482, 1460, 1423, 1325, 1252, 1171, 905, 726. HRMS (ESI-MS) m/z calcd for $\mathrm{C}_{28} \mathrm{H}_{24} \mathrm{~N}_{3} \mathrm{O}_{2} \mathrm{~S}[\mathrm{M}+\mathrm{H}]^{+} 466.1589$ found 466.1578 .

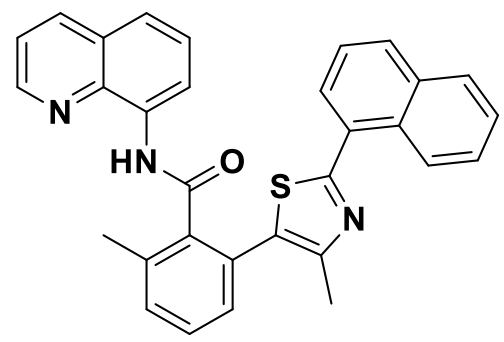

\section{2-methyl-6-[4-methyl-2-(naphthalene-1-yl)-1,3-thiazol-5-yl]- $N$-(quinolin-8-}

yl)benzamide (3d): The title compound was synthesized following General Procedure A. Light yellow solid in $93 \%$ (90.3 mg, $0.18 \mathrm{mmol}), \mathrm{mp}=208-210{ }^{\circ} \mathrm{C}$. ${ }^{1} \mathrm{H}$ NMR (400 $\left.\mathrm{MHz}, \mathrm{CDCl}_{3}\right): \delta 9.87(\mathrm{~s}, 1 \mathrm{H}), 8.87(\mathrm{dd}, J=7.5,1.5 \mathrm{~Hz}, 1 \mathrm{H}), 8.68(\mathrm{dd}, J=4.2,1.7 \mathrm{~Hz}$, $1 \mathrm{H}), 8.07(\mathrm{ddd}, J=8.3,6.8,1.3 \mathrm{~Hz}, 2 \mathrm{H}), 7.79(\mathrm{ddt}, J=9.2,8.1,1.0 \mathrm{~Hz}, 2 \mathrm{H}), 7.57-7.30$ (m, 9H), 7.21 (ddd, $J=8.4,6.8,1.4 \mathrm{~Hz}, 1 \mathrm{H}), 2.60$ (s, 3H), 2.59 (s, 3H). ${ }^{13} \mathrm{C}\left\{{ }^{1} \mathrm{H}\right\} \mathrm{NMR}$ $\left(100 \mathrm{MHz}, \mathrm{CDCl}_{3}\right): \delta 167.27,165.41,150.73,148.17,138.70,138.34,136.69,136.32$, 134.34, 133.80, 131.09, 130.79, 130.65, 130.19, 130.05, 129.43, 129.30, 129.15, 128.18, $128.15,127.96,127.44,127.03$, 126.12, 125.51, 124.89, 122.01, 121.72, 116.59, 20.03, 16.19. FTIR (ATR, $\mathrm{cm}^{-1}$ ): 3339, 3057, 2919, 1672, 1519, 1482, 1423, 1325, 1263, 906, 729. HRMS (ESI-MS) m/z calcd for $\mathrm{C}_{31} \mathrm{H}_{24} \mathrm{~N}_{3} \mathrm{OS}[\mathrm{M}+\mathrm{H}]^{+} 486.1640$ found 486.1650 . 


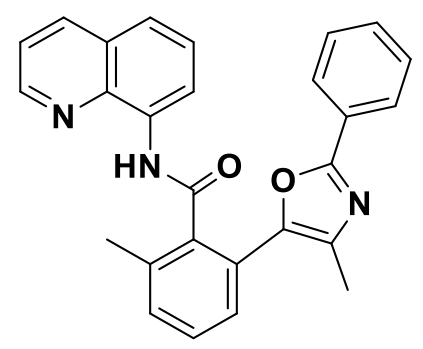

2-methyl-6-(4-methyl-2-phenyl-1,3-oxazol-5-yl)- $N$-(quinolin-8-yl)benzamide (3e): The title compound was synthesized following General Procedure A. White solid in $74 \%$ (62.0 mg, $0.14 \mathrm{mmol}), \mathrm{mp}=148-150{ }^{\circ} \mathrm{C} .{ }^{1} \mathrm{H} \mathrm{NMR}\left(400 \mathrm{MHz}, \mathrm{CDCl}_{3}\right): \delta 9.92(\mathrm{~s}, 1 \mathrm{H})$, 8.87 (dd, $J=7.2,1.8 \mathrm{~Hz}, 1 \mathrm{H}), 8.62$ (dd, $J=4.2,1.7 \mathrm{~Hz}, 1 \mathrm{H}), 8.08$ (dd, $J=8.3,1.7 \mathrm{~Hz}$, 1H), $7.65-7.58(\mathrm{~m}, 2 \mathrm{H}), 7.52-7.39(\mathrm{~m}, 4 \mathrm{H}), 7.38-7.30(\mathrm{~m}, 2 \mathrm{H}), 7.21-7.15(\mathrm{~m}, 1 \mathrm{H})$, $7.05-6.98(\mathrm{~m}, 2 \mathrm{H}), 2.56(\mathrm{~s}, 3 \mathrm{H}), 2.39(\mathrm{~s}, 3 \mathrm{H}) .{ }^{13} \mathrm{C}\left\{{ }^{1} \mathrm{H}\right\}$ NMR $\left(100 \mathrm{MHz}, \mathrm{CDCl}_{3}\right): \delta$ $167.98,160.09$, 148.33, 144.30, 138.54, 136.77, 136.33, 136.23, 135.24, 134.47, 130.84, $129.90,129.43,128.33,128.01,127.39,126.99,126.30,126.28,126.12$, 122.01, 121.71, 117.06, 19.86, 13.10. FTIR (ATR, $\mathrm{cm}^{-1}$ ): 3346, 3079, 2924, 1677, 1519, 1482, 1424, 1325, 1264, 1028, 898, 711. HRMS (ESI-MS) m/z calcd for $\mathrm{C}_{27} \mathrm{H}_{22} \mathrm{~N}_{3} \mathrm{O}_{2}[\mathrm{M}+\mathrm{H}]^{+}$ 420.1712 found 420.1728 .

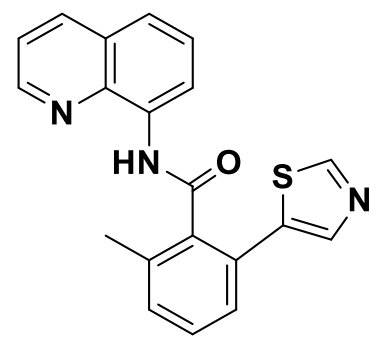

2-methyl- $N$-(quinolin-8-yl)-6-(1,3-thiazol-5-yl)benzamide (3f): The title compound was synthesized following General Procedure B. Bright yellow solid in $90 \%(62.1 \mathrm{mg}$, $0.18 \mathrm{mmol}), \mathrm{mp}=56-58{ }^{\circ} \mathrm{C} .{ }^{1} \mathrm{H} \mathrm{NMR}\left(400 \mathrm{MHz}, \mathrm{CDCl}_{3}\right): \delta 9.86(\mathrm{~s}, 1 \mathrm{H}), 8.86(\mathrm{dd}, J=$ 7.1, $1.9 \mathrm{~Hz}, 1 \mathrm{H}), 8.67$ (dd, $J=4.2,1.7 \mathrm{~Hz}, 1 \mathrm{H}), 8.62$ (d, $J=0.6 \mathrm{~Hz}, 1 \mathrm{H}), 8.13$ (dd, $J=$ 8.3, $1.7 \mathrm{~Hz}, 1 \mathrm{H}), 8.02(\mathrm{t}, J=0.5 \mathrm{~Hz}, 1 \mathrm{H}), 7.59-7.49(\mathrm{~m}, 2 \mathrm{H}), 7.44-7.37(\mathrm{~m}, 3 \mathrm{H}), 7.36$ - $7.31(\mathrm{~m}, 1 \mathrm{H}), 2.52(\mathrm{~s}, 3 \mathrm{H}) .{ }^{13} \mathrm{C}\left\{{ }^{1} \mathrm{H}\right\} \mathrm{NMR}\left(100 \mathrm{MHz}, \mathrm{CDCl}_{3}\right): \delta 167.64,153.37$, $148.35,141.91,138.50,137.32$, 136.46, 136.33, 136.17, 134.16, 130.90, 129.56, 128.40, 128.25, 128.05, 127.46, 122.34, 121.77, 117.13, 19.75. FTIR (ATR, $\left.\mathrm{cm}^{-1}\right): 3339,3003$, 
2916, 1672, 1519, 1483, 1424, 1386, 1325, 874, 789, 754. HRMS (ESI-MS) m/z calcd for $\mathrm{C}_{20} \mathrm{H}_{16} \mathrm{~N}_{3} \mathrm{OS}[\mathrm{M}+\mathrm{H}]^{+} 346.1014$ found 346.1028 .<smiles>Cc1cccc(-c2ccnn2C)c1C(=O)Nc1cccc2cccnc12</smiles>

2-methyl-6-(1-methyl-1H-pyrazol-5-yl)- $N$-(quinolin-8-yl)benzamide (3g): The title compound was synthesized following General Procedure B. Light yellow solid in $74 \%$ (50.6 mg, $0.14 \mathrm{mmol}), \mathrm{mp}=140-142{ }^{\circ} \mathrm{C} .{ }^{1} \mathrm{H}$ NMR $\left(400 \mathrm{MHz}, \mathrm{CDCl}_{3}\right): \delta 9.69(\mathrm{~s}, 1 \mathrm{H})$, $8.74(\mathrm{dd}, J=6.3,2.7 \mathrm{~Hz}, 1 \mathrm{H}), 8.69(\mathrm{dd}, J=4.2,1.7 \mathrm{~Hz}, 1 \mathrm{H}), 8.13(\mathrm{dd}, J=8.3,1.7 \mathrm{~Hz}$, 1H), $7.55-7.47(\mathrm{~m}, 2 \mathrm{H}), 7.47-7.36(\mathrm{~m}, 3 \mathrm{H}), 7.28-7.20(\mathrm{~m}, 2 \mathrm{H}), 6.33(\mathrm{~d}, J=1.9 \mathrm{~Hz}$, 1H), $3.84(\mathrm{~s}, 3 \mathrm{H}), 2.54(\mathrm{~s}, 3 \mathrm{H}) .{ }^{13} \mathrm{C}\left\{{ }^{1} \mathrm{H}\right\}$ NMR $\left(100 \mathrm{MHz}, \mathrm{CDCl}_{3}\right): \delta$ 167.13, 148.15, $140.83,138.45,138.33,136.42$, 136.21, 134.10, 131.26, 129.22, 128.44, 127.99, 127.97, 127.37, 122.07, 121.72, 116.66, 107.38, 37.24, 19.77. FTIR (ATR, $\mathrm{cm}^{-1}$ ): 3326, 3067, 2922, 1675, 1523, 1483, 1424, 1326, 826, 753. HRMS (ESI-MS) $\mathrm{m} / \mathrm{z}$ calcd for $\mathrm{C}_{21} \mathrm{H}_{19} \mathrm{~N}_{4} \mathrm{O}[\mathrm{M}+\mathrm{H}]^{+} 343.1559$ found 343.1570 .

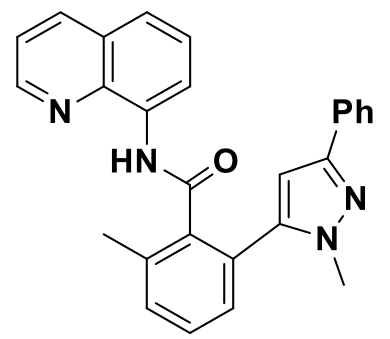

2-methyl-6-(1-methyl-3-phenyl-1H-pyrazol-5-yl)- $N$-(quinolin-8-yl)benzamide $\quad(3 \mathrm{~h})$ : The title compound was synthesized following General Procedure B. Light yellow solid in $70 \%(58.5 \mathrm{mg}, 0.14 \mathrm{mmol}), \mathrm{mp}=92-93{ }^{\circ} \mathrm{C} .{ }^{1} \mathrm{H} \mathrm{NMR}\left(400 \mathrm{MHz}, \mathrm{CDCl}_{3}\right): \delta 9.78(\mathrm{~s}$, $1 \mathrm{H}), 8.76(\mathrm{dd}, J=7.2,1.6 \mathrm{~Hz}, 1 \mathrm{H}), 8.67(\mathrm{dd}, J=4.3,1.6 \mathrm{~Hz}, 1 \mathrm{H}), 8.06(\mathrm{dt}, J=8.3,1.6$ Hz, 1H), $7.55-7.37$ (m, 6H), 7.34 (ddd, $J=8.2,4.3,1.4 \mathrm{~Hz}, 1 \mathrm{H}), 7.31-7.14(\mathrm{~m}, 4 \mathrm{H})$, $6.68(\mathrm{~d}, J=1.5 \mathrm{~Hz}, 1 \mathrm{H}), 3.86(\mathrm{~s}, 3 \mathrm{H}), 2.57(\mathrm{~s}, 3 \mathrm{H}) .{ }^{13} \mathrm{C}\left\{{ }^{1} \mathrm{H}\right\} \mathrm{NMR}\left(100 \mathrm{MHz}, \mathrm{CDCl}_{3}\right): \delta$ 
$167.15,150.49,148.17,142.38,138.42,138.39,136.42,134.15,133.46,131.44,129.32$, $128.44,128.43,128.33,128.10,128.02,127.41,127.40,125.53,122.15,121.64,116.81$, 104.92, 37.43, 19.88. FTIR (ATR, $\mathrm{cm}^{-1}$ ): 3337, 3134, 2929, 1672, 1520, 1483, 1424, 1325, 906, 723. HRMS (ESI-MS) $\mathrm{m} / \mathrm{z}$ calcd for $\mathrm{C}_{27} \mathrm{H}_{23} \mathrm{~N}_{4} \mathrm{O}[\mathrm{M}+\mathrm{H}]^{+} 419.1872$ found 419.1889.<smiles>Cc1cccc(-c2nn(-c3ccccc3)nc2C)c1C(=O)Nc1cccc2cccnc12</smiles>

\section{2-methyl-6-(5-methyl-2-phenyl-2H-1,2,3-triazol-4-yl)- $N$-(quinolin-8-yl)benzamide}

(3i): The title compound was synthesized following General Procedure A. Yellow solid in $40 \%$ (33.5 mg, $0.08 \mathrm{mmol}), \mathrm{mp}=140-142{ }^{\circ} \mathrm{C} .{ }^{1} \mathrm{H} \mathrm{NMR}\left(400 \mathrm{MHz}, \mathrm{CDCl}_{3}\right): \delta 9.95$ (s, $1 \mathrm{H}), 8.85(\mathrm{dd}, J=7.3,1.7 \mathrm{~Hz}, 1 \mathrm{H}), 8.60(\mathrm{dd}, J=4.2,1.7 \mathrm{~Hz}, 1 \mathrm{H}), 8.09$ (dd, $J=8.3$, $1.7 \mathrm{~Hz}, 1 \mathrm{H}), 7.67-7.60(\mathrm{~m}, 2 \mathrm{H}), 7.54-7.41(\mathrm{~m}, 4 \mathrm{H}), 7.41-7.36(\mathrm{~m}, 1 \mathrm{H}), 7.33$ (dd, $J=$ 8.3, 4.2 Hz, 1H), $7.15-7.08$ (m, 3H), 2.60 (s, 3H), 2.44 (s, 3H). ${ }^{13} \mathrm{C}\left\{{ }^{1} \mathrm{H}\right\}$ NMR (100 $\left.\mathrm{MHz}, \mathrm{CDCl}_{3}\right): \delta 167.98,148.26,145.67,143.89,139.56,138.59,137.65,136.70,136.22$, $134.59,131.05,129.31,128.85,128.16,128.00,127.41,126.70,121.84,121.58,118.26$, 116.86, 19.98, 11.21. FTIR (ATR, $\mathrm{cm}^{-1}$ ): 3344, 3059, 2923 1673, 1595, 1519, 1482, 1325, 907, 729. HRMS (ESI-MS) $\mathrm{m} / \mathrm{z}$ calcd for $\mathrm{C}_{26} \mathrm{H}_{22} \mathrm{~N}_{5} \mathrm{O}[\mathrm{M}+\mathrm{H}]^{+} 420.1824$ found 420.1839 .<smiles>Cc1cccc(-c2sc3ccccc3c2C)c1C(=O)Nc1cccc2cccnc12</smiles>

2-methyl-6-(3-methyl-1-benzothiophen-2-yl)- $N$-(quinolin-8-yl)benzamide (3j): The title compound was synthesized following General Procedure B. Light yellow solid in 70 
\% ( $57.1 \mathrm{mg}, 0.14 \mathrm{mmol}), \mathrm{mp}=211-212{ }^{\circ} \mathrm{C} .{ }^{1} \mathrm{H}$ NMR $\left(400 \mathrm{MHz}, \mathrm{CDCl}_{3}\right): \delta 9.81(\mathrm{~s}$, $1 \mathrm{H}), 8.75(\mathrm{dd}, J=7.4,1.6 \mathrm{~Hz}, 1 \mathrm{H}), 8.63(\mathrm{dd}, J=4.2,1.7 \mathrm{~Hz}, 1 \mathrm{H}), 8.02(\mathrm{dd}, J=8.3,1.7$ $\mathrm{Hz}, 1 \mathrm{H}), 7.62$ (dt, $J=7.9,0.9 \mathrm{~Hz}, 1 \mathrm{H}), 7.56(\mathrm{dt}, J=8.2,0.9 \mathrm{~Hz}, 1 \mathrm{H}), 7.47-7.40(\mathrm{~m}$, $3 \mathrm{H}), 7.39-7.36(\mathrm{~m}, 1 \mathrm{H}), 7.36-7.30(\mathrm{~m}, 2 \mathrm{H}), 7.27-7.21(\mathrm{~m}, 1 \mathrm{H}), 7.17(\mathrm{ddd}, J=8.3$, 7.0, $1.3 \mathrm{~Hz}, 1 \mathrm{H}), 2.56(\mathrm{~s}, 3 \mathrm{H}), 2.38(\mathrm{~s}, 3 \mathrm{H}) .{ }^{13} \mathrm{C}\left\{{ }^{1} \mathrm{H}\right\} \mathrm{NMR}\left(100 \mathrm{MHz}, \mathrm{CDCl}_{3}\right): \delta 167.42$, $147.97,140.35,139.67,138.72$, 138.42, 136.23, 136.19, 135.60, 134.45, 132.05, 130.87, 130.03, 129.46, 129.06, 127.89, 127.34, 124.14, 123.88, 122.06, 122.05, 121.78, 121.51, 116.64, 20.03, 12.76. FTIR (ATR, $\mathrm{cm}^{-1}$ ): 3340, 3061, 2919, 1671, 1520, 1482, 1325, 905, 727. HRMS (ESI-MS) m/z calcd for $\mathrm{C}_{26} \mathrm{H}_{21} \mathrm{~N}_{2} \mathrm{OS}[\mathrm{M}+\mathrm{H}]^{+} 409.1375$ found 409.1383.

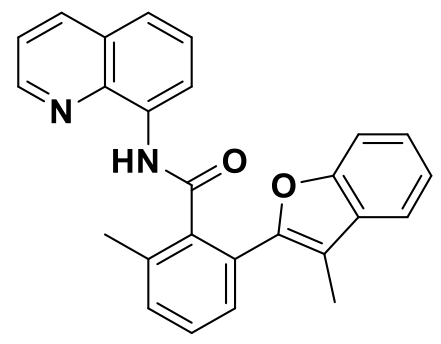

2-methyl-6-(3-methyl-1-benzofuran-2-yl)- $N$-(quinolin-8-yl)benzamide (3k): The title compound was synthesized following General Procedure B. Light yellow solid in $36 \%$ (28.2 mg, $0.07 \mathrm{mmol}), \mathrm{mp}=135-137{ }^{\circ} \mathrm{C} .{ }^{1} \mathrm{H}$ NMR $\left(400 \mathrm{MHz}, \mathrm{CDCl}_{3}\right): \delta 9.87(\mathrm{~s}, 1 \mathrm{H})$, $8.83(\mathrm{dd}, J=7.6,1.0 \mathrm{~Hz}, 1 \mathrm{H}), 8.60(\mathrm{dd}, J=4.3,1.7,0.7 \mathrm{~Hz}, 1 \mathrm{H}), 8.09-8.01(\mathrm{~m}, 1 \mathrm{H})$, $7.55-7.41(\mathrm{~m}, 4 \mathrm{H}), 7.41-7.28(\mathrm{~m}, 3 \mathrm{H}), 7.08-6.97(\mathrm{~m}, 3 \mathrm{H}), 2.60(\mathrm{~s}, 3 \mathrm{H}), 2.36(\mathrm{~s}, 3 \mathrm{H})$. ${ }^{13} \mathrm{C}\left\{{ }^{1} \mathrm{H}\right\}$ NMR $\left(100 \mathrm{MHz}, \mathrm{CDCl}_{3}\right): \delta 167.90,154.28,149.95,148.03,138.50,137.35$, $136.68,136.15,134.72$, 131.14, 130.38, 129.21, 128.68, 127.91, 127.70, 127.36, 124.17, 122.11, 121.73, 121.49, 119.28, 116.65, 113.51, 110.86, 20.00, 9.16. FTIR (ATR, $\left.\mathrm{cm}^{-1}\right)$ : 3342, 3052, 2986, 1673, 1519, 1482, 1384, 1325, 1079, 1263, 905, 728. HRMS (ESIMS) $\mathrm{m} / \mathrm{z}$ calcd for $\mathrm{C}_{26} \mathrm{H}_{21} \mathrm{~N}_{2} \mathrm{O}_{2}[\mathrm{M}+\mathrm{H}]^{+} 393.1603$ found 393.1618 . 
<smiles>Cc1cccc(-c2ccncc2F)c1C(=O)Nc1cccc2cccnc12</smiles>

2-(3-fluoropyridin-4-yl)-6-methyl- $N$-(quinolin-8-yl)benzamide (3I): $\quad$ The title compound was synthesized following General Procedure B. Yellow solid in $35 \%(25.0$ $\mathrm{mg}, 0.07 \mathrm{mmol}), \mathrm{mp}=154-155^{\circ} \mathrm{C} .{ }^{1} \mathrm{H} \mathrm{NMR}\left(400 \mathrm{MHz}, \mathrm{CDCl}_{3}\right): \delta 9.78(\mathrm{~s}, 1 \mathrm{H}), 8.72-$ $8.65(\mathrm{~m}, 2 \mathrm{H}), 8.34(\mathrm{~d}, J=1.7 \mathrm{~Hz}, 1 \mathrm{H}), 8.25(\mathrm{dd}, J=4.9,0.9 \mathrm{~Hz}, 1 \mathrm{H}), 8.11(\mathrm{dd}, J=8.3$, $1.7 \mathrm{~Hz}, 1 \mathrm{H}), 7.51-7.48(\mathrm{~m}, 2 \mathrm{H}), 7.46$ (d, J=7.6 Hz, 1H), $7.43-7.37$ (m, 3H), 7.32 $7.28(\mathrm{~m}, 1 \mathrm{H}), 2.57(\mathrm{~s}, 3 \mathrm{H}) .{ }^{13} \mathrm{C}\left\{{ }^{1} \mathrm{H}\right\} \mathrm{NMR}\left(100 \mathrm{MHz}, \mathrm{CDCl}_{3}\right): \delta 166.95,148.30,145.49$, $145.44,138.61,138.46,138.36,137.35$, 136.52, 136.40, 135.74, 135.61, 134.04, 131.54, 130.79, 129.44, 127.98, 127.91, 127.89, 127.35, 125.53, 122.26, 121.80, 116.75, 20.03. ${ }^{19} \mathrm{~F}$ NMR $\left(376 \mathrm{MHz}, \mathrm{CDCl}_{3}\right) \delta-130.14(\mathrm{~d}, J=6.5 \mathrm{~Hz})$. FTIR $\left(\mathrm{ATR}, \mathrm{cm}^{-1}\right): 3333,3132$, $1675,1523,1484,1424,1326,827,722$. HRMS (ESI-MS) $\mathrm{m} / \mathrm{z}$ calcd for $\mathrm{C}_{22} \mathrm{H}_{17} \mathrm{FN}_{3} \mathrm{O}[\mathrm{M}$ $+\mathrm{H}]^{+} 358.1356$ found 358.1370 .<smiles>Cc1cccc(-c2ccccc2F)c1C(=O)Nc1cccc2cccnc12</smiles>

2'-fluoro-3-methyl- $N$-(quinolin-8-yl)-[1,1'-biphenyl]-2-carboxamide (3r): The title compound was synthesized following General Procedure A. White solid in $70 \%$ (49.8 $\mathrm{mg}, 0.14 \mathrm{mmol}), \mathrm{mp}=78-80{ }^{\circ} \mathrm{C} .{ }^{1} \mathrm{H} \mathrm{NMR}\left(400 \mathrm{MHz}, \mathrm{CDCl}_{3}\right): \delta 9.77$ (s, 1H), 8.72 (dd, $J=6.8,2.2 \mathrm{~Hz}, 1 \mathrm{H}), 8.67(\mathrm{dd}, J=4.2,1.7 \mathrm{~Hz}, 1 \mathrm{H}), 8.09(\mathrm{dd}, J=8.3,1.7 \mathrm{~Hz}, 1 \mathrm{H}), 7.53-$ $7.36(\mathrm{~m}, 5 \mathrm{H}), 7.34(\mathrm{dd}, J=7.6,1.3 \mathrm{~Hz}, 1 \mathrm{H}), 7.30(\mathrm{dd}, J=7.6,1.4 \mathrm{~Hz}, 1 \mathrm{H}), 7.12-7.04$ (m, 1H), 6.99 (td, $J=7.5,1.3 \mathrm{~Hz}, 1 \mathrm{H}), 6.93(\mathrm{ddd}, J=9.7,8.1,1.3 \mathrm{~Hz}, 1 \mathrm{H}), 2.55(\mathrm{~s}, 3 \mathrm{H})$. 
${ }^{13} \mathrm{C}\left\{{ }^{1} \mathrm{H}\right\}$ NMR (100 MHz, $\left.\mathrm{CDCl}_{3}\right): \delta 167.73,159.75$ (d), 148.17, 138.52, 137.77, 136.19, 136.01, 134.45, 133.54, 131.60 (d), 130.35, 129.48 (d), 129.08, 128.47, 128.46, 127.89, 127.73, 127.36, 123.91 (d), 121.71 (d), 116.54, 115.60 (d), 20.04. ${ }^{19}$ F NMR $(376 \mathrm{MHz}$, $\left.\mathrm{CDCl}_{3}\right) \delta-115.43\left(\mathrm{td}, J=8.7,5.3 \mathrm{~Hz}\right.$ ). FTIR $\left(\right.$ ATR, $\mathrm{cm}^{-1}$ ): 3337, 2925, 1673, 1519, 1482, $1423,1325,1233,1026,826,753$. HRMS (ESI-MS) $\mathrm{m} / \mathrm{z}$ calcd for $\mathrm{C}_{23} \mathrm{H}_{18} \mathrm{FN}_{2} \mathrm{O}[\mathrm{M}+\mathrm{H}]^{+}$ 357.1403 found 357.1407 .

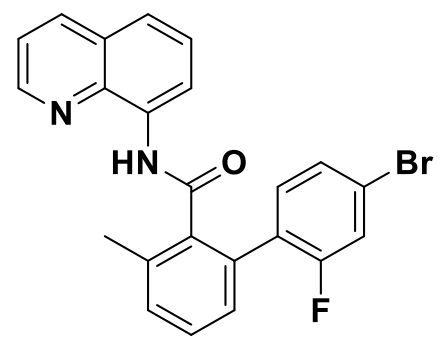

4'-bromo-2'-fluoro-3-methyl- $N$-(quinolin-8-yl)-[1,1'-biphenyl]-2-carboxamide (3s): The title compound was synthesized following General Procedure A. White solid in $58 \%$ (50.4 mg, $0.11 \mathrm{mmol}), \mathrm{mp}=67-68{ }^{\circ} \mathrm{C} .{ }^{1} \mathrm{H}$ NMR $\left(400 \mathrm{MHz}, \mathrm{CDCl}_{3}\right): \delta 9.78(\mathrm{~s}, 1 \mathrm{H})$, $8.73(\mathrm{dd}, J=6.0,3.0 \mathrm{~Hz}, 1 \mathrm{H}), 8.68(\mathrm{dd}, J=4.2,1.7 \mathrm{~Hz}, 1 \mathrm{H}), 8.12(\mathrm{dd}, J=8.3,1.7 \mathrm{~Hz}$, 1H), $7.54-7.48(\mathrm{~m}, 2 \mathrm{H}), 7.45-7.38(\mathrm{~m}, 2 \mathrm{H}), 7.35$ (ddd, $J=7.6,1.4,0.7 \mathrm{~Hz}, 1 \mathrm{H}), 7.31$ $(\mathrm{d}, J=7.8 \mathrm{~Hz}, 1 \mathrm{H}), 7.27-7.23(\mathrm{~m}, 1 \mathrm{H}), 7.19-7.10(\mathrm{~m}, 2 \mathrm{H}), 2.55$ (s, 3H). ${ }^{13} \mathrm{C}\left\{{ }^{1} \mathrm{H}\right\}$ NMR (100 MHz, $\left.\mathrm{CDCl}_{3}\right): \delta 167.47,159.54$ (d), 148.24, 138.51, 137.63, 136.34, 136.12, $134.26,132.61$ (d), 132.44, 130.70, 129.20, 128.33, 127.98, 127.38, 127.31 (d), 127.01 (d), 122.06, 121.91 (d), 121.73, 119.32 (d), 116.75, 20.04. ${ }^{19} \mathrm{~F} \mathrm{NMR}\left(376 \mathrm{MHz}, \mathrm{CDCl}_{3}\right) \delta$ -112.36 (t, $J=8.6 \mathrm{~Hz}$ ). FTIR (ATR, $\mathrm{cm}^{-1}$ ): 3343, 3064, 1675, 1523, 1483, 1424, 1386, 1326, 892, 790. HRMS (ESI-MS) $\mathrm{m} / \mathrm{z}$ calcd for $\mathrm{C}_{23} \mathrm{H}_{17} \mathrm{BrFN}_{2} \mathrm{O}[\mathrm{M}+\mathrm{H}]^{+} 435.0508$ found 435.0505 . 
<smiles>Cc1cccc(-c2ccc(F)cc2F)c1C(=O)Nc1cccc2cccnc12</smiles>

2',4'-difluoro-3-methyl- $N$-(quinolin-8-yl)-[1,1'-biphenyl]-2-carboxamide $\quad(3 t):$ The title compound was synthesized following General Procedure A. White solid in $55 \%$ (41.1 mg, $0.10 \mathrm{mmol}) . \mathrm{mp}=136-138{ }^{\circ} \mathrm{C} .{ }^{1} \mathrm{H} \mathrm{NMR}\left(400 \mathrm{MHz}, \mathrm{CDCl}_{3}\right): \delta 9.77(\mathrm{~s}, 1 \mathrm{H})$, $8.72(\mathrm{dd}, J=6.1,2.9 \mathrm{~Hz}, 1 \mathrm{H}), 8.68(\mathrm{dd}, J=4.2,1.7 \mathrm{~Hz}, 1 \mathrm{H}), 8.12(\mathrm{dd}, J=8.3,1.7 \mathrm{~Hz}$, 1H), $7.54-7.47(\mathrm{~m}, 2 \mathrm{H}), 7.45-7.37(\mathrm{~m}, 3 \mathrm{H}), 7.37-7.32(\mathrm{~m}, 1 \mathrm{H}), 7.29-7.22(\mathrm{~m}, 1 \mathrm{H})$, $6.79-6.65(\mathrm{~m}, 2 \mathrm{H}), 2.54(\mathrm{~s}, 3 \mathrm{H}) .{ }^{13} \mathrm{C}\left\{{ }^{1} \mathrm{H}\right\} \mathrm{NMR}\left(100 \mathrm{MHz}, \mathrm{CDCl}_{3}\right): \delta 167.61,162.24$ (dd), 159.42 (dd), 148.20, 138.48, 137.89, 136.36, 136.07, 134.29, 132.61, 132.33 (dd), 130.53, 129.16, 128.50, 127.97, 127.40, 123.97 (dd), 121.99, 121.72, 116.67, 111.18(dd), 104.01 (dd), 20.01. ${ }^{19} \mathrm{~F}$ NMR (376 MHz, $\left.\mathrm{CDCl}_{3}\right) \delta-110.75--110.93(\mathrm{~m})$. FTIR (ATR, $\mathrm{cm}^{-1}$ ): 3342, 3059, 1673, 1519, 1482, 1423, 1385, 1326, 1266, 966, 791. HRMS (ESIMS) $\mathrm{m} / \mathrm{z}$ calcd for $\mathrm{C}_{23} \mathrm{H}_{17} \mathrm{~F}_{2} \mathrm{~N}_{2} \mathrm{O}[\mathrm{M}+\mathrm{H}]^{+} 375.1309$ found 375.1318 .<smiles>Cc1ccc(F)c(-c2cccc(C)c2C(=O)Nc2cccc3cccnc23)c1</smiles>

2'-fluoro-3,5'-dimethyl- $N$-(quinolin-8-yl)-[1,1'-biphenyl]-2-carboxamide (3u): The title compound was synthesized following General Procedure A. White solid in $60 \%$ (44.4 mg, $0.12 \mathrm{mmol}), \mathrm{mp}=132-134{ }^{\circ} \mathrm{C} .{ }^{1} \mathrm{H} \mathrm{NMR}\left(400 \mathrm{MHz}, \mathrm{CDCl}_{3}\right) \delta 9.78(\mathrm{~s}, 1 \mathrm{H})$, $8.74(\mathrm{dd}, J=7.0,1.9 \mathrm{~Hz}, 1 \mathrm{H}), 8.68$ (dd, $J=4.2,1.7 \mathrm{~Hz}, 1 \mathrm{H}), 8.09$ (dd, $J=8.3,1.7 \mathrm{~Hz}$, 1H), $7.52-7.44(\mathrm{~m}, 2 \mathrm{H}), 7.44-7.36(\mathrm{~m}, 2 \mathrm{H}), 7.32(\mathrm{dt}, J=7.6,1.0 \mathrm{~Hz}, 1 \mathrm{H}), 7.30-7.25$ (m, 1H), $7.23(\mathrm{dd}, J=7.3,2.2 \mathrm{~Hz}, 1 \mathrm{H}), 6.86-6.74(\mathrm{~m}, 2 \mathrm{H}), 2.55(\mathrm{~s}, 3 \mathrm{H}), 2.14$ (s, $3 \mathrm{H}) .{ }^{13} \mathrm{C}\left\{{ }^{1} \mathrm{H}\right\}$ NMR $\left(100 \mathrm{MHz}, \mathrm{CDCl}_{3}\right): \delta 167.81,157.91$ (d), 148.11, 138.51, 137.72, 
136.22, 135.96, 134.52, 133.80, 133.22 (d), 131.95 (d), 130.25, 129.87, 129.79, 129.08, $128.41,127.90,127.37,127.35$ (d), 121.66 (d), 116.49, 115.18 (d), 20.57, 20.05. ${ }^{19} \mathrm{~F}$ NMR (376 MHz, $\left.\mathrm{CDCl}_{3}\right) \delta-120.81(\mathrm{q}, J=7.3 \mathrm{~Hz})$. FTIR (ATR, $\mathrm{cm}^{-1}$ ): 3344, 3061, 1677, $1522,1483,1424,1385,1326,826,760$. HRMS (ESI-MS) $\mathrm{m} / \mathrm{z}$ calcd for $\mathrm{C}_{24} \mathrm{H}_{20} \mathrm{FN}_{2} \mathrm{O}$ [M $+\mathrm{H}]^{+} 371.1560$ found 371.1572 .<smiles>Cc1cccc(-c2ccccc2Cl)c1C(=O)Nc1cccc2cccnc12</smiles>

2'-chloro-3-methyl- $N$-(quinolin-8-yl)-[1,1'-biphenyl]-2-carboxamide (3v): The title compound was synthesized following General Procedure A. White solid in $42 \%$ (31.3 $\mathrm{mg}, 0.08 \mathrm{mmol}), \mathrm{mp}=181-183{ }^{\circ} \mathrm{C} .{ }^{1} \mathrm{H}$ NMR $\left(400 \mathrm{MHz}, \mathrm{CDCl}_{3}\right) \delta 9.78(\mathrm{~s}, 1 \mathrm{H}), 8.70$ $(\mathrm{dd}, J=4.1,1.9 \mathrm{~Hz}, 1 \mathrm{H}), 8.68-8.62(\mathrm{~m}, 1 \mathrm{H}), 8.08(\mathrm{dq}, J=8.4,1.4 \mathrm{~Hz}, 1 \mathrm{H}), 7.43(\mathrm{td}, J=$ $5.1,1.5 \mathrm{~Hz}, 2 \mathrm{H}), 7.41-7.35(\mathrm{~m}, 3 \mathrm{H}), 7.32(\mathrm{~d}, J=7.7 \mathrm{~Hz}, 1 \mathrm{H}), 7.26-7.20(\mathrm{~m}, 2 \mathrm{H}), 7.13$ $-7.06(\mathrm{~m}, 1 \mathrm{H}), 7.05-6.98(\mathrm{~m}, 1 \mathrm{H}), 2.52(\mathrm{~s}, 3 \mathrm{H}) .{ }^{13} \mathrm{C}\left\{{ }^{1} \mathrm{H}\right\}$ NMR $\left(100 \mathrm{MHz}, \mathrm{CDCl}_{3}\right) \delta$ $167.55,148.07,138.89,138.46,137.49,137.03,136.16,135.83,134.43,133.27,131.43$, 130.21, 129.46, 128.96, 128.81, 128.17, 127.86, 127.32, 126.44, 121.75, 121.66, 116.46, 19.95. FTIR (ATR, $\mathrm{cm}^{-1}$ ): 3339, 3127, 2915, 1676, 1522, 1483, 1425, 1326, 900, 730. HRMS (ESI-MS) $\mathrm{m} / \mathrm{z}$ calcd for $\mathrm{C}_{23} \mathrm{H}_{18} \mathrm{ClN}_{2} \mathrm{O}[\mathrm{M}+\mathrm{H}]^{+} 373.1108$ found 373.1113 .<smiles>COc1ccc(Cl)c(-c2cccc(C)c2C(=O)Nc2cccc3cccnc23)c1</smiles>

2'-chloro-5'-methoxy-3-methyl- $N$-(quinolin-8-yl)-[1,1'-biphenyl]-2-carboxamide (3w): The title compound was synthesized following General Procedure A. Yellow solid 
in $40 \%(32.2 \mathrm{mg}, 0.08 \mathrm{mmol}), \mathrm{mp}=52-53{ }^{\circ} \mathrm{C} .{ }^{1} \mathrm{H}$ NMR $\left(400 \mathrm{MHz}, \mathrm{CDCl}_{3}\right): \delta 9.81(\mathrm{~s}$, 1H), $8.73-8.68(\mathrm{~m}, 2 \mathrm{H}), 8.08(\mathrm{dd}, J=8.3,1.7 \mathrm{~Hz}, 1 \mathrm{H}), 7.49-7.43(\mathrm{~m}, 2 \mathrm{H}), 7.42-7.36$ (m, 2H), 7.34 (ddd, $J=7.7,1.4,0.7 \mathrm{~Hz}, 1 \mathrm{H}), 7.27-7.22(\mathrm{~m}, 1 \mathrm{H}), 7.11(\mathrm{~d}, J=8.8 \mathrm{~Hz}$, $1 \mathrm{H}), 6.96(\mathrm{~d}, J=3.0 \mathrm{~Hz}, 1 \mathrm{H}), 6.57(\mathrm{dd}, J=8.8,3.1 \mathrm{~Hz}, 1 \mathrm{H}), 3.64(\mathrm{~s}, 3 \mathrm{H}), 2.55$ (s, 3H). ${ }^{13} \mathrm{C}\left\{{ }^{1} \mathrm{H}\right\}$ NMR $\left(100 \mathrm{MHz}, \mathrm{CDCl}_{3}\right): \delta 167.55,157.79,148.03,139.57,138.43,137.35$, $137.08,136.17,135.90,134.48,130.27,130.13,128.85,128.06,127.86,127.32$, 124.58, 121.77, 121.64, 116.43, 115.94, 115.73, 55.61, 19.94. FTIR (ATR, $\mathrm{cm}^{-1}$ ): 3333, 3063, 2958, 1671, 1519, 1482, 1423, 1324, 1228, 905, 728. HRMS (ESI-MS) m/z calcd for $\mathrm{C}_{24} \mathrm{H}_{20} \mathrm{ClN}_{2} \mathrm{O}_{2}[\mathrm{M}+\mathrm{H}]^{+} 403.1213$ found 403.1226 .

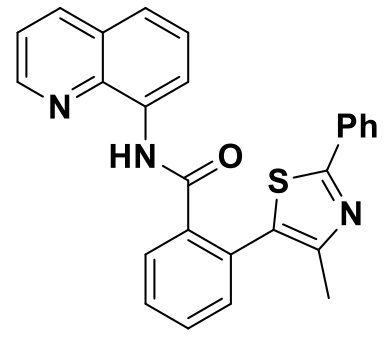

2-(4-methyl-2-phenyl-1,3-thiazol-5-yl)- $N$-(quinolin-8-yl)benzamide $\quad(3 z):$ The title compound was synthesized following General Procedure A. Yellow solid in $45 \%$ (37.9 $\mathrm{mg}, 0.09 \mathrm{mmol}), \mathrm{mp}=138-140{ }^{\circ} \mathrm{C} .{ }^{1} \mathrm{H} \mathrm{NMR}\left(400 \mathrm{MHz}, \mathrm{CDCl}_{3}\right): \delta 10.20(\mathrm{~s}, 1 \mathrm{H}), 8.85$ $(\mathrm{dd}, J=7.6,1.4 \mathrm{~Hz}, 1 \mathrm{H}), 8.49$ (dd, $J=4.2,1.7 \mathrm{~Hz}, 1 \mathrm{H}), 8.11-8.05$ (m, 1H), 8.03 (dd, $J$ $=8.3,1.7 \mathrm{~Hz}, 1 \mathrm{H}), 7.85-7.76(\mathrm{~m}, 2 \mathrm{H}), 7.62-7.55(\mathrm{~m}, 2 \mathrm{H}), 7.55-7.48(\mathrm{~m}, 2 \mathrm{H}), 7.45$ $(\mathrm{dd}, J=8.3,1.4 \mathrm{~Hz}, 1 \mathrm{H}), 7.36(\mathrm{ddd}, J=4.9,2.9,1.6 \mathrm{~Hz}, 3 \mathrm{H}), 7.27-7.21(\mathrm{~m}, 1 \mathrm{H}), 2.41$ (s, 3H). ${ }^{13} \mathrm{C}\left\{{ }^{1} \mathrm{H}\right\}$ NMR $\left(100 \mathrm{MHz}, \mathrm{CDCl}_{3}\right): \delta 166.87,166.32,151.47,148.04,138.58$, $137.11,136.14,134.60,133.66,132.54,130.88,130.17,129.98,129.59,129.24,129.17$, 128.94, 127.92, 127.38, 126.40, 121.90, 121.56, 116.52, 15.93. FTIR (ATR, $\mathrm{cm}^{-1}$ ): 3315, 2927, 1665, 1524, 1484, 1423, 1325, 755, 688. HRMS (ESI-MS) m/z calcd for $\mathrm{C}_{26} \mathrm{H}_{20} \mathrm{~N}_{3} \mathrm{OS}[\mathrm{M}+\mathrm{H}]^{+} 422.1327$ found 422.1342 . 
<smiles>Cc1nc(-c2ccccc2)sc1-c1cccc(-c2sc(-c3ccccc3)nc2C)c1C(=O)Nc1cccc2ccccc12</smiles>

2,6-bis(4-methyl-2-phenyl-1,3-thiazol-5-yl)- $N$-(quinolin-8-yl)benzamide (3z'): The title compound was synthesized following General Procedure A. Yellow solid in $26 \%$ (30.8 mg, $0.05 \mathrm{mmol}), \mathrm{mp}=155-157{ }^{\circ} \mathrm{C} .{ }^{1} \mathrm{H} \mathrm{NMR}\left(400 \mathrm{MHz}, \mathrm{CDCl}_{3}\right): \delta 9.81(\mathrm{~s}, 1 \mathrm{H})$, $8.64(\mathrm{dd}, J=4.2,1.7 \mathrm{~Hz}, 1 \mathrm{H}), 8.56(\mathrm{dd}, J=5.0,4.0 \mathrm{~Hz}, 1 \mathrm{H}), 8.02(\mathrm{dd}, J=8.3,1.7 \mathrm{~Hz}$, 1H), $7.81-7.73(\mathrm{~m}, 4 \mathrm{H}), 7.61(\mathrm{dd}, J=8.9,6.1 \mathrm{~Hz}, 1 \mathrm{H}), 7.58-7.53(\mathrm{~m}, 2 \mathrm{H}), 7.41-7.37$ $(\mathrm{m}, 2 \mathrm{H}), 7.37-7.28(\mathrm{~m}, 7 \mathrm{H}), 2.48(\mathrm{~s}, 6 \mathrm{H}) .{ }^{13} \mathrm{C}\left\{{ }^{1} \mathrm{H}\right\} \mathrm{NMR}\left(100 \mathrm{MHz}, \mathrm{CDCl}_{3}\right): \delta 166.70$, $165.50,151.35,148.05,139.88,138.31$, 136.30, 133.99, 133.52, 132.37, 130.70, 129.92, $129.45,128.87,128.48,127.90,127.36,126.45,122.07,121.58,116.86,16.19$. FTIR $\left(\mathrm{ATR}, \mathrm{cm}^{-1}\right): 3329,3056,1672,1521,1484,1424,1384,1326,905,726$. HRMS (ESIMS) $\mathrm{m} / \mathrm{z}$ calcd for $\mathrm{C}_{36} \mathrm{H}_{27} \mathrm{~N}_{4} \mathrm{OS}_{2}[\mathrm{M}+\mathrm{H}]^{+} 595.1626$ found 595.1646.<smiles>Cc1ccc(-c2sc(-c3ccccc3)nc2C)c(C(=O)Nc2cccc3cccnc23)c1</smiles>

5-methyl-2-(4-methyl-2-phenyl-1,3-thiazol-5-yl)- $N$-(quinolin-8-yl)benzamide (3aa): The title compound was synthesized following General Procedure A. Yellow solid in 86 $\%$ (74.9 mg, $0.17 \mathrm{mmol}), \mathrm{mp}=147-148{ }^{\circ} \mathrm{C} .{ }^{1} \mathrm{H} \mathrm{NMR}\left(400 \mathrm{MHz}, \mathrm{CDCl}_{3}\right): \delta 10.18(\mathrm{~s}$, $1 \mathrm{H}), 8.85(\mathrm{dd}, J=7.6,1.4 \mathrm{~Hz}, 1 \mathrm{H}), 8.48(\mathrm{dd}, J=4.2,1.7 \mathrm{~Hz}, 1 \mathrm{H}), 8.01(\mathrm{dd}, J=8.3,1.7$ $\mathrm{Hz}, 1 \mathrm{H}), 7.90(\mathrm{q}, J=1.0 \mathrm{~Hz}, 1 \mathrm{H}), 7.85-7.76(\mathrm{~m}, 2 \mathrm{H}), 7.51$ (t, $J=7.9 \mathrm{~Hz}, 1 \mathrm{H}), 7.44$ (dd, $J=8.3,1.4 \mathrm{~Hz}, 1 \mathrm{H}), 7.40-7.31(\mathrm{~m}, 5 \mathrm{H}), 7.23(\mathrm{dd}, J=8.3,4.2 \mathrm{~Hz}, 1 \mathrm{H}), 2.49(\mathrm{~s}, 3 \mathrm{H})$, $2.40(\mathrm{~s}, 3 \mathrm{H}) .{ }^{13} \mathrm{C}\left\{{ }^{1} \mathrm{H}\right\} \mathrm{NMR}\left(100 \mathrm{MHz}, \mathrm{CDCl}_{3}\right): \delta 166.65,166.51,151.34,148.01$, $139.35,138.57,136.83,136.10,134.63$, 133.70, 132.45, 131.66, 130.73, 129.91, 129.33, 
$128.92,127.90,127.37,126.49,126.37,121.84,121.53,116.50,21.35,15.89$. FTIR $\left(\right.$ ATR, $\left.\mathrm{cm}^{-1}\right): 3316,3051,2926,1662,1522,1483,1424,1383,1325,906,698$. HRMS (ESI-MS) $\mathrm{m} / \mathrm{z}$ calcd for $\mathrm{C}_{27} \mathrm{H}_{22} \mathrm{~N}_{3} \mathrm{OS}[\mathrm{M}+\mathrm{H}]^{+} 436.1484$ found 436.1501 .

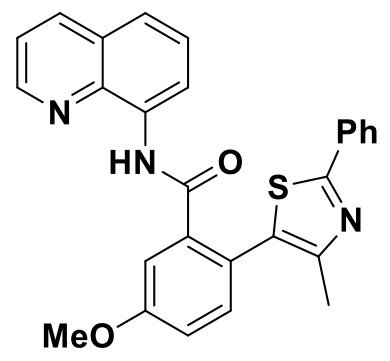

\section{5-methoxy-2-(4-methyl-2-phenyl-1,3-thiazol-5-yl)- $N$-(quinolin-8-yl)benzamide (3ab):}

The title compound was synthesized following General Procedure A. Light pink solid in $80 \%$ (72.1 mg, $0.16 \mathrm{mmol}), \mathrm{mp}=162-164{ }^{\circ} \mathrm{C} .{ }^{1} \mathrm{H}$ NMR $\left(400 \mathrm{MHz}, \mathrm{CDCl}_{3}\right): \delta 10.21$ (s, $1 \mathrm{H}), 8.83(\mathrm{dd}, J=7.6,1.4 \mathrm{~Hz}, 1 \mathrm{H}), 8.45(\mathrm{dd}, J=4.2,1.6 \mathrm{~Hz}, 1 \mathrm{H}), 7.98(\mathrm{dt}, J=8.4$, $1.8 \mathrm{~Hz}, 1 \mathrm{H}), 7.78(\mathrm{dd}, J=6.7,2.9 \mathrm{~Hz}, 2 \mathrm{H}), 7.61(\mathrm{~d}, J=2.7 \mathrm{~Hz}, 1 \mathrm{H}), 7.49(\mathrm{t}, J=7.9 \mathrm{~Hz}$, 1H), $7.45-7.36$ (m, 2H), $7.36-7.30(\mathrm{~m}, 3 \mathrm{H}), 7.20$ (ddd, $J=8.1,4.1,1.9 \mathrm{~Hz}, 1 \mathrm{H}), 7.13-$ 7.05 (m, 1H), 3.91 (s, 3H), $2.38(\mathrm{~s}, 3 \mathrm{H}) .{ }^{13} \mathrm{C}\left\{{ }^{1} \mathrm{H}\right\} \mathrm{NMR}\left(100 \mathrm{MHz}, \mathrm{CDCl}_{3}\right): \delta$ 166.56, 166.02, 160.17, 151.48, 148.03, 148.02, 138.59, 138.14, 136.07, 134.56, 133.88, 133.77, 129.86, 129.14, 128.91, 127.89, 127.33, 126.33, 121.92, 121.54, 121.46, 117.57, 116.51, 114.57, 114.55, 55.77, 15.88. FTIR (ATR, $\mathrm{cm}^{-1}$ ): 3317, 3052, 2960, 1661, 1523, 1483, $1424,1385,1326,1038,906,727$. HRMS (ESI-MS) m/z calcd for $\mathrm{C}_{27} \mathrm{H}_{22} \mathrm{~N}_{3} \mathrm{O}_{2} \mathrm{~S}[\mathrm{M}+$ $\mathrm{H}]^{+} 452.1433$ found 452.1449 .<smiles>Cc1nc(-c2ccccc2)sc1-c1ccc(Cl)cc1C(=O)Nc1cccc2cccnc12</smiles>

5-chloro-2-(4-methyl-2-phenyl-1,3-thiazol-5-yl)- $N$-(quinolin-8-yl)benzamide (3ac): The title compound was synthesized following General Procedure A. Yellow solid in 61 
$\%(55.6 \mathrm{mg}, 0.12 \mathrm{mmol}), \mathrm{mp}=166-168{ }^{\circ} \mathrm{C} .{ }^{1} \mathrm{H}$ NMR $\left(400 \mathrm{MHz}, \mathrm{CDCl}_{3}\right): \delta 10.21(\mathrm{~s}$, $1 \mathrm{H}), 8.82(\mathrm{dd}, J=7.5,1.5 \mathrm{~Hz}, 1 \mathrm{H}), 8.48(\mathrm{dd}, J=4.2,1.7 \mathrm{~Hz}, 1 \mathrm{H}), 8.09$ (d, $J=2.3 \mathrm{~Hz}$, $1 \mathrm{H}), 8.03(\mathrm{dd}, J=8.3,1.7 \mathrm{~Hz}, 1 \mathrm{H}), 7.84-7.76(\mathrm{~m}, 2 \mathrm{H}), 7.55(\mathrm{dd}, J=8.3,2.4 \mathrm{~Hz}, 1 \mathrm{H})$, $7.51(\mathrm{~d}, J=7.6 \mathrm{~Hz}, 1 \mathrm{H}), 7.49-7.42(\mathrm{~m}, 2 \mathrm{H}), 7.40-7.33(\mathrm{~m}, 3 \mathrm{H}), 7.28-7.22(\mathrm{~m}, 1 \mathrm{H})$, $2.39(\mathrm{~s}, 3 \mathrm{H}) .{ }^{13} \mathrm{C}\left\{{ }^{1} \mathrm{H}\right\}$ NMR $\left(100 \mathrm{MHz}, \mathrm{CDCl}_{3}\right): \delta 167.27,164.77,151.92,148.13$, 138.56, 138.39, 136.18, 135.47, 134.32, 133.84, 133.54, 130.99, 130.38, 130.14, 128.99, 128.00, 127.93, 127.92, 127.37, 126.43, 122.20, 121.64, 116.70, 15.95. FTIR (ATR, cm ${ }^{-}$ $\left.{ }^{1}\right):$ 3310, 3061, 2924, 1663, 1522, 1484, 1424, 1326, 908, 761, 728. HRMS (ESI-MS) m/z calcd for $\mathrm{C}_{26} \mathrm{H}_{19} \mathrm{ClN}_{3} \mathrm{OS}[\mathrm{M}+\mathrm{H}]^{+} 456.0937$ found 456.0955 .

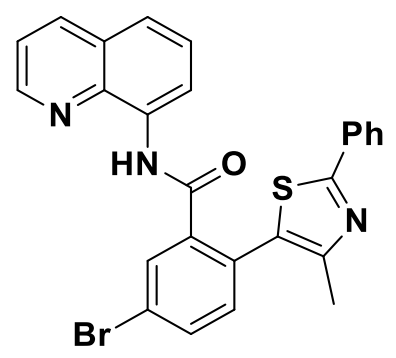

5-bromo-2-(4-methyl-2-phenyl-1,3-thiazol-5-yl)- $N$-(quinolin-8-yl)benzamide (3ad): The title compound was synthesized following General Procedure A. Yellow solid in 55 $\%(55.0 \mathrm{mg}, 0.11 \mathrm{mmol}), \mathrm{mp}=156-158{ }^{\circ} \mathrm{C} .{ }^{1} \mathrm{H} \mathrm{NMR}\left(400 \mathrm{MHz}, \mathrm{CDCl}_{3}\right): \delta 10.19(\mathrm{~s}$, $1 \mathrm{H}), 8.81(\mathrm{dd}, J=7.5,1.5 \mathrm{~Hz}, 1 \mathrm{H}), 8.47(\mathrm{dd}, J=4.2,1.7 \mathrm{~Hz}, 1 \mathrm{H}), 8.24(\mathrm{~d}, J=2.1 \mathrm{~Hz}$, $1 \mathrm{H}), 8.03(\mathrm{dd}, J=8.3,1.7 \mathrm{~Hz}, 1 \mathrm{H}), 7.84-7.76(\mathrm{~m}, 2 \mathrm{H}), 7.70(\mathrm{dd}, J=8.2,2.2 \mathrm{~Hz}, 1 \mathrm{H})$, $7.55-7.43(\mathrm{~m}, 2 \mathrm{H}), 7.40-7.33(\mathrm{~m}, 4 \mathrm{H}), 7.27-7.21(\mathrm{~m}, 1 \mathrm{H}), 2.39(\mathrm{~s}, 3 \mathrm{H}) .{ }^{13} \mathrm{C}\left\{{ }^{1} \mathrm{H}\right\}$ NMR $\left(100 \mathrm{MHz}, \mathrm{CDCl}_{3}\right): \delta 167.29,164.65,151.88,148.12,138.54,136.16,134.29$, $133.98,133.94,133.52,133.27,130.14,128.99,128.47,127.95,127.35,126.42,123.45$, 122.19, 121.63, 116.69, 15.95. FTIR (ATR, $\mathrm{cm}^{-1}$ ): 3308, 3065, 1662, 1523, 1484, 1424, 1326, 905, 824, 726. HRMS (ESI-MS) m/z calcd for $\mathrm{C}_{26} \mathrm{H}_{19} \mathrm{BrN}_{3} \mathrm{OS}[\mathrm{M}+\mathrm{H}]^{+} 500.0432$ found 500.0452 . 


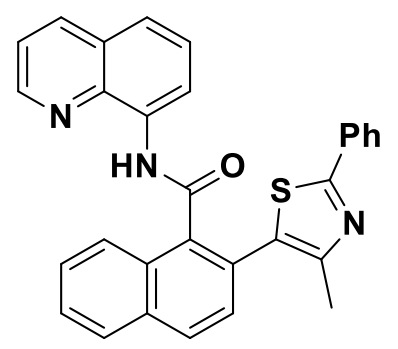

\section{2-(4-methyl-2-phenyl-1,3-thiazol-5-yl)- $N$-(quinolin-8-yl)naphthalene-1-carboxamide}

(3ae): The title compound was synthesized following General Procedure A. Light yellow solid in $80 \%$ (75.4 mg, $0.16 \mathrm{mmol}), \mathrm{mp}=98-100{ }^{\circ} \mathrm{C} .{ }^{1} \mathrm{H} \mathrm{NMR}\left(400 \mathrm{MHz}, \mathrm{CDCl}_{3}\right): \delta$ 10.04 (s, 1H), 8.94 (dd, $J=7.6,1.5 \mathrm{~Hz}, 1 \mathrm{H}), 8.61$ (dd, $J=4.2,1.7 \mathrm{~Hz}, 1 \mathrm{H}), 8.29-8.21$ (m, 1H), 8.07 (dd, $J=8.3,1.7 \mathrm{~Hz}, 1 \mathrm{H}), 8.04-7.99(\mathrm{~m}, 1 \mathrm{H}), 7.98-7.93(\mathrm{~m}, 1 \mathrm{H}), 7.80$ $7.72(\mathrm{~m}, 2 \mathrm{H}), 7.63-7.51(\mathrm{~m}, 4 \mathrm{H}), 7.48(\mathrm{dd}, J=8.3,1.5 \mathrm{~Hz}, 1 \mathrm{H}), 7.36-7.28(\mathrm{~m}, 4 \mathrm{H})$, $2.51(\mathrm{~s}, 3 \mathrm{H}) .{ }^{13} \mathrm{C}\left\{{ }^{1} \mathrm{H}\right\} \mathrm{NMR}\left(100 \mathrm{MHz}, \mathrm{CDCl}_{3}\right): \delta 166.78,166.77,151.42,148.17$, $138.44,136.41,136.29,134.41,133.54,133.32$, 130.49, 129.87, 129.85, 129.15, 128.83, $128.55,128.26,127.99,127.89,127.39$, 127.27, 126.93, 126.41, 125.92, 122.21, 121.68, 116.85, 16.23. HRMS (ESI-MS) $\mathrm{m} / \mathrm{z}$ calcd for $\mathrm{C}_{30} \mathrm{H}_{22} \mathrm{~N}_{3} \mathrm{OS}[\mathrm{M}+\mathrm{H}]^{+} 472.1484$ found 472.1504 .

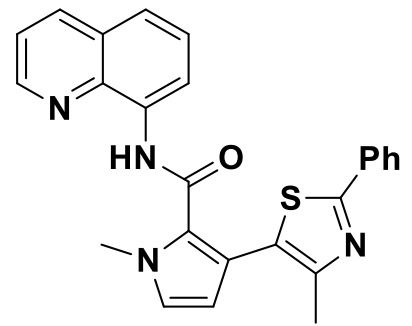

1-methyl-3-(4-methyl-2-phenyl-1,3-thiazol-5-yl)- $N$-(quinolin-8-yl)-1H-pyrrole-2-

carboxamide (3af): The title compound was synthesized following General Procedure A. Yellow solid in $74 \%(62.8 \mathrm{mg}, 0.15 \mathrm{mmol}), \mathrm{mp}=75-77^{\circ} \mathrm{C} .{ }^{1} \mathrm{H}$ NMR $(400 \mathrm{MHz}$, $\left.\mathrm{CDCl}_{3}\right): \delta 10.34(\mathrm{~s}, 1 \mathrm{H}), 8.83(\mathrm{dd}, J=7.7,1.3 \mathrm{~Hz}, 1 \mathrm{H}), 8.30(\mathrm{dd}, J=4.2,1.7 \mathrm{~Hz}, 1 \mathrm{H})$, $7.97(\mathrm{dd}, J=8.3,1.7 \mathrm{~Hz}, 1 \mathrm{H}), 7.95-7.88(\mathrm{~m}, 2 \mathrm{H}), 7.49(\mathrm{t}, J=8.0 \mathrm{~Hz}, 1 \mathrm{H}), 7.46-7.36$ (m, 4H), $7.15(\mathrm{dd}, J=8.3,4.2 \mathrm{~Hz}, 1 \mathrm{H}), 6.85(\mathrm{~d}, J=2.6 \mathrm{~Hz}, 1 \mathrm{H}), 6.27-6.22(\mathrm{~m}, 1 \mathrm{H})$, 4.12 (s, 3H), 2.37 (s, 3H). ${ }^{13} \mathrm{C}\left\{{ }^{1} \mathrm{H}\right\}$ NMR (100 MHz, $\left.\mathrm{CDCl}_{3}\right): \delta$ 166.73, 159.79, 152.40, $148.00,138.61,135.83,135.15,133.98,129.86,129.03,127.91,127.89$, 127.25, 126.38, 
124.94, 124.91, 121.50, 121.22, 117.17, 115.82, 111.50, 38.17, 15.92. FTIR (ATR, $\left.\mathrm{cm}^{-1}\right)$ : 3293, 2954, 1656, 1525, 1484, 1424, 1326, 824, 760. HRMS (ESI-MS) m/z calcd for $\mathrm{C}_{25} \mathrm{H}_{21} \mathrm{~N}_{4} \mathrm{OS}[\mathrm{M}+\mathrm{H}]^{+} 425.1436$ found 425.1454 . 
Chapter 3: A Nickel-Catalyzed Sequential Oxidative Decarboxylative (Hetero)Arylation and Cyclization for the Synthesis of Phenanthridinones

\subsection{Overview}

As our work in Chapter 2 shows, we were able to achieve a new nickel-catalyzed oxidative decarboxylative (hetero)arylation of unactivated $\mathrm{C}-\mathrm{H}$ bonds. During the optimization of the reaction conditions for the Ni-catalyzed ODC reaction, we noticed the formation of a small amount of byproduct, which we later characterized to be the phenanthridinone products. We found that phenanthridinones that contain heterocycles are limited under oxidative conditions as coupling partners. Knowing our new catalyst system works with heteroaromatic carboxylates, we should be able to achieve a sequential heteroarylation and cyclization to form heterocycle-containing phenanthridinones.

Phenanthridinones are key structures found in a variety of natural products and biologically active molecules ${ }^{51}$ such as, anticancer therapeutics, ${ }^{52}$ polymerase (PARP) ${ }^{53}$ aurora kinase inhibitors, ${ }^{54}$ antimalarial, ${ }^{55}$ anti-HIV, ${ }^{56}$ and antituberculosis activities ${ }^{57}$ to name a few. In addition, phenanthridinone derivatives are also utilized in material sciences because of the planar heterocycle-containing system providing effective photoconducting and photovoltaic properties in optoelectronics and electroluminescence (Figure 1). ${ }^{58}$

Heterocycle-containing phenanthridinones, however, are less explored than their nonheterocycle-containing counterparts. The ability to synthesize a library of different heterocycle-containing phenanthridinones could prove valuable in a world of evolving diseases, since heterocycles are sometimes more potent than non-heterocycle drugs. ${ }^{12}$ 


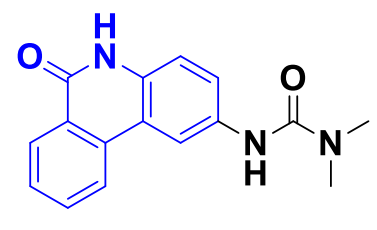

PJ34<smiles>O=c1c2cc(O)c(O)cc2c2cccc3ccn1c32</smiles>

Prastosine<smiles>Cc1ccc2c(=O)[nH]c3ccccc3c2c1</smiles>

Phenaglydon<smiles>O=c1[nH]c2ccccc2c2cc3c(cc12)OCO3</smiles>

Crinum asiaticum

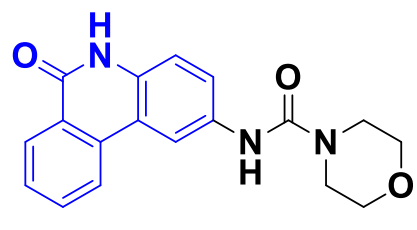

PJ38

Figure 1. Phenanthridinones in medicine and natural products.

Phenanthridinones are typically synthesized from traditional cross-coupling reactions such as the Suzuki-Miyaura coupling or Buchwald-Hartwig amination reaction. ${ }^{59}$ However, because of the inherent drawbacks associated with the required prefunctionalized starting materials, more recent efforts have led to oxidative variants with transition metals employing dehydrogenative coupling, yet these examples are limited to the use of simple arenes as coupling partners. ${ }^{60}$ However, Baidya and coworkers recently reported a copper-mediated dehydrogenative annulation of benzamides with 3,5-difluorpyridine (Scheme 17). ${ }^{61}$ 


\section{Sutton (Merck)}

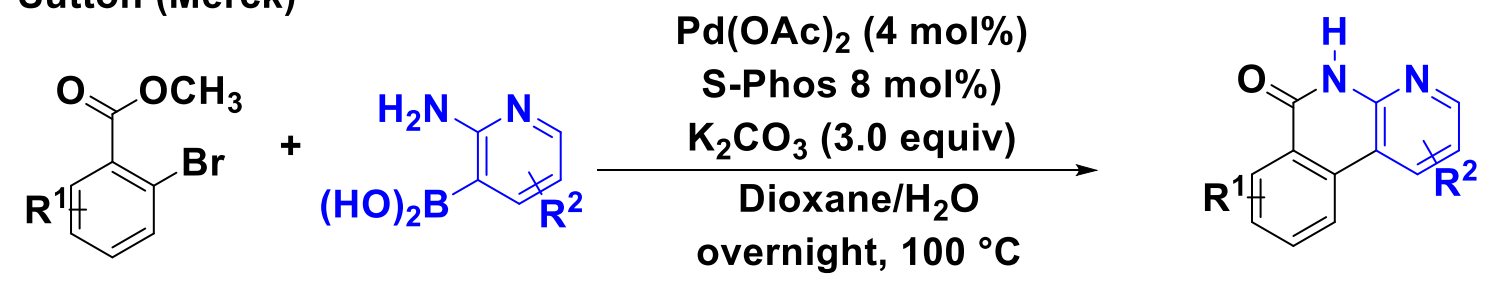

\section{Baidya}

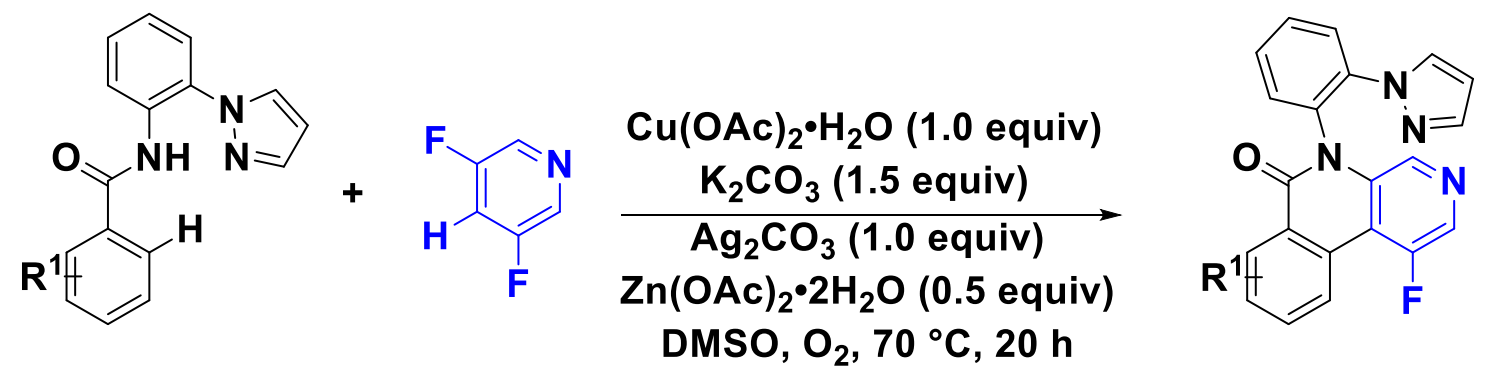

Scheme 17. Examples of heterocycle containing phenanthridinone syntheses.

Oxidative decarboxylative coupling reactions have been applied to the synthesis of phenanthridinones (Scheme 18). In 2014, Wang and co-workers reported the first synthesis of phenanthridinones utilizing a Pd-catalyzed ODC strategy with aryl acylperoxides and $N$-methoxybenzamides. ${ }^{62}$ However, despite the fair reaction yields, the method was limited to aryl acylperoxides, which are commercially limited. Similarly, Miura and co-workers reported an analogous copper-mediated ODC reaction for the synthesis of phenanthridinones from ortho-nitrobenzoates. ${ }^{65}$ While the work from Miura had advantages over Wang's (i.e. commercially available and shelf stable benzoates) both of their catalyst systems were limited to only 2-nitrobenzoates and aryl acylperoxides, heteroaryl carboxylates were ineffective coupling partners. 


\section{Wang}

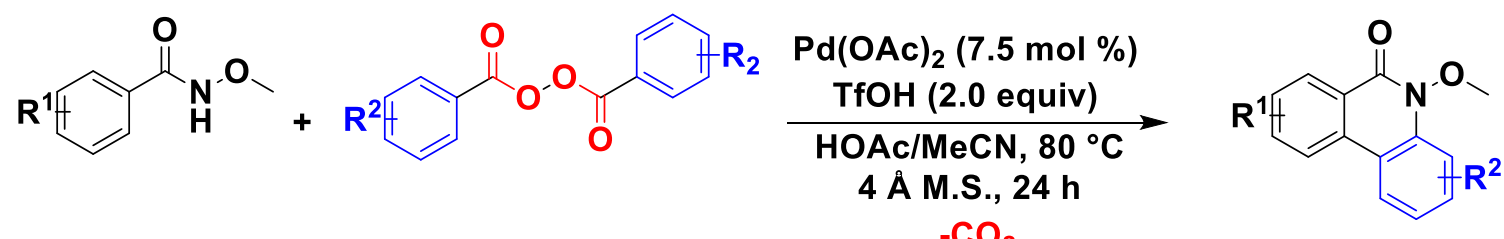

Miura

$-\mathrm{CO}_{2}$
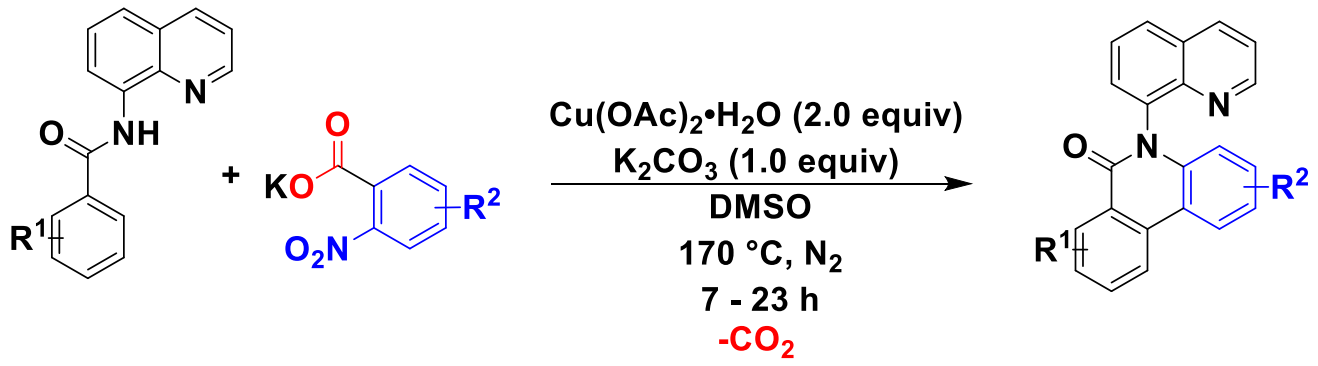

Scheme 18. Oxidative decarboxylative phenanthridinone syntheses.

At this time, there were no examples of a catalytic oxidative decarboxylative arylation and cyclization for the synthesis of heterocycle-containing phenanthridinones. From our studies in Chapter 2, we looked to extend our new methodology to couple heteroaromatic carboxylates with unactivated $\mathrm{C}-\mathrm{H}$ bonds (Scheme 19).

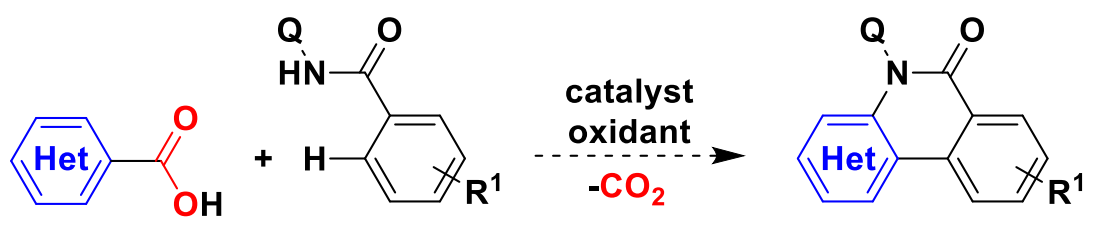

Scheme 19. Targeted goal of achieving a sequential oxidative decarboxylative heteroarylation and cyclization with unactivated $\mathrm{C}-\mathrm{H}$ bonds.

\subsection{Results}

\subsubsection{Optimization of the Sequential Oxidative Decarboxylative (Hetero)arylation and Cyclization Reaction}

We designed our reaction using 2-methyl- $N$-(quinolin-8-yl)benzamide and 2fluoronicotinic carboxylate in the presence of nickel and silver. We begin by using $\mathrm{Ni}(\mathrm{OAc})_{2} \bullet 4 \mathrm{H}_{2} \mathrm{O}$ as our pre-catalyst in the presence of $\mathrm{Ag}_{2} \mathrm{CO}_{3}$ as our oxidant which generated the desired product $\mathbf{5 a}$ in $25 \%$ yield (Table 3.1 entry 1). All other nickel salts tested provided low yields of the desired product in $(<10 \%) . \mathrm{AgNO}_{3}$ was the best oxidant 
for this transformation when the loading was increased to 4.0 equivalents, while other oxidants explored (AgOPiv and AgOAc) gave lower yields (Table 3.1 entry 3 and 4). Finally, when we increased the loading of 5a from 1.5 equivalents to 2.0 equivalents, the yield of the desired product increased to $81 \%$ (Table 3.1 entry 6). Therefore, the final optimized conditions employ $20 \mathrm{~mol} \%$ of $\mathrm{Ni}(\mathrm{OAc})_{2} \bullet 4 \mathrm{H}_{2} \mathrm{O}, \mathrm{AgNO}_{3}$ (4.0 equiv), and $\mathrm{Na}_{2} \mathrm{CO}_{3}$ (4.0 equiv) in DMA at $130{ }^{\circ} \mathrm{C}$ for $24 \mathrm{~h}$.

Table 3.1. Optimization of the nickel-catalyzed sequential oxidative decarboxylative (hetero)arylation ${ }^{a}$

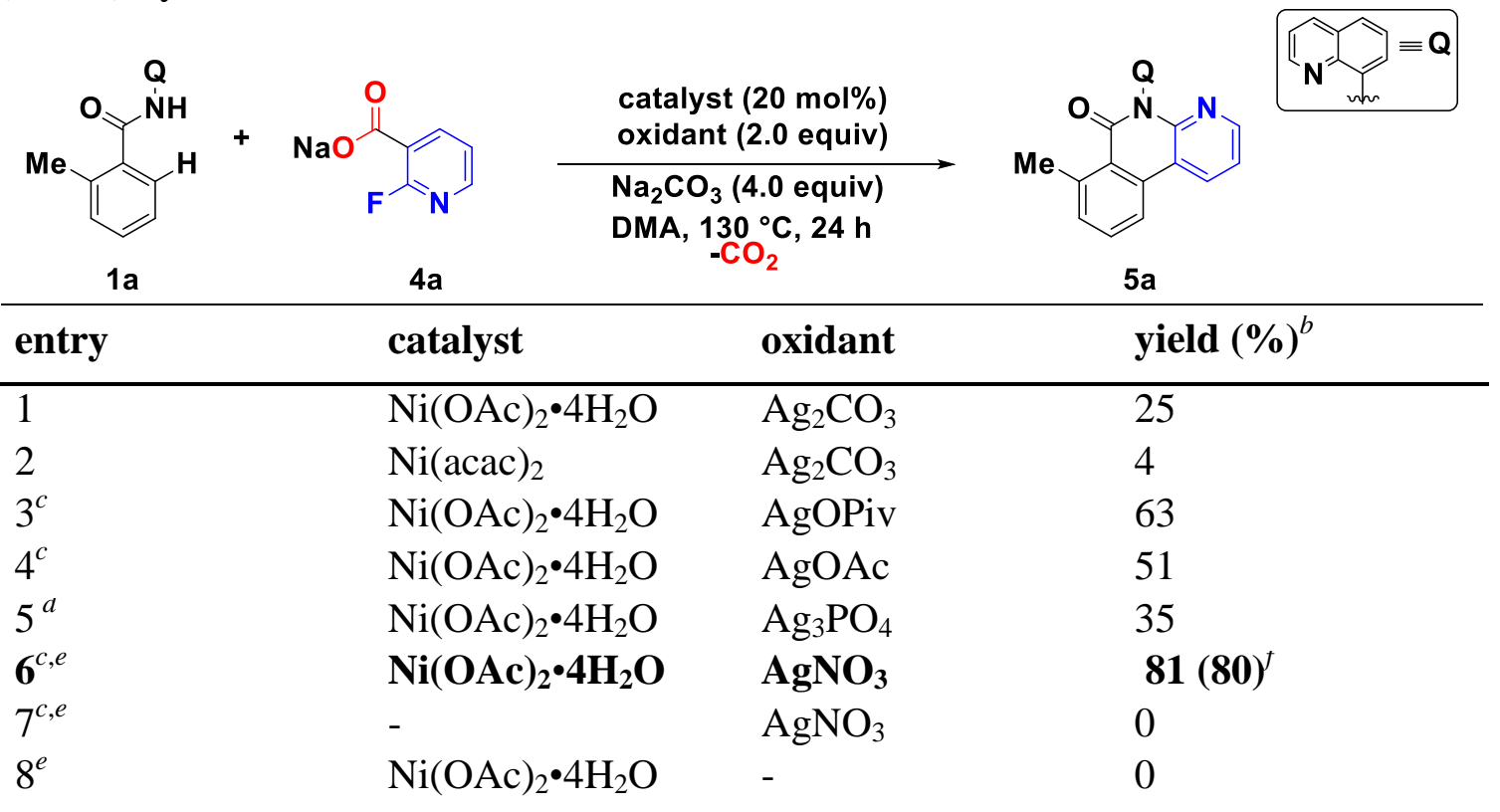

${ }^{a}$ Reaction conditions: 1a $(0.2 \mathrm{mmol}), 4 \mathbf{a}(0.3 \mathrm{mmol})$ in DMA $(2 \mathrm{~mL}) .{ }^{b 1} \mathrm{H}$ NMR yield with 1,3,5-trimethoxybenzene as an internal standard. ${ }^{c} \mathrm{Ag}$ (4.0 equiv). ${ }^{d} 1.3$ equiv $\mathrm{Ag}_{3} \mathrm{PO}_{4}$ (4.0 equiv of $\mathrm{Ag})^{e} \mathbf{4 a}(0.4 \mathrm{mmol}) .{ }^{f}$ Isolated yield.

\subsubsection{Scope of the Sequential Oxidative Decarboxylative (Hetero)arylation and Cyclization Reaction}

With the optimized conditions in hand, we turned our attention to the heteroaromatic carboxylate scope (Table 3.2). The reaction is compatible with both electron-withdrawing (5d, 5e, and 5g), and electron-donating (5b, 5c, and 5f) substituents on the nicotinic carboxylate. We found that substituents in the $\mathrm{C}-6$ position of the nicotinate resulted in lower yields $(\mathbf{4 c}-\mathbf{4 e})$ than their unsubstituted counterpart (4a). Analogous to the ODC reaction described in Chapter 2, this new catalyst system also tolerates bromo- and 
chloro-substitution (5e and $\mathbf{5 g}$ ) allowing for further functionalization in subsequent crosscoupling reactions. This new catalyst method could also be conducted on a $1 \mathrm{mmol}$ scale without a significant reduction in yield (75\% of 5a). To our delight, we found that other heteroaromatic carboxylates also couple under this new reaction system. Quinoline and thiophenes are found in pharmacologically active compounds ${ }^{63}$ and material sciences ${ }^{64}$ and the corresponding carboxylates $(\mathbf{5 h}-\mathbf{5 j})$ also proved to be competent coupling partners under these catalytic conditions.

Table 3.2. Ni-catalyzed oxidative decarboxylative arylation and cyclization heteroaromatic carboxylate scope ${ }^{a}$

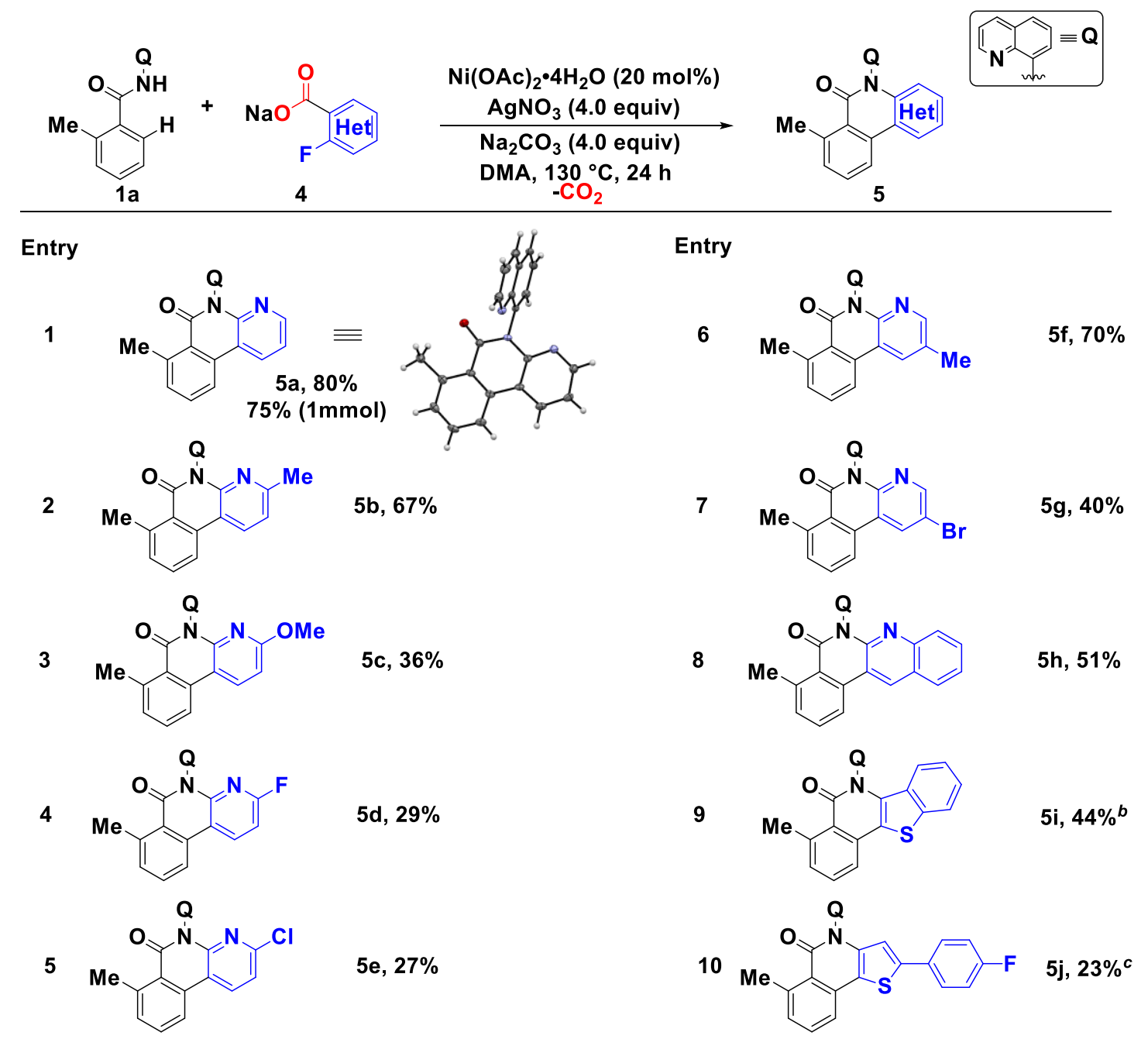

\footnotetext{
${ }^{a}$ Isolated yields. Reaction conditions: 1a $(0.2 \mathrm{mmol}), 4(0.4 \mathrm{mmol})$ in DMA $(2 \mathrm{~mL}) .{ }^{b} 130$ ${ }^{\circ} \mathrm{C}$ for $8 \mathrm{~h}$ then $170{ }^{\circ} \mathrm{C}$ for $16 \mathrm{~h}^{c} 170{ }^{\circ} \mathrm{C}$.
} 
This new catalyst system also worked with substituted benzoates as seen in our previous ODC reaction (Table 3.3). To our surprise, the sequential oxidative decarboxylative arylation proceeded smoothly at $170{ }^{\circ} \mathrm{C}$. A variety of different electrondonating (5l and 5o) and electron-withdrawing groups (5k, 5m, and $\mathbf{5 n})$ were tolerated on the benzoates. However, we did notice di-ortho-substituted 2-fluoro-6-methylbenzaote (4p) was an ineffective coupling partner under these reaction conditions. This new method illustrates the ability to couple and cyclize not only heteroaromatic carboxylates but also ortho-fluorobenzoates.

Table 3.3. Ni-catalyzed oxidative decarboxylative arylation and cyclization benzoate scope $^{a}$

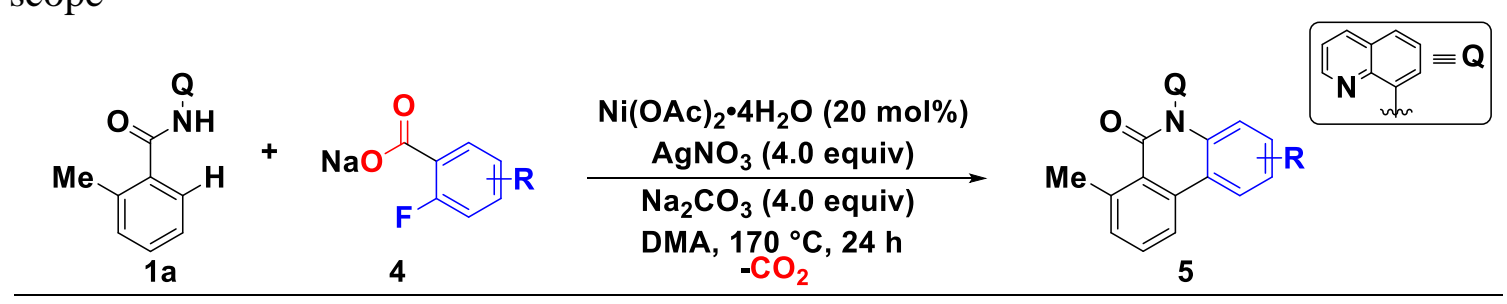

Entry

1<smiles>Cc1cccc2c1c(=O)n(C)c1ccccc21</smiles>

2<smiles>COc1cccc2c1c(=O)[nH]c1ccc(F)cc12</smiles>

3<smiles>Cc1ccc2c(c1)c1cccc(C)c1c(=O)n2C</smiles><smiles>Cc1cccc2c1c(=O)n(C)c1cc(Br)ccc21</smiles>

$5 j, 68 \%$

$5 k, 62 \%$

5I, $56 \%$

$5 \mathrm{~m}, 36 \%$

\section{Entry}

5<smiles>Cc1cccc2c1c(=O)n(C)c1cc(F)ccc21</smiles>

$5 n, 68 \%$

6

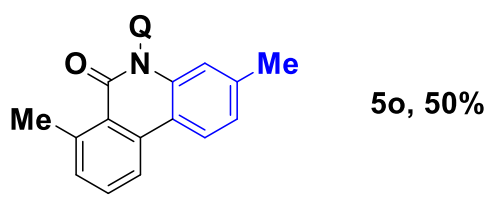

7<smiles>Cc1cccc2c1c(=O)n(C)c1cccc(C)c21</smiles>

$5 p, 0 \%$

\footnotetext{
${ }^{a}$ Isolated yields. Reaction conditions: 1a $(0.2 \mathrm{mmol}), 4(0.4 \mathrm{mmol})$ in DMA $(2 \mathrm{~mL})$.
}

Finally, we explored the scope of the substituted benzamides with the 2-fluoronicotinic carboxylate (Table 3.4). Benzamide substrates with electron-donating groups para to the 
benzamide moiety (5q and $\mathbf{5 r}$ ) gave higher yields than the electron-withdrawing group (5s). In addition, substrates bearing electron-donating groups in the meta-position (5t and 5u) gave higher yields (54\% and 70\%) than those with electron-withdrawing groups ( $\mathbf{5 v}$, $31 \%)$. We did notice that in the absence of an ortho-substituent on the benzamide, product formation was reduced $(\mathbf{5 w})$. This new catalyst method also tolerates heterocyclic benzamides $(\mathbf{5 x})$ opening the potential to build a vast library of substituted phenanthridinones.

Table 3.4. Ni-catalyzed oxidative decarboxylative arylation and cyclization benzamide scope $^{a}$

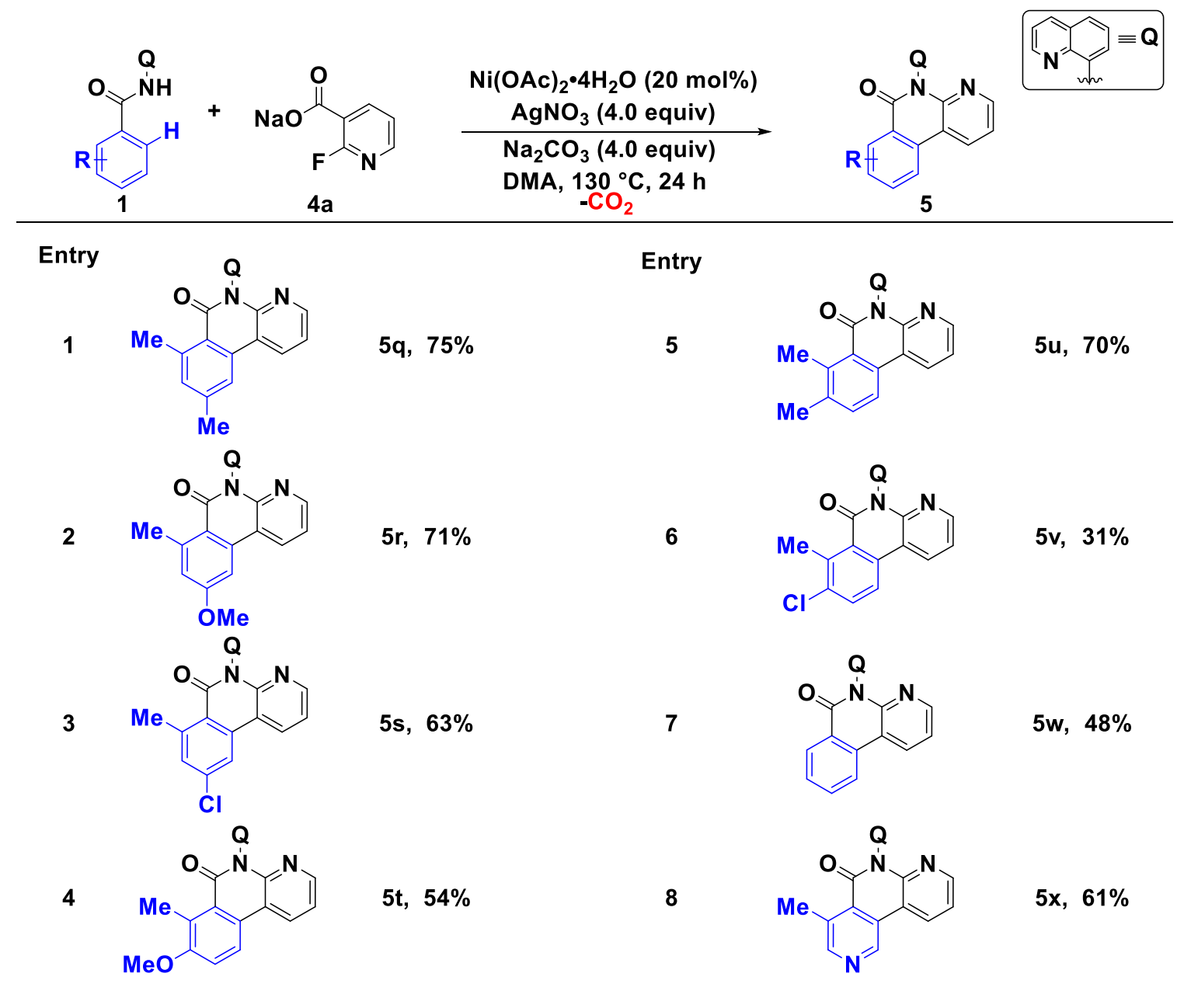

\footnotetext{
${ }^{a}$ Isolated yields. Reaction conditions: $1(0.2 \mathrm{mmol}), \mathbf{4 a}(0.4 \mathrm{mmol})$ in DMA $(2 \mathrm{~mL})$.
} 


\subsubsection{Preliminary Mechanistic Studies of the Sequential Oxidative Decarboxylative (Hetero)Arylation and Cyclization Reaction}

First, we conducted a series of radical trapping experiments under our reaction conditions to explore the possible formation of free radical intermediates. The reaction of 1a and $4 \mathbf{a}$ in the presence of 1.0 equiv of either TEMPO or DHA only slightly decreased the yield of the desired product 5a (Table 3.5, entry 2 and 3). These data suggest there could be a well-defined silver-aryl intermediate in this reaction, analogous to the ODC reaction reported above (Chapter 2).

Table 3.5. Radical scavengers with the nickel-catalyzed sequential oxidative decarboxylative (hetero)arylation and cyclization reaction ${ }^{a}$

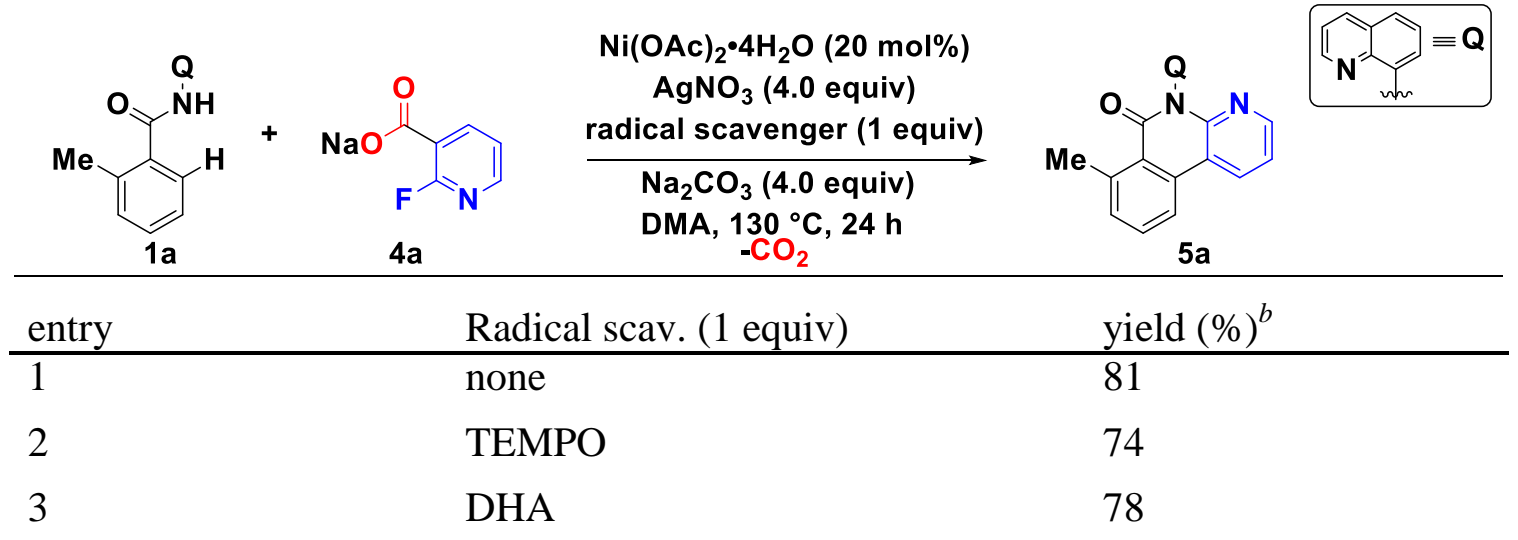

${ }^{a}$ Reaction conditions: Benzamide 1a $(0.2 \mathrm{mmol})$, heteroaromatic carboxylate 4a $(0.4$ mmol), $\mathrm{Ni}(\mathrm{OAc})_{2} \bullet 4 \mathrm{H}_{2} \mathrm{O}(20 \mathrm{~mol} \%), \mathrm{AgNO}_{3}$ (4.0 equiv), $\mathrm{Na}_{2} \mathrm{CO}_{3}$ (4.0 equiv), radical scavenger (1.0 equiv) in DMA ( $2 \mathrm{~mL}$ ) for $24 \mathrm{~h}$ at $130{ }^{\circ} \mathrm{C}$ under a $\mathrm{N}_{2}$ atmosphere. ${ }^{b 1} \mathrm{H}$ NMR yield with 1,3,5-trimethoxybenzene as an internal standard.

In order to gain insight into the $\mathrm{C}-\mathrm{H}$ activation step, we carried out a deuterium/hydrogen exchange experiment. We measured the KIE from an intermolecular competition experiment with an equimolar mixture of 1a and 1a- $\boldsymbol{d}_{7}$ under our standard reaction conditions for 30 minutes. The KIE determined from the resulting mixture of $\mathbf{5 a}$ and $\mathbf{5 a}-\boldsymbol{d}_{7}$ products is 4.5 (Scheme 20). Next, we carried out the deuterium/hydrogen exchange experiment of $\mathbf{1 a}-\boldsymbol{d}_{7}$ under the standard conditions for $30 \mathrm{~min}$., the D content only dropped by $>1 \%$. These data indicate a primary isotope effect and that the $\mathrm{C}-\mathrm{H}$ activation is irreversible. 
Scheme 20. Kinetic isotope effect of the nickel-catalyzed sequential oxidative decarboxylative.

(a) Intermolecular KIE Experiment ${ }^{a, c}$<smiles>Cc1ccccc1C(=O)NC(=O)c1ccccc1</smiles>

$1 \mathbf{a}$

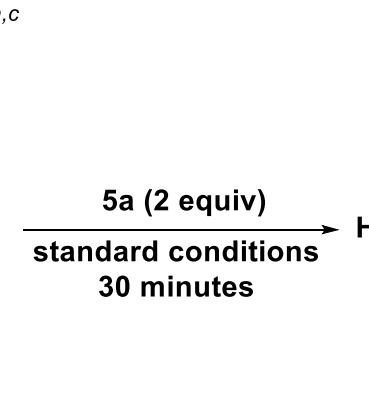

(b) Deuterium-Exchange Experiment ${ }^{b, c}$

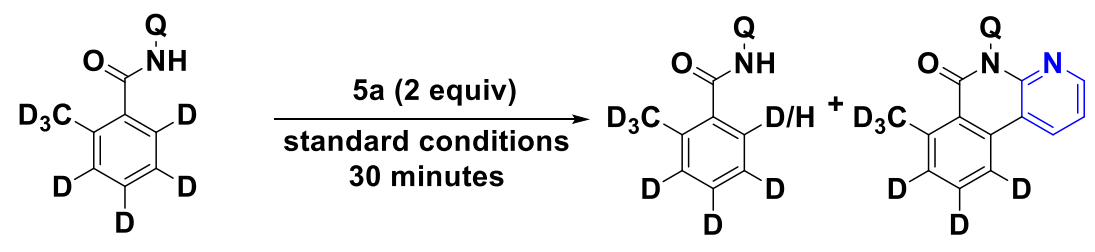

$1 \mathrm{a}-\mathrm{d}_{7}$

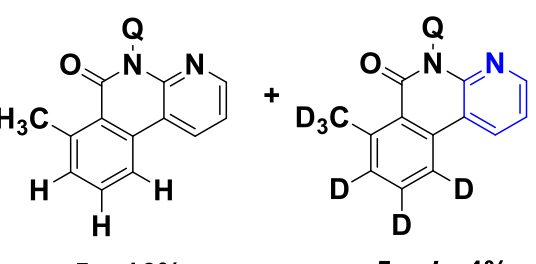

$5 a, 16 \%$

$5 a-d_{7}, 4 \%$

intermolecular KIE $=4.5$

Reaction conditions: Benzamide $\mathbf{1 a}(0.1 \mathrm{mmol}), \mathbf{1 a}-\boldsymbol{d}_{\mathbf{7}}(0.1 \mathrm{mmol})$, heteroaromatic carboxylate $4 \mathbf{a}(0.4 \mathrm{mmol}), \mathrm{Ni}(\mathrm{OAc})_{2} \bullet 4 \mathrm{H}_{2} \mathrm{O}(20 \mathrm{~mol} \%), \mathrm{AgNO}_{3}$ (4.0 equiv), $\mathrm{Na}_{2} \mathrm{CO}_{3}$ (4.0 equiv) in DMA ( $2 \mathrm{~mL}$ ) for $30 \mathrm{~min}$. at $130{ }^{\circ} \mathrm{C}$ under a $\mathrm{N}_{2}$ atmosphere. ${ }^{b}$ Benzamide $\mathbf{~ l a}_{-} \boldsymbol{d}_{7}(0.2 \mathrm{mmol})$, heteroaromatic carboxylate $4 \mathbf{a}(0.4 \mathrm{mmol}), \mathrm{Ni}(\mathrm{OAc})_{2} \bullet 4 \mathrm{H}_{2} \mathrm{O}(20 \mathrm{~mol}$ $\%$ ), $\mathrm{AgNO}_{3}$ (4.0 equiv), $\mathrm{Na}_{2} \mathrm{CO}_{3}$ (4.0 equiv) in DMA (2 mL) for $30 \mathrm{~min}$. at $130{ }^{\circ} \mathrm{C}$ under a $\mathrm{N}_{2}$ atmosphere. ${ }^{c 1} \mathrm{H}$ NMR yield with 1,3,5-trimethoxybenzene as an internal standard.

We found that $\mathrm{Na}_{2} \mathrm{CO}_{3}$ promotes a $\mathrm{S}_{\mathrm{N}}$ Ar-type cyclization following the oxidative decarboxylative coupling. From our ODC reaction reported above (Chapter 2), we prepared 2'-fluoro-3-methyl- $N$-(quinolin-8-yl)-[1,1'-biphenyl]-2-carboxamide (3r) and heated the compound at $170{ }^{\circ} \mathrm{C}$ in the presence of $\mathrm{Na}_{2} \mathrm{CO}_{3}$ to mimic our benzoate reaction conditions to see if the compound would undergo cyclization. To our delight, compound $3 \mathbf{r}$ underwent a $S_{N} A r$ cyclization to generate the desired product $(\mathbf{5 k})$ in quantitative yield (Scheme 21). This type of cyclization has been seen before in other reactions. ${ }^{65}$ 
Scheme 21. Base promoted cyclization by $S_{\mathrm{N}} \mathrm{Ar}^{a}$<smiles>Cc1cccc(-c2ccccc2F)c1C(=O)N=O</smiles>

$3 r$

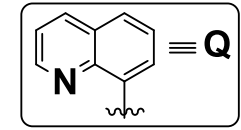

Q<smiles></smiles>

$5 \mathrm{k},>95 \%$ yield $^{b}$

${ }^{a}$ Reaction conditions: 2'-fluoro-3-methyl-N-(quinolin-8-yl)-[1,1'-biphenyl]-2carboxamide $3 \mathbf{r}(0.2 \mathrm{mmol})$ and $\mathrm{Na}_{2} \mathrm{CO}_{3}\left(4.0\right.$ equiv) in DMA $(2 \mathrm{~mL})$ for $24 \mathrm{~h}$ at $170{ }^{\circ} \mathrm{C}$ under a $\mathrm{N}_{2}$ atmosphere. ${ }^{b 1} \mathrm{H}$ NMR yield with 1,3,5-trimethoxybenzene as an internal standard.

\subsubsection{Proposed Reaction Mechanism of the Sequential Oxidative Decarboxylative (Hetero)Arylation and Cyclization Reaction}

Based on the results above, we proposed a pathway for this new catalytic transformation that follows closely with that proposed for the Ni-catalyzed ODC reaction (Scheme 22). The catalyst undergoes ligand exchange with the amide and then $\mathrm{C}-\mathrm{H}$ activation to form the nickel(II) metallacycle B. Transmetallation of the aryl fragment to the nickel(II) metallacycle with concomitant oxidation of nickel(II) to nickel(III) to form intermediate C. After reductive elimination of the high-valent nickel(III) intermediate to from the new $\mathrm{C}-\mathrm{C}$ bond resulting in complex $\mathbf{D}$. The desired product is then formed by $\mathrm{S}_{\mathrm{N}} \mathrm{Ar}$ cyclization. 
Scheme 22. Proposed reaction mechanism of the sequential oxidative decarboxylative (hetero)arylation and cyclization reaction.

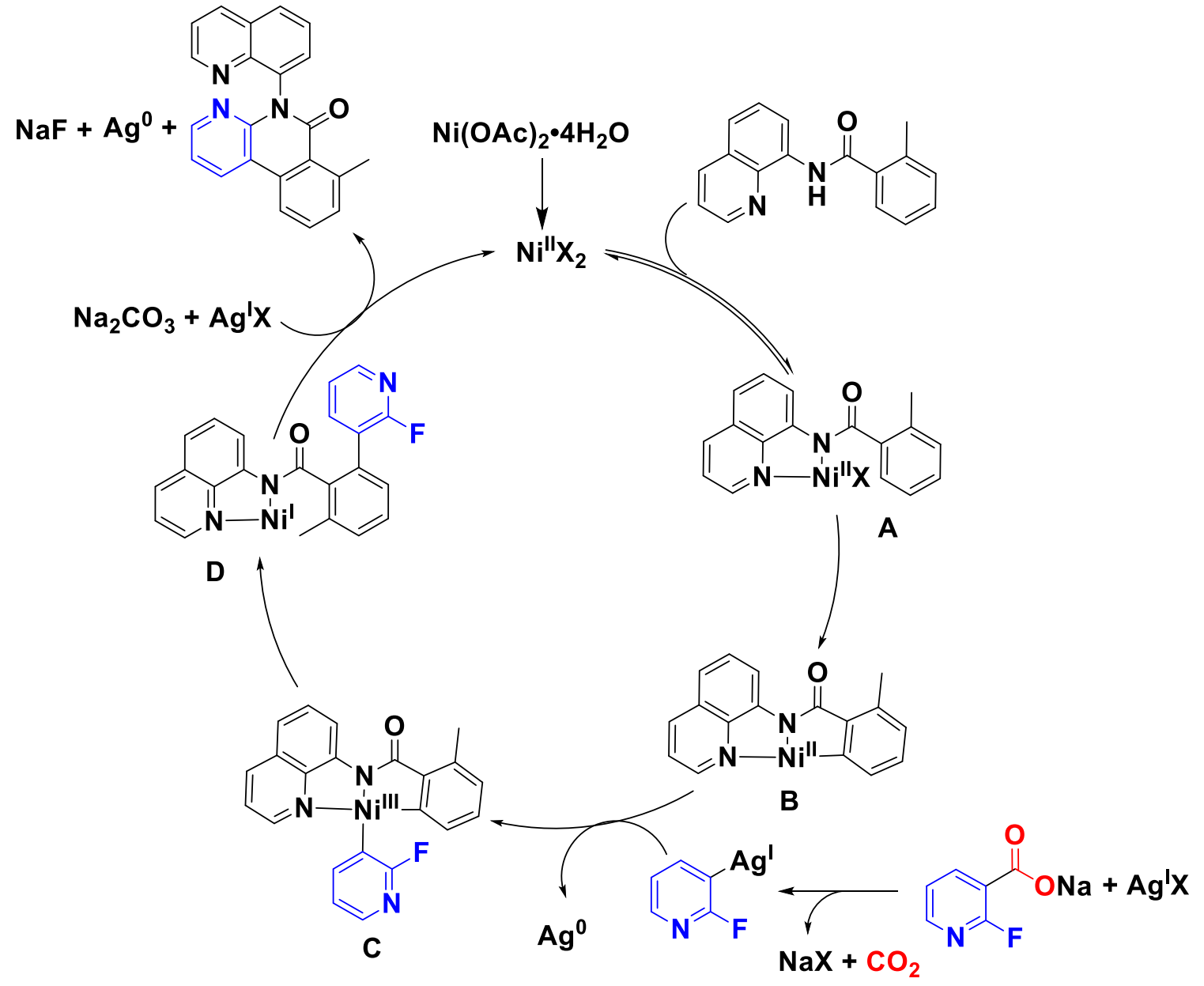

\subsection{Conclusion}

In conclusion, we have developed a new method for the synthesis of heterocyclecontaining phenanthridinones via an oxidative decarboxylative annulation reaction. This new method was not only effective for the coupling of heteroaromatic carboxylates but also ortho-fluoro benzoates. This new method could allow for the synthesis of a library of heterocycle-containing phenanthridinones with biological properties. 


\subsection{Experimental}

General Considerations. All manipulations were performed using standard Schlenk or glovebox techniques under a nitrogen atmosphere. All solvents (including dry DMA) were purchased from Alfa-Aesar, Fisher, or Cambridge Isotope Laboratories (deuterated solvents) and used as received. All other reagents were purchased from Maybridge, Oakwood, Acros, Alfa-Aesar, Astatech, Strem and CDN Isotopes and used without further purification. All NMR spectra were recorded at ambient temperature on an Agilent $400 \mathrm{MHz}$ or JEOL $400 \mathrm{MHz}\left({ }^{1} \mathrm{H}, 400 \mathrm{MHz} ;{ }^{13} \mathrm{C}\left\{{ }^{1} \mathrm{H}\right\}, 100 \mathrm{MHz} ;{ }^{19} \mathrm{~F}, 376 \mathrm{MHz}\right)$ spectrometer. Chemical shifts are referenced to the residual solvent signals $\left(\mathrm{CDCl}_{3}: 7.26\right.$ ppm $\left({ }^{1} \mathrm{H}\right)$ and $77.2 \mathrm{ppm}\left({ }^{13} \mathrm{C}\right)$, DMSO- $d_{6}: 2.50 \mathrm{ppm}\left({ }^{1} \mathrm{H}\right)$ and $\left.39.5 \mathrm{ppm}\left({ }^{13} \mathrm{C}\right)\right) .{ }^{45} \mathrm{High}$ resolution mass spectra were obtained on a Thermofisher Scientific Q Exactive Mass Spectrometer. IR spectra were recorded on a PerkinElmer (Spectrum 100) FT-IR spectrometer. Column chromatography was performed using Silicycle Silica Flash P60 silica gel.

\section{Optimization of the Oxidative Decarboxylative Annulation Reaction}

\section{Representative Procedure for the Optimization of the Oxidative Decarboxylative} Annulation Reaction. An oven-dried $50 \mathrm{~mL}$ Schlenk tube with a stirring bar was charged with 2-fluoronicotinic acid $\mathbf{4 a}(42.3 \mathrm{mg}, 0.300 \mathrm{mmol})$, sodium carbonate (31.7 $\mathrm{mg}, 0.300 \mathrm{mmol})$, and dry DMA (1 mL). The reaction vessel was placed in a pre-heated oil bath and stirred for $0.5 \mathrm{~h}$ at $110{ }^{\circ} \mathrm{C}$. The solvent was removed under reduced pressure until dry. 2-Methyl- $N$-(quinolin-8-yl) benzamide $1 \mathrm{a}(52.4 \mathrm{mg}, 0.200 \mathrm{mmol})$, $\mathrm{Ni}(\mathrm{OAc})_{2} \bullet 4 \mathrm{H}_{2} \mathrm{O}(9.9 \mathrm{mg}, 0.040 \mathrm{mmol}), \mathrm{Ag}_{2} \mathrm{CO}_{3}(110 \mathrm{mg}, 0.400 \mathrm{mmol}), \mathrm{Na}_{2} \mathrm{CO}_{3}(84 \mathrm{mg}$, $0.80 \mathrm{mmol}$ ) were added and the tube was evacuated and backfilled with nitrogen three times after which dry DMA (2 $\mathrm{mL})$ was added via syringe. The reaction mixture was stirred at $130{ }^{\circ} \mathrm{C}$ for $24 \mathrm{~h}$. Upon completion, the reaction tube was cooled to room temperature. The solution was diluted with dichloromethane $(30 \mathrm{~mL})$ and filtered through a pad of celite. The celite was then washed with dichloromethane $(2 \times 30 \mathrm{~mL})$. The solvent was concentrated by rotary evaporation (to $\sim 2 \mathrm{~mL}$ ) and the remaining solvent was 
removed under vacuum. A 1,3,5-trimethoxybenzene (5.00 mg) NMR standard was added to the residue and the crude mixture was dissolved in $\mathrm{CDCl}_{3}$ for ${ }^{1} \mathrm{H} \mathrm{NMR}$ analysis.

\section{Optimization of the Precatalyst ${ }^{a}$}<smiles>Cc1cccc(F)c1C(=O)Nc1cccc2cccnc12</smiles>

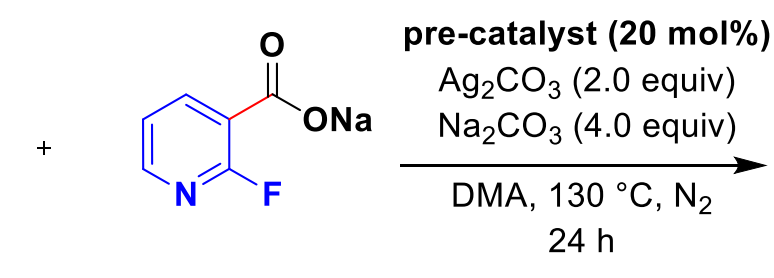

4a

1a<smiles>Cc1cccc2c1c(=O)n(-c1cccc3cccnc13)c1ncccc21</smiles>

$5 a$

\begin{tabular}{lll}
\hline entry & pre-catalyst & yield 5a $(\%)^{b}$ \\
\hline 1 & $\mathrm{NiBr}_{2} \bullet \mathrm{H}_{2} \mathrm{O}$ & 3 \\
2 & $\mathrm{Ni}\left(\mathrm{HCO}_{2}\right)_{2} \bullet 2 \mathrm{H}_{2} \mathrm{O}$ & 6 \\
3 & $\mathrm{Ni}(\mathrm{acac})_{2}$ & 4 \\
4 & $\mathrm{NiI}_{2}$ & 9 \\
5 & $\mathrm{NiCO}_{3} \bullet 2 \mathrm{Ni}(\mathrm{OH})_{2} \bullet \mathrm{H}_{2} \mathrm{O}$ & 1 \\
6 & $\left.\mathrm{Ni}_{(\mathrm{OTf}}\right)_{2}$ & 3 \\
7 & $\mathrm{NiF}_{2}$ & 1 \\
8 & $\mathrm{NiCl}_{2}$ & 5 \\
9 & $\mathrm{Ni}(\mathrm{OAc})_{2} \bullet 4 \mathrm{H}_{2} \mathrm{O}$ & 63 \\
$10^{c}$ & $\mathrm{Ni}(\mathrm{OAc})_{2} \bullet 4 \mathrm{H}_{2} \mathrm{O}$ & 6 \\
$11^{d}$ & $\mathrm{Ni}(\mathrm{OAc})_{2} \bullet 4 \mathrm{H}_{2} \mathrm{O}$ & 57 \\
12 & none & 0 \\
\hline
\end{tabular}

${ }^{a}$ Reaction conditions: 1a $(0.200 \mathrm{mmol}), \mathbf{4 a}(0.300 \mathrm{mmol})$ in DMA $(2 \mathrm{~mL}) .{ }^{b 1} \mathrm{H} \mathrm{NMR}$ yield determined from integration using 1,3,5-trimethoxybenzene as an internal standard. ${ }^{c} 10 \mathrm{~mol} \%{ }^{d} 30 \mathrm{~mol} \%$ 


\section{Optimization of the Oxidant ${ }^{a}$}<smiles>Cc1ccccc1C(=O)Nc1cccc2cccnc12</smiles>

$1 \mathrm{a}$

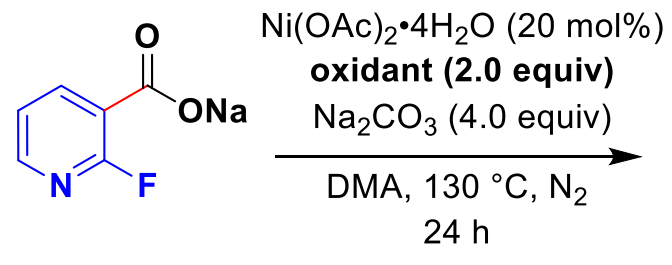

4a

\begin{tabular}{|c|c|c|}
\hline entry & oxidant & yield $\mathbf{5 a}(\%)^{b}$ \\
\hline 1 & $\mathrm{Ag}_{2} \mathrm{CO}_{3}$ & 63 \\
\hline $2^{c}$ & $\mathrm{AgNO}_{3}$ & 72 \\
\hline $3^{c}$ & $\mathrm{AgOAc}$ & 51 \\
\hline $4^{c}$ & AgOPiv & 63 \\
\hline $5^{f}$ & $\mathrm{Ag}_{3} \mathrm{PO}_{4}$ & 35 \\
\hline 6 & $\mathrm{Ag}_{2} \mathrm{O}$ & 18 \\
\hline 7 & oxone & 0 \\
\hline 8 & $\mathrm{O}_{2}(1 \mathrm{~atm})$ & 0 \\
\hline $9^{d}$ & $\mathrm{AgNO}_{3}$ & 56 \\
\hline $10^{e}$ & $\mathrm{AgNO}_{3}$ & 62 \\
\hline 11 & none & 0 \\
\hline
\end{tabular}<smiles>Cc1cccc2c1c(=O)n(-c1cccc3cccnc13)c1ncccc21</smiles>

$5 a$

${ }^{a}$ Reaction conditions: 1a $(0.200 \mathrm{mmol}), \mathbf{4 a}(0.300 \mathrm{mmol})$ in DMA $(2 \mathrm{~mL}) .{ }^{b 1} \mathrm{H} \mathrm{NMR}$ yield determined from integration using 1,3,5-trimethoxybenzene as an internal standard. ${ }^{\mathrm{c}} \mathrm{Ag}$ (4.0 equiv) ${ }^{d} 3.0$ equiv $^{e} 5.0$ equiv ${ }^{f \mathrm{~d}} 1.3$ equiv $\mathrm{Ag}_{3} \mathrm{PO}_{4}$ (4.0 equiv of $\mathrm{Ag}$ ) 


\section{Optimization of the Solvent ${ }^{a}$}<smiles>Cc1ccccc1C(=O)Nc1cccc2cccnc12</smiles>

$1 a$

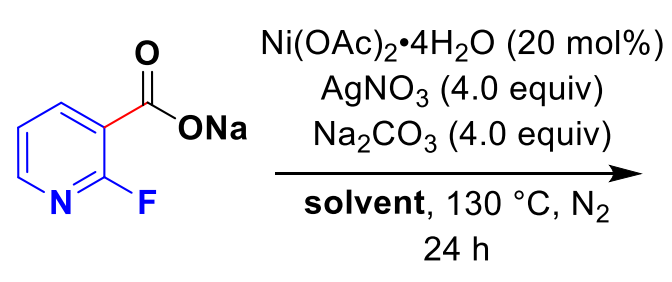

$4 a$

\begin{tabular}{|c|c|c|}
\hline entry & solvent & yield $\mathbf{5 a}(\%)^{b}$ \\
\hline 1 & DMA & 72 \\
\hline 2 & $\mathrm{DMF}$ & 52 \\
\hline 3 & NMP & 63 \\
\hline 4 & DMSO & 10 \\
\hline 5 & $\mathrm{PhCH}_{3}$ & 0 \\
\hline 6 & $\mathrm{MeCN}$ & 0 \\
\hline $7^{c}$ & DMA & 38 \\
\hline $8^{d}$ & DMA & 73 \\
\hline
\end{tabular}<smiles>Cc1cccc2c1c(=O)n(-c1cccc3cccnc13)c1ncccc21</smiles>

$5 a$

${ }^{a}$ Reaction conditions: 1a $(0.200 \mathrm{mmol}), \mathbf{4 a}(0.300 \mathrm{mmol})$ in solvent $(2 \mathrm{~mL}) .{ }^{b 1} \mathrm{H}$ NMR yield determined from integration using 1,3,5-trimethoxybenzene as an internal standard. ${ }^{c} 1 \mathrm{~mL}^{d} 3 \mathrm{~mL}$ 


\section{Optimization of the Base ${ }^{a}$}<smiles>Cc1ccccc1C(=O)Nc1cccc2cccnc12</smiles>

$1 a$

$4 a$

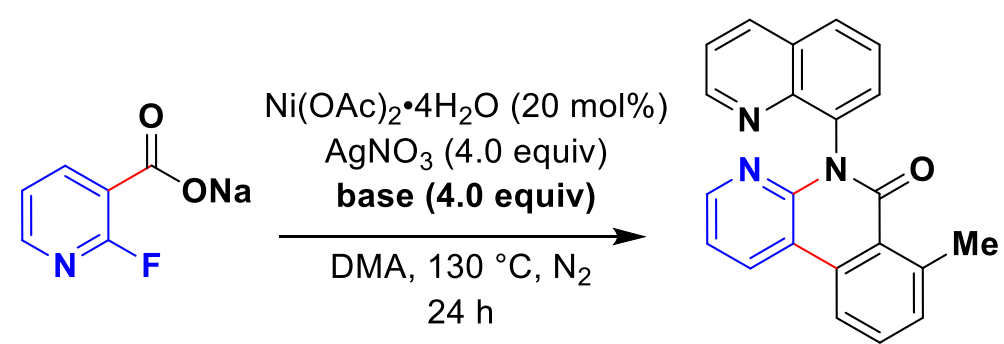

$5 a$

\begin{tabular}{lll}
\hline entry & base & yield 5a $(\%)^{b}$ \\
\hline 1 & $\mathrm{Li}_{2} \mathrm{CO}_{3}$ & 53 \\
2 & $\mathrm{Na}_{2} \mathrm{CO}_{3}$ & 72 \\
3 & $\mathrm{~K}_{2} \mathrm{CO}_{3}$ & 18 \\
4 & $\mathrm{NaOAc}$ & 40 \\
5 & pyridine & 47 \\
6 & none & 29 \\
\hline
\end{tabular}

${ }^{a}$ Reaction conditions: 1a $(0.200 \mathrm{mmol}), \mathbf{4 a}(0.300 \mathrm{mmol})$ in DMA $(2 \mathrm{~mL}) .{ }^{b 1} \mathrm{H}$ NMR yield determined from integration using 1,3,5-trimethoxybenzene as an internal standard.

\section{Optimization of the Temperature ${ }^{a}$}<smiles>Cc1ccccc1C(=O)Nc1cccc2cccnc12</smiles>

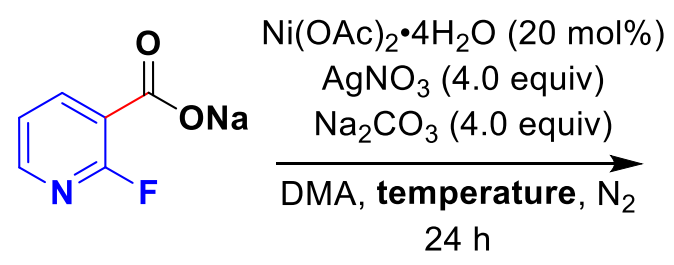

$4 a$

\begin{tabular}{lll}
\hline entry & Temperature $\left({ }^{\circ} \mathrm{C}\right)$ & yield $\mathbf{5 a}(\%)^{b}$ \\
\hline 1 & 120 & 56 \\
2 & 130 & 72 \\
3 & 140 & 73 \\
4 & 150 & 73 \\
\hline
\end{tabular}<smiles>Cc1cccc2c1c(=O)n(-c1cccc3cccnc13)c1ncccc21</smiles>

$5 a$

${ }^{a}$ Reaction conditions: 1a $(0.200 \mathrm{mmol}), \mathbf{4 a}(0.300 \mathrm{mmol})$ in DMA $(2 \mathrm{~mL}) .{ }^{b 1} \mathrm{H} \mathrm{NMR}$ yield determined from integration using 1,3,5-trimethoxybenzene as an internal standard. 
Equivalents of $4 \mathbf{a}^{a}$<smiles>Cc1ccccc1C(=O)Nc1cccc2cccnc12</smiles>

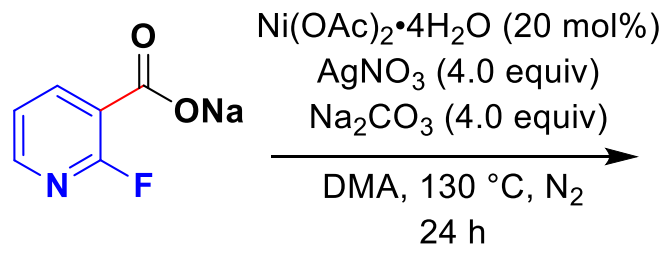

$24 \mathrm{~h}$<smiles>Cc1cccc2c1c(=O)n(-c1cccc3cccnc13)c1ncccc21</smiles>

$5 a$

$1 \mathrm{a}$

\begin{tabular}{lll}
\multicolumn{4}{c}{$\mathbf{a}$} & \\
\hline entry & $\mathbf{4 a}$ & yield $\mathbf{5 a}(\%)^{b}$ \\
\hline 1 & 1.5 equiv & 73 \\
2 & 2.0 equiv & 81 \\
3 & 3.0 equiv & 81 \\
\hline
\end{tabular}

${ }^{a}$ Reaction conditions: 1a $(0.200 \mathrm{mmol}), \mathbf{4 a}$ in DMA $(200 \mathrm{~mL}) .{ }^{b 1} \mathrm{H} \mathrm{NMR}$ yield determined from integration using 1,3,5-trimethoxybenzene as an internal standard.

\section{General Procedure for the Oxidative Decarboxylative Annulation Reaction}

Procedure A: An oven-dried $50 \mathrm{~mL}$ Schlenk tube with a stirring bar was charged with carboxylic acid 4 (0.400 mmol), $\mathrm{Na}_{2} \mathrm{CO}_{3}(42.3 \mathrm{mg}, 0.400 \mathrm{mmol})$, and dry DMA (1 mL). The reaction vessel was placed in a pre-heated oil bath and stirred for $0.5 \mathrm{~h}$ at $110{ }^{\circ} \mathrm{C}$. The solvent was then removed under reduced pressure until dry and the carboxylate salt was used without further purification. The corresponding benzamide $1(0.200 \mathrm{mmol})$, $\mathrm{Ni}(\mathrm{OAc})_{2} \bullet 4 \mathrm{H}_{2} \mathrm{O}(9.9 \mathrm{mg}, 0.040 \mathrm{mmol}), \mathrm{AgNO}_{3}(136 \mathrm{mg}, 0.800 \mathrm{mmol})$, and $\mathrm{Na}_{2} \mathrm{CO}_{3}(84$ $\mathrm{mg}, 0.80 \mathrm{mmol}$ ) were added and the tube was evacuated and backfilled with nitrogen three times after which dry DMA $(2 \mathrm{~mL})$ was added via syringe. The reaction mixture was stirred at $130{ }^{\circ} \mathrm{C}$ for $24 \mathrm{~h}$. Upon completion, the reaction tube was cooled to room temperature. The solution was diluted with dichloromethane $(30 \mathrm{~mL})$ and filtered through a pad of celite. The celite was then washed with dichloromethane $(2 \times 30 \mathrm{~mL})$. The solvent was removed by rotary evaporation (to $\sim 2 \mathrm{~mL}$ ) and concentrated under vacuum. The resulting residue was purified by silica gel column chromatography (gradient elution, dichloromethane:ethyl acetate $(19: 1, \mathrm{v} / \mathrm{v})$ to $(7: 1, \mathrm{v} / \mathrm{v}))$, to yield the corresponding product. 
Procedure B: An oven-dried $50 \mathrm{~mL}$ Schlenk tube with a stirring bar was charged with carboxylic acid 4 (0.400 mmol), $\mathrm{Na}_{2} \mathrm{CO}_{3}(42.3 \mathrm{mg}, 0.400 \mathrm{mmol})$, and dry DMA (1 mL). The reaction vessel was placed in a pre-heated oil bath and stirred for $0.5 \mathrm{~h}$ at $110{ }^{\circ} \mathrm{C}$. The solvent was then removed under reduced pressure until dry and the carboxylate salt was used without further purification. The corresponding benzamide $1(0.200 \mathrm{mmol})$, $\mathrm{Ni}(\mathrm{OAc})_{2} \bullet 4 \mathrm{H}_{2} \mathrm{O}(9.9 \mathrm{mg}, 0.040 \mathrm{mmol}), \mathrm{AgNO}_{3}(136 \mathrm{mg}, 0.800 \mathrm{mmol})$, and $\mathrm{Na}_{2} \mathrm{CO}_{3}(84$ $\mathrm{mg}, 0.80 \mathrm{mmol}$ ) were added and the tube was evacuated and backfilled with nitrogen three times after which DMA (2 $\mathrm{mL})$ was added via syringe. The reaction mixture was stirred at $170{ }^{\circ} \mathrm{C}$ for $24 \mathrm{~h}$. Upon completion, the reaction tube was cooled to room temperature. The solution was diluted with dichloromethane $(30 \mathrm{~mL})$ and filtered through a pad of celite, the celite was washed with dichloromethane $(2 \times 30 \mathrm{~mL})$. The solvent was removed by rotary evaporation (to $\sim 2 \mathrm{~mL}$ ) and concentrated under vacuo. The residue was purified by silica gel column chromatography (gradient elution, dichloromethane:ethyl acetate $(19: 1, \mathrm{v} / \mathrm{v})$ to $(7: 1, \mathrm{v} / \mathrm{v}))$, yielding the corresponding product.

Procedure C: An oven-dried $50 \mathrm{~mL}$ Schlenk tube with a stirring bar was charged with carboxylic acid 4 (0.400 mmol), $\mathrm{Na}_{2} \mathrm{CO}_{3}(42.3 \mathrm{mg}, 0.400 \mathrm{mmol})$, and dry DMA (1 mL). The reaction vessel was placed in a pre-heated oil bath and stirred for $0.5 \mathrm{~h}$ at $110{ }^{\circ} \mathrm{C}$. The solvent was removed under reduced pressure until dry and the carboxylate salt was used without further purification. The corresponding benzamide 1 (0.200 mmol), $\mathrm{Ni}(\mathrm{OAc})_{2} \bullet 4 \mathrm{H}_{2} \mathrm{O}(9.9 \mathrm{mg}, 0.040 \mathrm{mmol}), \mathrm{AgNO}_{3}(136 \mathrm{mg}, 0.800 \mathrm{mmol}), \mathrm{Na}_{2} \mathrm{CO}_{3}(84 \mathrm{mg}$, $0.80 \mathrm{mmol}$ ) were added and the tube was evacuated and backfilled with nitrogen three times after which DMA $(2 \mathrm{~mL})$ was added via syringe. The reaction mixture was stirred at $130{ }^{\circ} \mathrm{C}$ for $8 \mathrm{~h}$ then $170{ }^{\circ} \mathrm{C}$ for $16 \mathrm{~h}$. Upon completion, the reaction tube was cooled to room temperature. The solution was diluted with dichloromethane $(30 \mathrm{~mL})$ and filtered through a pad of celite, the celite was washed with dichloromethane $(2 \times 30 \mathrm{~mL})$. The solvent was removed by rotary evaporation (to $\sim 2 \mathrm{~mL}$ ) and concentrated under vacuo. The residue was purified by silica gel column chromatography (gradient elution, 
dichloromethane:ethyl acetate $(19: 1, \mathrm{v} / \mathrm{v})$ to $(7: 1, \mathrm{v} / \mathrm{v}))$, yielding the corresponding product.

Procedure for the $1 \mathrm{mmol}$ Scale Oxidative Decarboxylative Annulation Reaction. An oven-dried $100 \mathrm{~mL}$ Schlenk tube with a stirring bar was charged with 2fluoronicotinic acid 4a (282 mg, $2.00 \mathrm{mmol}), \mathrm{Na}_{2} \mathrm{CO}_{3}(212 \mathrm{mg}, 2.00 \mathrm{mmol})$, and dry DMA ( $5 \mathrm{~mL})$. The reaction vessel was placed in a pre-heated oil bath and stirred for $0.5 \mathrm{~h}$ at $110^{\circ} \mathrm{C}$. The solvent was removed under reduced pressure until dry and the carboxylate salt was used without further purification. The corresponding 2-methyl-N-(quinolin-8-yl) benzamide 1a (262 mg, $1.00 \mathrm{mmol}), \mathrm{Ni}(\mathrm{OAc})_{2} \bullet 4 \mathrm{H}_{2} \mathrm{O}$ (49.7 mg, $\left.0.20 \mathrm{mmol}\right), \mathrm{AgNO}_{3}(679$ $\mathrm{mg}, 4.00 \mathrm{mmol}), \mathrm{Na}_{2} \mathrm{CO}_{3}(424 \mathrm{mg}, 4.00 \mathrm{mmol})$ were added and the tube was evacuated and backfilled with nitrogen three times after which DMA $(10 \mathrm{~mL})$ was added via syringe. The reaction mixture was stirred at $130{ }^{\circ} \mathrm{C}$ for $24 \mathrm{~h}$. Upon completion, the reaction tube was cooled to room temperature. The solution was diluted with dichloromethane $(50 \mathrm{~mL})$ and filtered through a pad of celite, the celite was washed with dichloromethane $(4 \times 50 \mathrm{~mL}$ ). The solvent was removed by rotary evaporation (to $\sim 10$ $\mathrm{mL}$ ) and concentrated under vacuo. The residue was purified by silica gel column chromatography (gradient elution, dichloromethane:ethyl acetate (19:1, v/v) to $(7: 1$, $\mathrm{v} / \mathrm{v}))$, yielding $253 \mathrm{mg}(0.75 \mathrm{mmol})$ of the corresponding product in $75 \%$ yield.

\section{Challenging Carboxylates}

Heteroaromatic carboxylates that do not undergo decarboxylative arylation and cyclization under the standard reaction conditions.
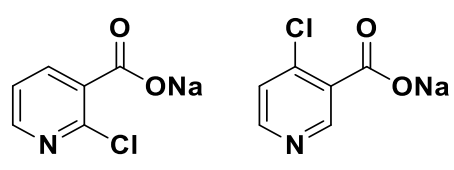<smiles>O=C(O[Na])c1ccncc1F</smiles><smiles>O=C(O[Na])c1ccnc(F)c1F</smiles><smiles>O=C(O[N+](=O)[O-])c1ccncc1[N+](=O)[O-]</smiles> 


\section{Mechanistic Studies}

\section{Control Reactions with Radical Scavengers.}

An oven-dried $50 \mathrm{~mL}$ Schlenk tube with a stirring bar was charged with 2-fluoronicotinic acid $4 \mathbf{a}(56.4 \mathrm{mg}, 0.400 \mathrm{mmol})$, sodium carbonate $(42.3 \mathrm{mg}, 0.400 \mathrm{mmol})$, and dry DMA $(1 \mathrm{~mL})$. The reaction vessel was placed in a pre-heated oil bath and stirred for $0.5 \mathrm{~h}$ at $110{ }^{\circ} \mathrm{C}$. The solvent was removed under reduced pressure until dry and the resulting carboxylate salt was used without further purification. 2-methyl- $N$-(quinolin-8-yl) benzamide 1a $(52.4 \mathrm{mg}, 0.200 \mathrm{mmol}), \mathrm{Ni}(\mathrm{OAc})_{2} \bullet 4 \mathrm{H}_{2} \mathrm{O}(9.9 \mathrm{mg}, 0.040 \mathrm{mmol}), \mathrm{AgNO}_{3}$ (136 mg, $0.800 \mathrm{mmol}), \mathrm{Na}_{2} \mathrm{CO}_{3}(84 \mathrm{mg}, 0.80 \mathrm{mmol})$, and either TEMPO (31.2 mg, 0.200 mmol) or DHA $(36.0,0.200 \mathrm{mmol})$ were added. The tube was evacuated and backfilled with nitrogen three times after which DMA $(2 \mathrm{~mL})$ was added via syringe. The reaction mixture was stirred at $130{ }^{\circ} \mathrm{C}$ for $24 \mathrm{~h}$. Upon completion, the reaction tube was cooled to room temperature. The solution was diluted with dichloromethane $(30 \mathrm{~mL})$ and filtered through a pad of celite. The celite was then washed with dichloromethane $(2 \times 30 \mathrm{~mL})$. The solvent was concentrated by rotary evaporation (to $\sim 2 \mathrm{~mL}$ ) and the remaining solvent was removed under vacuum. A 1,3,5-trimethoxybenzene $(5.00 \mathrm{mg})$ NMR standard was added to the residue and the crude mixture was dissolved in $\mathrm{CDCl}_{3}$ for ${ }^{1} \mathrm{H} \mathrm{NMR}$ analysis. 


\section{Control Reactions with Radical Scavengers ${ }^{a}$}<smiles>Cc1ccccc1C(=O)Nc1cccc2cccnc12</smiles>

$1 \mathrm{a}$

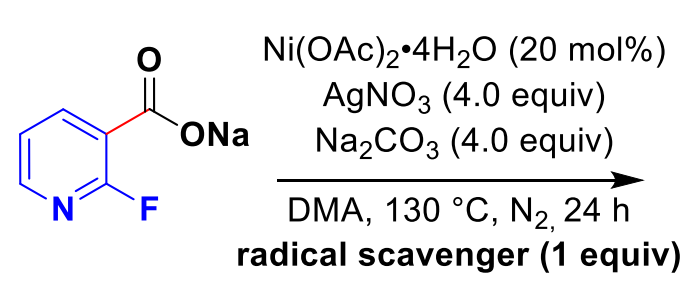

4a<smiles>Cc1cccc2c1c(=O)n(-c1cccc3cccnc13)c1ncccc21</smiles>

$5 a$

\begin{tabular}{lll}
\hline entry & radical scavenger (1 equiv) & yield 5a $(\%)^{b}$ \\
\hline 1 & none & 81 \\
2 & TEMPO & 74 \\
3 & DHA & 78
\end{tabular}

${ }^{a}$ Reaction conditions: 1a $(0.200 \mathrm{mmol}), \mathbf{4 a}(0.400 \mathrm{mmol})$ in DMA $(2 \mathrm{~mL}) .{ }^{b 1} \mathrm{H} \mathrm{NMR}$ yield with 1,3,5-trimethoxybenzene as an internal standard.

\section{Kinetic Isotope Effect Experiments}

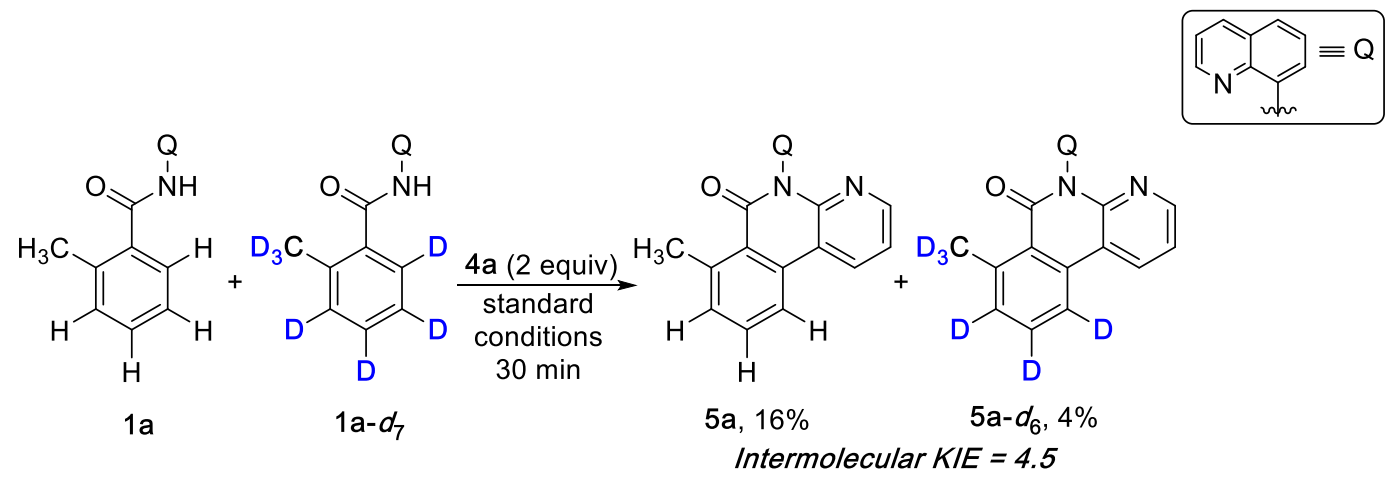

Procedure for the Intermolecular Competition Experiment. An oven-dried $50 \mathrm{~mL}$ Schlenk tube with a stirring bar was charged with 2-fluoronicotinic acid 4a $(56.4 \mathrm{mg}$, $0.400 \mathrm{mmol})$, sodium carbonate $(42.3 \mathrm{mg}, 0.400 \mathrm{mmol})$, and dry DMA (1 mL). The reaction vessel was placed in a pre-heated oil bath and stirred for $0.5 \mathrm{~h}$ at $110{ }^{\circ} \mathrm{C}$. The solvent was removed under vacuum until dry and the resulting carboxylate salt was used without further purification. 1a $(26.2 \mathrm{mg}, 0.100 \mathrm{mmol}), 6$-(methyl- $\left.d_{3}\right)-N-8$-quinolinylbenzamide-2,3,4,5- $d_{4} \mathbf{1 a -} \boldsymbol{d}_{7}(26.9 \mathrm{mg}, 0.100 \mathrm{mmol}), \mathrm{Ni}(\mathrm{OAc})_{2} \bullet 4 \mathrm{H}_{2} \mathrm{O}(9.9 \mathrm{mg}, 0.040$ $\mathrm{mmol}), \mathrm{AgNO}_{3}(136 \mathrm{mg}, 0.800 \mathrm{mmol}), \mathrm{Na}_{2} \mathrm{CO}_{3}(84 \mathrm{mg}, 0.80 \mathrm{mmol})$ were added, the tube 
was evacuated and backfilled with nitrogen three times after which DMA (2 mL) was added via syringe. The reaction mixture was stirred at $130{ }^{\circ} \mathrm{C}$ for 30 minutes. Upon completion, the reaction tube was cooled to room temperature. The solution was diluted with dichloromethane $(30 \mathrm{~mL})$ and filtered through a pad of celite. The celite was then washed with dichloromethane $(2 \times 30 \mathrm{~mL})$. The solvent was concentrated by rotary evaporation (to $\sim 2 \mathrm{~mL}$ ) and the remaining solvent was removed under vacuum. The resulting residue was purified by silica gel column chromatography (dichloromethane:ethyl acetate $(19: 1, \mathrm{v} / \mathrm{v})$ to $(7: 1, \mathrm{v} / \mathrm{v}))$, yielding the corresponding product as a mixture of $\mathbf{5 a}$ and $\mathbf{5 a}-\boldsymbol{d}_{\mathbf{6}}$ in $16 \%$ and $4 \%$ yields respectively (as determined by ${ }^{1} \mathrm{H}$ NMR spectroscopy), giving a KIE of 4.5.

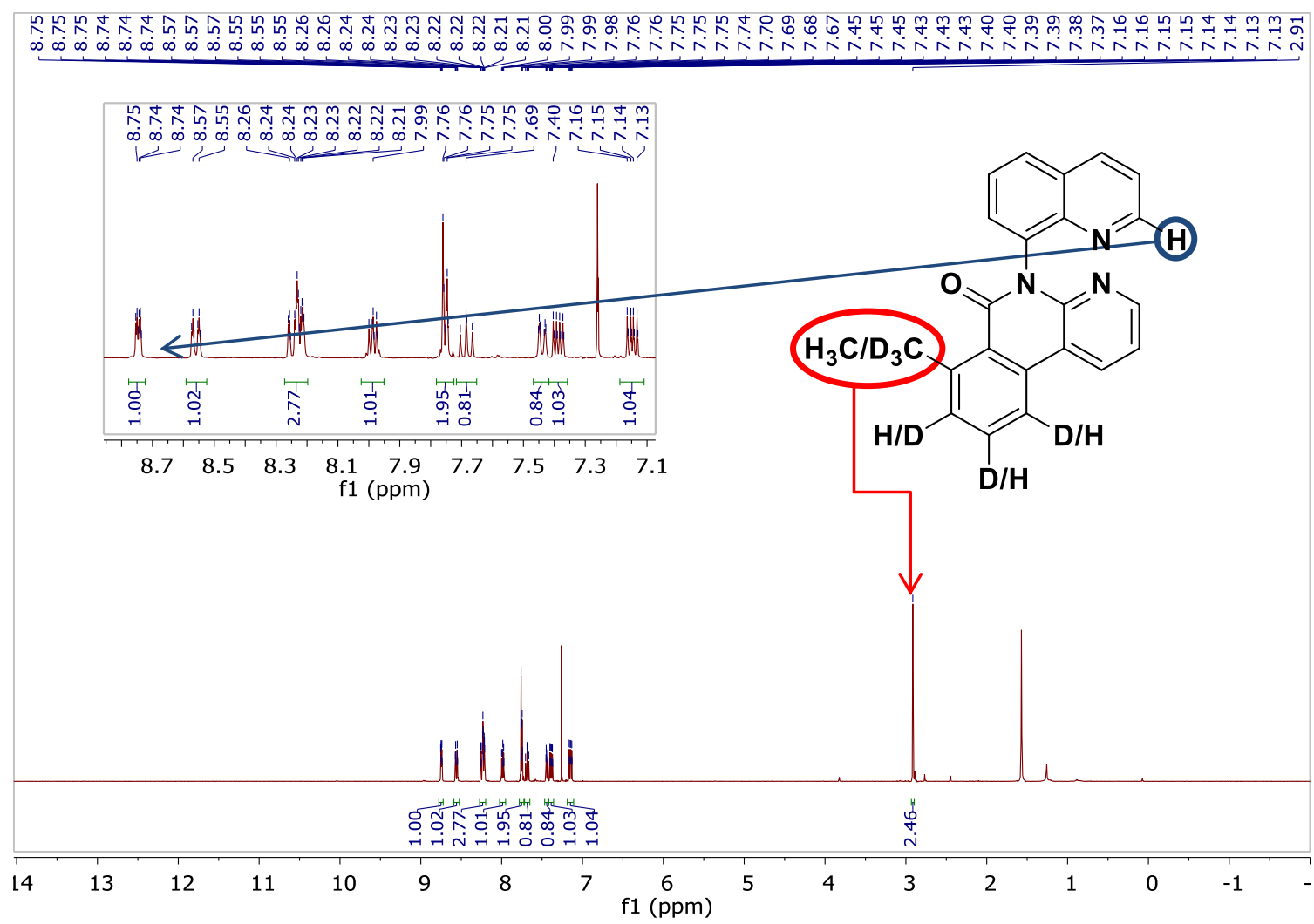




\section{Isotopic Exchange Experiment}<smiles>[2H]c1c([2H])c([2H])c(C(=O)NO)c(C(=O)O)c1[2H]</smiles>

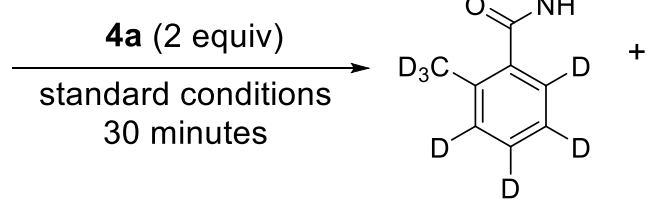

$5 \mathrm{a}-d_{7}, 75 \%,<1 \% \mathrm{H}$

N $\equiv$

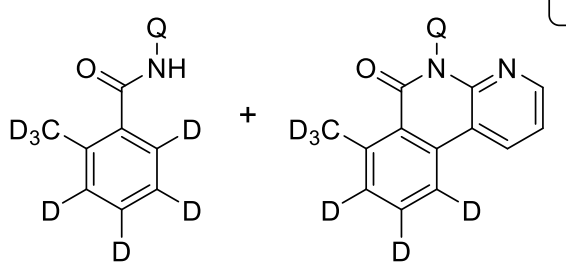

$5 a-d_{6}, 12 \%$

Procedure for the Isotopic Exchange Experiment. An oven-dried $50 \mathrm{~mL}$ Schlenk tube with a stirring bar was charged with 2-fluoronicotinic acid 4a $(56.4 \mathrm{mg}, 0.400 \mathrm{mmol})$, sodium carbonate $(42.3 \mathrm{mg}, 0.400 \mathrm{mmol})$, and dry DMA (1 mL). The reaction vessel was placed in a pre-heated oil bath and stirred for $0.5 \mathrm{~h}$ at $110{ }^{\circ} \mathrm{C}$. The solvent was removed under reduced pressure until dry and the resulting carboxylate salt was used without further purification. 1a- $\boldsymbol{d}_{7}(53.8 \mathrm{mg}, 0.200 \mathrm{mmol}), \mathrm{Ni}(\mathrm{OAc})_{2} \bullet 4 \mathrm{H}_{2} \mathrm{O}(9.9 \mathrm{mg}, 0.040 \mathrm{mmol})$, $\mathrm{AgNO}_{3}(136 \mathrm{mg}, 0.800 \mathrm{mmol})$, and $\mathrm{Na}_{2} \mathrm{CO}_{3}(84 \mathrm{mg}, 0.80 \mathrm{mmol})$ were added, the tube was evacuated and backfilled with nitrogen three times after which DMA (2 mL) was added via syringe. The reaction mixture was stirred at $130{ }^{\circ} \mathrm{C}$ for 30 minutes. Upon completion, the reaction tube was cooled to room temperature. The solution was diluted with dichloromethane $(30 \mathrm{~mL})$ and filtered through a pad of celite. The celite was then washed with dichloromethane $(2 \times 30 \mathrm{~mL})$. The solvent was concentrated by rotary evaporation (to $\sim 2 \mathrm{~mL}$ ) and the remaining solvent removed under vacuum. The residue was purified by silica gel column chromatography (gradient elution, hexanes:ethyl acetate $(9: 1, \mathrm{v} / \mathrm{v})$ to $(4: 1, \mathrm{v} / \mathrm{v}))$, yielding the corresponding product. The extent of proton incorporation was determined to be $0.01 \mathrm{H}$, less than $1 \%$, by ${ }^{1} \mathrm{H}$ NMR spectroscopy. 


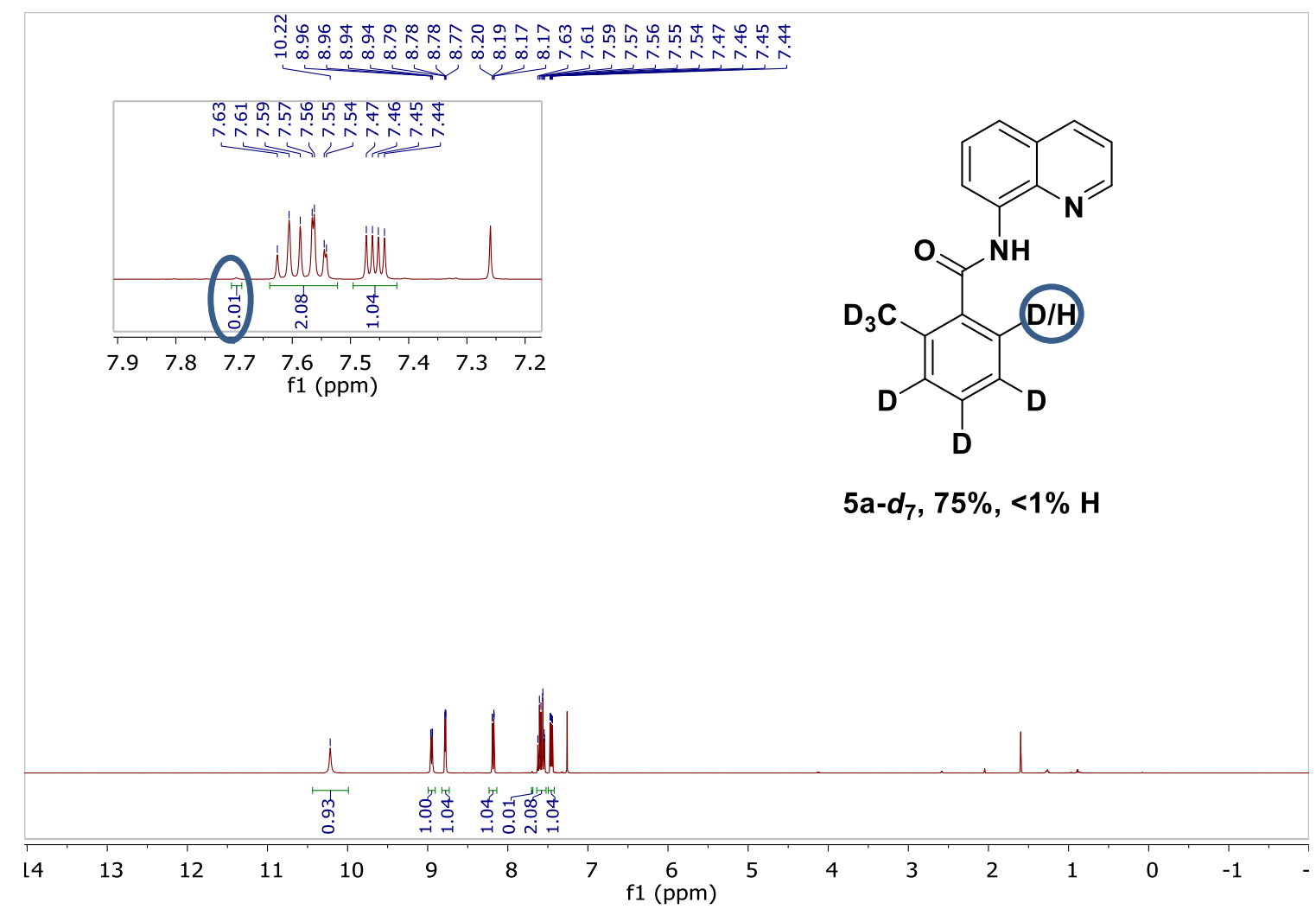

General Procedure for the Synthesis of (Hetero)Aryl Benzamides (1)

Procedure A: Synthesis from the Acid Chloride. A $100 \mathrm{~mL}$ two-neck round bottom flask was charged with 8-aminoquinoline $(1.44 \mathrm{~g}, 10.0 \mathrm{mmol}), \mathrm{Et}_{3} \mathrm{~N}(1.53 \mathrm{~mL}, 11.0$ $\mathrm{mmol})$, and methylene chloride $(20 \mathrm{~mL})$ under a $\mathrm{N}_{2}$ atmosphere. The corresponding acid chloride $(12.0 \mathrm{mmol})$ was added dropwise at room temperature over 10 minutes. The mixture was stirred for $6 \mathrm{~h}$ at room temperature. The reaction was quenched with $10 \mathrm{~mL}$ of saturated $\mathrm{NaHCO}_{3}$ and extracted with methylene chloride $(3 \times 25 \mathrm{~mL})$. The combined organic layer was washed with brine $(15 \mathrm{~mL})$ and dried over anhydrous $\mathrm{Na}_{2} \mathrm{SO}_{4}$ and filtered. The solvent was removed under reduced pressure and the residue was purified via silica gel column chromatography (gradient elution, hexanes : ethyl acetate $(15: 1, \mathrm{v} / \mathrm{v})$ to $(1: 1, \mathrm{v} / \mathrm{v}))$.

Procedure B: Synthesis from the carboxylic acid. A $50 \mathrm{~mL}$ two-neck round bottom flask was charged with the corresponding carboxylic acid $(1.70 \mathrm{mmol})$, anhydrous $\mathrm{N}, \mathrm{N}$ dimethylformamide ( 2 drops), and methylene chloride $(2 \mathrm{~mL})$ under a $\mathrm{N}_{2}$ atmosphere at 0 
${ }^{\circ} \mathrm{C}$. Oxalyl chloride $(0.17 \mathrm{~mL}, 2.00 \mathrm{mmol})$ was then added dropwise and the reaction mixture was allowed to stir for $3 \mathrm{~h}$ at room temperature. The solvent was removed under reduced pressure and the resulting acid chloride was used without further purification. A $100 \mathrm{~mL}$ two-neck round bottom flask was charged with 8-aminoquinoline (255 mg, 1.70 mmol), $\mathrm{Et}_{3} \mathrm{~N}(0.278 \mathrm{~mL}, 2.00 \mathrm{mmol})$, and methylene chloride $(10 \mathrm{~mL})$ under a $\mathrm{N}_{2}$ atmosphere at $0{ }^{\circ} \mathrm{C}$. The corresponding acid chloride was added dropwise to the reaction and stirred for $3 \mathrm{~h}$. The reaction was quenched with $5 \mathrm{~mL}$ of saturated $\mathrm{NaHCO}_{3}$ and extracted with methylene chloride three times $(3 \times 25 \mathrm{~mL})$. The combined organic layer was washed with brine $(15 \mathrm{~mL})$ and dried over anhydrous $\mathrm{Na}_{2} \mathrm{SO}_{4}$ and filtered. The solvent was removed under reduced pressure and the residue was purified via silica gel column chromatography (gradient elution, hexanes : ethyl acetate $(19: 1, \mathrm{v} / \mathrm{v})$ to $(9: 1$, $\mathrm{v} / \mathrm{v}))$.

\section{Base Promoted Cyclization Reaction}
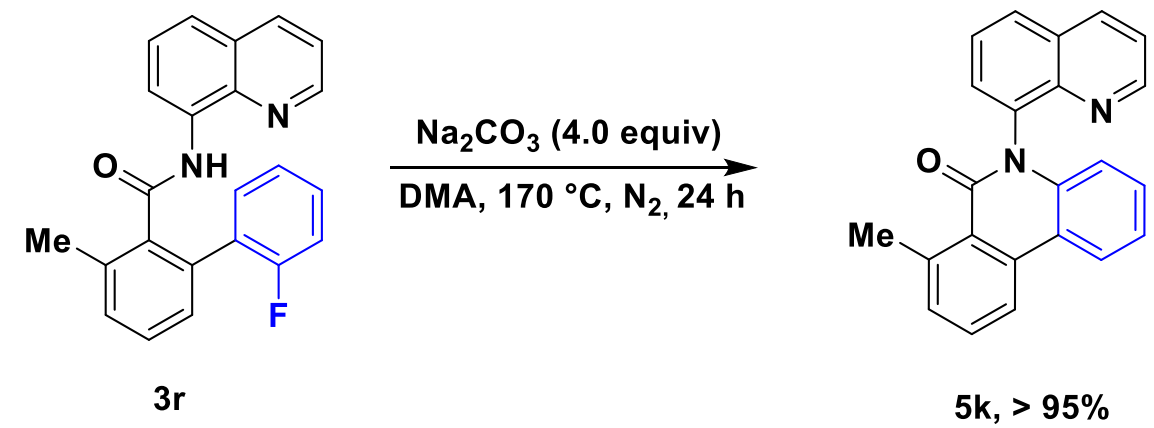

An oven-dried $50 \mathrm{~mL}$ Schlenk tube with a stirring bar was charged with 2'-fluoro-3methyl- $N$-(quinolin-8-yl)-[1,1'-biphenyl]-2-carboxamide ${ }^{66}(71.2 \mathrm{mg}, 0.200 \mathrm{mmol})$ and $\mathrm{Na}_{2} \mathrm{CO}_{3}(84 \mathrm{mg}, 0.80 \mathrm{mmol})$. The tube was evacuated and backfilled with nitrogen three times after which DMA (2 mL) was added via syringe. The reaction mixture was stirred at $170{ }^{\circ} \mathrm{C}$ for $24 \mathrm{~h}$. Upon completion, the reaction tube was cooled to room temperature. The solution was diluted with dichloromethane $(30 \mathrm{~mL})$ and filtered through a pad of celite, the celite was washed with dichloromethane $(2 \times 30 \mathrm{~mL})$. The solvent was concentrated by rotary evaporation (to $\sim 2 \mathrm{~mL}$ ) and the remaining solvent was removed under vacuum. Then 1,3,5-trimethoxybenzene $(5.00 \mathrm{mg})$ was added to the residue and the crude mixture was dissolved in $\mathrm{CDCl}_{3}$ for analysis by ${ }^{1} \mathrm{H}$ NMR spectroscopy. 


\section{General Procedures for the Synthesis of Fluorinated Carboxylic Acids (4)}

\section{Procedure A:Synthesis from Silver(II) Fluoride}

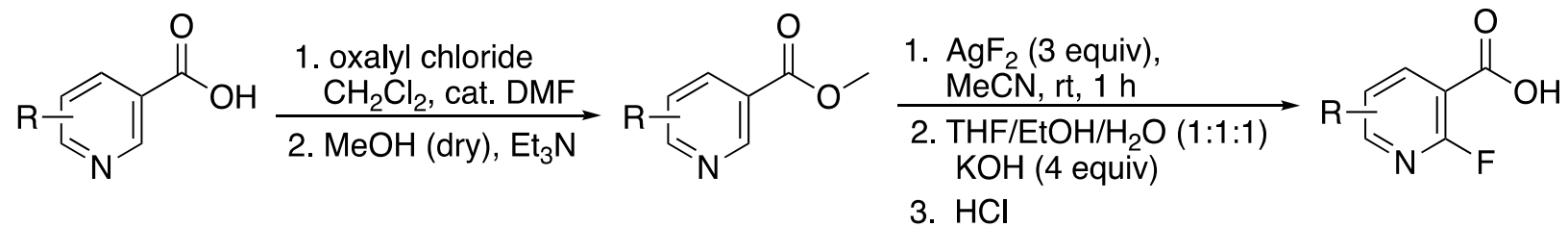

Synthesis of methyl esters. A $50 \mathrm{~mL}$ two-neck round bottom flask was charged with the corresponding carboxylic acid (3.62 mmol), N,N-dimethylformamide (3-4 drops), and methylene chloride $(4 \mathrm{~mL})$ under a $\mathrm{N}_{2}$ atmosphere at $0{ }^{\circ} \mathrm{C}$. Oxalyl chloride $(0.32 \mathrm{~mL}, 3.8$ mmol) was then added dropwise and the reaction mixture was allowed to stir for $3 \mathrm{~h}$ at room temperature after removal of the ice bath. The solvent was removed under reduced pressure and anhydrous methanol was then added to the resulting crude acid chloride. Triethylamine $(0.55 \mathrm{~mL}, 4.00 \mathrm{mmol})$ was added dropwise to the mixture and the reaction was allowed to stir for $3 \mathrm{~h}$. The reaction was quenched with saturated $\mathrm{NaHCO}_{3}(5 \mathrm{~mL})$ and extracted with dichloromethane $(3 \times 20 \mathrm{~mL})$. The combined organic layers were washed with brine $(10 \mathrm{~mL})$ and dried over anhydrous $\mathrm{Na}_{2} \mathrm{SO}_{4}$ and filtered. The solvent was removed under reduced pressure and the residue was purified via silica gel column chromatography (gradient elution, hexanes : ethyl acetate $(19: 1, \mathrm{v} / \mathrm{v})$ to $(9: 1, \mathrm{v} / \mathrm{v})$ or (gradient elution, hexanes : dichloromethane $(15: 1, \mathrm{v} / \mathrm{v})$ to $(1: 1, \mathrm{v} / \mathrm{v})$.

Fluorination of heteroaryl methyl esters. The title compounds were prepared according to a literature procedure. ${ }^{67}$ In a $\mathrm{N}_{2}$ filled glovebox, the pyridine substrate $(1.00 \mathrm{mmol})$ and anhydrous MeCN (20 mL) were combined in an oven-dried round bottom flask. While rapidly stirring, $\mathrm{AgF}_{2}(438 \mathrm{mg}, 3.00 \mathrm{mmol})$ was added at once. The round bottom flask was capped with a rubber septum and stirred for 1 hour. The reaction mixture was then removed from the glovebox and poured into a separatory funnel containing $40 \mathrm{~mL}$ of saturated aqueous $\mathrm{NaHCO}_{3}$ and the mixture extracted with $\mathrm{Et}_{2} \mathrm{O}(2 \times 30 \mathrm{~mL}$ ). The organic layer was washed with brine $(40 \mathrm{~mL})$ and dried over anhydrous $\mathrm{Na}_{2} \mathrm{SO}_{4}$ and filtered. The solvent was removed under reduced pressure and the residue was purified via silica gel column chromatography. 
Hydrolysis of fluorinated heteroaryl methyl esters. A $100 \mathrm{~mL}$ round bottom flask was charged with the fluorinated heteroaryl methyl ester (1.0 mmol), THF/EtOH/ $\mathrm{H}_{2} \mathrm{O}(1: 1: 1$, $2 \mathrm{~mL} / 2 \mathrm{~mL} / 2 \mathrm{~mL}$ ), and $\mathrm{KOH}$ (4 equiv). The mixture was monitored by TLC until all starting material was consumed. The reaction was acidified with $\mathrm{HCl}(2 \mathrm{~N})$ until a $\mathrm{pH}$ of 2 was achieved. The reaction mixture was poured into a separatory funnel and extracted with ethyl acetate $(3 \times 50 \mathrm{~mL})$ and dried over anhydrous $\mathrm{Na}_{2} \mathrm{SO}_{4}$ and filtered. The solvent was removed under reduced pressure and the resulting acid was used without further purification.

\section{Procedure B: Synthesis from Lithiation and Fluorination with NFSI}

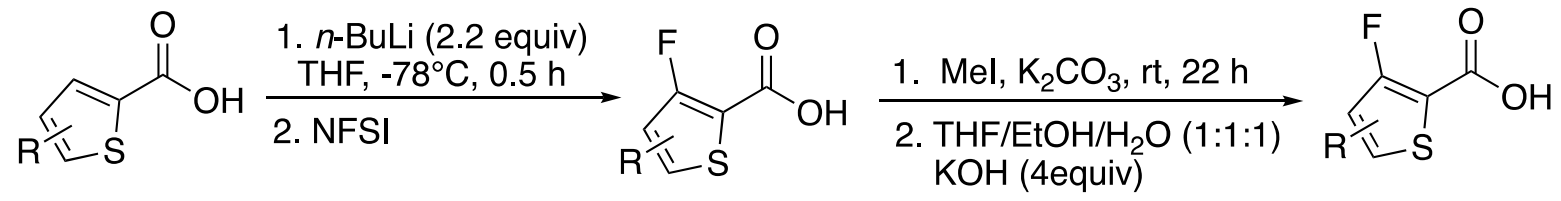

3. $\mathrm{HCl}$

Fluorination of 2-thiophenecarboxylic acid substrates. The title compounds were prepared following a procedure modified from the literature. ${ }^{68}$ To a pre-cooled $\left(-78{ }^{\circ} \mathrm{C}\right)$ solution of 2-thiophenecarboxylic acid substrate $(5.61 \mathrm{mmol})$ in anhydrous THF $(25 \mathrm{~mL})$ was added $n$-BuLi $(4.93 \mathrm{~mL}, 12.3 \mathrm{mmol})$ dropwise. After the reaction stirred for 30 minutes, a solution of $N$-fluorobenzenesulfonimide (NFSI, $2.65 \mathrm{~g}, 8.41 \mathrm{mmol}$ ) in anhydrous THF $(22 \mathrm{~mL})$ was added dropwise at $-78^{\circ} \mathrm{C}$. The solution was stirred at $-78^{\circ} \mathrm{C}$ for 5 hours and then warmed slowly and allowed to stir at room temperature overnight. The reaction was quenched with $6 \mathrm{~N} \mathrm{HCl}(3 \mathrm{~mL})$ at $0{ }^{\circ} \mathrm{C}$. The reaction mixture was poured into a separatory funnel and extracted with ethyl acetate $(3 \times 75 \mathrm{~mL})$ and dried over anhydrous $\mathrm{Na}_{2} \mathrm{SO}_{4}$. The solvent was removed under reduced pressure. The crude solid was then dissolved in a mixture of a minimum amount of water $(\sim 150 \mathrm{~mL})$ and $\mathrm{NaOH}$ (1.2 equiv). The aqueous layer was poured into a separatory funnel and washed with dichloromethane until all residual NFSI was removed. The aqueous layer was then acidified with $\mathrm{HCl}(2 \mathrm{~N})$ until a $\mathrm{pH}$ of 2 was achieved. The reaction mixture was poured into a separatory funnel and extracted with ethyl acetate $(3 \times 75 \mathrm{~mL})$ and dried over 
anhydrous $\mathrm{Na}_{2} \mathrm{SO}_{4}$ and filtered. The solvent was removed under reduced pressure to yield the crude fluorinated thiophenecarboxylic acid which was used as described below without further purification.

Methylation of 2-thiophenecarboxylic acid substrates. To an oven-dried round bottom flask was charged the crude fluorinated thiophenecarboxylic acid, $\mathrm{K}_{2} \mathrm{CO}_{3}(1.07 \mathrm{~g}, 7.74$ $\mathrm{mmol})$, anhydrous DMF (10 mL), and methyl iodide $(0.70 \mathrm{~mL}, 11.2 \mathrm{mmol})$. The reaction mixture was stirred at room temperature for 22 hours. After completion, saturated ammonium chloride $(15 \mathrm{~mL})$ was added and the resulting mixture was extracted with ethyl acetate $(3 \times 50 \mathrm{~mL})$. The organic phase was washed with water $(100 \mathrm{~mL})$ and brine (20 mL), dried over anhydrous $\mathrm{Na}_{2} \mathrm{SO}_{4}$, filtered, and the solvent was removed under reduced pressure. The residue was purified via silica gel column chromatography to give the corresponding methyl ester.

Hydrolysis of fluorinated methyl 2-thiophenecarboxylate. A $100 \mathrm{~mL}$ round bottom flask was charged with the fluorinated methyl 2-thiophenecarboxylate $(2.0 \mathrm{mmol})$, $\left.\mathrm{THF} / \mathrm{EtOH} / \mathrm{H}_{2} \mathrm{O}(1: 1: 1,4 \mathrm{~mL} / 4 \mathrm{~mL} / 4 \mathrm{~mL})\right)$, and $\mathrm{KOH}$ (4 equiv). The mixture was monitored by TLC until all starting material was consumed. The reaction was acidified with $\mathrm{HCl}(2 \mathrm{~N})$ until a $\mathrm{pH}$ of 2 was achieved. The reaction mixture was poured into a separatory funnel and extracted with ethyl acetate $(3 \times 50 \mathrm{~mL})$ and dried over anhydrous $\mathrm{Na}_{2} \mathrm{SO}_{4}$ and filtered. The solvent was removed under reduced pressure and used without further purification.

\section{Procedure C: Synthesis from Lithiation and Carboxylation}

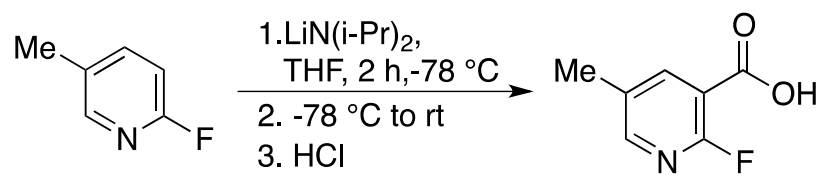

Carboxylation of 2-fluoro-5-methylpyridine: The title compound was prepared following a modification of a literature synthesis. ${ }^{69}$ A $2.5 \mathrm{M}$ solution of $n$-butyl lithium in hexanes $(2.28 \mathrm{~mL}, 5.70 \mathrm{mmol})$ was added dropwise to a solution of freshly distilled 
diisopropylamine $(0.80 \mathrm{~mL}, 5.70 \mathrm{mmol})$ in THF $(10 \mathrm{~mL})$ at $-78{ }^{\circ} \mathrm{C}$. After complete addition, the mixture was allowed to warm to $0{ }^{\circ} \mathrm{C}$ and stir at this temperature for 10 minutes. The solution was then cooled back down to $-78{ }^{\circ} \mathrm{C}$ and a solution of 2-fluoro-5methylpyridine $(0.59 \mathrm{~mL}, 5.70 \mathrm{mmol})$ in THF $(1.85 \mathrm{~mL})$ was added dropwise. The reaction mixture was stirred at $-78{ }^{\circ} \mathrm{C}$ for 2 hours and then quenched with freshly made dry ice ( 1 brick). The reaction mixture was allowed to warm to room temperature and was then acidified with $\mathrm{HCl}(2 \mathrm{~N})$ until a $\mathrm{pH}$ of 2 was achieved and extracted with ethyl acetate $(3 \times 50 \mathrm{~mL})$. The organic phase was washed with brine $(20 \mathrm{~mL})$ and dried over anhydrous $\mathrm{Na}_{2} \mathrm{SO}_{4}$, filtered, and the solvent was removed under reduced pressure.

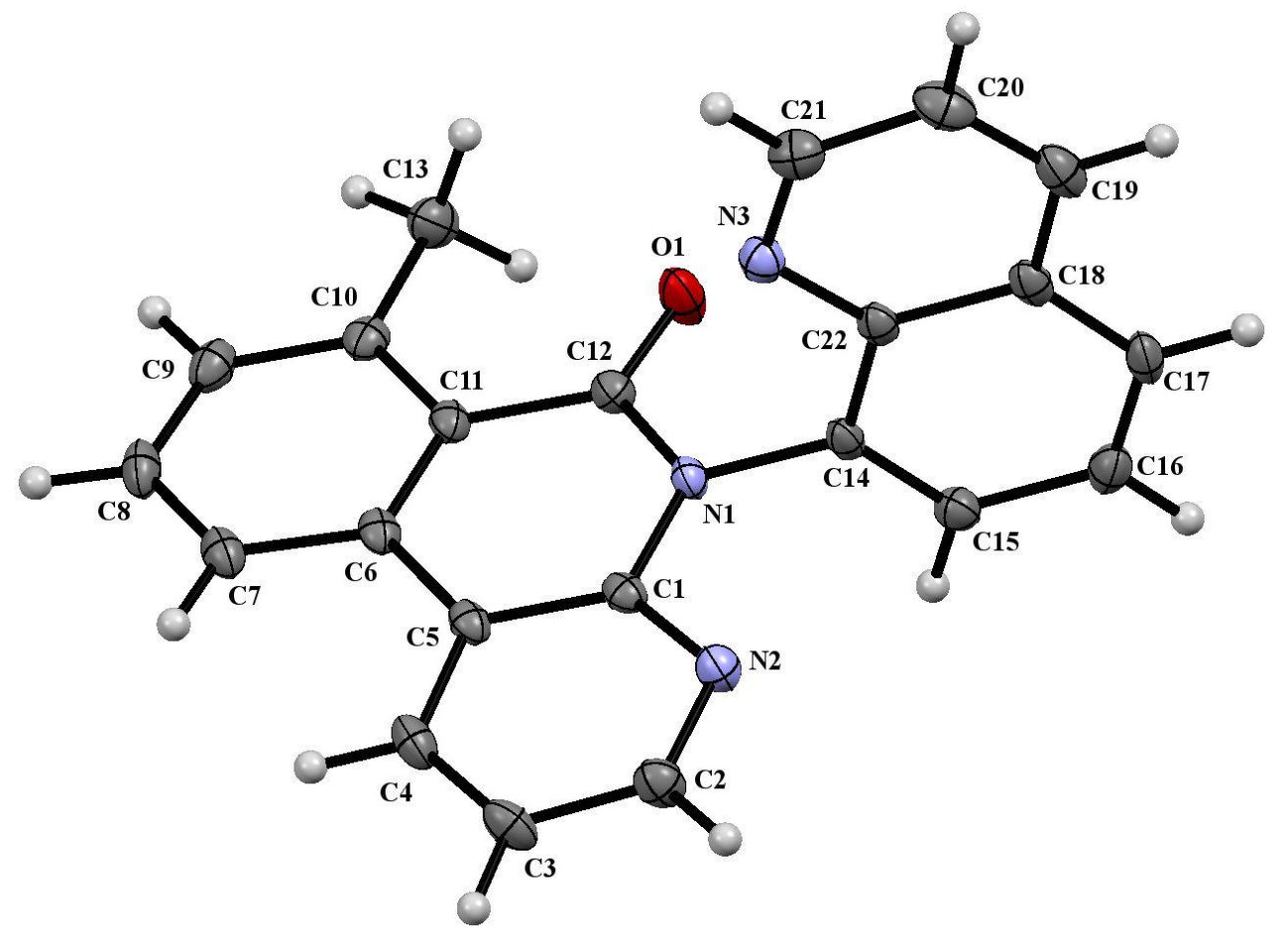

Perspective view of the molecular structure of the $\mathrm{C}_{22} \mathrm{H}_{15} \mathrm{~N}_{3} \mathrm{O}$ (5a) with the atom labeling scheme for the non-hydrogen atoms. Thermal ellipsoids are scaled to enclose $50 \%$ probability.

\section{Description of the X-ray Structural Analysis of $\mathrm{C}_{22} \mathrm{H}_{15} \mathrm{~N}_{3} \mathrm{O}(5 \mathrm{a})$.}

A colorless rectangular crystal of $\mathrm{C}_{22} \mathrm{H}_{15} \mathrm{~N}_{3} \mathrm{O}$ (5a) was covered in a polybutene oil (Sigma-Aldrich) and placed on the end of a MiTeGen loop. The sample was cooled to 
$100 \mathrm{~K}$ with an Oxford Cryostream 700 system and optically aligned on a Bruker AXS D8 Venture fixed-chi X-ray diffractometer equipped with a Triumph monochromator, a Mo K $\alpha$ radiation source $(\lambda=0.71073 \AA)$, and a PHOTON 100 CMOS detector. Three sets of 12 frames each were collected using the omega scan method with a 10 s exposure time. Integration of these frames followed by reflection indexing and least-squares refinement produced a crystal orientation matrix for the monoclinic crystal lattice that was used for the structural analysis.

Data collection consisted of the measurement of a total of 246 frames in two runs using omega scans with the detector held at $5.00 \mathrm{~cm}$ from the crystal. Frame scan parameters are summarized in Table 1 below:

Data collection details for $\mathrm{C}_{22} \mathrm{H}_{15} \mathrm{~N}_{3} \mathrm{O}(5 \mathrm{a})$.

\begin{tabular}{|c|c|c|c|c|c|c|c|}
\hline Run & $2 \theta$ & $\omega$ & $\varphi$ & $\chi$ & Scan Width $\left(^{\circ}\right)$ & Frames & $\begin{array}{c}\text { Exposure } \\
\text { Time }(\mathrm{sec})\end{array}$ \\
\hline 1 & 17.45 & -164.74 & -217.75 & 54.78 & 1.50 & 123 & 40.00 \\
\hline 2 & 17.45 & -164.74 & 45.40 & 54.78 & 1.50 & 123 & 40.00 \\
\hline
\end{tabular}

The APEX3 software program (version 2016.9-0) ${ }^{100}$ was used for diffractometer control, preliminary frame scans, indexing, orientation matrix calculations, least-squares refinement of cell parameters, and the data collection. The frames were integrated with the Bruker SAINT software package using a narrow-frame algorithm. The integration of the data using a monoclinic unit cell yielded a total of 14343 reflections to a maximum $\theta$ angle of $27.50^{\circ}(0.77 \AA$ resolution), of which 3597 were independent (average redundancy 3.987, completeness $\left.=99.8 \%, \mathrm{R}_{\mathrm{int}}=3.31 \%, \mathrm{R}_{\mathrm{sig}}=3.09 \%\right)$ and 2792 $(77.62 \%)$ were greater than $2 \sigma\left(F^{2}\right)$. The final cell constants of $\underline{a}=13.4326(7) \AA, \underline{b}=$ 7.3348(4) $\AA, \underline{c}=17.1068(9) \AA, \beta=111.9152(15)^{\circ}$, volume $=1563.66(14) \AA^{3}$, are based 
upon the refinement of the XYZ-centroids of 6729 reflections above $20 \sigma(\mathrm{I})$ with $6.445^{\circ}$ $<2 \theta<60.15^{\circ}$. Data were corrected for absorption effects using the Multi-Scan method (SADABS). The ratio of minimum to maximum apparent transmission was 0.809 . The calculated minimum and maximum transmission coefficients (based on crystal size) are 0.962 and 0.992 .

The structure was solved by direct methods and difference Fourier analysis using the programs provided by SHELXL-2014/7. ${ }^{101}$ Idealized positions for the hydrogen atoms were included as fixed contributions using a riding model with isotropic temperature factors set at 1.2 (aromatic hydrogens) or 1.5 times (methyl hydrogens) that of the adjacent carbon atom. The positions of the methyl hydrogen atoms were optimized by a rigid rotating group refinement with idealized angles. Full-matrix least-squares refinement, based upon the minimization of $\Sigma \mathrm{w}_{\mathrm{i}}\left|\mathrm{F}_{\mathrm{o}}{ }^{2}-\mathrm{F}_{\mathrm{c}}{ }^{2}\right|^{2}$, with weighting $\mathrm{w}_{\mathrm{i}}^{-1}=$ $\left[\sigma^{2}\left(\mathrm{~F}_{\mathrm{o}}{ }^{2}\right)+(0.0524 \mathrm{P})^{2}+0.7107 \mathrm{P}\right]$, where $\mathrm{P}=\left(\operatorname{Max}\left(\mathrm{F}_{\mathrm{o}}{ }^{2}, 0\right)+2 \mathrm{~F}_{\mathrm{c}}{ }^{2}\right) / 3$. $^{101}$ The final anisotropic full-matrix least-squares refinement on $\mathrm{F}^{2}$ with 236 variables converged at $\mathrm{R} 1=3.99 \%$, for the 2792 observed data with $\mathrm{I}>2 \sigma(\mathrm{I})$ and $w \mathrm{R} 2=11.05 \%$ for all data. The value of the goodness-of-fit was 1.019. ${ }^{102}$ The linear absorption coefficient, atomic scattering factors, and anomalous dispersion corrections were calculated from values found in the International Tables of X-ray Crystallography. ${ }^{103}$

\section{Crystal data for $\mathrm{C}_{22} \mathrm{H}_{15} \mathrm{~N}_{3} \mathrm{O}(5 \mathrm{a})$.}

Ident. code jh43cms

Chemical form. $\mathrm{C}_{22} \mathrm{H}_{15} \mathrm{~N}_{3} \mathrm{O}$

Formula wght. $\quad 337.37 \mathrm{~g} / \mathrm{mol}$

Temperature $\quad 100(2) \mathrm{K}$

Wavelength $\quad 0.71073 \AA$

Crystal size $\quad 0.086 \times 0.277 \times 0.432 \mathrm{~mm}$

Crystal system monoclinic 
Space group $\quad \mathrm{P} 2{ }_{1} / \mathrm{c}$ (No. 14)

Unit cell

$$
\begin{array}{ll}
\mathrm{a}=13.4326(7) \AA & \alpha=90^{\circ} \\
\mathrm{b}=7.3348(4) \AA & \beta=111.9152(15)^{\circ} \\
\mathrm{c}=17.1068(9) \AA & \gamma=90^{\circ}
\end{array}
$$

Volume, $\AA^{3} \quad 1563.66(14)$

$\begin{array}{ll}\mathrm{Z} & 4\end{array}$

Density (calc) $\quad 1.433 \mathrm{~g} / \mathrm{cm}^{3}$

Abs. coefficient $0.090 \mathrm{~mm}^{-1}$

$\mathrm{F}(000) \quad 704$

\section{Data collection and structure refinement for $\mathrm{C}_{22} \mathrm{H}_{15} \mathrm{~N}_{3} \mathrm{O}$ (5a).}

$\begin{array}{ll}\text { Theta range } & 3.06 \text { to } 27.50^{\circ} \\ \text { Index ranges } & -17 \leq \mathrm{h} \leq 17,-9 \leq \mathrm{k} \leq 8,-17 \leq 1 \leq 22 \\ \text { Reflections } & 14343 \\ \text { Independent refls } & 3597[\mathrm{R}(\mathrm{int})=0.0331] \\ \text { Coverage } & 99.8 \%\end{array}$

Absorption correction multi-scan

Max. and min. trans. 0.992 and 0.962

Refinement method Full-matrix least-squares on $\mathrm{F}^{2}$

Refinement program SHELXL-2014/7 (Sheldrick, 2014)

Data / restraints /

parameters

3597 / 0 / 236

Goodness-of-fit on $\mathrm{F}^{2} 1.019$

Final R indices 2792 data; $\mathrm{I}>2 \sigma(\mathrm{I})$ $\mathrm{R} 1=0.0399, \mathrm{wR} 2=0.0986$

all data $\mathrm{R} 1=0.0576, \mathrm{wR} 2=0.1105$ 
Largest diff. peak and

hole

0.325 and $-0.256 \mathrm{e}^{-} / \AA^{3}$

Atomic coordinates and equivalent isotropic atomic displacement parameters $\left(\AA^{2}\right)$ for $\mathrm{C}_{22} \mathrm{H}_{15} \mathrm{~N}_{3} \mathrm{O}$ (5a). $\mathrm{U}(\mathrm{eq})$ is defined as one third of the trace of the orthogonalized $\mathrm{U}_{\mathrm{ij}}$ tensor.

$\begin{array}{ccccc} & \mathrm{x} / \mathrm{a} & \mathrm{y} / \mathrm{b} & \mathrm{z} / \mathrm{c} & \mathrm{U}(\mathrm{eq}) \\ \mathrm{O} 1 & 0.13435(8) & 0.87131(16) & 0.22197(6) & 0.0267(3) \\ \mathrm{N} 1 & 0.21961(8) & 0.76163(16) & 0.14127(7) & 0.0147(2) \\ \mathrm{N} 2 & 0.31986(9) & 0.66240(16) & 0.06551(7) & 0.0184(3) \\ \mathrm{N} 3 & 0.27078(9) & 0.45464(16) & 0.24227(7) & 0.0181(3) \\ \mathrm{C} 1 & 0.22280(10) & 0.70165(18) & 0.06493(8) & 0.0148(3) \\ \mathrm{C} 2 & 0.32566(12) & 0.6120(2) & 0.99213(9) & 0.0208(3) \\ \mathrm{C} 3 & 0.23747(12) & 0.5973(2) & 0.91781(9) & 0.0217(3) \\ \mathrm{C} 4 & 0.13755(12) & 0.63391(19) & 0.91951(9) & 0.0200(3) \\ \mathrm{C} 5 & 0.12687(11) & 0.68863(18) & 0.99396(8) & 0.0154(3) \\ \mathrm{C} 6 & 0.02538(10) & 0.73730(18) & 0.00170(8) & 0.0154(3) \\ \mathrm{C} 7 & 0.92732(11) & 0.71797(19) & 0.93335(9) & 0.0192(3) \\ \mathrm{C} 8 & 0.83324(11) & 0.7707(2) & 0.94140(9) & 0.0217(3) \\ \mathrm{C} 9 & 0.83429(11) & 0.84533(19) & 0.01598(9) & 0.0209(3) \\ \text { C10 } & 0.92885(11) & 0.86398(19) & 0.08593(8) & 0.0176(3) \\ \text { C11 } & 0.02545(10) & 0.80637(18) & 0.07850(8) & 0.0157(3) \\ \text { C12 } & 0.12732(11) & 0.81760(19) & 0.15249(8) & 0.0166(3) \\ \text { C13 } & 0.92210(12) & 0.9441(2) & 0.16473(9) & 0.0240(3)\end{array}$




$\begin{array}{lcccc} & \mathrm{x} / \mathrm{a} & \mathrm{y} / \mathrm{b} & \mathrm{z} / \mathrm{c} & \mathrm{U}(\mathrm{eq}) \\ \mathrm{C} 14 & 0.31676(10) & 0.75799(19) & 0.21557(8) & 0.0150(3) \\ \mathrm{C} 15 & 0.38375(11) & 0.9038(2) & 0.23626(8) & 0.0182(3) \\ \mathrm{C} 16 & 0.47808(11) & 0.9018(2) & 0.31006(9) & 0.0203(3) \\ \mathrm{C} 17 & 0.50156(11) & 0.7527(2) & 0.36117(8) & 0.0192(3) \\ \text { C18 } & 0.43384(11) & 0.59807(19) & 0.34117(8) & 0.0169(3) \\ \text { C19 } & 0.45540(12) & 0.4381(2) & 0.39002(9) & 0.0223(3) \\ \text { C20 } & 0.38625(12) & 0.2948(2) & 0.36578(9) & 0.0248(3) \\ \text { C21 } & 0.29503(12) & 0.3093(2) & 0.29094(9) & 0.0220(3) \\ \text { C22 } & 0.33916(10) & 0.59981(19) & 0.26677(8) & 0.0151(3)\end{array}$

Interatomic distances $(\AA)$ for $\mathrm{C}_{22} \mathrm{H}_{15} \mathrm{~N}_{3} \mathrm{O}(5 \mathrm{a})$.

$\begin{array}{llll}\text { O1-C12 } & 1.2219(16) & \mathrm{N} 1-\mathrm{C} 12 & 1.3855(17) \\ \mathrm{N} 1-\mathrm{C} 1 & 1.3939(16) & \mathrm{N} 1-\mathrm{C} 14 & 1.4426(16) \\ \mathrm{N} 2-\mathrm{C} 1 & 1.3315(17) & \mathrm{N} 2-\mathrm{C} 2 & 1.3385(17) \\ \mathrm{N} 3-\mathrm{C} 21 & 1.3167(18) & \mathrm{N} 3-\mathrm{C} 22 & 1.3660(18) \\ \mathrm{C} 1-\mathrm{C} 5 & 1.4056(18) & \mathrm{C} 2-\mathrm{C} 3 & 1.381(2) \\ \mathrm{C} 3-\mathrm{C} 4 & 1.380(2) & \mathrm{C} 4-\mathrm{C} 5 & 1.3930(19) \\ \mathrm{C} 5-\mathrm{C} 6 & 1.4623(19) & \mathrm{C} 6-\mathrm{C} 7 & 1.4044(18) \\ \mathrm{C} 6-\mathrm{C} 11 & 1.4079(19) & \mathrm{C} 7-\mathrm{C} 8 & 1.377(2) \\ \mathrm{C} 8-\mathrm{C} 9 & 1.383(2) & \mathrm{C} 9-\mathrm{C} 10 & 1.3890(19) \\ \mathrm{C} 10-\mathrm{C} 11 & 1.4147(19) & \mathrm{C} 10-\mathrm{C} 13 & 1.504(2) \\ \mathrm{C} 11-\mathrm{C} 12 & 1.4792(18) & \mathrm{C} 14-\mathrm{C} 15 & 1.357(2) \\ \mathrm{C} 14-\mathrm{C} 22 & 1.4168(19) & \mathrm{C} 15-\mathrm{C} 16 & 1.4162(19) \\ \mathrm{C} 16-\mathrm{C} 17 & 1.362(2) & \mathrm{C} 17-\mathrm{C} 18 & 1.414(2) \\ \mathrm{C} 18-\mathrm{C} 19 & 1.406(2) & \mathrm{C} 18-\mathrm{C} 22 & 1.4244(18)\end{array}$


$\begin{array}{llll}\mathrm{C} 19-\mathrm{C} 20 & 1.361(2) & \mathrm{C} 20-\mathrm{C} 21 & 1.407(2)\end{array}$

Bond angles $\left({ }^{\circ}\right)$ for $\mathrm{C}_{22} \mathrm{H}_{15} \mathrm{~N}_{3} \mathrm{O}(5 \mathrm{a})$.

$\begin{array}{llll}\text { C12-N1-C1 } & 124.42(11) & \text { C12-N1-C14 } & 116.45(10) \\ \text { C1-N1-C14 } & 119.05(11) & \text { C1-N2-C2 } & 116.89(11) \\ \text { C21-N3-C22 } & 117.24(12) & \text { N2-C1-N1 } & 115.71(11) \\ \text { N2-C1-C5 } & 124.83(12) & \text { N1-C1-C5 } & 119.46(12) \\ \text { N2-C2-C3 } & 123.69(13) & \text { C4-C3-C2 } & 118.19(13) \\ \text { C3-C4-C5 } & 120.56(13) & \text { C4-C5-C1 } & 115.80(12) \\ \text { C4-C5-C6 } & 124.81(12) & \text { C1-C5-C6 } & 119.37(12) \\ \text { C7-C6-C11 } & 119.15(12) & \text { C7-C6-C5 } & 121.09(12) \\ \text { C11-C6-C5 } & 119.76(11) & \text { C8-C7-C6 } & 119.93(13) \\ \text { C7-C8-C9 } & 120.64(13) & \text { C8-C9-C10 } & 121.65(13) \\ \text { C9-C10-C11 } & 117.89(13) & \text { C9-C10-C13 } & 117.96(13) \\ \text { C11-C10-C13 } & 124.15(12) & \text { C6-C11-C10 } & 120.67(12) \\ \text { C6-C11-C12 } & 119.78(12) & \text { C10-C11-C12 } & 119.55(12) \\ \text { O1-C12-N1 } & 118.81(12) & \text { O1-C12-C11 } & 124.14(12) \\ \text { N1-C12-C11 } & 117.05(11) & \text { C15-C14-C22 } & 121.14(12) \\ \text { C15-C14-N1 } & 120.59(12) & \text { C22-C14-N1 } & 118.26(12) \\ \text { C14-C15-C16 } & 120.64(13) & \text { C17-C16-C15 } & 119.79(13) \\ \text { C16-C17-C18 } & 120.99(12) & \text { C19-C18-C17 } & 123.65(12) \\ \text { C19-C18-C22 } & 117.10(13) & \text { C17-C18-C22 } & 119.24(12) \\ \text { C20-C19-C18 } & 119.83(13) & \text { C19-C20-C21 } & 118.84(14) \\ \text { N3-C21-C20 } & 124.22(14) & \text { N3-C22-C14 } & 119.03(12) \\ \text { N3-C22-C18 } & 122.76(12) & \text { C14-C22-C18 } & 118.20(12) \\ & & & \\ & & & \\ \text { N } 13)\end{array}$


Anisotropic atomic displacement parameters $\left(\AA^{2}\right)$ for $\mathrm{C}_{22} \mathrm{H}_{15} \mathrm{~N}_{3} \mathrm{O}(5 \mathrm{a})$.

The anisotropic atomic displacement factor exponent takes the form: $-2 \pi^{2}[$ $\left.\mathbf{h}^{2} \mathrm{a}^{* 2} \mathrm{U}_{11}+\ldots+2 \mathrm{~h} \mathrm{k} \mathbf{a}^{*} \mathbf{b}^{*} \mathrm{U}_{12}\right]$

$\begin{array}{llllll}\mathrm{U}_{11} & \mathrm{U}_{22} & \mathrm{U}_{33} & \mathrm{U}_{23} & \mathrm{U}_{13} & \mathrm{U}_{12}\end{array}$

$\begin{array}{lllllll}\mathrm{O} 1 & 0.0199(5) & 0.0430(7) & 0.0154(5) & -0.0053(5) & 0.0044(4) & 0.0042(5)\end{array}$

$\begin{array}{lllllll}\mathrm{N} 1 & 0.0130(5) & 0.0176(6) & 0.0110(5) & 0.0010(4) & 0.0015(4) & 0.0010(4)\end{array}$

$\begin{array}{lllllll}\mathrm{N} 2 & 0.0179(6) & 0.0206(6) & 0.0156(6) & -0.0006(5) & 0.0049(5) & 0.0017(5)\end{array}$

$\begin{array}{lllllll}\mathrm{N} 3 & 0.0182(6) & 0.0191(6) & 0.0168(6) & -0.0019(5) & 0.0062(5) & -0.0006(5)\end{array}$

$\begin{array}{lllllll}\mathrm{C} 1 & 0.0181(6) & 0.0121(6) & 0.0135(6) & 0.0016(5) & 0.0052(5) & 0.0005(5)\end{array}$

$\begin{array}{lllllll}\mathrm{C} 2 & 0.0211(7) & 0.0236(7) & 0.0185(7) & -0.0005(6) & 0.0083(6) & 0.0036(6)\end{array}$

$\begin{array}{lllllll}\mathrm{C} 3 & 0.0289(8) & 0.0218(7) & 0.0140(7) & -0.0013(5) & 0.0075(6) & 0.0048(6)\end{array}$

$\begin{array}{lllllll}\mathrm{C} 4 & 0.0238(7) & 0.0173(7) & 0.0133(6) & -0.0003(5) & 0.0007(5) & 0.0016(6)\end{array}$

$\begin{array}{lllllll}\mathrm{C} 5 & 0.0181(6) & 0.0124(6) & 0.0130(6) & 0.0018(5) & 0.0027(5) & 0.0001(5)\end{array}$

$\begin{array}{lllllll}\mathrm{C} 6 & 0.0168(6) & 0.0107(6) & 0.0155(6) & 0.0030(5) & 0.0022(5) & 0.0006(5)\end{array}$

$\begin{array}{lllllll}\mathrm{C} 7 & 0.0216(7) & 0.0151(7) & 0.0158(6) & 0.0005(5) & 0.0013(5) & -0.0017(6)\end{array}$

$\begin{array}{lllllll}\mathrm{C} 8 & 0.0162(6) & 0.0190(7) & 0.0216(7) & 0.0027(6) & -0.0023(5) & -0.0016(6)\end{array}$

$\begin{array}{lllllll}\text { C9 } & 0.0150(6) & 0.0188(7) & 0.0271(8) & 0.0047(6) & 0.0058(6) & 0.0007(6)\end{array}$

$\begin{array}{lllllll}\mathrm{C} 10 & 0.0167(6) & 0.0150(6) & 0.0205(7) & 0.0045(5) & 0.0061(5) & 0.0003(5)\end{array}$

$\begin{array}{lllllll}\mathrm{C} 11 & 0.0154(6) & 0.0141(6) & 0.0148(6) & 0.0034(5) & 0.0024(5) & -0.0006(5)\end{array}$

$\begin{array}{lllllll}\mathrm{C} 12 & 0.0165(6) & 0.0175(7) & 0.0151(6) & 0.0010(5) & 0.0049(5) & 0.0001(5)\end{array}$

$\begin{array}{lllllll}\mathrm{C} 13 & 0.0187(7) & 0.0307(8) & 0.0229(7) & 0.0034(6) & 0.0083(6) & 0.0044(6)\end{array}$

$\begin{array}{lllllll}\mathrm{C} 14 & 0.0130(6) & 0.0212(7) & 0.0103(6) & -0.0017(5) & 0.0039(5) & 0.0019(5)\end{array}$

$\begin{array}{lllllll}\mathrm{C} 15 & 0.0192(7) & 0.0202(7) & 0.0155(6) & -0.0001(5) & 0.0069(5) & 0.0001(6)\end{array}$

$\begin{array}{lllllll}\mathrm{C} 16 & 0.0166(7) & 0.0247(7) & 0.0203(7) & -0.0063(6) & 0.0076(6) & -0.0043(6)\end{array}$

$\begin{array}{lllllll}\mathrm{C} 17 & 0.0134(6) & 0.0283(8) & 0.0133(6) & -0.0047(6) & 0.0019(5) & 0.0013(6)\end{array}$ 


$\begin{array}{ccccccc} & \mathrm{U}_{11} & \mathrm{U}_{22} & \mathrm{U}_{33} & \mathrm{U}_{23} & \mathrm{U}_{13} & \mathrm{U}_{12} \\ \mathrm{C} 18 & 0.0157(6) & 0.0227(7) & 0.0125(6) & -0.0019(5) & 0.0054(5) & 0.0041(6) \\ \mathrm{C} 19 & 0.0209(7) & 0.0292(8) & 0.0149(7) & 0.0001(6) & 0.0045(5) & 0.0083(6) \\ \mathrm{C} 20 & 0.0319(8) & 0.0212(7) & 0.0224(7) & 0.0057(6) & 0.0115(6) & 0.0070(6) \\ \mathrm{C} 21 & 0.0248(7) & 0.0196(7) & 0.0236(7) & -0.0006(6) & 0.0115(6) & 0.0003(6) \\ \mathrm{C} 22 & 0.0150(6) & 0.0187(7) & 0.0120(6) & -0.0017(5) & 0.0056(5) & 0.0018(5)\end{array}$

Hydrogen atom coordinates and isotropic atomic displacement parameters $\left(\AA^{2}\right)$ for $\mathrm{C}_{22} \mathrm{H}_{15} \mathrm{~N}_{3} \mathrm{O}(5 \mathrm{a})$.

$\begin{array}{ccccc} & \mathrm{x} / \mathrm{a} & \mathrm{y} / \mathrm{b} & \mathrm{z} / \mathrm{c} & \mathrm{U}(\mathrm{eq}) \\ \mathrm{H} 2 & 0.3943 & 0.5848 & -0.0089 & 0.025 \\ \text { H3 } & 0.2454 & 0.5629 & -0.1331 & 0.026 \\ \text { H4 } & 0.0755 & 0.6217 & -0.1305 & 0.024 \\ \text { H7 } & -0.0741 & 0.6685 & -0.1184 & 0.023 \\ \text { H8 } & -0.2330 & 0.7558 & -0.1047 & 0.026 \\ \text { H9 } & -0.2313 & 0.8848 & 0.0194 & 0.025 \\ \text { H13A } & -0.1504 & 0.9916 & 0.1524 & 0.036 \\ \text { H13B } & -0.0257 & 1.0434 & 0.1850 & 0.036 \\ \text { H13C } & -0.0620 & 0.8495 & 0.2081 & 0.036 \\ \text { H15 } & 0.3672 & 1.0083 & 0.2009 & 0.022 \\ \text { H16 } & 0.5248 & 1.0041 & 0.3239 & 0.024 \\ \text { H17 } & 0.5644 & 0.7526 & 0.4110 & 0.023 \\ \text { H19 } & 0.5181 & 0.4301 & 0.4398 & 0.027 \\ \text { H20 } & 0.3993 & 0.1869 & 0.3988 & 0.03 \\ \text { H21 } & 0.2479 & 0.2077 & 0.2745 & 0.026\end{array}$




\section{Characterization of (Hetero)Aryl Benzamides (1)}<smiles>Cc1ccc(C(=O)Nc2cccc3cccnc23)c(C)c1</smiles>

2,4-dimethyl- $N$-(quinolin-8-yl)benzamide (1q). The title compound was synthesized following General Procedure B. ${ }^{1} \mathrm{H}$ NMR (400 MHz, $\left.\mathrm{CDCl}_{3}\right): \delta=10.22$ (s, 1H), 8.94 (dd, $J=7.5,1.5 \mathrm{~Hz}, 1 \mathrm{H}), 8.77(\mathrm{dd}, J=4.2,1.7 \mathrm{~Hz}, 1 \mathrm{H}), 8.17(\mathrm{dd}, J=8.3,1.7 \mathrm{~Hz}, 1 \mathrm{H}), 7.65-$ $7.57(\mathrm{~m}, 2 \mathrm{H}), 7.54(\mathrm{dd}, J=8.3,1.5 \mathrm{~Hz}, 1 \mathrm{H}), 7.45(\mathrm{dd}, J=8.3,4.2 \mathrm{~Hz}, 1 \mathrm{H}), 7.18-7.09$ $(\mathrm{m}, 2 \mathrm{H}), 2.59(\mathrm{~s}, 3 \mathrm{H}), 2.39(\mathrm{~s}, 3 \mathrm{H}) .{ }^{13} \mathrm{C}\left\{{ }^{1} \mathrm{H}\right\} \mathrm{NMR}\left(100 \mathrm{MHz}, \mathrm{CDCl}_{3}\right): \delta=168.22$, $148.25,140.55,138.65,136.88,136.35,134.91$, 133.76, 132.25, 128.03, 127.46, 127.45, $126.69,121.66,121.66,116.42,21.37,20.34$. The spectral data are consistent with those reported in the literature. ${ }^{70}$<smiles>COc1ccc(C(=O)Nc2cccc3cccnc23)c(C)c1</smiles>

4-methoxy-2-methyl- $N$-(quinolin-8-yl)benzamide (1r). The title compound was synthesized following General Procedure B. ${ }^{1} \mathrm{H}$ NMR $\left(400 \mathrm{MHz}, \mathrm{CDCl}_{3}\right): \delta=10.21(\mathrm{~s}$, $1 \mathrm{H}), 8.92(\mathrm{dd}, J=7.5,1.5 \mathrm{~Hz}, 1 \mathrm{H}), 8.79(\mathrm{dd}, J=4.2,1.7 \mathrm{~Hz}, 1 \mathrm{H}), 8.18(\mathrm{dd}, J=8.3,1.7$ $\mathrm{Hz}, 1 \mathrm{H}), 7.72-7.67(\mathrm{~m}, 1 \mathrm{H}), 7.59(\mathrm{dd}, J=8.3,7.5 \mathrm{~Hz}, 1 \mathrm{H}), 7.54(\mathrm{dd}, J=8.3,1.5 \mathrm{~Hz}$, $1 \mathrm{H}), 7.46(\mathrm{dd}, J=8.2,4.2 \mathrm{~Hz}, 1 \mathrm{H}), 6.88-6.81(\mathrm{~m}, 2 \mathrm{H}), 3.87(\mathrm{~s}, 3 \mathrm{H}), 2.62(\mathrm{~s}, 3 \mathrm{H})$. ${ }^{13} \mathrm{C}\left\{{ }^{1} \mathrm{H}\right\} \mathrm{NMR}\left(100 \mathrm{MHz}, \mathrm{CDCl}_{3}\right): \delta=167.82,161.10,148.26,139.47,138.69,136.41$, $135.01,129.33$, 129.04, 128.07, 127.50, 121.69, 121.57, 117.01, 116.38, 111.12, 55.39, 20.91. The spectral data are consistent with those reported in the literature. ${ }^{70}$ 
<smiles>COc1cc(Cl)ccc1C(=O)Nc1cccc2cccnc12</smiles>

4-chloro-2-methyl- $N$-(quinolin-8-yl)benzamide (1s). The title compound was synthesized following General Procedure B. ${ }^{1} \mathrm{H}$ NMR $\left(400 \mathrm{MHz}, \mathrm{CDCl}_{3}\right): \delta=10.19(\mathrm{~s}$, 1H), $8.91(\mathrm{dd}, J=7.3,1.7 \mathrm{~Hz}, 1 \mathrm{H}), 8.78(\mathrm{dd}, J=4.2,1.7 \mathrm{~Hz}, 1 \mathrm{H}), 8.19$ (dd, $J=8.3,1.7$ $\mathrm{Hz}, 1 \mathrm{H}), 7.66-7.54(\mathrm{~m}, 3 \mathrm{H}), 7.47$ (dd, $J=8.3,4.2 \mathrm{~Hz}, 1 \mathrm{H}), 7.33-7.28$ (m, 2H), 2.59 (s, $3 \mathrm{H}) .{ }^{13} \mathrm{C}\left\{{ }^{1} \mathrm{H}\right\} \mathrm{NMR}\left(100 \mathrm{MHz}, \mathrm{CDCl}_{3}\right): \delta=167.10,148.38,138.90,138.57,136.44$, $136.15,134.99$, 134.55, 131.33, 128.69, 128.03, 127.42, 126.21, 122.03, 121.78, 116.60, 20.21. The spectral data are consistent with those reported in the literature. ${ }^{70}$<smiles>Cc1cccc(C(=O)Nc2cccc3cccnc23)c1C</smiles>

2,3-dimethyl- $N$-(quinolin-8-yl)benzamide (1u). The title compound was synthesized following General Procedure B. ${ }^{1} \mathrm{H}$ NMR $\left(400 \mathrm{MHz}, \mathrm{CDCl}_{3}\right): \delta=10.13(\mathrm{~s}, 1 \mathrm{H}), 8.96$ (dd, $J=7.5,1.5 \mathrm{~Hz}, 1 \mathrm{H}), 8.76(\mathrm{dd}, J=4.2,1.7 \mathrm{~Hz}, 1 \mathrm{H}), 8.18(\mathrm{dd}, J=8.3,1.7 \mathrm{~Hz}, 1 \mathrm{H}), 7.65-$ $7.53(\mathrm{~m}, 2 \mathrm{H}), 7.51-7.42(\mathrm{~m}, 2 \mathrm{H}), 7.29(\mathrm{~d}, J=7.0 \mathrm{~Hz}, 1 \mathrm{H}), 7.22(\mathrm{t}, J=7.5 \mathrm{~Hz}, 1 \mathrm{H}), 2.46$ (s, 3H), $2.36(\mathrm{~s}, 3 \mathrm{H}) .{ }^{13} \mathrm{C}\left\{{ }^{1} \mathrm{H}\right\}$ NMR $\left(100 \mathrm{MHz}, \mathrm{CDCl}_{3}\right): \delta=168.97,148.22,138.51$, $138.10,137.57,136.28,134.74,134.56,131.55,127.95,127.34,125.70,124.71,121.77$, $121.63,116.46,20.36,16.50$. The spectral data are consistent with those reported in the literature. $^{71}$ 
<smiles>COc1cccc(C(=O)Nc2cccc3cccnc23)c1OC</smiles>

3-methoxy-2-methyl- $N$-(quinolin-8-yl)benzamide (1t). The title compound was synthesized following General Procedure B. ${ }^{1} \mathrm{H}$ NMR $\left(400 \mathrm{MHz}, \mathrm{CDCl}_{3}\right): \delta=10.15(\mathrm{~s}$, $1 \mathrm{H}), 8.95(\mathrm{dd}, J=7.6,1.5 \mathrm{~Hz}, 1 \mathrm{H}), 8.76(\mathrm{dd}, J=4.2,1.7 \mathrm{~Hz}, 1 \mathrm{H}), 8.18(\mathrm{dd}, J=8.3,1.7$ $\mathrm{Hz}, 1 \mathrm{H}), 7.65-7.51(\mathrm{~m}, 2 \mathrm{H}), 7.45(\mathrm{dd}, J=8.3,4.2 \mathrm{~Hz}, 1 \mathrm{H}), 7.34-7.22(\mathrm{~m}, 2 \mathrm{H}), 6.98$ $(\mathrm{dd}, J=7.9,1.5 \mathrm{~Hz}, 1 \mathrm{H}), 3.89$ (s, 3H), $2.43(\mathrm{~s}, 3 \mathrm{H}) .{ }^{13} \mathrm{C}\left\{{ }^{1} \mathrm{H}\right\}$ NMR $\left(100 \mathrm{MHz}, \mathrm{CDCl}_{3}\right): \delta$ $=168.28,158.23,148.29,138.58,138.43,136.35,134.73,128.01,127.41,126.84$, $125.17,121.84,121.70,119.12,116.53,111.81,55.75,12.88$. The spectral data are consistent with those reported in the literature. ${ }^{71}$<smiles>Cc1c(Cl)cccc1C(=O)Nc1cccc2cccnc12</smiles>

3-chloro-2-methyl- $N$-(quinolin-8-yl)benzamide (1v). The title compound was synthesized following General Procedure B as a white solid in 58\% yield (293 mg, 0.98 mmol), mp $=161-163{ }^{\circ} \mathrm{C}$. ${ }^{1} \mathrm{H}$ NMR $\left(400 \mathrm{MHz}, \mathrm{CDCl}_{3}\right): \delta=10.14$ (s, 1H), 8.93 (dd, $J=$ 7.2, $1.8 \mathrm{~Hz}, 1 \mathrm{H}), 8.77$ (dd, $J=4.2,1.7 \mathrm{~Hz}, 1 \mathrm{H}), 8.19$ (dd, $J=8.3,1.7 \mathrm{~Hz}, 1 \mathrm{H}), 7.66-$ $7.42(\mathrm{~m}, 5 \mathrm{H}), 7.26(\mathrm{t}, J=7.8 \mathrm{~Hz}, 1 \mathrm{H}), 2.59(\mathrm{~s}, 3 \mathrm{H}) .{ }^{13} \mathrm{C}\left\{{ }^{1} \mathrm{H}\right\} \mathrm{NMR}\left(100 \mathrm{MHz}, \mathrm{CDCl}_{3}\right): \delta$ $=167.45,148.42,139.12,138.56,136.46,136.09,134.47,134.43,131.03,128.05$, 127.42, 127.07, 125.53, 122.19, 121.82, 116.74, 17.29. FTIR (ATR, cm ${ }^{-1}$ ): 3359, 3061, 1674, 1527, 1486, 1427, 1382, 1329, 788, 663. HRMS (ESI-MS) m/z calcd for $\mathrm{C}_{17} \mathrm{H}_{14} \mathrm{ClN}_{2} \mathrm{O}[\mathrm{M}+\mathrm{H}]^{+} 297.0794$ found 297.0793. 
<smiles>Cc1cnccc1C(=O)Nc1cccc2cccnc12</smiles>

3-methyl- $N$-(quinolin-8-yl)pyridine-4-carboxamide (1x). The title compound was synthesized following General Procedure B. ${ }^{1} \mathrm{H}$ NMR (400 MHz, $\left.\mathrm{CDCl}_{3}\right): \delta=10.27(\mathrm{~s}$, $1 \mathrm{H}), 8.91(\mathrm{dd}, J=6.3,2.7 \mathrm{~Hz}, 1 \mathrm{H}), 8.80(\mathrm{dd}, J=4.2,1.7 \mathrm{~Hz}, 1 \mathrm{H}), 8.62(\mathrm{~d}, J=1.0 \mathrm{~Hz}$, 2H), $8.20(\mathrm{dd}, J=8.3,1.7 \mathrm{~Hz}, 1 \mathrm{H}), 7.66-7.57(\mathrm{~m}, 2 \mathrm{H}), 7.54(\mathrm{~d}, J=5.0 \mathrm{~Hz}, 1 \mathrm{H}), 7.48$ $(\mathrm{dd}, J=8.3,4.2 \mathrm{~Hz}, 1 \mathrm{H}), 2.59(\mathrm{~s}, 3 \mathrm{H}) .{ }^{13} \mathrm{C}\left\{{ }^{1} \mathrm{H}\right\} \mathrm{NMR}\left(100 \mathrm{MHz}, \mathrm{CDCl}_{3}\right): \delta=165.70$, 152.53, 148.52, 148.06, 143.03, 138.52, 136.49, 134.11, 130.84, 128.02, 127.36, 122.47, $121.90,120.73,116.86,17.05$. The spectral data are consistent with those reported in the literature. $^{70}$

\section{Characterization of ortho-Fluoro Heteroaromatic Carboxylic Acids (4)}<smiles>Cc1ccc(C(=O)O)c(F)n1</smiles>

2-fluoro-6-methylpyridine-3-carboxylic acid (4b). The title compound was synthesized following Procedure A for the Synthesis of Fluorinated Carboxylic Acids as a white solid in $91 \%$ yield, mp $=182-184{ }^{\circ} \mathrm{C} .{ }^{1} \mathrm{H}$ NMR $\left(400 \mathrm{MHz}, \mathrm{DMSO}-d_{6}\right): \delta=13.40(\mathrm{~s}, 1 \mathrm{H})$, $8.25(\mathrm{dd}, J=9.9,7.7 \mathrm{~Hz}, 1 \mathrm{H}), 7.30(\mathrm{dd}, J=7.8,1.8 \mathrm{~Hz}, 1 \mathrm{H}), 2.47(\mathrm{~s}, 3 \mathrm{H}) .{ }^{13} \mathrm{C}\left\{{ }^{1} \mathrm{H}\right\} \mathrm{NMR}$ $\left(100 \mathrm{MHz}, \mathrm{DMSO}-d_{6}\right): \delta=164.08(\mathrm{~d}, J=7.7 \mathrm{~Hz}), 161.91(\mathrm{~d}, J=15.1 \mathrm{~Hz}), 160.26(\mathrm{~d}, J=$ $245.6 \mathrm{~Hz}), 143.58$ (d, $J=1.8 \mathrm{~Hz}), 121.43$ (d, $J=4.6 \mathrm{~Hz}), 110.92$ (d, $J=24.8 \mathrm{~Hz}), 23.56$. ${ }^{19}$ F NMR (376 MHz, DMSO- $\left.d_{6}\right): \delta=-64.43(\mathrm{~d}, J=10.0 \mathrm{~Hz})$. FTIR (ATR, $\left.\mathrm{cm}^{-1}\right): 2918$, 2592, 1725, 1616, 1478, 1243, 1215, 1083, 779, 726. HRMS (ESI-MS) m/z calcd for $\mathrm{C}_{7} \mathrm{H}_{7} \mathrm{FNO}_{2}[\mathrm{M}+\mathrm{H}]^{+} 156.0460$ found 156.0458 . 
<smiles>COc1ccc(C(=O)O)c(F)n1</smiles>

2-fluoro-6-methoxypyridine-3-carboxylic acid (4c). The title compound was synthesized following Procedure A for the Synthesis of Fluorinated Carboxylic Acids as a white solid in $33 \%$ yield, $\mathrm{mp}=210-212{ }^{\circ} \mathrm{C} .{ }^{1} \mathrm{H}$ NMR $\left(400 \mathrm{MHz}, \mathrm{DMSO}-d_{6}\right): \delta=$ $13.18(\mathrm{~s}, 1 \mathrm{H}), 8.24(\mathrm{dd}, J=9.8,8.4 \mathrm{~Hz}, 1 \mathrm{H}), 6.81(\mathrm{dd}, J=8.4,1.0 \mathrm{~Hz}, 1 \mathrm{H}), 3.88(\mathrm{~s}, 3 \mathrm{H})$. ${ }^{13} \mathrm{C}\left\{{ }^{1} \mathrm{H}\right\}$ NMR $\left(100 \mathrm{MHz}, \mathrm{DMSO}-d_{6}\right): \delta=165.18(\mathrm{~d}, J=14.5 \mathrm{~Hz}), 163.87(\mathrm{~d}, J=7.6$ Hz), 160.67 (d, $J=251.0 \mathrm{~Hz}), 145.45$ (d, $J=1.9 \mathrm{~Hz}), 107.79$ (d, $J=5.1 \mathrm{~Hz}), 105.27$ (d, $J$ $=22.4 \mathrm{~Hz}), 54.60 .{ }^{19} \mathrm{~F}$ NMR $\left(376 \mathrm{MHz}, \mathrm{DMSO}-d_{6}\right): \delta-63.13(\mathrm{~d}, J=9.8 \mathrm{~Hz})$. FTIR $\left(\right.$ ATR, $\left.\mathrm{cm}^{-1}\right):$ 2962, 2571, 1670, 1610, 1499, 1412, 1399, 1303, 1144, 1017, 930, 847, 789. HRMS (ESI-MS) m/z calcd forC ${ }_{7} \mathrm{H}_{7} \mathrm{FNO}_{3}[\mathrm{M}+\mathrm{H}]^{+} 172.0409$ found 172.0406 .<smiles>O=C(O)c1cc2ccccc2nc1F</smiles>

2-fluoroquinoline-3-carboxylic acid (4h). The title compound was synthesized following Procedure A at $50{ }^{\circ} \mathrm{C}$ for Synthesis of Fluorinated Carboxylic Acids as a white solid in $73 \%$ yield, $\mathrm{mp}=258-260{ }^{\circ} \mathrm{C} .{ }^{1} \mathrm{H}$ NMR (400 MHz, DMSO- $\left.d_{6}\right): \delta=13.65(\mathrm{~s}$, $1 \mathrm{H}), 9.12(\mathrm{~d}, J=9.8 \mathrm{~Hz}, 1 \mathrm{H}), 8.23(\mathrm{~d}, J=8.1 \mathrm{~Hz}, 1 \mathrm{H}), 7.92(\mathrm{dd}, J=4.5,1.1 \mathrm{~Hz}, 2 \mathrm{H})$, $7.70(\mathrm{dt}, J=8.2,3.9 \mathrm{~Hz}, 1 \mathrm{H}) .{ }^{13} \mathrm{C}\left\{{ }^{1} \mathrm{H}\right\} \mathrm{NMR}\left(100 \mathrm{MHz}, \mathrm{DMSO}-d_{6}\right): \delta=163.91(\mathrm{~d}, J=$ $7.5 \mathrm{~Hz}), 157.75$ (d, $J=247.6 \mathrm{~Hz}), 146.19$ (d, $J=17.5 \mathrm{~Hz}), 145.72(\mathrm{~d}, J=4.0 \mathrm{~Hz})$, 133.03, 129.30, 127.10 (d, $J=1.5 \mathrm{~Hz}), 127.00(\mathrm{~d}, J=2.3 \mathrm{~Hz}), 126.14$ (d, $J=2.3 \mathrm{~Hz})$, $114.43(\mathrm{~d}, J=30.8 \mathrm{~Hz}) .{ }^{19} \mathrm{~F}$ NMR $\left(376 \mathrm{MHz}, \mathrm{DMSO}-d_{6}\right): \delta=-56.49(\mathrm{~d}, J=9.5 \mathrm{~Hz})$. FTIR (ATR, $\mathrm{cm}^{-1}$ ): 2971, 2585, 1695, 1620, 1601, 1472, 1417, 1264, 1078, 915, 788, 750. HRMS (ESI-MS) m/z calcd for $\mathrm{C}_{10} \mathrm{H}_{7} \mathrm{FNO}_{2}[\mathrm{M}+\mathrm{H}]^{+} 192.0460$ found 192.0458 . 


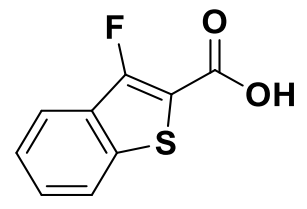

3-fluoro-1-benzothiophene-2-carboxylic acid (4i). The title compound was synthesized following Procedure B for Synthesis of Fluorinated Carboxylic Acids as a white solid in $83 \%, \mathrm{mp}=250-252{ }^{\circ} \mathrm{C} .{ }^{1} \mathrm{H}$ NMR $\left(400 \mathrm{MHz}, \mathrm{DMSO}-d_{6}\right): \delta=13.71(\mathrm{~s}, 1 \mathrm{H}), 8.03(\mathrm{~d}, J$ $=8.2 \mathrm{~Hz}, 1 \mathrm{H}), 7.88(\mathrm{dd}, J=8.1,0.6 \mathrm{~Hz}, 1 \mathrm{H}), 7.60(\mathrm{td}, J=8.3,7.1,1.3 \mathrm{~Hz}, 1 \mathrm{H}), 7.52(\mathrm{td}$, $J=8.1,7.1,1.0 \mathrm{~Hz}, 1 \mathrm{H}) .{ }^{13} \mathrm{C}\left\{{ }^{1} \mathrm{H}\right\} \mathrm{NMR}\left(100 \mathrm{MHz}, \mathrm{DMSO}-d_{6}\right): \delta=161.74(\mathrm{~d}, J=3.2$ $\mathrm{Hz}), 152.66$ (d, $J=279.2 \mathrm{~Hz}), 136.14$ (d, $J=8.6 \mathrm{~Hz}), 128.64,128.41$ (d, $J=22.5 \mathrm{~Hz})$, 125.63, $123.70(\mathrm{~d}, J=1.4 \mathrm{~Hz}), 121.46(\mathrm{~d}, J=1.8 \mathrm{~Hz}), 112.95(\mathrm{~d}, J=8.9 \mathrm{~Hz}) .{ }^{19} \mathrm{~F}$ NMR (376 MHz, DMSO- $d_{6}$ ): $\delta=-121.02$. FTIR (ATR, $\mathrm{cm}^{-1}$ ): 2805, 2569, 1663, 1599, 1574, 1537, 1446, 1369, 1289, 1043, 900, 755, 729. HRMS (ESI-MS) m/z calcd for $\mathrm{C}_{9} \mathrm{H}_{6} \mathrm{FO}_{2} \mathrm{~S}$ $[\mathrm{M}+\mathrm{H}]^{+} 197.0072$ found 197.0071 .

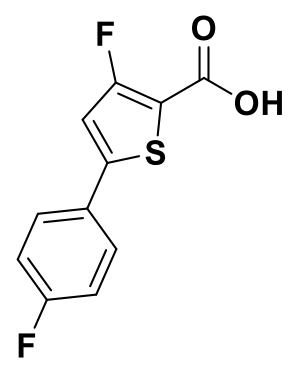

3-fluoro-5-(4-fluorophenyl)thiophene-2-carboxylic acid (4j). The title compound was synthesized following Procedure B for Synthesis of Fluorinated Carboxylic Acids as a white solid in $85 \%$ yield, $\mathrm{mp}=248-250{ }^{\circ} \mathrm{C} .{ }^{1} \mathrm{H}$ NMR $\left(400 \mathrm{MHz}, \mathrm{DMSO}-d_{6}\right): \delta=13.34$ (s, 1H), $7.82-7.73(\mathrm{~m}, 2 \mathrm{H}), 7.55(\mathrm{~s}, 1 \mathrm{H}), 7.34-7.23(\mathrm{~m}, 2 \mathrm{H}) .{ }^{13} \mathrm{C}\left\{{ }^{1} \mathrm{H}\right\} \mathrm{NMR}(100 \mathrm{MHz}$, DMSO- $\left.d_{6}\right): \delta=162.81(\mathrm{~d}, J=247.9 \mathrm{~Hz}), 160.98(\mathrm{~d}, J=3.1 \mathrm{~Hz}), 158.78(\mathrm{~d}, J=273.3$ $\mathrm{Hz}), 145.25$ (d, $J=9.8 \mathrm{~Hz}), 128.69$ (d, $J=3.2 \mathrm{~Hz}), 127.73$ (d, $J=8.6 \mathrm{~Hz}), 116.30$ (d, $J=$ $22.1 \mathrm{~Hz}), 115.14(\mathrm{~d}, J=25.9 \mathrm{~Hz}), 112.00$ (d, $J=9.9 \mathrm{~Hz}) .{ }^{19} \mathrm{~F}$ NMR $(376 \mathrm{MHz}, \mathrm{DMSO}-$ $\left.d_{6}\right): \delta=-111.40(\mathrm{tt}, J=8.7,5.2 \mathrm{~Hz}),-112.89$. FTIR (ATR, $\left.\mathrm{cm}^{-1}\right): 3096,2550,1650,1598$, 1560, 1459, 1442, 1295, 1227, 1161, 1041, 933, 823, 809, 762, 716. HRMS (ESI-MS) $\mathrm{m} / \mathrm{z}$ calcd for $\mathrm{C}_{11} \mathrm{H}_{7} \mathrm{~F}_{2} \mathrm{O}_{2} \mathrm{~S}[\mathrm{M}+\mathrm{H}]^{+} 241.0134$ found 241.0143 . 
<smiles>Cc1cnc(F)c(C(=O)O)c1</smiles>

2-fluoro-5-methylpyridine-3-carboxylic acid (4f). The title compound was synthesized following Procedure $\mathrm{C}$ for Synthesis of Fluorinated Carboxylic Acids as a white solid in $79 \%$ yield, $\mathrm{mp}=178-180{ }^{\circ} \mathrm{C} .{ }^{1} \mathrm{H}$ NMR $\left(400 \mathrm{MHz}, \mathrm{DMSO}-d_{6}\right): \delta=13.47(\mathrm{~s}, 1 \mathrm{H}), 8.19-$ $8.08(\mathrm{~m}, 2 \mathrm{H}), 2.28(\mathrm{~s}, 3 \mathrm{H}) .{ }^{13} \mathrm{C}\left\{{ }^{1} \mathrm{H}\right\} \mathrm{NMR}\left(100 \mathrm{MHz}, \mathrm{DMSO}-d_{6}\right): \delta=164.22(\mathrm{~d}, J=7.6$ Hz), 159.49 (d, $J=243.5 \mathrm{~Hz}), 150.88$ (d, $J=15.2 \mathrm{~Hz}), 143.51$ (d, $J=1.6 \mathrm{~Hz}), 131.67$ (d, $J=5.1 \mathrm{~Hz}), 113.42(\mathrm{~d}, J=25.7 \mathrm{~Hz}), 16.70 .{ }^{19} \mathrm{~F}$ NMR $\left(376 \mathrm{MHz}, \mathrm{DMSO}-d_{6}\right): \delta=-69.68$ $(\mathrm{d}, J=9.0 \mathrm{~Hz})$. FTIR $\left(\right.$ ATR, $\left.\mathrm{cm}^{-1}\right): 2852,2610,1681,1605,1463,1312,1209,1113,906$, 789, 748. HRMS (ESI-MS) m/z calcd for $\mathrm{C}_{7} \mathrm{H}_{7} \mathrm{FNO}_{2}[\mathrm{M}+\mathrm{H}]^{+} 156.0460$ found 156.0458 .

\section{Characterization of Oxidative Decarboxylative Annulation Products (5)}<smiles>Cc1cccc2c1c(=O)n(-c1cccc3cccnc13)c1ncccc21</smiles>

7-methyl-5-(quinolin-8-yl)-5H,6H-benzo[c]1,8-naphthyridin-6-one (5a). The title compound was synthesized following the General Procedure A as an off-white solid in $80 \%$ yield $(53.9 \mathrm{mg}, 0.16 \mathrm{mmol}), \mathrm{mp}=271-273{ }^{\circ} \mathrm{C} .{ }^{1} \mathrm{H} \mathrm{NMR}\left(400 \mathrm{MHz}, \mathrm{CDCl}_{3}\right): \delta=$ $8.75(\mathrm{dd}, J=4.1,1.5 \mathrm{~Hz}, 1 \mathrm{H}), 8.54(\mathrm{dd}, J=8.0,1.6 \mathrm{~Hz}, 1 \mathrm{H}), 8.27-8.17(\mathrm{~m}, 3 \mathrm{H}), 7.98$ $(\mathrm{dt}, J=7.6,3.8 \mathrm{~Hz}, 1 \mathrm{H}), 7.79-7.73(\mathrm{~m}, 2 \mathrm{H}), 7.66(\mathrm{t}, J=7.8 \mathrm{~Hz}, 1 \mathrm{H}), 7.43(\mathrm{~d}, J=7.5$ $\mathrm{Hz}, 1 \mathrm{H}), 7.38$ (dd, $J=8.3,4.2 \mathrm{~Hz}, 1 \mathrm{H}), 7.12$ (dd, $J=7.9,4.7 \mathrm{~Hz}, 1 \mathrm{H}), 2.92$ (s, 3H). ${ }^{13} \mathrm{C}\left\{{ }^{1} \mathrm{H}\right\} \mathrm{NMR}\left(100 \mathrm{MHz}, \mathrm{CDCl}_{3}\right): \delta=163.71,150.89,150.36,148.85,144.82,143.58$, $136.59,136.49,134.45,132.58,132.14$, 131.66, 130.39, 129.69, 128.87, 126.72, 124.70, 121.67, 120.29, 118.21, 114.86, 24.50. FTIR (ATR, $\mathrm{cm}^{-1}$ ): 3030, 2972, 1660, 1584, 1499, 
1473, 1394, 1288, 825, 773. HRMS (ESI-MS) m/z calcd for $\mathrm{C}_{22} \mathrm{H}_{16} \mathrm{~N}_{3} \mathrm{O}[\mathrm{M}+\mathrm{H}]^{+}$ 338.1293 found 338.1291 .<smiles>Cc1ccc2c3cccc(C)c3c(=O)n(-c3cccc4cccnc34)c2n1</smiles>

3,7-dimethyl-5-(quinolin-8-yl)-5H,6H-benzo[c]1,8-naphthyridin-6-one (5b). The title compound was synthesized following the General Procedure A and a light brown solid in $67 \%$ yield $(47.0 \mathrm{mg}, 0.13 \mathrm{mmol}), \mathrm{mp}=256-258{ }^{\circ} \mathrm{C} .{ }^{1} \mathrm{H} \mathrm{NMR}\left(400 \mathrm{MHz}, \mathrm{CDCl}_{3}\right): \delta=$ $8.75(\mathrm{dd}, J=4.2,1.7 \mathrm{~Hz}, 1 \mathrm{H}), 8.39$ (d, $J=8.0 \mathrm{~Hz}, 1 \mathrm{H}), 8.23(\mathrm{dd}, J=8.4,1.7 \mathrm{~Hz}, 1 \mathrm{H})$, $8.15(\mathrm{~d}, J=8 \mathrm{~Hz}, 1 \mathrm{H}), 7.98-7.92(\mathrm{~m}, 1 \mathrm{H}), 7.76-7.69$ (m, 2H), $7.67-7.61(\mathrm{~m}, 1 \mathrm{H})$, $7.41-7.34(\mathrm{~m}, 2 \mathrm{H}), 6.97(\mathrm{~d}, J=8.2 \mathrm{~Hz}, 1 \mathrm{H}), 2.91(\mathrm{~s}, 3 \mathrm{H}), 2.19(\mathrm{~s}, 3 \mathrm{H}) .{ }^{13} \mathrm{C}\left\{{ }^{1} \mathrm{H}\right\} \mathrm{NMR}$ $\left(100 \mathrm{MHz}, \mathrm{CDCl}_{3}\right): \delta=163.90,158.34,150.71,149.67,145.07,143.51,136.85,136.34$, $134.79,132.01,131.93,131.73,130.56,129.53,128.42$, 126.54, 124.43, 121.45, 119.99, 118.06, 111.94, 24.58, 24.54. FTIR (ATR, $\mathrm{cm}^{-1}$ ): 3049, 2921, 1651, 1592, 1497, 1460, 1383, 1297, 821, 786. HRMS (ESI-MS) m/z calcd for $\mathrm{C}_{23} \mathrm{H}_{18} \mathrm{~N}_{3} \mathrm{O}[\mathrm{M}+\mathrm{H}]^{+} 352.1449$ found 352.1450 .<smiles>COc1ccc2c3cccc(C)c3c(=O)n(-c3cccc4cccnc34)c2n1</smiles>

3-methoxy-7-methyl-5-(quinolin-8-yl)-5H,6H-benzo[c]1,8-naphthyridin-6-one (5c). The title compound was synthesized following the General Procedure A as an ochre solid in $36 \%$ yield $(26.4 \mathrm{mg}, 0.07 \mathrm{mmol}), \mathrm{mp}=204-206{ }^{\circ} \mathrm{C} .{ }^{1} \mathrm{H} \mathrm{NMR}\left(400 \mathrm{MHz}, \mathrm{CDCl}_{3}\right): \delta$ $=8.77(\mathrm{dd}, J=4.2,1.7 \mathrm{~Hz}, 1 \mathrm{H}), 8.40(\mathrm{~d}, J=8.6 \mathrm{~Hz}, 1 \mathrm{H}), 8.23(\mathrm{dd}, J=8.3,1.7 \mathrm{~Hz}, 1 \mathrm{H})$, $8.06(\mathrm{dd}, J=8.1,1.1 \mathrm{~Hz}, 1 \mathrm{H}), 7.94(\mathrm{dd}, J=7.9,1.8 \mathrm{~Hz}, 1 \mathrm{H}), 7.78-7.67$ (m, 2H), $7.66-$ 
$7.59(\mathrm{~m}, 1 \mathrm{H}), 7.38(\mathrm{dd}, J=8.3,4.2 \mathrm{~Hz}, 1 \mathrm{H}), 7.33(\mathrm{dd}, J=7.4,1.9 \mathrm{~Hz}, 1 \mathrm{H}), 6.57(\mathrm{~d}, J=$ $8.6 \mathrm{~Hz}, 1 \mathrm{H}), 3.12(\mathrm{~s}, 3 \mathrm{H}), 2.92(\mathrm{~s}, 3 \mathrm{H}) .{ }^{13} \mathrm{C}\left\{{ }^{1} \mathrm{H}\right\}$ NMR $\left(100 \mathrm{MHz}, \mathrm{CDCl}_{3}\right): \delta=163.98$, 163.01, 150.79, 148.48, 145.00, 143.57, 136.66, 136.23, 135.21, 134.62, 132.12, 130.97, 130.37, 129.38, 128.45, 126.46, 123.61, 121.46, 119.54, 107.74, 105.60, 53.00, 24.56. FTIR $\left(A T R, \mathrm{~cm}^{-1}\right): 3048,2923,1651,1592,1498,1461,1346,1295,1257,1111,824$, 784. HRMS (ESI-MS) $\mathrm{m} / \mathrm{z}$ calcd for $\mathrm{C}_{23} \mathrm{H}_{18} \mathrm{~N}_{3} \mathrm{O}_{2}[\mathrm{M}+\mathrm{H}]^{+} 368.1399$ found 368.1397 .<smiles>Cc1cccc2c(=O)n(-c3cccc4cccnc34)c3nc(F)ccc3c12</smiles>

3-fluoro-7-methyl-5-(quinolin-8-yl)-5H,6H-benzo[c]1,8-naphthyridin-6-one (5d). The title compound was synthesized following the General Procedure A as a white solid in $29 \%$ yield $(20.6 \mathrm{mg}, 0.05 \mathrm{mmol}), \mathrm{mp}=336-338{ }^{\circ} \mathrm{C} .{ }^{1} \mathrm{H} \mathrm{NMR}\left(400 \mathrm{MHz}, \mathrm{CDCl}_{3}\right): \delta=$ $8.74(\mathrm{dd}, J=4.2,1.7 \mathrm{~Hz}, 1 \mathrm{H}), 8.63(\mathrm{t}, 1 \mathrm{H}), 8.25(\mathrm{dd}, J=8.3,1.7 \mathrm{~Hz}, 1 \mathrm{H}), 8.12(\mathrm{~d}, J=8.1$ $\mathrm{Hz}, 1 \mathrm{H}), 8.02-7.96$ (m, 1H), $7.77-7.66$ (m, 3H), $7.45-7.37$ (m, 2H), 6.76 (dd, $J=8.5$, $3.1 \mathrm{~Hz}, 1 \mathrm{H}), 2.90(\mathrm{~s}, 3 \mathrm{H}) \cdot{ }^{13} \mathrm{C}\left\{{ }^{1} \mathrm{H}\right\} \mathrm{NMR}\left(100 \mathrm{MHz}, \mathrm{CDCl}_{3}\right): \delta=163.71,162.14(\mathrm{~d}, J=$ $241.9 \mathrm{~Hz}), 150.97,149.00,144.70,143.87,136.94(\mathrm{~d}, J=8.8 \mathrm{~Hz}), 136.49,135.67$, 134.03, 132.40 (d, $J=10.4 \mathrm{~Hz}), 130.39,129.72,129.14$, 126.67, 124.24, 121.75, 120.10, $112.37(\mathrm{~d}, J=4.6 \mathrm{~Hz}), 103.69,103.31,24.49 .{ }^{19} \mathrm{~F}$ NMR $\left(376 \mathrm{MHz}, \mathrm{CDCl}_{3}\right): \delta=-65.85$ (dd, $J=7.6,3.0 \mathrm{~Hz}$ ). FTIR (ATR, $\mathrm{cm}^{-1}$ ): 3050, 2923, 1652, 1588, 1498, 1454, 1292, 1224, 823, 798, 786. HRMS (ESI-MS) $\mathrm{m} / \mathrm{z}$ calcd for $\mathrm{C}_{22} \mathrm{H}_{15} \mathrm{FN}_{3} \mathrm{O}[\mathrm{M}+\mathrm{H}]^{+} 356.1199$ found 356.1198 . 
<smiles>Cc1cccc2c1c(=O)n(-c1cccc3cccnc13)c1nc(Cl)ccc21</smiles>

3-chloro-7-methyl-5-(quinolin-8-yl)-5H,6H-benzo[c]1,8-naphthyridin-6-one (5e). The title compound was synthesized following the General Procedure A as a white solid in $27 \%$ (20.0 mg, $0.05 \mathrm{mmol}), \mathrm{mp}=326-328{ }^{\circ} \mathrm{C} .{ }^{1} \mathrm{H}$ NMR $\left(400 \mathrm{MHz}, \mathrm{CDCl}_{3}\right): \delta=8.74$ $(\mathrm{dd}, J=4.4,1.6 \mathrm{~Hz}, 1 \mathrm{H}), 8.47(\mathrm{~d}, J=8.2 \mathrm{~Hz}, 1 \mathrm{H}), 8.25(\mathrm{dd}, J=8.4,1.7 \mathrm{~Hz}, 1 \mathrm{H}), 8.14(\mathrm{~d}$, $J=8.2 \mathrm{~Hz}, 1 \mathrm{H}), 8.01-7.96(\mathrm{~m}, 1 \mathrm{H}), 7.77-7.71(\mathrm{~m}, 2 \mathrm{H}), 7.69(\mathrm{t}, J=7.8 \mathrm{~Hz}, 1 \mathrm{H}), 7.45$ $(\mathrm{d}, J=7.5 \mathrm{~Hz}, 1 \mathrm{H}), 7.40(\mathrm{dd}, J=8.3,4.2 \mathrm{~Hz}, 1 \mathrm{H}), 7.14(\mathrm{~d}, J=8.2 \mathrm{~Hz}, 1 \mathrm{H}), 2.90(\mathrm{~s}, 3 \mathrm{H})$. ${ }^{13} \mathrm{C}\left\{{ }^{1} \mathrm{H}\right\} \mathrm{NMR}\left(100 \mathrm{MHz}, \mathrm{CDCl}_{3}\right): \delta=163.53,150.90,149.93,144.72,143.88,136.47$, $135.79,134.10,133.83,132.81,132.40,130.48,129.65,129.00,126.63,124.59,121.68$, 120.32, 118.48, 113.48, 24.48. FTIR (ATR, $\mathrm{cm}^{-1}$ ): 3093, 2924, 1661, 1599, 1579, 1498, 1439, 1391, 1286, 1128, 1076, 867, 816, 788. HRMS (ESI-MS) m/z calcd for $\mathrm{C}_{22} \mathrm{H}_{15} \mathrm{ClN}_{3} \mathrm{O}[\mathrm{M}+\mathrm{H}]^{+} 372.0903$ found 372.0900 .<smiles>Cc1cccc2c1c(=O)n(-c1cccc3cccnc13)c1ncc(Br)cc21</smiles>

2-bromo-7-methyl-5-(quinolin-8-yl)-5H,6H-benzo[c]1,8-naphthyridin-6-one (5s). The title compound was synthesized following the General Procedure A. White solid in $40 \%$ ( $33.3 \mathrm{mg}, 0.08 \mathrm{mmol}), \mathrm{mp}=294-296{ }^{\circ} \mathrm{C} .{ }^{1} \mathrm{H} \mathrm{NMR}\left(400 \mathrm{MHz}, \mathrm{CDCl}_{3}\right): \delta=8.74(\mathrm{dd}, J=$ $4.2,1.7 \mathrm{~Hz}, 1 \mathrm{H}), 8.65(\mathrm{~d}, J=2.2 \mathrm{~Hz}, 1 \mathrm{H}), 8.25(\mathrm{dd}, J=8.3,1.7 \mathrm{~Hz}, 1 \mathrm{H}), 8.21(\mathrm{~d}, J=2.2$ $\mathrm{Hz}, 1 \mathrm{H}), 8.16$ (d, $J=8.5 \mathrm{~Hz}, 1 \mathrm{H}), 7.99$ (dt, $J=7.4,3.7 \mathrm{~Hz}, 1 \mathrm{H}), 7.78-7.67$ (m, 3H), $7.47(\mathrm{~d}, J=7.4 \mathrm{~Hz}, 1 \mathrm{H}), 7.40(\mathrm{dd}, J=8.3,4.2 \mathrm{~Hz}, 1 \mathrm{H}), 2.90(\mathrm{~s}, 3 \mathrm{H}) .{ }^{13} \mathrm{C}\left\{{ }^{1} \mathrm{H}\right\} \mathrm{NMR}(100$ $\left.\mathrm{MHz}, \mathrm{CDCl}_{3}\right): \delta=163.41,151.03,149.35,148.99,144.70,143.91,136.52,136.11$, $133.85,133.35,133.27,132.40,130.31,129.71,129.10,126.71,124.87,121.80,120.48$, 
116.48, 113.69, 24.45. FTIR (ATR, $\mathrm{cm}^{-1}$ ): 3048, 2928, 1665, 1597, 1499, 1465, 1388, 1288, 964, 827, 795, 689. HRMS (ESI-MS) m/z calcd for $\mathrm{C}_{22} \mathrm{H}_{15} \mathrm{BrN}_{3} \mathrm{O}[\mathrm{M}+\mathrm{H}]^{+}$ 416.0398 found 416.0396 .<smiles>Cc1cnc2c(c1)c1cccc(C)c1c(=O)n2-c1cccc2cccnc12</smiles>

2,7-dimethyl-5-(quinolin-8-yl)-5H,6H-benzo[c]1,8-naphthyridin-6-one (5f). The title compound was synthesized following the General Procedure A as a light brown solid in $70 \%$ yield (49.2 mg, $0.14 \mathrm{mmol}), \mathrm{mp}=276-278{ }^{\circ} \mathrm{C} .{ }^{1} \mathrm{H} \mathrm{NMR}\left(400 \mathrm{MHz}, \mathrm{CDCl}_{3}\right): \delta=$ $8.76(\mathrm{dd}, J=4.1,1.5 \mathrm{~Hz}, 1 \mathrm{H}), 8.37-8.32(\mathrm{~m}, 1 \mathrm{H}), 8.24(\mathrm{dd}, J=8.3,1.5 \mathrm{~Hz}, 1 \mathrm{H}), 8.19$ $(\mathrm{d}, J=8.1 \mathrm{~Hz}, 1 \mathrm{H}), 8.07-8.02(\mathrm{~m}, 1 \mathrm{H}), 7.98(\mathrm{dd}, J=6.8,2.8 \mathrm{~Hz}, 1 \mathrm{H}), 7.79-7.72(\mathrm{~m}$, $2 \mathrm{H}), 7.64(\mathrm{t}, J=7.8 \mathrm{~Hz}, 1 \mathrm{H}), 7.44-7.35(\mathrm{~m}, 2 \mathrm{H}), 2.92(\mathrm{~s}, 3 \mathrm{H}), 2.36(\mathrm{~s}, 3 \mathrm{H}) .{ }^{13} \mathrm{C}\left\{{ }^{1} \mathrm{H}\right\}$ NMR (100 MHz, $\left.\mathrm{CDCl}_{3}\right): \delta=163.63,150.87,149.29,148.54,144.88,143.51,136.69$, 136.46, 134.39, 132.43, 131.99, 131.93, 130.40, 129.68, 128.81, 127.36, 126.70, 124.74, 121.62, 120.24, 114.28, 24.52, 18.06. FTIR (ATR, $\mathrm{cm}^{-1}$ ): 3050, 2920, 1652, 1599, 1479, 1301, 805, 782. HRMS (ESI-MS) m/z calcd for $\mathrm{C}_{23} \mathrm{H}_{18} \mathrm{~N}_{3} \mathrm{O}[\mathrm{M}+\mathrm{H}]^{+} 352.1449$ found 352.1450 .<smiles>Cc1cccc2c1c(=O)n(-c1cccc3cccnc13)c1ncccc21</smiles>

8-methyl-6-(quinolin-8-yl)-6,7-dihydro-5,6-diazatetraphen-7-one (5h). The title compound was synthesized following the General Procedure A as a white solid in 51\% yield (39.5 mg, $0.10 \mathrm{mmol}), \mathrm{mp}=332-334{ }^{\circ} \mathrm{C} .{ }^{1} \mathrm{H}$ NMR $\left(400 \mathrm{MHz}, \mathrm{CDCl}_{3}\right): \delta=8.99$ 
(s, $1 \mathrm{H}), 8.71(\mathrm{dd}, J=4.2,1.7 \mathrm{~Hz}, 1 \mathrm{H}), 8.38(\mathrm{~d}, J=8.0 \mathrm{~Hz}, 1 \mathrm{H}), 8.26(\mathrm{dd}, J=8.3,1.7 \mathrm{~Hz}$, $1 \mathrm{H}), 8.01(\mathrm{dd}, J=7.9,1.8 \mathrm{~Hz}, 1 \mathrm{H}), 7.88(\mathrm{dd}, J=8.1,1.3 \mathrm{~Hz}, 1 \mathrm{H}), 7.83-7.74(\mathrm{~m}, 2 \mathrm{H})$, $7.70(\mathrm{t}, J=7.8 \mathrm{~Hz}, 1 \mathrm{H}), 7.53-7.43(\mathrm{~m}, 3 \mathrm{H}), 7.41-7.34(\mathrm{~m}, 2 \mathrm{H}), 2.92(\mathrm{~s}, 3 \mathrm{H}) .{ }^{13} \mathrm{C}\left\{{ }^{1} \mathrm{H}\right\}$ NMR $\left(100 \mathrm{MHz}, \mathrm{CDCl}_{3}\right): \delta=164.11,150.80,149.81,147.20,145.02,143.81,136.86$, 136.41, 134.33, 133.01, 132.27, 131.62, 130.69, 130.18, 129.67, 128.59, 128.37, 127.91, 126.68, 125.29, 124.95, 124.67, 121.55, 120.66, 115.99, 24.54. FTIR (ATR, $\mathrm{cm}^{-1}$ ): 3049, 2923, 1652, 1599, 1499, 1280, 881, 803. HRMS (ESI-MS) m/z calcd for $\mathrm{C}_{26} \mathrm{H}_{18} \mathrm{~N}_{3} \mathrm{O}$ [M $+\mathrm{H}]^{+} 388.1449$ found 388.1450 .

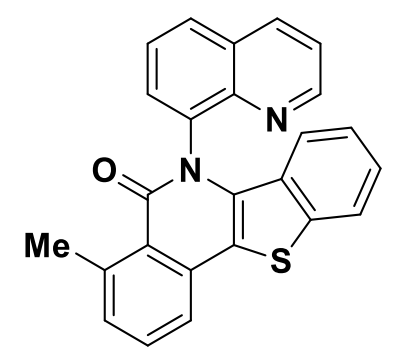

8-methyl-6-(quinolin-8-yl)-6H,7H-benzothieno[3,2-c]isoquinolin-7-one (5i). The title compound was synthesized following the General Procedure $\mathrm{C}$ as a yellow solid in $44 \%$ yield $(34.5 \mathrm{mg}, 0.08 \mathrm{mmol}), \mathrm{mp}=240-242{ }^{\circ} \mathrm{C} .{ }^{1} \mathrm{H}$ NMR $\left(400 \mathrm{MHz}, \mathrm{CDCl}_{3}\right): \delta=8.78$ $(\mathrm{dd}, J=4.2,1.7 \mathrm{~Hz}, 1 \mathrm{H}), 8.30(\mathrm{dd}, J=8.4,1.7 \mathrm{~Hz}, 1 \mathrm{H}), 8.12(\mathrm{dd}, J=8.2,1.4 \mathrm{~Hz}, 1 \mathrm{H})$, $7.88(\mathrm{dd}, J=7.3,1.4 \mathrm{~Hz}, 1 \mathrm{H}), 7.82-7.73(\mathrm{~m}, 2 \mathrm{H}), 7.70(\mathrm{~d}, J=7.7 \mathrm{~Hz}, 1 \mathrm{H}), 7.62(\mathrm{t}, J=$ $7.7 \mathrm{~Hz}, 1 \mathrm{H}), 7.41(\mathrm{dd}, J=8.3,4.2 \mathrm{~Hz}, 1 \mathrm{H}), 7.34(\mathrm{~d}, J=7.3 \mathrm{~Hz}, 1 \mathrm{H}), 7.15$ (ddd, $J=8.1$, 7.1, $1.0 \mathrm{~Hz}, 1 \mathrm{H}), 6.73(\mathrm{ddd}, J=8.4,7.1,1.1 \mathrm{~Hz}, 1 \mathrm{H}), 5.68(\mathrm{dd}, J=8.0,1.0 \mathrm{~Hz}, 1 \mathrm{H}, 2.94$ (s, $3 \mathrm{H}) .{ }^{13} \mathrm{C}\left\{{ }^{1} \mathrm{H}\right\} \mathrm{NMR}\left(100 \mathrm{MHz}, \mathrm{CDCl}_{3}\right): \delta=163.73,151.61,145.05,143.58,138.36$, 137.37, 136.31, 134.87, 133.71, 132.34, 130.86, 130.46, 129.68, 129.45, 126.65, 125.25, 124.04, 123.15, 123.01, 122.48, 122.10, 121.75, 117.75, 24.12. FTIR (ATR, $\mathrm{cm}^{-1}$ ): 3067, 2922, 1645, 1597, 1497, 1388, 1294, 905, 814, 727. HRMS (ESI-MS) m/z calcd for $\mathrm{C}_{25} \mathrm{H}_{17} \mathrm{~N}_{2} \mathrm{OS}[\mathrm{M}+\mathrm{H}]^{+} 393.1061$ found 393.1061 . 


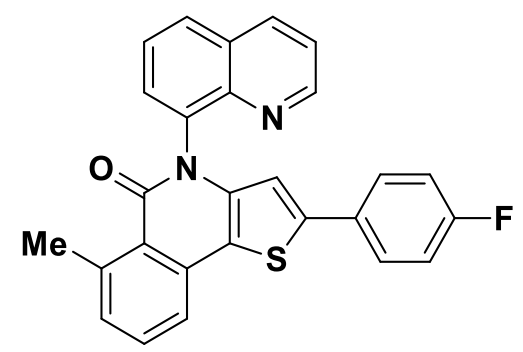

2-(4-fluorophenyl)-6-methyl-4-(quinolin-8-yl)-4H,5H-thieno[3,2-c]isoquinolin-5-one

(5j). The title compound was synthesized following the General Procedure B as a yellow solid in $23 \%$ yield $(20.1 \mathrm{mg}, 0.04 \mathrm{mmol}), \mathrm{mp}=250-252{ }^{\circ} \mathrm{C} .{ }^{1} \mathrm{H}$ NMR $(400 \mathrm{MHz}$, $\left.\mathrm{CDCl}_{3}\right): \delta=8.87(\mathrm{dd}, J=4.2,1.7 \mathrm{~Hz}, 1 \mathrm{H}), 8.28(\mathrm{dd}, J=8.3,1.8 \mathrm{~Hz}, 1 \mathrm{H}), 8.03(\mathrm{dd}, J=$ 8.2, $1.5 \mathrm{~Hz}, 1 \mathrm{H}), 7.84$ (dd, $J=7.3,1.5 \mathrm{~Hz}, 1 \mathrm{H}), 7.75$ (dd, $J=8.2,7.3 \mathrm{~Hz}, 1 \mathrm{H}), 7.62$ (dd, $J$ $=8.0,1.4 \mathrm{~Hz}, 1 \mathrm{H}), 7.56(\mathrm{t}, J=7.6 \mathrm{~Hz}, 1 \mathrm{H}), 7.45(\mathrm{dd}, J=8.3,4.2 \mathrm{~Hz}, 1 \mathrm{H}), 7.41-7.36$ $(\mathrm{m}, 2 \mathrm{H}), 7.26(\mathrm{dt}, J=7.3,1.1 \mathrm{~Hz}, 1 \mathrm{H}), 7.02-6.93(\mathrm{~m}, 2 \mathrm{H}), 6.22(\mathrm{~s}, 1 \mathrm{H}), 2.90(\mathrm{~s}, 3 \mathrm{H})$. ${ }^{13} \mathrm{C}\left\{{ }^{1} \mathrm{H}\right\} \mathrm{NMR}\left(100 \mathrm{MHz}, \mathrm{CDCl}_{3}\right): \delta=163.05,162.65(\mathrm{~d}, J=248.0 \mathrm{~Hz}), 151.49,144.31$, $143.75,142.20,141.40,137.04,136.31,134.69$, 132.24, 130.13, 130.03, 129.87 (d, $J=$ $3.4 \mathrm{~Hz}), 129.74,129.41,127.51$ (d, $J=8.1 \mathrm{~Hz}), 126.66,122.44,122.01,120.57,116.72$, $115.83(\mathrm{~d}, J=22.0 \mathrm{~Hz}), 114.18,24.20 .{ }^{19} \mathrm{~F}$ NMR $\left(376 \mathrm{MHz}, \mathrm{CDCl}_{3}\right): \delta=-112.96--$ 113.13 (m). FTIR (ATR, cm ${ }^{-1}$ ): 3067, 2920, 1640, 1598, 1492, 1228, 882, 818, 787. HRMS (ESI-MS) m/z calcd for $\mathrm{C}_{27} \mathrm{H}_{18} \mathrm{FN}_{2} \mathrm{OS}[\mathrm{M}+\mathrm{H}]^{+} 437.1123$ found 437.1124.<smiles>Cc1cccc2c1c(=O)n(-c1cccc3cccnc13)c1ccccc21</smiles>

7-methyl-5-(quinolin-8-yl)-5,6-dihydrophenanthridin-6-one (5j). The title compound was synthesized following the General Procedure B as an off white solid in 68\% (45.8 $\mathrm{mg}, 0.13 \mathrm{mmol}) .{ }^{1} \mathrm{H} \mathrm{NMR}\left(400 \mathrm{MHz}, \mathrm{CDCl}_{3}\right): \delta=8.81(\mathrm{dd}, J=4.2,1.6 \mathrm{~Hz}, 1 \mathrm{H}), 8.36-$ $8.24(\mathrm{~m}, 3 \mathrm{H}), 8.02(\mathrm{dd}, J=7.3,2.4 \mathrm{~Hz}, 1 \mathrm{H}), 7.82-7.73(\mathrm{~m}, 2 \mathrm{H}), 7.65(\mathrm{t}, J=7.8 \mathrm{~Hz}, 1 \mathrm{H})$, $7.41(\mathrm{dt}, J=8.6,4.4 \mathrm{~Hz}, 2 \mathrm{H}), 7.18(\mathrm{dtd}, J=19.0,7.3,1.4 \mathrm{~Hz}, 2 \mathrm{H}), 6.41$ (dd, $J=8.1,1.5$ $\mathrm{Hz}, 1 \mathrm{H}), 2.95$ (s, 3H). ${ }^{13} \mathrm{C}\left\{{ }^{1} \mathrm{H}\right\} \mathrm{NMR}\left(100 \mathrm{MHz}, \mathrm{CDCl}_{3}\right): \delta=162.87,151.40,144.73$, 
$143.18,139.70,136.70,136.41,136.05,131.94,131.80,130.76,129.93,129.25,128.96$, $126.94,124.51,123.49,122.20,121.98,120.25,119.32,116.51,24.64$. The spectral data are consistent with those reported in the literature. ${ }^{65}$<smiles>Cc1cccc2c(=O)n(-c3cccc4cccnc34)c3ccc(F)cc3c12</smiles>

2-fluoro-7-methyl-5-(quinolin-8-yl)-5,6-dihydrophenanthridin-6-one (5k). The title compound was synthesized following the General Procedure B as an ochre solid in $62 \%$ yield $(43.8 \mathrm{mg}, 0.12 \mathrm{mmol}), \mathrm{mp}=237-239{ }^{\circ} \mathrm{C} .{ }^{1} \mathrm{H} \mathrm{NMR}\left(400 \mathrm{MHz}, \mathrm{CDCl}_{3}\right): \delta=8.81$ $(\mathrm{dd}, J=4.2,1.7 \mathrm{~Hz}, 1 \mathrm{H}), 8.28(\mathrm{dd}, J=8.3,1.7 \mathrm{~Hz}, 1 \mathrm{H}), 8.15(\mathrm{~d}, J=8.2 \mathrm{~Hz}, 1 \mathrm{H}), 8.06-$ $8.00(\mathrm{~m}, 1 \mathrm{H}), 7.96(\mathrm{dd}, J=10.2,2.8 \mathrm{~Hz}, 1 \mathrm{H}), 7.79-7.73(\mathrm{~m}, 2 \mathrm{H}), 7.67$ (t, $J=7.8 \mathrm{~Hz}$, $1 \mathrm{H}), 7.43(\mathrm{dd}, J=8.3,4.3 \mathrm{~Hz}, 2 \mathrm{H}), 6.87$ (ddd, $J=9.3,7.6,2.8 \mathrm{~Hz}, 1 \mathrm{H}), 6.34(\mathrm{dd}, J=9.2$, $4.9 \mathrm{~Hz}, 1 \mathrm{H}), 2.92(\mathrm{~s}, 3 \mathrm{H}) .{ }^{13} \mathrm{C}\left\{{ }^{1} \mathrm{H}\right\} \mathrm{NMR}\left(100 \mathrm{MHz}, \mathrm{CDCl}_{3}\right): \delta=162.57,158.48(\mathrm{~d}, J=$ $240.3 \mathrm{~Hz}), 151.54,144.70,143.48,136.62,136.51,136.21$ (d, $J=1.8 \mathrm{~Hz}), 135.19$ (d, $J=$ $2.8 \mathrm{~Hz}), 132.52,132.10,130.83,130.04,129.46,127.03,124.75,122.12,120.61$ (d, $J=$ $7.7 \mathrm{~Hz}), 120.51,118.03$ (d, $J=8.2 \mathrm{~Hz}), 116.42(\mathrm{~d}, J=23.6 \mathrm{~Hz}), 109.39$ (d, $J=24.1 \mathrm{~Hz})$, 24.60. ${ }^{19} \mathrm{~F}$ NMR $\left(376 \mathrm{MHz}, \mathrm{CDCl}_{3}\right.$ ): $\delta=-121.35$ (ddd, $J=10.2,7.5,4.8 \mathrm{~Hz}$ ). FTIR $\left(\right.$ ATR, $\mathrm{cm}^{-1}$ ): 3073, 2924, 1652, 1592, 1481, 1396, 1303, 1205, 961, 828, 799, 758. HRMS (ESI-MS) m/z calcd for $\mathrm{C}_{23} \mathrm{H}_{16} \mathrm{FN}_{2} \mathrm{O}[\mathrm{M}+\mathrm{H}]^{+} 355.1246$ found 355.1245 .<smiles>Cc1ccc2c(c1)c1cccc(C)c1c(=O)n2-c1cccc2cccnc12</smiles>

2,7-dimethyl-5-(quinolin-8-yl)-5,6-dihydrophenanthridin-6-one (51). The title compound was synthesized following the General Procedure B as a light brown solid in 
$56 \%$ yield (39.2 mg, $0.11 \mathrm{mmol}) .{ }^{1} \mathrm{H}$ NMR (400 MHz, $\left.\mathrm{CDCl}_{3}\right): \delta=8.81(\mathrm{dd}, J=4.1,1.5$ $\mathrm{Hz}, 1 \mathrm{H}), 8.32-8.24(\mathrm{~m}, 2 \mathrm{H}), 8.12(\mathrm{~s}, 1 \mathrm{H}), 8.01$ (dd, $J=6.4,3.3 \mathrm{~Hz}, 1 \mathrm{H}), 7.79-7.72(\mathrm{~m}$, 2H), $7.65(\mathrm{t}, J=7.8 \mathrm{~Hz}, 1 \mathrm{H}), 7.45-7.36(\mathrm{~m}, 2 \mathrm{H}), 6.97(\mathrm{dd}, J=8.5,1.7 \mathrm{~Hz}, 1 \mathrm{H}), 6.28(\mathrm{~d}$, $J=8.5 \mathrm{~Hz}, 1 \mathrm{H}), 2.93(\mathrm{~s}, 3 \mathrm{H}), 2.42(\mathrm{~s}, 3 \mathrm{H}) .{ }^{13} \mathrm{C}\left\{{ }^{1} \mathrm{H}\right\} \mathrm{NMR}\left(100 \mathrm{MHz}, \mathrm{CDCl}_{3}\right): \delta=$ 162.82 , 151.44, 144.81, 143.25, 137.72, 136.89, 136.44, 136.05, 131.86, 131.73, 131.51, $130.81,130.09,129.97,129.21,126.98,124.64,123.61,121.98,120.24,119.16,116.44$, $24.70,21.17$. The spectral data are consistent with those reported in the literature. ${ }^{65}$<smiles>Cc1cccc2c1c(=O)n(-c1cccc3cccnc13)c1cc(Br)ccc21</smiles>

3-bromo-7-methyl-5-(quinolin-8-yl)-5,6-dihydrophenanthridin-6-one (5m). The title compound was synthesized following the General Procedure B as an off white solid in $36 \%$ yield $(29.7 \mathrm{mg}, 0.07 \mathrm{mmol}), \mathrm{mp}=253-255{ }^{\circ} \mathrm{C} .{ }^{1} \mathrm{H} \mathrm{NMR}\left(400 \mathrm{MHz}, \mathrm{CDCl}_{3}\right): \delta=$ $8.81(\mathrm{dd}, J=4.2,1.7 \mathrm{~Hz}, 1 \mathrm{H}), 8.29(\mathrm{dd}, J=8.3,1.7 \mathrm{~Hz}, 1 \mathrm{H}), 8.20(\mathrm{~d}, J=8.2 \mathrm{~Hz}, 1 \mathrm{H})$, $8.15(\mathrm{~d}, J=8.7 \mathrm{~Hz}, 1 \mathrm{H}), 8.04(\mathrm{dd}, J=7.3,2.4 \mathrm{~Hz}, 1 \mathrm{H}), 7.80-7.72(\mathrm{~m}, 2 \mathrm{H}), 7.65$ (t, $J=$ $7.8 \mathrm{~Hz}, 1 \mathrm{H}), 7.45(\mathrm{dd}, J=8.3,4.2 \mathrm{~Hz}, 1 \mathrm{H}), 7.40$ (d, $J=7.4 \mathrm{~Hz}, 1 \mathrm{H}), 7.31(\mathrm{dd}, J=8.7,1.9$ $\mathrm{Hz}, 1 \mathrm{H}), 6.53(\mathrm{~d}, J=1.9 \mathrm{~Hz}, 1 \mathrm{H}), 2.89(\mathrm{~s}, 3 \mathrm{H}) .{ }^{13} \mathrm{C}\left\{{ }^{1} \mathrm{H}\right\} \mathrm{NMR}\left(100 \mathrm{MHz}, \mathrm{CDCl}_{3}\right): \delta=$ $162.73,151.63,144.57,143.49,140.77,136.59,136.09,135.45,132.25,132.24,130.77$, 130.09 , 129.72, 127.04, 125.40, 125.02, 124.42, 123.03, 122.23, 120.22, 119.11, 118.43, 24.60. FTIR (ATR, $\mathrm{cm}^{-1}$ ): 3060, 2921, 1651, 1594, 1499, 1464, 1387, 1301, 802, 783, 685. HRMS (ESI-MS) m/z calcd for $\mathrm{C}_{23} \mathrm{H}_{16} \mathrm{BrN}_{2} \mathrm{O}[\mathrm{M}+\mathrm{H}]^{+} 415.0445$ found 415.0447 . 
<smiles>Cc1cccc2c1c(=O)n(-c1cccc3cccnc13)c1cc(F)ccc21</smiles>

3-fluoro-7-methyl-5-(quinolin-8-yl)-5,6-dihydrophenanthridin-6-one (5n). The title compound was synthesized following the General Procedure B as an off white solid in $68 \%$ ( $48.7 \mathrm{mg}, 0.13 \mathrm{mmol}), \mathrm{mp}=233-235{ }^{\circ} \mathrm{C} .{ }^{1} \mathrm{H}$ NMR $\left(400 \mathrm{MHz}, \mathrm{CDCl}_{3}\right): \delta=8.81$ $(\mathrm{dd}, J=4.2,1.7 \mathrm{~Hz}, 1 \mathrm{H}), 8.32-8.23(\mathrm{~m}, 2 \mathrm{H}), 8.16(\mathrm{~d}, J=8.2 \mathrm{~Hz}, 1 \mathrm{H}), 8.08-8.00(\mathrm{~m}$, 1H), $7.79-7.74(\mathrm{~m}, 2 \mathrm{H}), 7.64(\mathrm{t}, J=7.8 \mathrm{~Hz}, 1 \mathrm{H}), 7.44(\mathrm{dd}, J=8.3,4.2 \mathrm{~Hz}, 1 \mathrm{H}), 7.37$ (d, $J=7.4 \mathrm{~Hz}, 1 \mathrm{H}), 6.91(\mathrm{ddd}, J=9.0,7.9,2.6 \mathrm{~Hz}, 1 \mathrm{H}), 6.08(\mathrm{dd}, J=10.8,2.6 \mathrm{~Hz}, 1 \mathrm{H}), 2.91$ (s, 3H). ${ }^{13} \mathrm{C}\left\{{ }^{1} \mathrm{H}\right\}$ NMR $\left(100 \mathrm{MHz}, \mathrm{CDCl}_{3}\right): \delta=163.04(\mathrm{~d}, J=246.9 \mathrm{~Hz}), 162.99,151.56$, 144.49, 143.38, 141.22 (d, $J=10.7 \mathrm{~Hz}), 136.55,136.28,135.65,132.21,131.64,130.69$, 130.06, 129.63, 127.00, 125.48 (d, $J=9.8 \mathrm{~Hz}), 123.89$ (d, $J=1.2 \mathrm{~Hz}), 122.17,120.12$, $115.85(\mathrm{~d}, J=2.5 \mathrm{~Hz}), 109.89(\mathrm{~d}, J=22.6 \mathrm{~Hz}), 103.29$ (d, $J=26.9 \mathrm{~Hz}), 24.60 .{ }^{19} \mathrm{~F} \mathrm{NMR}$ $\left(376 \mathrm{MHz}, \mathrm{CDCl}_{3}\right): \delta=-111.14--111.28(\mathrm{~m})$. FTIR (ATR, $\mathrm{cm}^{-1}$ ): 3067, 2967, 1654, $1598,1459,1388,1301,1144,823,784$. HRMS (ESI-MS) m/z calcd for $\mathrm{C}_{23} \mathrm{H}_{16} \mathrm{FN}_{2} \mathrm{O}[\mathrm{M}$ $+\mathrm{H}]^{+} 355.1246$ found 355.1246 .<smiles>Cc1ccc2c3cccc(C)c3c(=O)n(-c3cccc4cccnc34)c2c1</smiles>

3,7-dimethyl-5-(quinolin-8-yl)-5,6-dihydrophenanthridin-6-one (5o). The title compound was synthesized following the General Procedure B as a light brown solid in 
$50 \%$ yield $(35.1 \mathrm{mg}, 0.10 \mathrm{mmol}), \mathrm{mp}=218-220{ }^{\circ} \mathrm{C} .{ }^{1} \mathrm{H} \mathrm{NMR}\left(400 \mathrm{MHz}, \mathrm{CDCl}_{3}\right): \delta=$ $8.82(\mathrm{dd}, J=4.2,1.7 \mathrm{~Hz}, 1 \mathrm{H}), 8.28(\mathrm{dd}, J=8.3,1.7 \mathrm{~Hz}, 1 \mathrm{H}), 8.24(\mathrm{~d}, J=8.2 \mathrm{~Hz}, 1 \mathrm{H})$, $8.19(\mathrm{~d}, J=8.3 \mathrm{~Hz}, 1 \mathrm{H}), 8.07-7.98(\mathrm{~m}, 1 \mathrm{H}), 7.82-7.73(\mathrm{~m}, 2 \mathrm{H}), 7.63(\mathrm{t}, J=7.8 \mathrm{~Hz}$, 1H), $7.43(\mathrm{dd}, J=8.3,4.2 \mathrm{~Hz}, 1 \mathrm{H}), 7.35(\mathrm{~d}, J=7.9 \mathrm{~Hz}, 1 \mathrm{H}), 7.02(\mathrm{~d}, J=8 \mathrm{~Hz}, 1 \mathrm{H}), 6.18$ (s, 1H), 2.91 (s, 3H), 2.15 (s, 3H). ${ }^{13} \mathrm{C}\left\{{ }^{1} \mathrm{H}\right\} \mathrm{NMR}\left(100 \mathrm{MHz}, \mathrm{CDCl}_{3}\right): \delta=163.08,151.50$, 144.87, 143.21, 139.75, 139.34, 136.89, 136.49, 136.27, 131.94, 131.36, 130.82, 129.98, 129.26, 127.00, 124.18, 123.61, 123.47, 122.03, 120.05, 117.02, 116.64, 24.67, 21.70. FTIR (ATR, $\mathrm{cm}^{-1}$ ): 3067, 2920, 1646, 1598, 1498, 1462, 1390, 1300, 822, 788. HRMS (ESI-MS) $\mathrm{m} / \mathrm{z}$ calcd for $\mathrm{C}_{24} \mathrm{H}_{19} \mathrm{~N}_{2} \mathrm{O}[\mathrm{M}+\mathrm{H}]^{+} 351.1497$ found 351.1498 .<smiles>Cc1cc(C)c2c(=O)n(-c3cccc4cccnc34)c3ncccc3c2c1</smiles>

7,9-dimethyl-5-(quinolin-8-yl)-5H,6H-benzo[c]1,8-naphthyridin-6-one (5q): The title compound was synthesized following the General Procedure A as a merlot solid in 75\% yield $(53.2 \mathrm{mg}, 0.15 \mathrm{mmol}), \mathrm{mp}=294-296{ }^{\circ} \mathrm{C} .{ }^{1} \mathrm{H} \mathrm{NMR}\left(400 \mathrm{MHz}, \mathrm{CDCl}_{3}\right): \delta=8.75$ $(\mathrm{dd}, J=4.2,1.7 \mathrm{~Hz}, 1 \mathrm{H}), 8.52(\mathrm{dd}, J=8.1,1.7 \mathrm{~Hz}, 1 \mathrm{H}), 8.23(\mathrm{dd}, J=8.3,1.7 \mathrm{~Hz}, 1 \mathrm{H})$, $8.19(\mathrm{dd}, J=4.6,1.7 \mathrm{~Hz}, 1 \mathrm{H}), 8.00-7.94(\mathrm{~m}, 2 \mathrm{H}), 7.79-7.70(\mathrm{~m}, 2 \mathrm{H}), 7.37$ (dd, $J=8.3$, $4.2 \mathrm{~Hz}, 1 \mathrm{H}), 7.25$ (s, 1H), 7.09 (dd, $J=8.0,4.7 \mathrm{~Hz}, 1 \mathrm{H}), 2.88$ (s, 3H), 2.52 (s, 3H). ${ }^{13} \mathrm{C}\left\{{ }^{1} \mathrm{H}\right\}$ NMR $\left(100 \mathrm{MHz}, \mathrm{CDCl}_{3}\right): \delta=163.70,150.88,150.50,148.69,144.90,143.36$, $142.54,136.70,136.45,134.48,133.90,131.59,130.42,129.67,128.81,126.70,122.38$, 121.64, 120.47, 118.09, 114.83, 24.31, 21.94. FTIR (ATR, $\left.\mathrm{cm}^{-1}\right): 3050,2922,1645$, $1598,1499,1464,1392,1300,823,789$. HRMS (ESI-MS) $\mathrm{m} / \mathrm{z}$ calcd for $\mathrm{C}_{23} \mathrm{H}_{18} \mathrm{~N}_{3} \mathrm{O}[\mathrm{M}$ $+\mathrm{H}]^{+} 352.1449$ found 352.1447 . 
<smiles>COc1cc(C)c2c(=O)n(-c3cccc4cccnc34)c3ncccc3c2c1</smiles>

9-methoxy-7-methyl-5-(quinolin-8-yl)-5H,6H-benzo[c]1,8-naphthyridin-6-one (5r).

The title compound was synthesized following the General Procedure A as a merlot solid in $71 \%$ yield $(52.2 \mathrm{mg}, 0.14 \mathrm{mmol}), \mathrm{mp}=303-305{ }^{\circ} \mathrm{C} .{ }^{1} \mathrm{H} \mathrm{NMR}\left(400 \mathrm{MHz}, \mathrm{CDCl}_{3}\right): \delta$ $=8.75(\mathrm{dd}, J=4.2,1.7 \mathrm{~Hz}, 1 \mathrm{H}), 8.48(\mathrm{dd}, J=8.0,1.6 \mathrm{~Hz}, 1 \mathrm{H}), 8.27-8.19(\mathrm{~m}, 2 \mathrm{H}), 7.97$ $(\mathrm{dt}, J=7.4,3.7 \mathrm{~Hz}, 1 \mathrm{H}), 7.77-7.71(\mathrm{~m}, 2 \mathrm{H}), 7.59(\mathrm{~d}, J=2.5 \mathrm{~Hz}, 1 \mathrm{H}), 7.38(\mathrm{dd}, J=8.3$, $4.2 \mathrm{~Hz}, 1 \mathrm{H}), 7.11$ (dd, $J=8.0,4.7 \mathrm{~Hz}, 1 \mathrm{H}), 6.99$ (d, $J=1.9 \mathrm{~Hz}, 1 \mathrm{H}), 3.99$ (s, 3H), 2.88 (s, $3 \mathrm{H}) .{ }^{13} \mathrm{C}\left\{{ }^{1} \mathrm{H}\right\} \mathrm{NMR}\left(100 \mathrm{MHz}, \mathrm{CDCl}_{3}\right): \delta=163.41,162.11,150.92,150.67,149.06$, $146.13,144.97,136.71,136.57,136.49$, 131.71, 130.50, 129.70, 128.82, 126.73, 121.65, 119.56, 118.57, 118.00, 114.71, 103.70, 55.61, 24.80. FTIR (ATR, $\mathrm{cm}^{-1}$ ): 3004, 2921, 1652, 1601, 1500, 1452, 1399, 1306, 1291, 1166, 1049, 814, 784. HRMS (ESI-MS) m/z calcd for $\mathrm{C}_{23} \mathrm{H}_{18} \mathrm{~N}_{3} \mathrm{O}_{2}[\mathrm{M}+\mathrm{H}]^{+} 368.1399$ found 368.1396.<smiles>Cc1cc(Cl)cc2c1c(=O)n(-c1cccc3cccnc13)c1ncccc21</smiles>

9-chloro-7-methyl-5-(quinolin-8-yl)-5H,6H-benzo[c]1,8-naphthyridin-6-one (5s). The title compound was synthesized following the General Procedure A as a terra cotta solid in $63 \%$ yield $(46.6 \mathrm{mg}, 0.12 \mathrm{mmol}), \mathrm{mp}=284-286{ }^{\circ} \mathrm{C} .{ }^{1} \mathrm{H} \mathrm{NMR}\left(400 \mathrm{MHz}, \mathrm{CDCl}_{3}\right): \delta$ $=8.75(\mathrm{dd}, J=4.2,1.7 \mathrm{~Hz}, 1 \mathrm{H}), 8.45(\mathrm{dd}, J=8.1,1.7 \mathrm{~Hz}, 1 \mathrm{H}), 8.27-8.20(\mathrm{~m}, 2 \mathrm{H}), 8.15$ $(\mathrm{d}, \mathrm{J}=2.0 \mathrm{~Hz}, 1 \mathrm{H}), 7.99(\mathrm{dd}, J=6.4,3.3 \mathrm{~Hz}, 1 \mathrm{H}), 7.78-7.71(\mathrm{~m}, 2 \mathrm{H}), 7.43-7.35(\mathrm{~m}$, 2H), $7.11(\mathrm{dd}, J=8.0,4.7 \mathrm{~Hz}, 1 \mathrm{H}), 2.89(\mathrm{~s}, 3 \mathrm{H}) .{ }^{13} \mathrm{C}\left\{{ }^{1} \mathrm{H}\right\} \mathrm{NMR}\left(100 \mathrm{MHz}, \mathrm{CDCl}_{3}\right): \delta=$ 
$163.04,150.93,150.47,149.54,145.81,144.69,138.59,136.50,136.25,135.98,132.30$, $131.85,130.34,129.67,128.99,126.68,123.12,121.73,120.14,118.38,113.81,24.35$. FTIR (ATR, $\mathrm{cm}^{-1}$ ): 3067, 2923, 1661, 1592, 1501, 1470, 1396, 1300, 825, 783, 760. HRMS (ESI-MS) m/z calcd for $\mathrm{C}_{22} \mathrm{H}_{15} \mathrm{ClN}_{3} \mathrm{O}[\mathrm{M}+\mathrm{H}]^{+} 372.0903$ found 372.0903.<smiles>COc1ccc2c(c1C)c(=O)n(-c1cccc3cccnc13)c1ncccc21</smiles>

8-methoxy-7-methyl-5-(quinolin-8-yl)-5H,6H-benzo[c]1,8-naphthyridin-6-one (5t). The title compound was synthesized following the General Procedure A as an off white solid in $54 \%$ yield $(40.0 \mathrm{mg}, 0.10 \mathrm{mmol}), \mathrm{mp}=337-339{ }^{\circ} \mathrm{C} .{ }^{1} \mathrm{H}$ NMR $(400 \mathrm{MHz}$, $\left.\mathrm{CDCl}_{3}\right): \delta=8.74(\mathrm{dd}, J=4.2,1.8 \mathrm{~Hz}, 1 \mathrm{H}), 8.48(\mathrm{dd}, J=8.0,1.7 \mathrm{~Hz}, 1 \mathrm{H}), 8.24(\mathrm{dd}, J=$ $8.3,1.7 \mathrm{~Hz}, 1 \mathrm{H}), 8.20(\mathrm{~d}, J=8.9 \mathrm{~Hz}, 1 \mathrm{H}), 8.16(\mathrm{dd}, J=4.7,1.6 \mathrm{~Hz}, 1 \mathrm{H}), 8.00-7.95(\mathrm{~m}$, 1H), $7.77-7.71(\mathrm{~m}, 2 \mathrm{H}), 7.43-7.35(\mathrm{~m}, 2 \mathrm{H}), 7.12(\mathrm{dd}, J=7.9,4.7 \mathrm{~Hz}, 1 \mathrm{H}), 3.97(\mathrm{~s}$, $3 \mathrm{H}), 2.82(\mathrm{~s}, 3 \mathrm{H}) .{ }^{13} \mathrm{C}\left\{{ }^{1} \mathrm{H}\right\} \mathrm{NMR}\left(100 \mathrm{MHz}, \mathrm{CDCl}_{3}\right): \delta=163.85,158.47,150.96,149.80$, $147.78,144.92$, 136.87, 136.49, 131.47, 130.72, 130.42, 129.71, 128.84, 127.17, 126.74, 125.75, 121.67, 120.82, 118.22, 115.93, 115.13, 56.49, 13.90. FTIR (ATR, $\mathrm{cm}^{-1}$ ): 3012, 2936, 1655, 1594, 1495, 1452, 1030, 1274, 1163, 1069, 827, 789. HRMS (ESI-MS) m/z calcd for $\mathrm{C}_{23} \mathrm{H}_{18} \mathrm{~N}_{3} \mathrm{O}_{2}[\mathrm{M}+\mathrm{H}]^{+} 368.1399$ found 368.1397 .<smiles>COc1ccc2c(c1C)c(=O)n(-c1cccc3cccnc13)c1ncccc21</smiles>

7,8-dimethyl-5-(quinolin-8-yl)-5H,6H-benzo[c]1,8-naphthyridin-6-one (5u). The title compound was synthesized following the General Procedure A as an off white solid in 
$70 \%$ yield $(49.0 \mathrm{mg}, 0.13 \mathrm{mmol}), \mathrm{mp}=329-331{ }^{\circ} \mathrm{C} .{ }^{1} \mathrm{H} \mathrm{NMR}\left(400 \mathrm{MHz}, \mathrm{CDCl}_{3}\right): \delta=$ $8.74(\mathrm{dd}, J=4.2,1.7 \mathrm{~Hz}, 1 \mathrm{H}), 8.52(\mathrm{dd}, J=8.0,1.6 \mathrm{~Hz}, 1 \mathrm{H}), 8.24(\mathrm{dd}, J=8.3,1.6 \mathrm{~Hz}$, $1 \mathrm{H}), 8.19(\mathrm{dd}, J=4.7,1.6 \mathrm{~Hz}, 1 \mathrm{H}), 8.11(\mathrm{~d}, J=8.3 \mathrm{~Hz}, 1 \mathrm{H}), 8.02-7.95(\mathrm{~m}, 1 \mathrm{H}), 7.78-$ $7.72(\mathrm{~m}, 2 \mathrm{H}), 7.61(\mathrm{~d}, J=8.2 \mathrm{~Hz}, 1 \mathrm{H}), 7.38(\mathrm{dd}, J=8.3,4.2 \mathrm{~Hz}, 1 \mathrm{H}), 7.12(\mathrm{dd}, J=7.9$, $4.7 \mathrm{~Hz}, 1 \mathrm{H}), 2.86(\mathrm{~s}, 3 \mathrm{H}), 2.48(\mathrm{~s}, 3 \mathrm{H}) .{ }^{13} \mathrm{C}\left\{{ }^{1} \mathrm{H}\right\}$ NMR $\left(100 \mathrm{MHz}, \mathrm{CDCl}_{3}\right): \delta=164.05$, $150.89,150.15,148.42,141.75,138.88,136.87,136.55,136.53,134.56,132.47,131.25$, $130.43,129.70,128.82,126.75,124.71,121.66,119.47,118.16,115.03,21.58,18.53$. FTIR (ATR, cm ${ }^{-1}$ ): 3010, 2970, 1651, 1587, 1497, 1385, 1299, 829, 791. HRMS (ESIMS) $\mathrm{m} / \mathrm{z}$ calcd for $\mathrm{C}_{23} \mathrm{H}_{18} \mathrm{~N}_{3} \mathrm{O}[\mathrm{M}+\mathrm{H}]^{+} 352.1449$ found 352.1447 .<smiles>COc1c(Cl)ccc2c(=O)n(-c3cccc4cccnc34)c3ncccc3c12</smiles>

8-chloro-7-methyl-5-(quinolin-8-yl)-5H,6H-benzo[c]1,8-naphthyridin-6-one (5v). The title compound was synthesized following the General Procedure A as a dark brown solid in $31 \%$ yield $(22.9 \mathrm{mg}, 0.06 \mathrm{mmol}), \mathrm{mp}=344-346{ }^{\circ} \mathrm{C} .{ }^{1} \mathrm{H} \mathrm{NMR}\left(400 \mathrm{MHz}, \mathrm{CDCl}_{3}\right): \delta$ $=8.73(\mathrm{dd}, J=4.1,1.8 \mathrm{~Hz}, 1 \mathrm{H}), 8.52(\mathrm{dd}, J=8.0,1.7 \mathrm{~Hz}, 1 \mathrm{H}), 8.29-8.21(\mathrm{~m}, 2 \mathrm{H}), 8.15$ $(\mathrm{d}, J=8.7 \mathrm{~Hz}, 1 \mathrm{H}), 7.99(\mathrm{dt}, J=7.3,3.7 \mathrm{~Hz}, 1 \mathrm{H}), 7.82(\mathrm{~d}, J=8.7 \mathrm{~Hz}, 1 \mathrm{H}), 7.78-7.72$ (m, 2H), 7.39 (dd, $J=8.3,4.2 \mathrm{~Hz}, 1 \mathrm{H}), 7.17$ (dd, $J=8.0,4.6 \mathrm{~Hz}, 1 \mathrm{H}), 3.01$ (s, 3H). ${ }^{13} \mathrm{C}\left\{{ }^{1} \mathrm{H}\right\}$ NMR $\left(100 \mathrm{MHz}, \mathrm{CDCl}_{3}\right): \delta=163.04,151.02,150.26,149.27,144.72,140.95$, $136.88,136.54,136.47,133.59,133.28,131.63,130.30,129.73,129.04,126.73,126.24$, 121.78, 120.87, 118.45, 114.25, 19.34. FTIR (ATR, $\mathrm{cm}^{-1}$ ): 3050, 2999, 1652, 1587, 1499, $1451,1369,1300,811,786,707$. HRMS (ESI-MS) m/z calcd for $\mathrm{C}_{22} \mathrm{H}_{15} \mathrm{ClN}_{3} \mathrm{O}[\mathrm{M}+\mathrm{H}]^{+}$ 372.0903 found 372.0900 . 
<smiles>O=c1c2ccccc2c2cccnc2n1-c1cccc2cccnc12</smiles>

5-(quinolin-8-yl)-5H,6H-benzo[c]1,8-naphthyridin-6-one (5w). The title compound was synthesized following the General Procedure A as a light red solid in $48 \%$ yield (31.3 mg, $0.09 \mathrm{mmol}), \mathrm{mp}=262-264{ }^{\circ} \mathrm{C} .{ }^{1} \mathrm{H}$ NMR $\left(400 \mathrm{MHz}, \mathrm{CDCl}_{3}\right): \delta=8.74(\mathrm{dd}, J$ $=4.1,1.8 \mathrm{~Hz}, 1 \mathrm{H}), 8.61-8.56(\mathrm{~m}, 2 \mathrm{H}), 8.32(\mathrm{dd}, J=8.3,1.3 \mathrm{~Hz}, 1 \mathrm{H}), 8.29-8.23(\mathrm{~m}$, 2H), $8.00(\mathrm{dd}, J=7.1,2.5 \mathrm{~Hz}, 1 \mathrm{H}), 7.87-7.81(\mathrm{~m}, 1 \mathrm{H}), 7.80-7.72(\mathrm{~m}, 2 \mathrm{H}), 7.69-7.63$ $(\mathrm{m}, 1 \mathrm{H}), 7.38(\mathrm{dd}, J=8.3,4.2 \mathrm{~Hz}, 1 \mathrm{H}), 7.19(\mathrm{dd}, J=7.8,4.6 \mathrm{~Hz}, 1 \mathrm{H}) .{ }^{13} \mathrm{C}\left\{{ }^{1} \mathrm{H}\right\} \mathrm{NMR}$ $\left(100 \mathrm{MHz}, \mathrm{CDCl}_{3}\right): \delta=162.94,150.99,150.39,148.94,144.77,136.47,136.16,133.16$, 132.99 , 131.41, 130.36, 129.64, 129.51, 129.08, 128.85, 126.64, 126.37, 122.08, 121.70, 118.52, 114.88. FTIR (ATR, $\mathrm{cm}^{-1}$ ): 3032, 2999, 1652, 1587, 1499, 1451, 1369, 1300, 811, 785. HRMS (ESI-MS) $\mathrm{m} / \mathrm{z}$ calcd for $\mathrm{C}_{21} \mathrm{H}_{14} \mathrm{~N}_{3} \mathrm{O}[\mathrm{M}+\mathrm{H}]^{+} 324.1136$ found 324.1133 .<smiles>Cc1cncc2c1c(=O)n(-c1cccc3cccnc13)c1ncccc21</smiles>

4-methyl-6-(quinolin-8-yl)-5H,6H-pyrido[4,3-c]1,8-naphthyridin-5-one (5x). The title compound was synthesized following the General Procedure A as a terra cotta solid in $61 \%$ yield (41.4 mg, $0.12 \mathrm{mmol}), \mathrm{mp}=335-337{ }^{\circ} \mathrm{C} .{ }^{1} \mathrm{H} \mathrm{NMR}\left(400 \mathrm{MHz}, \mathrm{CDCl}_{3}\right): \delta=$ $9.61(\mathrm{~s}, 1 \mathrm{H}), 8.73(\mathrm{dd}, J=4.3,1.6 \mathrm{~Hz}, 1 \mathrm{H}), 8.71-8.65(\mathrm{~m}, 2 \mathrm{H}), 8.31-8.23(\mathrm{~m}, 2 \mathrm{H})$, $8.04-7.97(\mathrm{~m}, 1 \mathrm{H}), 7.80-7.72(\mathrm{~m}, 2 \mathrm{H}), 7.41(\mathrm{dd}, J=8.3,4.1 \mathrm{~Hz}, 1 \mathrm{H}), 7.22(\mathrm{dd}, J=7.8$, $4.6 \mathrm{~Hz}, 1 \mathrm{H}), 2.87$ (s, 3H). ${ }^{13} \mathrm{C}\left\{{ }^{1} \mathrm{H}\right\} \mathrm{NMR}\left(100 \mathrm{MHz}, \mathrm{CDCl}_{3}\right): \delta=162.62,151.78,151.08$, 
$150.61,149.83,144.54,143.97,136.55,135.83,135.33,131.30,130.20,129.72,129.67$, 129.26, 127.83, 126.70, 121.88, 118.84, 112.92, 20.25. FTIR (ATR, $\left.\mathrm{cm}^{-1}\right): 3048,2956$, $1659,1593,1448,1407,1298,827,786$. HRMS (ESI-MS) $\mathrm{m} / \mathrm{z}$ calcd for $\mathrm{C}_{21} \mathrm{H}_{15} \mathrm{~N}_{4} \mathrm{O}[\mathrm{M}$ $+\mathrm{H}]^{+} 339.1245$ found 339.1243 . 
$\begin{array}{lllllll}\text { Chapter 4: } & \text { Synthetic Routes to a Possible }\end{array}$ Nickel(II) Metallacycle: Denitrogenation and Decarbonylation

\subsection{Overview}

Nickel complexes have been widely employed as catalyst in $\mathrm{C}-\mathrm{C}$ and $\mathrm{C}$-heteroatom bond transformations in traditional cross-coupling reactions. ${ }^{1}$ While nickel has experienced a revival as an economical and complementary alternative to palladiumcatalyzed methods in recent years, the mechanism of nickel-catalyzed reactions are not fully understood. However, many of these reactions involve a proposed $\mathrm{Ni}$ (II) metallacycle complex to result from $\mathrm{C}-\mathrm{H}$ activation ${ }^{41,72}$ allowing the proposed $\mathrm{Ni}(\mathrm{II})$ metallacycle to react with a coupling partner through either a two electron or single electron pathway. ${ }^{73}$ Despite the ubiquity of these proposed species in catalytic cycles, such a complex has not yet been isolated or shown to be catalytically relevant.

In the revival of nickel catalysis, many groups have begun to isolate and study elusive $\mathrm{Ni}(\mathrm{III})$ and $\mathrm{Ni}(\mathrm{IV})$ dialkyl/aryl complexes. Mirica and Sanford demonstrated that a cleverly designed tetradentate or tridentate nitrogen donor ligand could be used to stabilize an isolable $\mathrm{Ni}(\mathrm{III})$ or $\mathrm{Ni}(\mathrm{IV})$ dialkyl/aryl complex. ${ }^{74}$ More recently, the Love group has reported a $\mathrm{Ni}(\mathrm{II})$ metallacycle from $\mathrm{sp}^{3} \mathrm{C}-\mathrm{H}$ activation of a urea moiety (Scheme 23). ${ }^{75}$ The urea moiety was needed in order to stabilize the Ni(II) metallacycle. In addition, the Love group also showed the metallacycle would react with a small set of coupling partners, but in low yields $(20-48 \%)$. Unfortunately, this urea moiety has not been shown to be catalytically relevant.
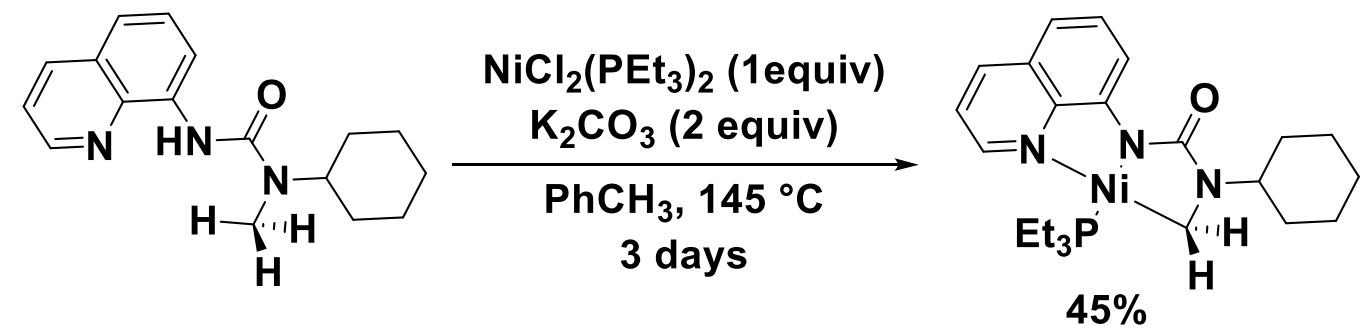

Scheme 23. Ni(II)-mediated $\mathrm{sp}^{3} \mathrm{C}-\mathrm{H}$ activation of a tertiary urea moiety. 
We have recently proposed a Ni(II) metallacycle in our oxidative decarboxylative (hetero)arylation and annulation reactions above in chapters 2 and 3.66,76 Under our reaction conditions, we proposed the $\mathrm{Ni}$ (II) metallacycle intermediate to undergo transmetallation with a silver(I)-aryl and concomitant oxidation to form the desired product. However, in order to study our proposed reaction, we first had to isolate the $\mathrm{Ni}(\mathrm{II})$ intermediate. We then hypothesized the desired metallacycle could be isolated through either denitrogenation or decarbonylation.

Similar to decarboxylative coupling reactions discussed above, decarbonylation reactions represent another approach in creating a metal-C bond. This involves the insertion of a transition-metal into an aroyl compound and extruding carbon monoxide. One of the first reported transition-metal mediated decarbonylations was reported from Eschinazi. ${ }^{77}$ Eschinazi found that aldehydes undergo decarbonylation in the presence of palladium. Later, Tsuji and Ohno reported the decarbonylation of primary or aryl aldehydes and acyl halides by stoichiometric amounts of Wilkinson's catalyst $\left(\mathrm{RhCl}\left(\mathrm{PPh}_{3}\right)_{3}\right)($ Scheme 24$) .{ }^{78}$ Since then, decarbonylation has been utilized in other functional groups including carboxylic acids ${ }^{79}$, esters ${ }^{80}$, anhydrides ${ }^{81}$, phthalimides ${ }^{82}$, and unstrained ketones. ${ }^{83}$

\section{Eschinazi}

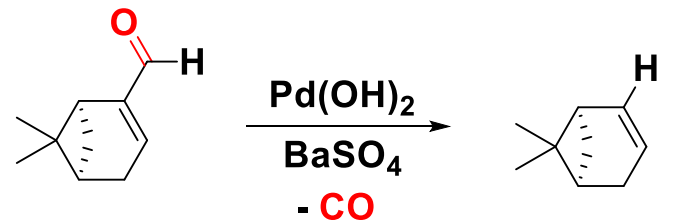

Tsuji and Ohno

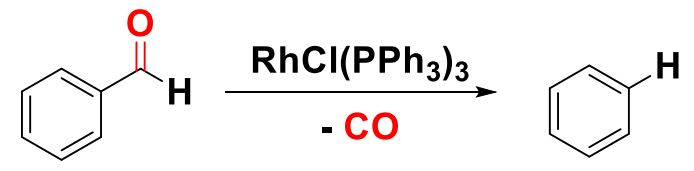

Scheme 24. First reported transition-metal-mediated decarbonylation.

More recently, nickel-catalyzed decarbonylative annulations of phthalimides have been reported. In 2008, Matsubara and Kurahashi postulated that a $\mathrm{Ni}(0)$ with electron-rich phosphines would oxidatively add to the $\mathrm{C}-\mathrm{N}$ bond thereby creating a $\mathrm{C}-\mathrm{Ni}-\mathrm{N}$ species 
after decarbonylation and allowing a variety of unsaturated alkynes to undergo insertion. ${ }^{84}$ There have also been reports of a directed nickel-catalyzed decarbonylation of unstrained ketones from $\mathrm{Xu}$ and $\mathrm{Wei}^{85}$ Where they found $\mathrm{N}$-pyrimidinyl 2-benzoyl indole as their model substrate would undergo decarbonylation to form a biaryl in the presence of $\mathrm{Ni}(\mathrm{COD})_{2}$ with a $\mathrm{NHC}$ and $\mathrm{Cs}_{2} \mathrm{CO}_{3}$ in dioxane. In addition, the directing group did not shut down the Ni-catalyst due to heterocyclic poisoning (Scheme 25).

\section{Matsubara and Kurahashi}<smiles>CN1C(=O)c2ccccc2C1=O</smiles>

Xu and Wei<smiles>O=C(c1ccccc1)c1cc2ccccc2n1-c1ncccn1</smiles>

$\mathrm{Ni}(\mathrm{COD})_{2}(10 \mathrm{~mol} \%)$

IMes• $\mathrm{HCl}(20 \mathrm{~mol} \%)$

$\mathrm{Cs}_{2} \mathrm{CO}_{3}$ (0.5 equiv)

Dioxane, $150^{\circ} \mathrm{C}$

$24 \mathrm{~h}$

$-\mathrm{CO}$

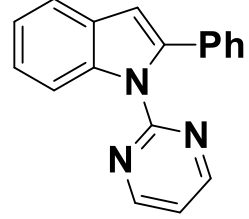

Scheme 25. Ni-catalyzed decarbonylation of unstrained ketones and phthalimides.

On the other hand, transition-metal catalyzed denitrogenative functionalization of triazole rings has attracted attention as an alternative to traditional synthesis for the construction of heterocyclic compounds. Early pioneering work from Gevorgyan ${ }^{86}$ and Fokin ${ }^{87}$ in Rh-catalyzed transannulation of triazoles with nitriles and alkynes highlighted this utility. Murakami and co-workers were the first to report a nickel-catalyzed denitrogenative annulation of 1,2,3-benzotriazin-4(3H)-ones with various unsaturated compounds. ${ }^{88}$ They discovered when using $\mathrm{Ni}(\mathrm{COD})_{2}$ in combination with a phosphine ligand, $\mathrm{Ni}(\mathrm{COD})_{2}$ inserted into the $\mathrm{N}-\mathrm{N}$ bond thereby producing dinitrogen and an azanickelacycle. This was later confirmed by adding stoichiometric amounts of $\mathrm{Ni}(\mathrm{COD})_{2}$ and Dppbenz to $\mathrm{N}$-toyl-1,2,3-benzotriazine-4(3H)-one to afford an isolable five-membered azanickelacycle (Scheme 26). ${ }^{89}$ Other groups have also reported annulation reactions with alkenes, ${ }^{90}$ isocyanides, ${ }^{91}$ benzynes, ${ }^{92}$ and cross-coupling with boronic acids. $^{93}$ 
<smiles>Cc1ccc(-n2nnc3ccccc3c2=O)cc1</smiles>
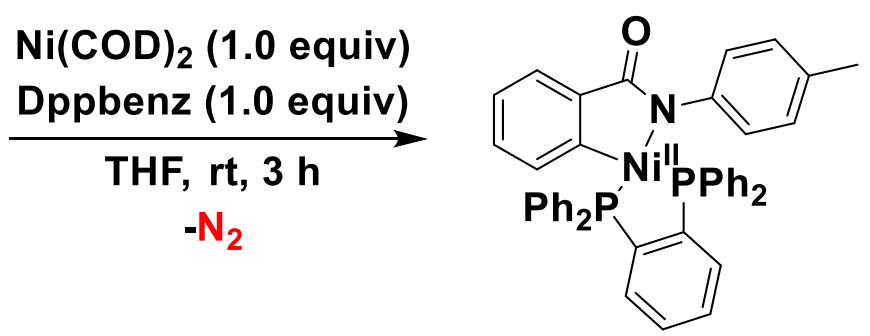

Scheme 26. Formation of a five-membered azanickelacycle by denitrogenation.

Since $\mathrm{Ni}(0)$ has been shown to insert in to the $\mathrm{C}-\mathrm{N}$ bond of phthalimide and the $\mathrm{N}-\mathrm{N}$ of triazole rings we hypothesized we would be able to synthesize the $\mathrm{Ni}$ (II) metallacycle from 3-(quinolin-8-yl)-3,4-dihydro-1,2,3-benzotriazin-4-one or 2-(quinolin-8-yl)-2,3dihydro-1H-isoindole-1,3-dione in the presence of a $\mathrm{Ni}(0)$ source (Scheme 27). Access to the targeted metallacycle would allow us to probe our proposed reaction mechanism, discussed earlier in Chapters 2 and 3.
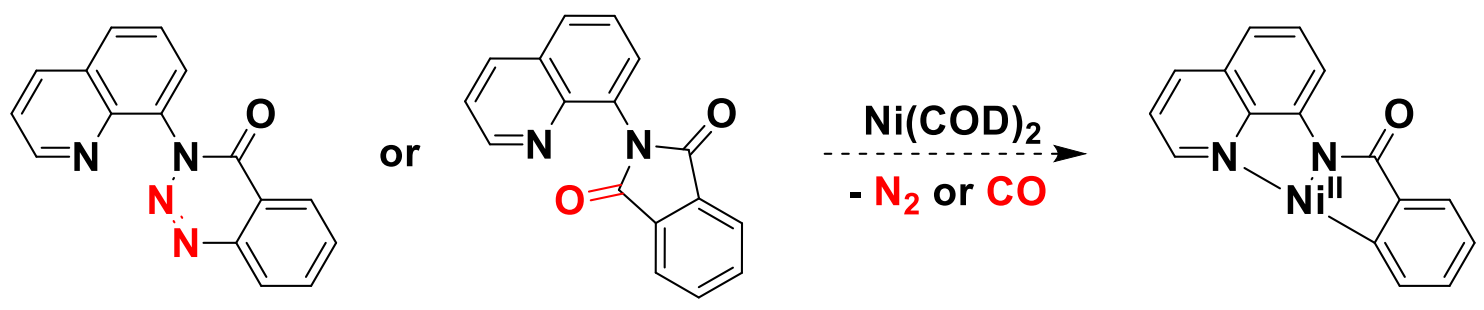

Scheme 27. Proposed synthesis of a Ni(II) metallacycle from phthalimide or 1,2,3benzotriazine.

\subsection{Results}

\subsubsection{Synthesis of 1,2,3-Benzotriazine Derivative and Attempted Denitrogenation for Ni(II) Metallacycle}

Since triazoles have been reported to undergo denitrogenation in the presence of $\mathrm{Ni}(\mathrm{COD})_{2}$, we set out to synthesize a 1,2,3-benzotriazine linked quinoline in hopes of isolating the proposed $\mathrm{Ni}(\mathrm{II})$ metallacycle. The 2-nitro- $N$-(quinolin-8-yl)benzamide was synthesized from a modified reported procedure. $^{94}$ 2-nitrobenzoyl chloride and 8aminoquinoline were allowed to stir overnight, providing $67 \%$ yield after purification. ${ }^{95}$ The corresponding 2-nitro- $N$-(quinolin-8-yl)benzamide was then reduced using a 
Béchamp reduction to afford the corresponding 2-amino- $N$-(quinolin-8-yl)benzamide. The substituted 2-aminobenazide product then afforded the desired annulation product from a reported procedure (Scheme 28). ${ }^{96}$
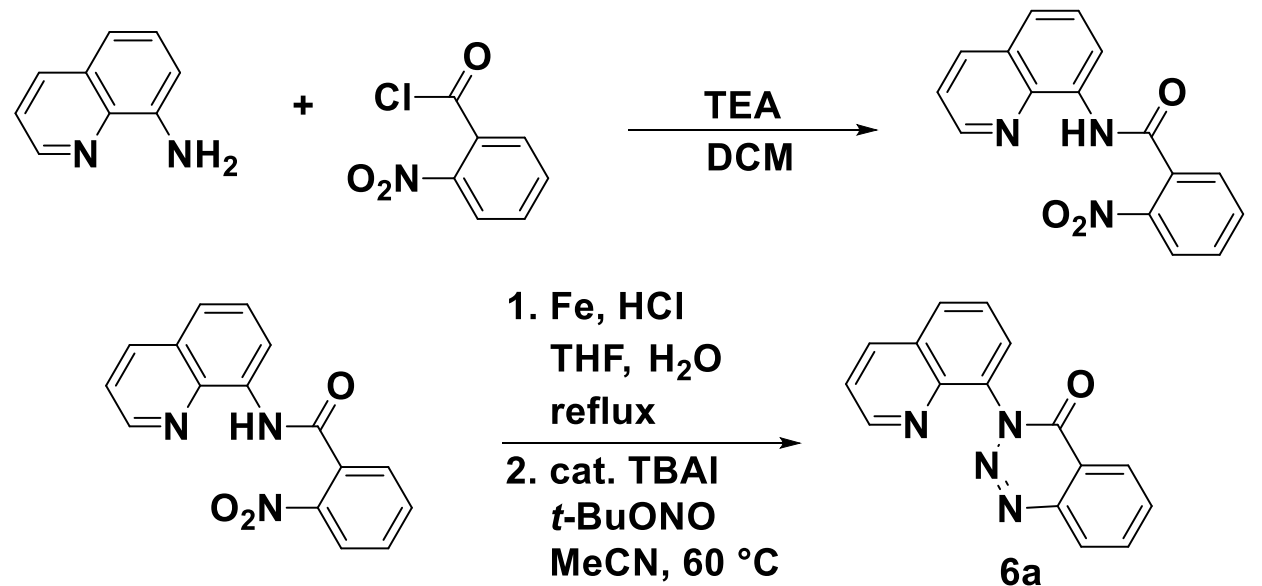

$6 a$

Scheme 28. Synthesis of 3-(quinolin-8-yl)-3,4-dihydro-1,2,3-benzotriazin-4-one from 8aminoquinoline and 2-nitrobenzoyl chloride.

The reaction of 3-(quinolin-8-yl)-3,4-dihydro-1,2,3-benzotriazin-4-one (6a) was treated with equimolar amounts of $\mathrm{Ni}(\mathrm{COD})_{2}$ and a ligand in $\mathrm{THF}$ at room temperature causing the solution to turn dark brown. After stirring for $3 \mathrm{~h}$, the mixture was concentrated down to minimum amount of THF. The reaction mixture was then precipitated out of solution into pentane dropwise producing a dark brown solid. The solid was then dried under high vacuum for $2 \mathrm{~h}$. 
Table 4.1. Attempted synthesis of a Ni(II) metallacycle by denitrogenation ${ }^{a}$

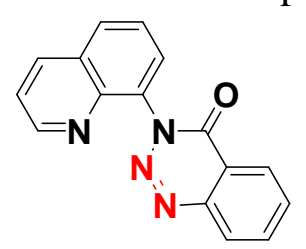

$6 a$

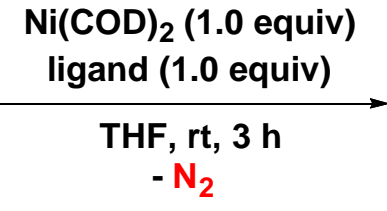

$-\mathrm{N}_{2}$

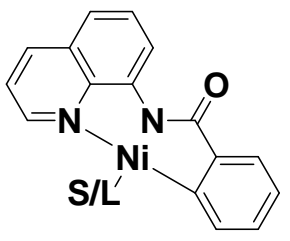

$7 a$

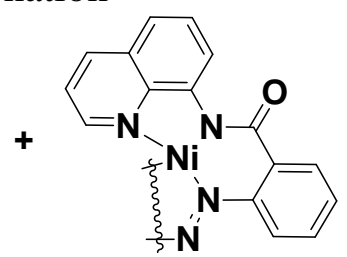

$8 a$

\begin{tabular}{llll}
\hline entry & Ligand & Yield 7a $(\%)$ & Yield 8a $(\%)$ \\
\hline 1 & $\mathrm{PPh}_{3}$ & 0 & 74 \\
2 & $\mathrm{MeCN}$ & 0 & 44 \\
3 & Dppbenz & 0 & 63 \\
4 & none & 0 & 71 \\
\hline
\end{tabular}

${ }^{a}$ Isolated yields. Reaction conditions: $6 \mathbf{a}(0.2 \mathrm{mmol}), \mathrm{Ni}(\mathrm{COD})_{2}(0.2 \mathrm{mmol})$, ligand $(0.2$ $\mathrm{mmol})$ in THF (8 $\mathrm{mL})$.

$\mathrm{Ni}(\mathrm{COD})_{2}$ was treated with $6 \mathbf{a}$ with in order to obtain the $\mathrm{Ni}(\mathrm{II})$ metallacycle complex. Characterization of the isolated products (Table 4.1, entry 1-4) showed the same diamagnetic ${ }^{1} \mathrm{H}$ NMR spectrum when ligands or no ligands were added. The ${ }^{1} \mathrm{H}$ NMR spectrum of 8a shows eight inequivalent aryl resonances adding up to ten protons. The ${ }^{13} \mathrm{C}\left\{{ }^{1} \mathrm{H}\right\}$ NMR spectrum shows fifteen different aryl resonances and one carbonyl resonance. The carbonyl resonance at $\delta 167.0$ is indicative of a typical six-membered azanickel metallacycle. ${ }^{97}$ Recrystallization of the reaction mixture from $\mathrm{CH}_{2} \mathrm{Cl}_{2} / \mathrm{Hexane}$ afforded complex 8a as dark brown crystals. The structure of 8a was established by single-crystal X-ray analysis to contain a square planar $\mathrm{Ni}(\mathrm{II})$ dimeric molecule in a bowl-shaped structure $\left[\left(\mathrm{C}_{16} \mathrm{H}_{10} \mathrm{~N}_{4} \mathrm{O}\right) \mathrm{Ni}\right]_{2}$ (Figure 2). 


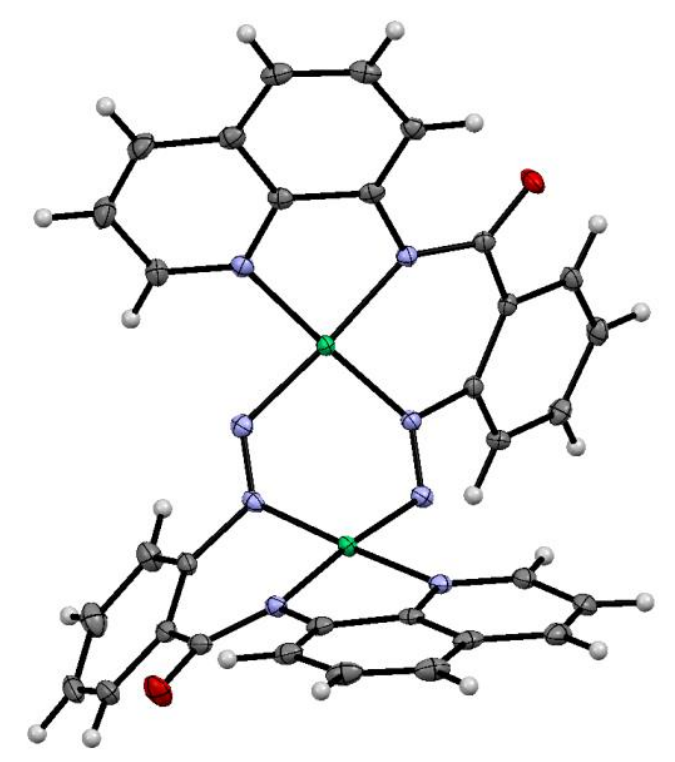

Figure 2. Perspective view of the molecular structure of $\left[\left(\mathrm{C}_{16} \mathrm{H}_{10} \mathrm{~N}_{4} \mathrm{O}\right) \mathrm{Ni}\right]_{2}(\mathbf{8 a})$ with the atom labeling scheme for the non-hydrogen atoms. The thermal ellipsoids are scaled to enclose $50 \%$ probability.

Intrigued by the formation of the six-membered azanickelacycle 8a, we explored the reactivity of the complex with our first reported ODC reaction conditions (Scheme 29). When $20 \mathrm{~mol} \%$ of complex 8a was substituted in place of $\mathrm{Ni}(\mathrm{OAc})_{2} \bullet 4 \mathrm{H}_{2} \mathrm{O}$, we discovered the ODC product $\mathbf{3 z}$ and $\mathbf{3 z}$ ' were formed with almost identical yields from our reported work. Likewise, when a stoichiometric amount of 8a was used, we noticed the diarylated ODC product 3z' was formed in higher yields than $\mathbf{3 z}$. This could be due to slow protonation of the $\mathrm{Ni}-\mathrm{N}$ bond allowing a second $\mathrm{C}-\mathrm{H}$ activation to take place. Surprised by these data, complex 8a could be undergoing a denitrogenation forming a $\mathrm{Ni}-\mathrm{C}$ bond. 
Scheme 29. Reactivity of complex 8a under the nickel-catalyzed oxidative decarboxylative (hetero)arylation reaction conditions.

(a) catalytic ${ }^{\text {a,c }}$
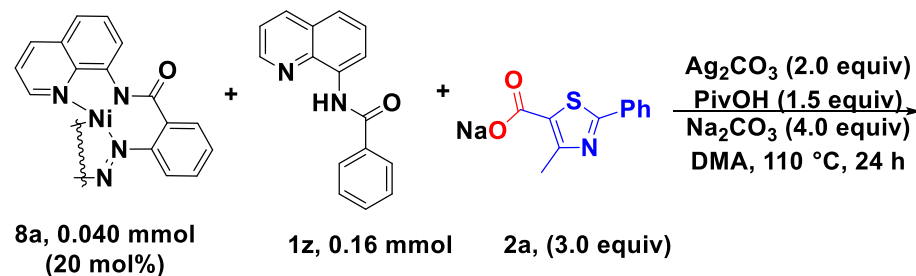

$12,0.16 \mathrm{mmol} \quad 2 \mathrm{a},(3.0$ equiv)

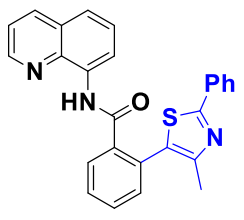

$3 z, 45 \%$

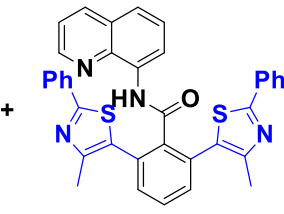

$3 z^{\prime}, 28 \%$

(b) stoichiometric ${ }^{b, c}$

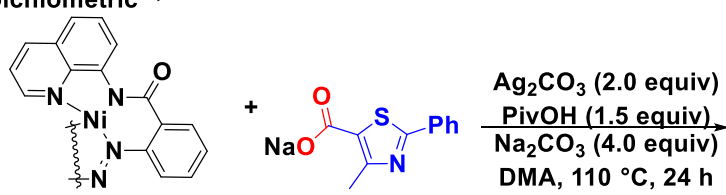

$8 \mathrm{a}, 0.20 \mathrm{mmol}$

2a, (3.0 equiv)

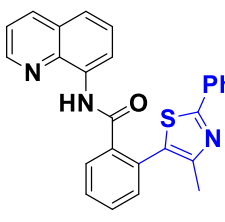

$3 z, 1 \%$

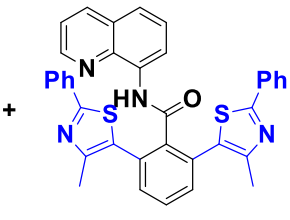

$3 z^{\prime}, 51 \%$

Reaction conditions: ${ }^{a}$ Azanickelacycle $8 \mathbf{a}(0.040 \mathrm{mmol}), \mathbf{1 a}(0.16 \mathrm{mmol})$, heteroaromatic carboxylate $2 \mathbf{a}(0.60 \mathrm{mmol}), \mathrm{Ag}_{2} \mathrm{CO}_{3}$ (2.0 equiv), $\mathrm{Na}_{2} \mathrm{CO}_{3}$ (4.0 equiv), and PivOH (1.5 equiv) in DMA ( $2 \mathrm{~mL}$ ) for $24 \mathrm{~h}$ at $110{ }^{\circ} \mathrm{C}$ under a $\mathrm{N}_{2}$ atmosphere. ${ }^{b}$ Azanickelacycle 8a (0.20 mmol), Heteroaromatic carboxylate $2 \mathbf{a}(0.60 \mathrm{mmol}), \mathrm{Ag}_{2} \mathrm{CO}_{3}$ (2.0 equiv), $\mathrm{Na}_{2} \mathrm{CO}_{3}$ (4.0 equiv), and PivOH (1.5 equiv) in DMA $(2 \mathrm{~mL})$ for $24 \mathrm{~h}$ at $110{ }^{\circ} \mathrm{C}$ under a $\mathrm{N}_{2}$ atmosphere. ${ }^{c 1} \mathrm{H}$ NMR yield with 1,3,5-trimethoxybenzene as an internal standard.

We hypothesized heating complex 8a in different solvents would force 8a to undergo denitrogenation, thereby creating the five-membered azanickelacycle. Heating of complex 8a in DCM- $d_{2}, \mathrm{MeCN}-d_{3}, \mathrm{THF}-d_{8}$, or DMSO- $d_{6}$ showed no change in the starting material by ${ }^{1} \mathrm{H}$ NMR spectroscopy. Heating of 8a in anhydrous DMA at $140{ }^{\circ} \mathrm{C}$ for $1 \mathrm{~h}$ led to a complex mixture of products. Upon switching the solvent to anhydrous DMF- $d_{7}$, we were able to see complex 8a was converted to a new species. Heating of the six-membered azanickelacycle 8a (red box outline), at $140{ }^{\circ} \mathrm{C}$ for $1 \mathrm{~h}$ led to formation of a new unknown product (Figure 3). Heating of the reaction over time and increasing temperature led to the formation of new unknown products. However, over time, there was a decrease in yield of the new product from $62 \%$ to $37 \%$. Attempts to isolate the unknown product after $1 \mathrm{~h}$ were unsuccessful. 


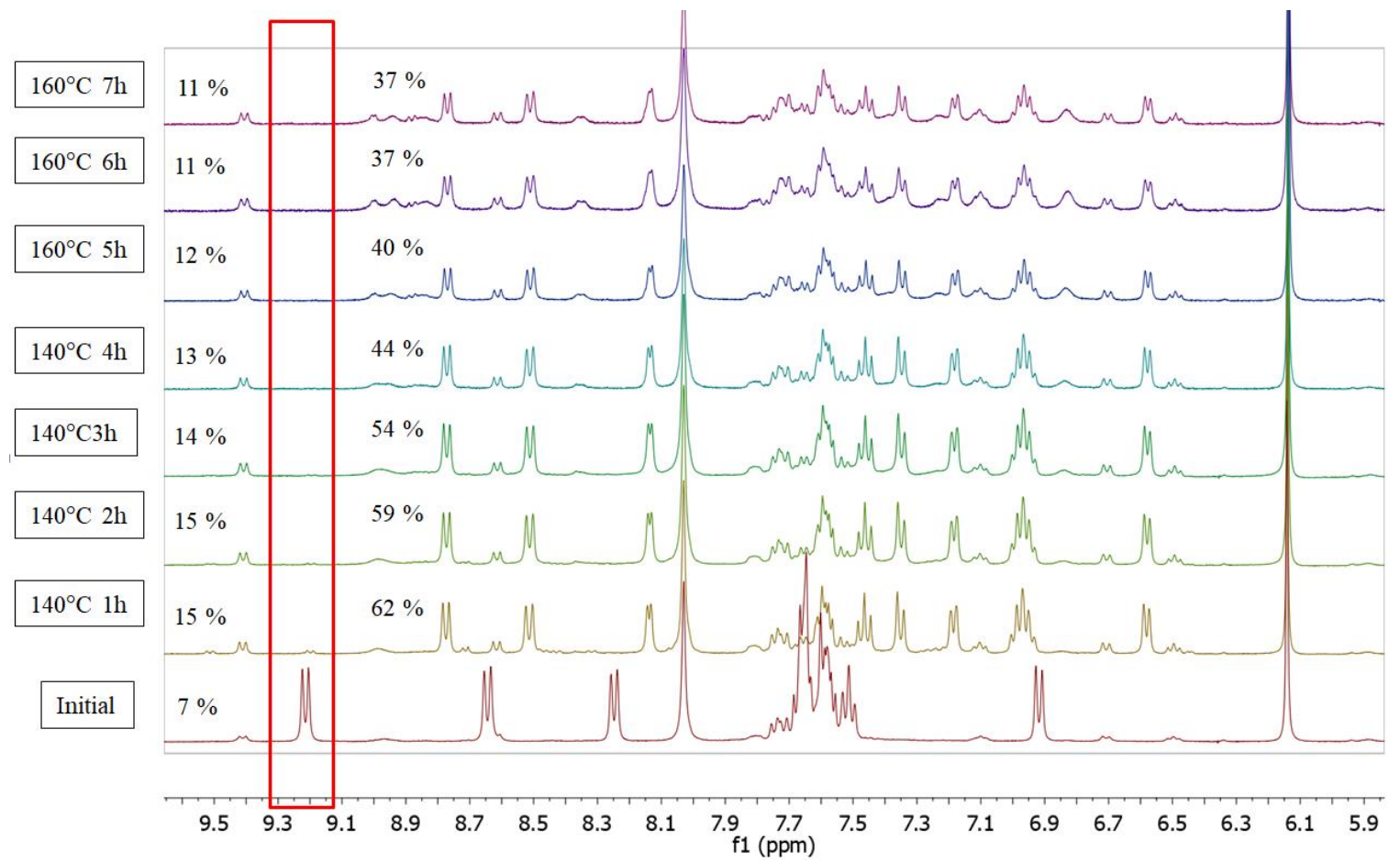

Figure 3. Heating of 8a in DMF- $d_{7}$ : Reaction conditions: 8a $(0.015 \mathrm{mmol})$ in DMF- $d_{7}$ $(0.90 \mathrm{~mL})$ with $1,3,5$-trimethoxybenzene as an internal standard at $140{ }^{\circ} \mathrm{C}$ to $160{ }^{\circ} \mathrm{C}$.

\subsubsection{Synthesis of the Ni(II) Metallacycle by Decarbonylation of Phthalimides}

To obtain the desired five-membered azanickelacycle, we then tried a different synthetic route, decarbonylation of phthalimides. The 2-(quinolin-8-yl)-2,3-dihydro-1Hisoindole-1,3-dione was synthesized from a reported procedure. ${ }^{98}$ Phthalic anhydride and 8 -aminoquinoline were refluxed in acetic acid for $2 \mathrm{~h}$, providing $79 \%$ yield of $9 \mathbf{a}$ following recrystallization (Scheme 30). The phthalimide was found to be soluble in THF, MeCN, and $\mathrm{PhCH}_{3}$ allowing for potential synthesis of the desired $\mathrm{Ni}(\mathrm{II})$ metallacycle. 

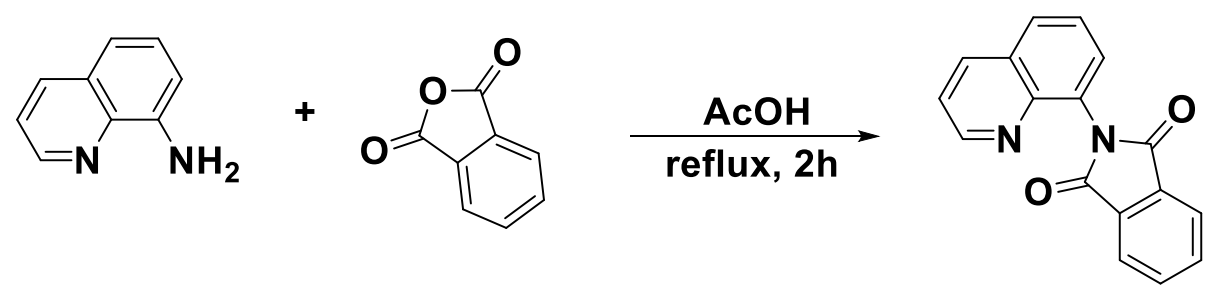

9a

Scheme 30. Synthesis of 2-(quinolin-8-yl)-2,3-dihydro-1H-isoindole-1,3-dione (9a) from 8-aminoquinoline and phthalic anhydride.

The reaction of 2-(quinolin-8-yl)-2,3-dihydro-1H-isoindole-1,3-dione (9a) was treated with equimolar amounts of $\mathrm{Ni}(\mathrm{COD})_{2}$ in $\mathrm{THF}$ at room temperature. Coordination of the phthalimide to nickel is signaled by a change in color in the solution from clear to dark red. After stirring for $3 \mathrm{~h}$, the mixture produced a light red precipitate that was filtered out of solution in (Scheme 31). The ${ }^{1} \mathrm{H}$ NMR spectrum of 10a shows eight inequivalent aryl resonances adding up to ten protons. The ${ }^{13} \mathrm{C}\left\{{ }^{1} \mathrm{H}\right\}$ NMR spectrum shows fifteen different aryl resonances and two carbonyl resonance. The carbonyl resonance at $\delta 167.23$ is indicative of a typical six-membered $\mathrm{Ni}$ (II) metallacycle while the second carbonyl resonance at $\delta 268.82$ is from insertion of $\mathrm{Ni}(\mathrm{COD})_{2}$ into the $(\mathrm{O}) \mathrm{C}-\mathrm{N}$ bond. ${ }^{99}$

Scheme 31. Synthesis of an azaacylNi(II) complex (10a) from insertion of nickel into the phthalimide $\mathrm{C}-\mathrm{N}$ bond. ${ }^{a}$

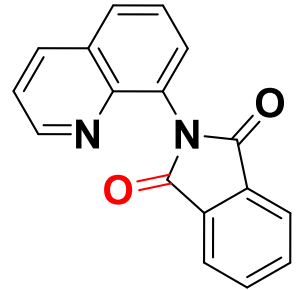

$9 a$

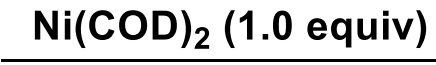
THF, rt, $3 \mathbf{h}$

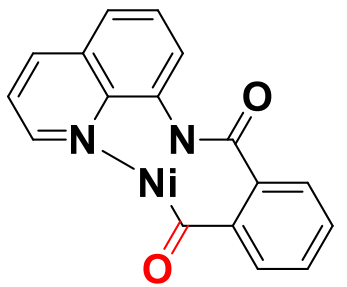

$10 a, 77 \%$

${ }^{a}$ Isolated yields. Reaction conditions: 9a $(1.0 \mathrm{mmol}), \mathrm{Ni}(\mathrm{COD})_{2}(1.0 \mathrm{mmol})$ in THF $(27$ $\mathrm{mL})$.

Complex 10a was tested under our nickel-catalyzed ODC reaction conditions to see if it had similar reactivity as complex 8a (Scheme 32). When 20 mol\% of complex 10a was substituted in place of $\mathrm{Ni}(\mathrm{OAc})_{2} \cdot 4 \mathrm{H}_{2} \mathrm{O}$, we found the ODC products $\mathbf{3 z}$ and $\mathbf{3 z}$ ' were formed in $37 \%$ and $21 \%$ yields. When a stoichiometric amount of complex 10a was used, there was a higher yield of the monoarylation ODC product $\mathbf{3 z}$ in $28 \%$ than the 
diarylation ODC product 3z' in $11 \%$ yield. While complex 10a shows reactivity under our reaction conditions, we were surprised to see differences in yields of $\mathbf{3 z}$ and $\mathbf{3 z}$ ' between complex 8a and 10a.

Scheme 32. Reactivity of azaacylNi(II) complex 10a under the nickel-catalyzed oxidative decarboxylative (hetero)arylation reaction conditions.

(a) catalytic ${ }^{\text {a,c }}$

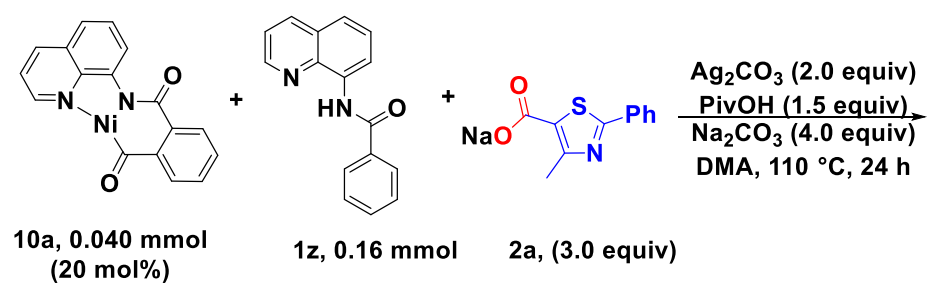

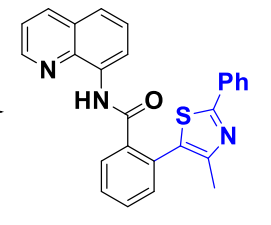

$3 z, 37 \%$

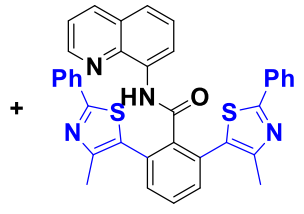

$3 z^{\prime}, 21 \%$

(b) stoichiometric ${ }^{b, c}$

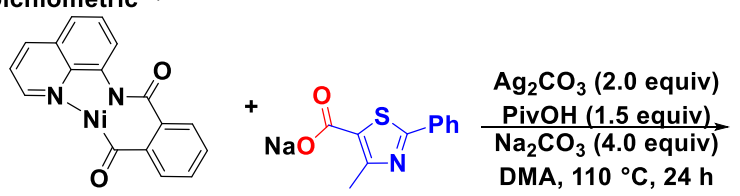

$10 \mathrm{a}, 0.20 \mathrm{mmol}$

$2 a,(3.0$ equiv)

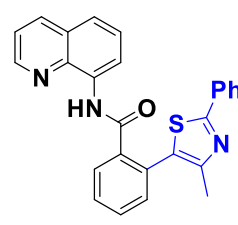

$3 z, 28 \%$

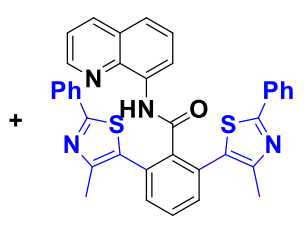

$3 z^{\prime}, 11 \%$

Reaction conditions: ${ }^{a}$ Azanickelacycle $\quad \mathbf{1 0 a} \quad(0.040 \quad \mathrm{mmol}), \quad \mathbf{1 a} \quad(0.16 \mathrm{mmol})$, heteroaromatic carboxylate $\mathbf{2 a}(0.60 \mathrm{mmol}), \mathrm{Ag}_{2} \mathrm{CO}_{3}$ (2.0 equiv), $\mathrm{Na}_{2} \mathrm{CO}_{3}$ (4.0 equiv), and PivOH (1.5 equiv) in DMA (2 mL) for $24 \mathrm{~h}$ at $110{ }^{\circ} \mathrm{C}$ under a $\mathrm{N}_{2}$ atmosphere. ${ }^{\mathrm{b}}$ Azanickelacycle 10a $(0.20 \mathrm{mmol})$, heteroaromatic carboxylate $\mathbf{2 a}(0.60 \mathrm{mmol}), \mathrm{Ag}_{2} \mathrm{CO}_{3}$ (2.0 equiv), $\mathrm{Na}_{2} \mathrm{CO}_{3}$ (4.0 equiv), and $\mathrm{PivOH}$ (1.5 equiv) in DMA ( $2 \mathrm{~mL}$ ) for $24 \mathrm{~h}$ at 110 ${ }^{\circ} \mathrm{C}$ under a $\mathrm{N}_{2}$ atmosphere. ${ }^{\mathrm{c} 1} \mathrm{H}$ NMR yield with 1,3,5-trimethoxybenzene as an internal standard.

With the azaacylNi(II) complex 10a in hand, we hypothesized the metallacycle would undergo decarbonylation at an elevated temperature. Heating complex 10a in $\mathrm{MeCN}$, dioxane, DMC, or THF led to decomposition with no formation of the desired product 11a. However, when complex 10a was heated in $\mathrm{PhCH}_{3}$, the solution turned from bright red to light yellow (see experimental below). Crystallization of the reaction mixture after heating afforded complex 11a as bright yellow parallelepiped crystals (Scheme 33). 


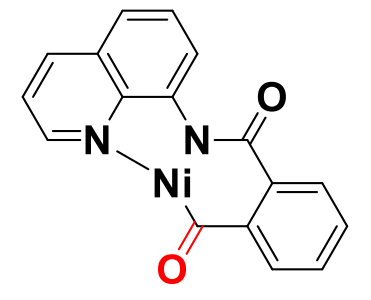

$10 a$

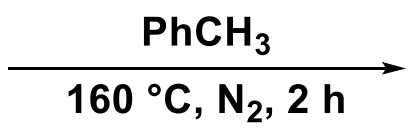

$160^{\circ} \mathrm{C}, \mathrm{N}_{2}, 2$

Scheme 33. Decarbonylation of the azaacylNi(II) metallacycle 10a to complex 11a.

The ${ }^{1} \mathrm{H}$ NMR spectrum of 11a shows nine inequivalent aryl resonances adding up to ten protons. The ${ }^{13} \mathrm{C}\left\{{ }^{1} \mathrm{H}\right\}$ NMR spectrum shows fifteen different aryl resonances and one carbonyl resonance. The carbonyl resonance at $\delta 186.96$ is indicative of a typical fivemembered azanickel metallacycle. ${ }^{89}$ The reaction mixture was allowed to cool to room temperature after heating affording complex 11a as bright yellow crystals. FTIR of complex 11a showed a characteristic carbonyl stretch at $2063 \mathrm{~cm}^{-1}$. The structure of 11a was established by single-crystal $\mathrm{X}$-ray analysis to contain a square planar $\mathrm{Ni}$ (II) molecule (Figure 4) 


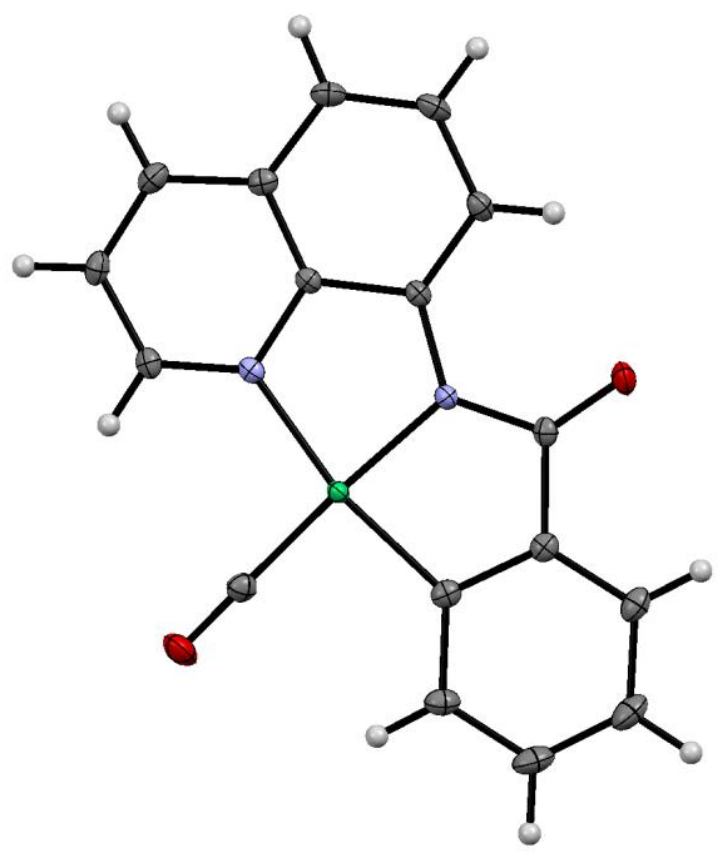

Figure 4. Perspective view of the molecular structure of $\left(\mathrm{C}_{16} \mathrm{H}_{10} \mathrm{~N}_{2} \mathrm{O}\right) \mathrm{Ni}(\mathrm{CO})$ (11a) with the atom labeling scheme for the non-hydrogen atoms. The thermal ellipsoids are scaled to enclose $50 \%$ probability.

After isolation of the new $\mathrm{Ni}(\mathrm{II})$ metallacycle, we wanted to probe our proposed catalytic cycle. We hypothesized in the previous chapters that silver is likely responsible for the decarboxylation step generating a well-defined silver aryl in solution, which then reactions with a nickel(II) metallacycle. To explore the possibility of such pathway, we conducted an experiment where complex 11a was treated with equimolar amounts of (2-nitrophenyl)silver(I) in DMA (Scheme 34). The initial reaction solution turned from orange/yellow to dark when the (2-nitrophenyl)silver(I) solution was added dropwise. The reaction was allowed to stir for $1 \mathrm{~h}$ at room temperature. After extraction of the reaction mixture, we were excited to see 13a was formed in $46 \%$ yield by ${ }^{1} \mathrm{H}$ NMR spectroscopy. When two equivalents of 12a were added, the yield of 13a increased to $68 \%$ (Scheme 34, entry 2). This transformation could be going through a transmetalation of the aryl fragment from $\mathrm{Ag}$ to $\mathrm{Ni}$ followed by concomitant oxidation of the nickel center to thereby generation the new $\mathrm{C}-\mathrm{C}$ bond. This data suggest there could be 
cooperation between both nickel and silver allowing for the remarkable efficiency and selectivity of our catalyst system as mentioned previously.

Scheme 34. Arylation of complex 11a with silver(I)-aryl for $\mathrm{C}-\mathrm{C}$ bond formation. ${ }^{a}$<smiles></smiles>

$11 \mathrm{a}$<smiles>O=[N+]([O-])c1ccccc1[Hg]</smiles>

$12 a$
DMA, r.t., $1 \mathrm{~h}$

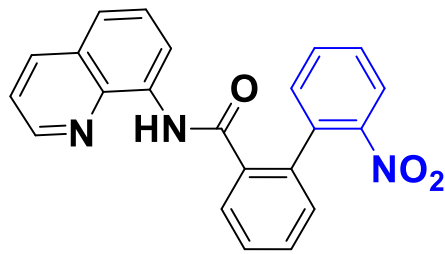

$13 a$

\begin{tabular}{ccc}
\hline entry & (12a) Equivalence & ${\text { yield }(\mathbf{1 3 a})^{\mathrm{b}}}$ \\
1 & 1.0 & $46 \%$ \\
2 & 2.0 & $68 \%$ \\
\hline
\end{tabular}

${ }^{a}$ Reaction conditions: 11a $(0.025 \mathrm{mmol}), \mathbf{1 2 a}(0.025 \mathrm{mmol}$ or $0.050 \mathrm{mmol})$, in DMA (2 $\mathrm{mL}$ ) for $1 \mathrm{~h}$ at room temperature under a $\mathrm{N}_{2}$ atmosphere. ${ }^{b 1} \mathrm{H}$ NMR yield with 1,3,5trimethoxybenzene as an internal standard.

\subsection{Conclusion}

In conclusion, we have synthesized a catalytically relevant five-membered azanickelacycle by decarbonylation of an azaacylNi(II) complex (10a). The attempted denitrogenation of 3-(quinolin-8-yl)-3,4-dihydro-1,2,3-benzotriazin-4-one (6a) afforded an interesting nickel metallacycle dimer, that showed reactivity under our earlier reported reaction conditions. Ultimately, the five-membered $\mathrm{Ni}$ (II) complex (11a) showed reactivity with (2-nitrophenyl)silver(I) to form the arylated product (13a). These findings support that both metals are needed to form the desired ODC product. 


\subsection{Experimental}

General Considerations. All manipulations were performed using standard Schlenk or glovebox techniques under a nitrogen atmosphere. All solvents (including dry DMA) were purchased from Alfa-Aesar and Fisher and used as received. All other reagents were purchased from Maybridge, Oakwood, Acros, Alfa-Aesar, and Strem and used without further purification. All NMR spectra were recorded at ambient temperature on an Agilent $400 \mathrm{MHz}$ or JEOL $400 \mathrm{MHz}\left({ }^{1} \mathrm{H}, 400 \mathrm{MHz} ;{ }^{13} \mathrm{C}\left\{{ }^{1} \mathrm{H}\right\}, 100 \mathrm{MHz}\right)$ spectrometer. Chemical shifts are referenced to the residual solvent signals $\left(\mathrm{CDCl}_{3}: 7.26 \mathrm{ppm}\left({ }^{1} \mathrm{H}\right)\right.$ and $77.2 \mathrm{ppm}\left({ }^{13} \mathrm{C}\right)$, DMSO- $d_{6}: 2.50 \mathrm{ppm}\left({ }^{1} \mathrm{H}\right)$ and $39.5 \mathrm{ppm}\left({ }^{13} \mathrm{C}\right), \mathrm{CD}_{2} \mathrm{Cl}_{2}: 5.32 \mathrm{ppm}\left({ }^{1} \mathrm{H}\right)$ and $\left.54.0 \mathrm{ppm}\left({ }^{13} \mathrm{C}\right)\right) .{ }^{45}$ IR spectra were recorded on a PerkinElmer (Spectrum 100) FT-IR spectrometer. Elemental analyses were performed by Atlantic Microlab, Inc., Norcross, GA. Column chromatography was performed using Silicycle Silica Flash P60 silica gel.

\section{Synthesis of 3-(quinolin-8-yl)-3,4-dihydro-1,2,3-benzotriazin-4-one (6a).}

Amide bond formation. A $100 \mathrm{~mL}$ two-neck round bottom flask was charged with 8aminoquinoline (1.44 g, $10.0 \mathrm{mmol}), \mathrm{Et}_{3} \mathrm{~N}(1.56 \mathrm{~mL}, 12.0 \mathrm{mmol})$, and methylene chloride (20 mL) under a $\mathrm{N}_{2}$ atmosphere. The corresponding 2-nitrobenzoyl chloride $(1.58 \mathrm{~mL}$, $12.0 \mathrm{mmol}$ ) was added dropwise at room temperature over 10 minutes. The mixture was stirred overnight at room temperature. The reaction was quenched with $10 \mathrm{~mL}$ of saturated $\mathrm{NaHCO}_{3}$ and extracted with methylene chloride $(3 \times 25 \mathrm{~mL})$. The combined organic layer was washed with brine $(15 \mathrm{~mL})$ and dried over anhydrous $\mathrm{Na}_{2} \mathrm{SO}_{4}$. The solvent was removed under reduced pressure and the residue was purified via silica gel column chromatography (gradient elution, hexanes : ethyl acetate $(5: 1, \mathrm{v} / \mathrm{v})$ to $(1: 1, \mathrm{v} / \mathrm{v}))$ yielding 2-nitro- $N$-(quinolin-8-yl)benzamide.

Reduction of 2-nitro- $\boldsymbol{N}$-(quinolin-8-yl)benzamide. A $50 \mathrm{~mL}$ round bottom flask was charged with water $(5 \mathrm{~mL})$, iron powder $(10.0 \mathrm{mmol}, 558 \mathrm{mg})$, and concentrated $\mathrm{HCl}(1.5$ drops) were added to a solution of 2-nitro- $N$-(quinolin-8-yl)benzamide (1.00 mmol, 293 $\mathrm{mg})$ in THF (15 mL). The mixture was refluxed for $3 \mathrm{~h}$. Upon completion, the reaction 
mixture was allowed to cool to room temperature, diluted with brine $(25 \mathrm{~mL})$ and filtered through a pad of celite. The filtrate was extracted with ethyl acetate $(3 \times 25 \mathrm{~mL})$ and dried over anhydrous $\mathrm{Na}_{2} \mathrm{SO}_{4}$. The solvent was removed under reduced pressure and the residue was purified via silica gel column chromatography (gradient elution, hexanes : ethyl acetate $(19: 1, \mathrm{v} / \mathrm{v})$ to $(10: 1, \mathrm{v} / \mathrm{v}))$ yielding 2-amino- $N$-(quinolin-8-yl)benzamide.

Annulation of 2-amino- $\boldsymbol{N}$-(quinolin-8-yl)benzamide. A oven dried Schlenk tube (50 $\mathrm{mL}$ ) was charged with 2-amino- $N$-(quinolin-8-yl)benzamide $(3.00 \mathrm{mmol}, 790 \mathrm{mg}$ ), TBAI (0.15 mmol, $55.4 \mathrm{mg})$, tert-butyl nitrite (9.00 mmol, $1.07 \mathrm{~mL})$, and $\mathrm{MeCN}$ (30 mL). The Schlenk tube was sealed with a rubber septum and placed in a pre-heated oil bath at 60 ${ }^{\circ} \mathrm{C}$ for $12 \mathrm{~h}$. The solution was cooled to room temperature and solvent was removed under reduced pressure. The residue was purified via silica gel column chromatography (gradient elution, hexanes : ethyl acetate $(1: 1, \mathrm{v} / \mathrm{v})$ to $(1: 2, \mathrm{v} / \mathrm{v}))$ yielding 3-(quinolin-8-yl)-3,4-dihydro-1,2,3-benzotriazin-4-one (6a) in 50\% (1.50 mmol, 417 $\mathrm{mg})$.

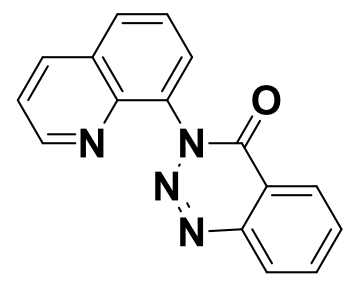

3-(quinolin-8-yl)-3,4-dihydro-1,2,3-benzotriazin-4-one (6a). ${ }^{1} \mathrm{H}$ NMR (400 $\mathrm{MHz}$, DMSO- $\left.d_{6}\right) \delta 8.85(\mathrm{dd}, J=4.2,1.7 \mathrm{~Hz}, 1 \mathrm{H}), 8.57(\mathrm{dd}, J=8.4,1.7 \mathrm{~Hz}, 1 \mathrm{H}), 8.35$ (dddd, $J$ $=8.0,4.7,1.4,0.6 \mathrm{~Hz}, 2 \mathrm{H}), 8.27(\mathrm{dd}, J=8.3,1.4 \mathrm{~Hz}, 1 \mathrm{H}), 8.20(\mathrm{ddd}, J=8.1,7.3,1.4 \mathrm{~Hz}$, $1 \mathrm{H}), 8.13(\mathrm{dd}, J=7.3,1.4 \mathrm{~Hz}, 1 \mathrm{H}), 8.03(\mathrm{ddd}, J=8.0,7.3,1.2 \mathrm{~Hz}, 1 \mathrm{H}), 7.86(\mathrm{dd}, J=8.3$, $7.3 \mathrm{~Hz}, 1 \mathrm{H}), 7.66(\mathrm{dd}, J=8.3,4.2 \mathrm{~Hz}, 1 \mathrm{H}) .{ }^{13} \mathrm{C}\left\{{ }^{1} \mathrm{H}\right\}$ NMR $\left(100 \mathrm{MHz}\right.$, DMSO- $\left.d_{6}\right) \delta$ $155.13,151.50,143.57,143.21,136.55,136.17,135.83,133.41,130.39$, 129.35, 128.64, $128.32,126.40,124.94,122.42,119.94$. The spectral data is consistent with literature. ${ }^{28}$ 


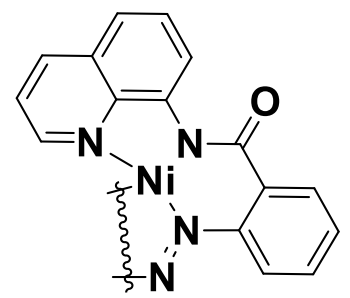

$\left[\left(\mathbf{C}_{16} \mathrm{H}_{10} \mathbf{N}_{4} \mathbf{O}\right) \mathbf{N i}\right]_{2} \quad(\mathbf{8 a})$. In a glovebox filled with $\mathrm{N}_{2}$. A mixture of 3-(quinolin-8-yl)-3,4-dihydro-1,2,3-benzotriazin-4-one ( $54.8 \mathrm{mg}, 0.200 \mathrm{mmol}$ ) was added to a $20 \mathrm{~mL}$ vial and dissolved in THF (4 mL). In another $20 \mathrm{~mL}$ vial, $\mathrm{Ni}(\mathrm{COD})_{2}(55.0 \mathrm{mg}$, $0.200 \mathrm{mmol})$ and ligand $(0.200 \mathrm{mmol})$ was dissolved in THF $(4 \mathrm{~mL})$. The solution of $\mathrm{Ni}(\mathrm{COD})_{2}$ was added dropwise to the vial containing 3-(quinolin-8-yl)-3,4-dihydro-1,2,3-benzotriazin-4-one and THF over 5 minutes. The solution was allowed to stir for $3 \mathrm{~h}$ at room temperature. The volume was then reduced to $\sim 1.5 \mathrm{~mL}$ and added dropwise to hexanes $(\sim 100 \mathrm{~mL})$ to precipitate out a dark brown solid. The solid was then dried under vacuum to give $47.2 \mathrm{mg}(0.142 \mathrm{mmol}, 71 \%) .{ }^{1} \mathrm{H}$ NMR $\left(400 \mathrm{MHz}, \mathrm{CD}_{2} \mathrm{Cl}_{2}\right) \delta 9.17(\mathrm{dd}, J=7.9,1.1 \mathrm{~Hz}, 2 \mathrm{H}), 8.30(\mathrm{dd}, J=8.3,1.5 \mathrm{~Hz}, 2 \mathrm{H}), 8.25$ $(\mathrm{dd}, J=7.9,1.5 \mathrm{~Hz}, 2 \mathrm{H}), 7.60(\mathrm{t}, J=8.0 \mathrm{~Hz}, 2 \mathrm{H}), 7.56-7.47(\mathrm{~m}, 4 \mathrm{H}), 7.44-7.33(\mathrm{~m}$, $4 \mathrm{H}), 7.27(\mathrm{dd}, J=8.2,5.1 \mathrm{~Hz}, 2 \mathrm{H}), 6.78(\mathrm{dd}, J=7.9,1.2 \mathrm{~Hz}, 2 \mathrm{H}) .{ }^{13} \mathrm{C}\left\{{ }^{1} \mathrm{H}\right\}$ NMR $(100$ $\left.\mathrm{MHz}, \mathrm{CD}_{2} \mathrm{Cl}_{2}\right) \delta 167.03,151.78,149.18,147.96,144.85,139.86,131.53,130.92,130.56$, $130.12,130.08,129.27,122.84,121.85,119.71,119.14$.

Heating of $\left[\left(\mathrm{C}_{16} \mathrm{H}_{10} \mathrm{~N}_{4} \mathrm{O}\right) \mathrm{Ni}\right]_{2}(8 \mathrm{a})$ in J. Young NMR tube. In a glovebox filled with $\mathrm{N}_{2}$. A J. Young NMR tube was charged with azanickelacycle 8a $(5.0 \mathrm{mg}, 0.015 \mathrm{mmol}$ ), 1,3,5-trimethoxybenzene $(1.3 \mathrm{mg}, 0.0077 \mathrm{mmol})$, and DMF- $d_{7}(0.90 \mathrm{~mL})$. The J. Young NMR tube was taken out of the glovebox and placed in a preheated oil bath at $140{ }^{\circ} \mathrm{C}$. The reaction was monitored by ${ }^{1} \mathrm{H}$ NMR every hour. After $4 \mathrm{~h}$, the oil bath was heated to $160{ }^{\circ} \mathrm{C}$ and monitored by ${ }^{1} \mathrm{H}$ NMR every hour. 


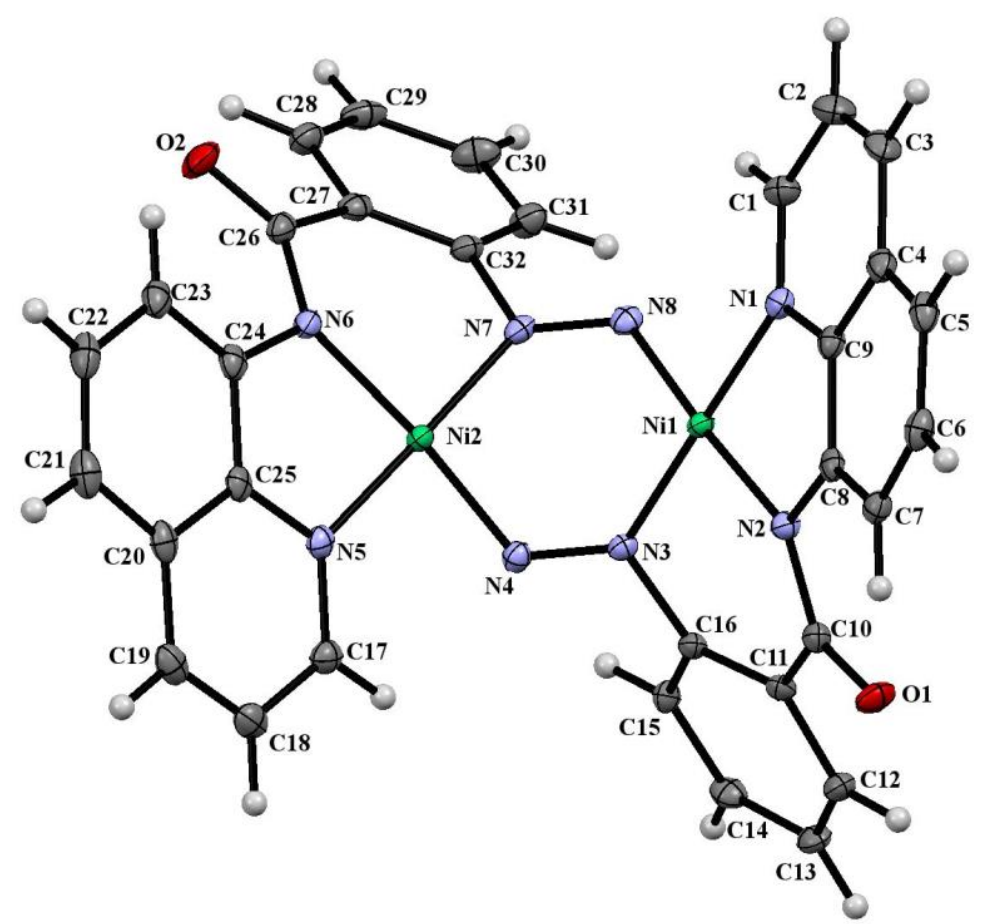

Perspective view of the molecular structure of $\left[\left(\mathrm{C}_{16} \mathrm{H}_{10} \mathrm{~N}_{4} \mathrm{O}\right) \mathrm{Ni}\right]_{2}(\mathbf{8 a})$ with the atom labeling scheme for the non-hydrogen atoms. The thermal ellipsoids are scaled to enclose $50 \%$ probability. The $\mathrm{Ni}(1) \cdots \mathrm{Ni}(2)$ interatomic distance is $3.285 \AA$. The acute dihedral angle between the plane containing atoms $\mathrm{Ni}(1), \mathrm{N}(1), \mathrm{N}(2), \mathrm{N} 3), \mathrm{N}(8)$ and the plane containing atoms $\mathrm{Ni}(2), \mathrm{N}(4), \mathrm{N} 5), \mathrm{N}(6), \mathrm{N}(7)$ is $77.2^{\circ}$.

\section{Description of the X-ray Structural Analysis of $\left[\left(\mathrm{C}_{16} \mathrm{H}_{10} \mathrm{~N}_{4} \mathrm{O}\right) \mathrm{Ni}_{2} \cdot \mathrm{CH}_{2} \mathrm{Cl}_{2}(8 \mathrm{a})\right.$}

A dark brown parallelepiped crystal of $\left[\left(\mathrm{C}_{16} \mathrm{H}_{10} \mathrm{~N}_{4} \mathrm{O}\right) \mathrm{Ni}_{2} \cdot \mathrm{CH}_{2} \mathrm{Cl}_{2}(\mathbf{8 a})\right.$ was coated in polybutene oil (Sigma-Aldrich) and placed on the end of a MiTeGen loop. The sample was cooled to $100 \mathrm{~K}$ with an Oxford Cryostream 700 system and optically aligned on a Bruker AXS D8 Venture fixed-chi X-ray diffractometer equipped with a Triumph monochromator, a Mo K $\alpha$ radiation source $(\lambda=0.71073 \AA$ ), and a PHOTON 100 CMOS detector. Three sets of 12 frames each were collected using the omega scan method with a 10 second exposure time. Integration of these frames followed by reflection indexing and least-squares refinement produced a crystal orientation matrix for the monoclinic crystal lattice that was used for the structural analysis. 
Data collection consisted of the measurement of a total of 1056 frames in four runs using omega scans with the detector held at $5.00 \mathrm{~cm}$ from the crystal. Frame scan parameters are summarized below:

Data collection details for $\left[\left(\mathrm{C}_{16} \mathrm{H}_{10} \mathrm{~N}_{4} \mathrm{O}\right) \mathrm{Ni}\right]_{2} \cdot \mathrm{CH}_{2} \mathrm{Cl}_{2}(8 \mathrm{a})$.

\begin{tabular}{|c|c|c|c|c|c|c|c|}
\hline Run & $2 \theta$ & $\omega$ & $\varphi$ & $\chi$ & Scan Width $\left(^{\circ}\right)$ & Frames & $\begin{array}{c}\text { Exposure } \\
\text { Time (sec) }\end{array}$ \\
\hline 1 & 11.01 & -171.48 & 144.00 & 54.74 & 0.70 & 264 & 60.00 \\
\hline 2 & 11.01 & -171.48 & 72.00 & 54.74 & 0.70 & 264 & 60.00 \\
\hline 3 & 11.01 & -171.48 & -144.00 & 54.74 & 0.70 & 264 & 60.00 \\
\hline 4 & 11.01 & -171.48 & 0.00 & 54.74 & 0.70 & 264 & 60.00 \\
\hline
\end{tabular}

The APEX3 software program (version 2016.9-0) ${ }^{100}$ was used for diffractometer control, preliminary frame scans, indexing, orientation matrix calculations, least-squares refinement of cell parameters, and the data collection. The frames were integrated with the Bruker SAINT software package using a narrow-frame algorithm. The integration of the data using a monoclinic unit cell yielded a total of 58425 reflections to a maximum $\theta$ angle of $27.98^{\circ}(0.76 \AA$ resolution), of which 7038 were independent (average redundancy 8.301, completeness $\left.=98.4 \%, \mathrm{R}_{\text {int }}=6.17 \%, \mathrm{R}_{\mathrm{sig}}=4.35 \%\right)$ and 5358 (76.13\%) were greater than $2 \sigma\left(F^{2}\right)$. The final cell constants of $\underline{a}=12.1353(10) \AA, \underline{b}=$ 24.815(2) $\AA, \underline{c}=10.1263(9) \AA, \beta=103.132(2)^{\circ}$, volume $=2969.7(4) \AA^{3}$, are based upon the refinement of the XYZ-centroids of 9287 reflections above $20 \sigma(\mathrm{I})$ with $6.174^{\circ}<2 \theta<$ $54.87^{\circ}$. Data were corrected for absorption effects using the multi-scan method (SADABS). The ratio of minimum to maximum apparent transmission was 0.850 . The calculated minimum and maximum transmission coefficients (based on crystal size) are 0.437 and 0.922 .

The structure was solved by direct methods and difference Fourier analyses using the programs provided by SHELXL-2014/7. ${ }^{101}$ Idealized positions for the methylene and aromatic hydrogen atoms were included as fixed contributions using a riding model with isotropic temperature factors set at 1.2 times that of the adjacent carbon atom. Full-matrix least-squares refinement, based upon the minimization of $\Sigma \mathrm{w}_{\mathrm{i}}\left|\mathrm{F}_{\mathrm{o}}{ }^{2}-\mathrm{F}_{\mathrm{c}}{ }^{2}\right|^{2}$, with weighting 
$\mathrm{W}_{\mathrm{i}}^{-1}=\left[\sigma^{2}\left(\mathrm{~F}_{\mathrm{o}}{ }^{2}\right)+(0.0203 \mathrm{P})^{2}+2.9372 \mathrm{P}\right]$, where $\mathrm{P}=\left(\operatorname{Max}\left(\mathrm{F}_{\mathrm{o}}{ }^{2}, 0\right)+2 \mathrm{~F}_{\mathrm{c}}{ }^{2}\right) / 3 .^{2}$ The final anisotropic full-matrix least-squares refinement on $\mathrm{F}^{2}$ with 424 variables converged at $\mathrm{R} 1$ $=3.06 \%$ for the 5358 data with $\mathrm{I}>2 \sigma(\mathrm{I})$ and $\mathrm{wR} 2=5.58 \%$ for all data. The goodness-offit was $1.016 .^{102}$

A correction for secondary extinction was not applied. The largest peak in the final difference electron density synthesis was $0.399 \mathrm{e}^{-} / \AA^{3}$ and the largest hole was $-0.545 \mathrm{e}^{-}$ $/ \AA^{3}$ with an RMS deviation of $0.079 \mathrm{e}^{-} / \AA^{3}$. The linear absorption coefficient, atomic scattering factors, and anomalous dispersion corrections were calculated from values found in the International Tables of X-ray Crystallography. ${ }^{103}$

\section{Crystal data for $\left[\left(\mathrm{C}_{16} \mathrm{H}_{10} \mathrm{~N}_{4} \mathrm{O}\right) \mathrm{Ni}\right]_{2} \cdot \mathrm{CH}_{2} \mathrm{Cl}_{2}(8 \mathrm{a})$.}

Ident. code jh53 cms

Chem. form. $\mathrm{C}_{33} \mathrm{H}_{22} \mathrm{Cl}_{2} \mathrm{~N}_{8} \mathrm{Ni}_{2} \mathrm{O}_{2}$

Mol. weight $750.90 \mathrm{~g} / \mathrm{mol}$

Temperature 100(2) K

Wavelength $0.71073 \AA$

Crystal size $0.055 \times 0.111 \times 0.663 \mathrm{~mm}$

Cryst. type monoclinic

Space group $\mathrm{P} 2{ }_{1} / \mathrm{c}$ (No. 14)

$$
\begin{array}{rll}
\text { Unit cell } & \mathrm{a}=12.1353(10) \AA & \alpha=90^{\circ} \\
\mathrm{b}=24.815(2) \AA & \beta=103.132(2)^{\circ} \\
\mathrm{c}=10.1263(9) \AA & \gamma=90^{\circ}
\end{array}
$$

Volume, $\AA^{3}$ 2969.7(4)

Z 4

Density $\quad 1.679 \mathrm{~g} / \mathrm{cm}^{3}$

(calc)

Abs. coeff. $1.497 \mathrm{~mm}^{-1}$

$\mathrm{F}(000) \quad 1528$ 
Data collection and structure refinement for $\left[\left(\mathrm{C}_{16} \mathrm{H}_{10} \mathrm{~N}_{4} \mathrm{O}\right) \mathrm{Ni}_{2}\right]_{2} \mathrm{CH}_{2} \mathrm{Cl}_{2}(\mathbf{8 a})$.

$\begin{array}{ll}\text { Theta range } & 2.88 \text { to } 27.98^{\circ} \\ \text { Index ranges } & -15 \leq \mathrm{h} \leq 15,-32 \leq \mathrm{k} \leq 32,-13 \leq 1 \leq 13 \\ \text { Reflections } & 58425 \\ \text { Independent refls } & 7038[\mathrm{R}(\mathrm{int})=0.0617] \\ \text { Coverage } & 98.4 \%\end{array}$

Abs. correction multi-scan

Max./ min. trans. $\quad 0.922$ and 0.437

Refinement method Full-matrix least-squares on $\mathrm{F}^{2}$

Ref. program SHELXL-2014/7 (Sheldrick, 2014)

Data / restrs /

$7038 / 0 / 424$

parms

GOF on $\mathrm{F}^{2} \quad 1.016$

$\begin{array}{ll}5358 \text { data; } & \mathrm{R} 1=0.0306, \mathrm{wR} 2=0.0587\end{array}$

$$
\text { all data } \quad \mathrm{R} 1=0.0558, \mathrm{wR} 2=0.0655
$$

Largest diff. peak and hole

$$
0.399 \text { and }-0.545 \mathrm{e}^{-} / \AA^{3}
$$




\begin{tabular}{|c|c|c|c|c|}
\hline & $\mathrm{x} / \mathrm{a}$ & $\mathrm{y} / \mathrm{b}$ & $\mathrm{z} / \mathrm{c}$ & $\mathrm{U}(\mathrm{eq})$ \\
\hline $\mathrm{O} 2$ & $0.05756(13)$ & $0.04119(6)$ & $0.24573(16)$ & $0.0232(4)$ \\
\hline N1 & $0.59986(14)$ & $0.15761(7)$ & $0.36212(17)$ & $0.0134(4)$ \\
\hline $\mathrm{N} 2$ & $0.56869(14)$ & $0.25817(6)$ & $0.30274(17)$ & $0.0120(4)$ \\
\hline $\mathrm{N} 3$ & $0.37741(14)$ & $0.25818(6)$ & $0.40015(17)$ & $0.0113(3)$ \\
\hline N4 & $0.27252(14)$ & $0.25286(7)$ & $0.36729(17)$ & $0.0128(4)$ \\
\hline N5 & $0.11739(14)$ & $0.22296(7)$ & $0.14396(17)$ & $0.0133(4)$ \\
\hline N6 & $0.13645(14)$ & $0.12434(7)$ & $0.22252(18)$ & $0.0130(4)$ \\
\hline N7 & $0.31233(14)$ & $0.15094(6)$ & $0.43374(17)$ & $0.0121(4)$ \\
\hline N8 & $0.41704(14)$ & $0.15655(7)$ & $0.46962(17)$ & $0.0133(4)$ \\
\hline $\mathrm{C} 1$ & $0.61749(18)$ & $0.10772(8)$ & $0.4094(2)$ & $0.0173(5)$ \\
\hline $\mathrm{C} 2$ & $0.69670(19)$ & $0.07351(9)$ & $0.3707(2)$ & $0.0227(5)$ \\
\hline $\mathrm{C} 3$ & $0.75627(19)$ & $0.09119(9)$ & $0.2793(2)$ & $0.0203(5)$ \\
\hline $\mathrm{C} 4$ & $0.73955(18)$ & $0.14411(8)$ & $0.2266(2)$ & $0.0161(4)$ \\
\hline C5 & $0.79267(18)$ & $0.16575(9)$ & $0.1272(2)$ & $0.0172(5)$ \\
\hline C6 & $0.77024(18)$ & $0.21788(9)$ & $0.0860(2)$ & $0.0175(5)$ \\
\hline $\mathrm{C} 7$ & $0.69883(17)$ & $0.25184(8)$ & $0.1413(2)$ & $0.0142(4)$ \\
\hline $\mathrm{C} 8$ & $0.64308(17)$ & $0.23187(8)$ & $0.2360(2)$ & $0.0126(4)$ \\
\hline C9 & $0.66175(17)$ & $0.17688(8)$ & $0.2744(2)$ & $0.0132(4)$ \\
\hline $\mathrm{C} 10$ & $0.57337(17)$ & $0.31253(8)$ & $0.3178(2)$ & $0.0130(4)$ \\
\hline $\mathrm{C} 11$ & $0.50696(17)$ & $0.33581(8)$ & $0.4138(2)$ & $0.0121(4)$ \\
\hline $\mathrm{C} 12$ & $0.53571(18)$ & $0.38774(8)$ & $0.4635(2)$ & $0.0152(4)$ \\
\hline $\mathrm{C} 13$ & $0.48073(18)$ & $0.41252(8)$ & $0.5530(2)$ & $0.0162(4)$ \\
\hline C14 & $0.39442(18)$ & $0.38582(8)$ & $0.5957(2)$ & $0.0163(4)$ \\
\hline $\mathrm{C} 15$ & $0.36168(17)$ & $0.33496(8)$ & $0.5447(2)$ & $0.0137(4)$ \\
\hline $\mathrm{C} 16$ & $0.41704(17)$ & $0.31082(8)$ & $0.4546(2)$ & $0.0120(4)$ \\
\hline
\end{tabular}




\begin{tabular}{rlrrr} 
& \multicolumn{1}{c}{ x/a } & y/b & z/c & U(eq) \\
C17 & $0.10322(18)$ & $0.27529(8)$ & $0.1189(2)$ & $0.0155(4)$ \\
C18 & $0.03540(18)$ & $0.29448(9)$ & $0.9969(2)$ & $0.0180(5)$ \\
C19 & $0.98563(18)$ & $0.25885(9)$ & $0.8980(2)$ & $0.0186(5)$ \\
C20 & $0.00015(17)$ & $0.20288(9)$ & $0.9207(2)$ & $0.0164(4)$ \\
C21 & $0.95287(18)$ & $0.16230(9)$ & $0.8261(2)$ & $0.0206(5)$ \\
C22 & $0.97150(18)$ & $0.10920(10)$ & $0.8610(2)$ & $0.0213(5)$ \\
C23 & $0.03199(17)$ & $0.09301(9)$ & $0.9907(2)$ & $0.0183(5)$ \\
C24 & $0.07803(17)$ & $0.13135(8)$ & $0.0865(2)$ & $0.0149(4)$ \\
C25 & $0.06506(16)$ & $0.18645(8)$ & $0.0480(2)$ & $0.0131(4)$ \\
C26 & $0.11679(17)$ & $0.08050(8)$ & $0.2926(2)$ & $0.0154(4)$ \\
C27 & $0.16762(17)$ & $0.08165(8)$ & $0.4435(2)$ & $0.0150(4)$ \\
C28 & $0.11652(19)$ & $0.04896(8)$ & $0.5241(2)$ & $0.0191(5)$ \\
C29 & $0.15311(19)$ & $0.04724(9)$ & $0.6637(2)$ & $0.0211(5)$ \\
C30 & $0.2482(2)$ & $0.07672(9)$ & $0.7260(2)$ & $0.0230(5)$ \\
C31 & $0.30268(19)$ & $0.10861(9)$ & $0.6482(2)$ & $0.0195(5)$ \\
C32 & $0.26091(17)$ & $0.11193(8)$ & $0.5090(2)$ & $0.0136(4)$ \\
C33 & $0.7187(2)$ & $0.46528(9)$ & $0.2612(2)$ & $0.0245(5)$ \\
C11 & $0.59581(6)$ & $0.49793(3)$ & $0.16820(7)$ & $0.03478(16)$ \\
C12 & $0.75204(5)$ & $0.48583(2)$ & $0.43372(6)$ & $0.02809(14)$
\end{tabular}

Interatomic distances $(\AA)$ for $\left[\left(\mathrm{C}_{16} \mathrm{H}_{10} \mathrm{~N}_{4} \mathrm{O}\right) \mathrm{Ni}\right]_{2} \cdot \mathrm{CH}_{2} \mathrm{Cl}_{2}$ (8a).

$\begin{array}{llll}\text { Ni1-N8 } & 1.8347(17) & \text { Ni1-N3 } & 1.8531(17) \\ \text { Ni1-N1 } & 1.9086(17) & \text { Ni1-N2 } & 1.9109(17) \\ \text { Ni2-N4 } & 1.8301(17) & \text { Ni2-N7 } & 1.8624(17) \\ \text { Ni2-N6 } & 1.9085(16) & \text { Ni2-N5 } & 1.9179(17)\end{array}$




\begin{tabular}{|c|c|c|c|}
\hline O1-C10 & $1.233(2)$ & $\mathrm{O} 2-\mathrm{C} 26$ & $1.240(2)$ \\
\hline $\mathrm{N} 1-\mathrm{C} 1$ & $1.327(3)$ & N1-C9 & $1.373(3)$ \\
\hline $\mathrm{N} 2-\mathrm{C} 10$ & $1.357(3)$ & N2-C8 & $1.405(3)$ \\
\hline N3-N4 & $1.247(2)$ & N3-C16 & $1.456(2)$ \\
\hline N5-C17 & $1.327(3)$ & N5-C25 & $1.374(3)$ \\
\hline N6-C26 & $1.349(3)$ & N6-C24 & $1.409(3)$ \\
\hline N7-N8 & $1.247(2)$ & N7-C32 & $1.457(3)$ \\
\hline $\mathrm{C} 1-\mathrm{C} 2$ & $1.404(3)$ & $\mathrm{C} 2-\mathrm{C} 3$ & $1.370(3)$ \\
\hline C3-C4 & $1.415(3)$ & C4-C9 & $1.412(3)$ \\
\hline C4-C5 & $1.419(3)$ & C5-C6 & $1.367(3)$ \\
\hline C6-C7 & $1.413(3)$ & C7-C8 & $1.384(3)$ \\
\hline C8-C9 & $1.423(3)$ & C10-C11 & $1.511(3)$ \\
\hline C11-C16 & $1.397(3)$ & $\mathrm{C} 11-\mathrm{C} 12$ & $1.399(3)$ \\
\hline C12-C13 & $1.385(3)$ & C13-C14 & $1.389(3)$ \\
\hline C14-C15 & $1.387(3)$ & C15-C16 & $1.386(3)$ \\
\hline C17-C18 & $1.403(3)$ & C18-C19 & $1.369(3)$ \\
\hline C19-C20 & $1.412(3)$ & $\mathrm{C} 20-\mathrm{C} 25$ & $1.409(3)$ \\
\hline C20-C21 & $1.418(3)$ & $\mathrm{C} 21-\mathrm{C} 22$ & $1.370(3)$ \\
\hline C22-C23 & $1.410(3)$ & $\mathrm{C} 23-\mathrm{C} 24$ & $1.383(3)$ \\
\hline C24-C25 & $1.421(3)$ & C26-C27 & $1.513(3)$ \\
\hline C27-C28 & $1.392(3)$ & C27-C32 & $1.394(3)$ \\
\hline C28-C29 & $1.383(3)$ & C29-C30 & $1.391(3)$ \\
\hline C30-C31 & $1.386(3)$ & C31-C32 & $1.388(3)$ \\
\hline C33-C11 & $1.769(2)$ & $\mathrm{C} 33-\mathrm{Cl} 2$ & $1.776(2)$ \\
\hline
\end{tabular}

Bond angles $\left({ }^{\circ}\right)$ for $\left[\left(\mathrm{C}_{16} \mathrm{H}_{10} \mathrm{~N}_{4} \mathrm{O}\right) \mathrm{Ni}\right]_{2} \cdot \mathrm{CH}_{2} \mathrm{Cl}_{2}(8 \mathrm{a})$. 


$\begin{array}{llll}\text { N8-Ni1-N3 } & 91.11(7) & \text { N8-Ni1-N1 } & 91.61(7) \\ \text { N3-Ni1-N1 } & 176.39(7) & \text { N8-Ni1-N2 } & 176.23(7) \\ \text { N3-Ni1-N2 } & 92.56(7) & \text { N1-Ni1-N2 } & 84.77(7) \\ \text { N4-Ni2-N7 } & 90.99(7) & \text { N4-Ni2-N6 } & 174.70(7) \\ \text { N7-Ni2-N6 } & 93.07(7) & \text { N4-Ni2-N5 } & 92.02(7) \\ \text { N7-Ni2-N5 } & 173.64(7) & \text { N6-Ni2-N5 } & 84.29(7) \\ \text { C1-N1-C9 } & 119.44(18) & \text { C1-N1-Ni1 } & 128.53(15) \\ \text { C9-N1-Ni1 } & 111.82(13) & \text { C10-N2-C8 } & 120.05(17) \\ \text { C10-N2-Ni1 } & 127.76(14) & \text { C8-N2-Ni1 } & 111.43(13) \\ \text { N4-N3-C16 } & 115.16(16) & \text { N4-N3-Ni1 } & 128.01(14) \\ \text { C16-N3-Ni1 } & 116.83(13) & \text { N3-N4-Ni2 } & 119.21(14) \\ \text { C17-N5-C25 } & 119.41(18) & \text { C17-N5-Ni2 } & 128.36(15) \\ \text { C25-N5-Ni2 } & 112.10(13) & \text { C26-N6-C24 } & 120.24(17) \\ \text { C26-N6-Ni2 } & 126.06(15) & \text { C24-N6-Ni2 } & 112.21(13) \\ \text { N8-N7-C32 } & 116.80(17) & \text { N8-N7-Ni2 } & 127.46(14) \\ \text { C32-N7-Ni2 } & 115.72(13) & \text { N7-N8-Ni1 } & 119.45(14) \\ \text { N1-C1-C2 } & 121.8(2) & \text { C3-C2-C1 } & 119.7(2) \\ \text { C2-C3-C4 } & 120.0(2) & \text { C9-C4-C3 } & 117.06(19) \\ \text { C9-C4-C5 } & 118.19(19) & \text { C3-C4-C5 } & 124.7(2) \\ \text { C6-C5-C4 } & 118.9(2) & \text { C5-C6-C7 } & 122.7(2) \\ \text { C8-C7-C6 } & 120.10(19) & \text { C7-C8-N2 } & 129.43(19) \\ \text { C1-C9-C9 } & 117.39(18) & \text { N2-C8-C9 } & 113.18(17) \\ \text { N1-C12 } & 116.79(19) & \text { C16-C11-C10 } & 125.93(18)\end{array}$




$\begin{array}{llll}\text { C12-C11-C10 } & 117.26(18) & \text { C13-C12-C11 } & 121.8(2) \\ \text { C12-C13-C14 } & 120.09(19) & \text { C15-C14-C13 } & 119.37(19) \\ \text { C16-C15-C14 } & 119.86(19) & \text { C15-C16-C11 } & 122.04(18) \\ \text { C15-C16-N3 } & 117.96(17) & \text { C11-C16-N3 } & 119.98(18) \\ \text { N5-C17-C18 } & 121.7(2) & \text { C19-C18-C17 } & 119.9(2) \\ \text { C18-C19-C20 } & 119.9(2) & \text { C25-C20-C19 } & 117.17(19) \\ \text { C25-C20-C21 } & 117.9(2) & \text { C19-C20-C21 } & 124.9(2) \\ \text { C22-C21-C20 } & 119.4(2) & \text { C21-C22-C23 } & 122.3(2) \\ \text { C24-C23-C22 } & 120.0(2) & \text { C23-C24-N6 } & 129.3(2) \\ \text { C23-C24-C25 } & 117.9(2) & \text { N6-C24-C25 } & 112.79(18) \\ \text { N5-C25-C20 } & 121.90(19) & \text { N5-C25-C24 } & 115.81(18) \\ \text { C20-C25-C24 } & 122.27(19) & \text { O2-C26-N6 } & 126.1(2) \\ \text { O2-C26-C27 } & 117.97(19) & \text { N6-C26-C27 } & 115.84(17) \\ \text { C28-C27-C32 } & 117.2(2) & \text { C28-C27-C26 } & 116.58(19) \\ \text { C32-C27-C26 } & 126.21(18) & \text { C29-C28-C27 } & 122.3(2) \\ \text { C28-C29-C30 } & 119.2(2) & \text { C31-C30-C29 } & 119.7(2) \\ \text { C30-C31-C32 } & 120.0(2) & \text { C31-C32-C27 } & 121.36(19) \\ \text { C31-C32-N7 } & 118.01(18) & \text { C27-C32-N7 } & 120.50(18) \\ \text { C11-C33-C12 } & 111.59(12) & & \end{array}$

Anisotropic atomic displacement parameters $\left(\AA^{2}\right)$ for $\left[\left(\mathrm{C}_{16} \mathrm{H}_{10} \mathrm{~N}_{4} \mathrm{O}\right) \mathrm{Ni}_{2} \cdot \mathrm{CH}_{2} \mathrm{Cl}_{2}(8 \mathrm{a})\right.$. The anisotropic atomic displacement factor exponent takes the form: $-2 \pi^{2}\left[h^{2} a^{* 2} U_{11}+\ldots+2 h k^{*} b^{*} U_{12}\right]$.

$$
\begin{array}{llllll}
\mathrm{U}_{11} & \mathrm{U}_{22} & \mathrm{U}_{33} & \mathrm{U}_{23} & \mathrm{U}_{13} & \mathrm{U}_{12}
\end{array}
$$

Ni1 $0.01075(13) 0.00827(12) 0.01414(14) \quad 0.0004(1) \quad 0.00386(10)-0.0005(1)$

Ni2 $0.01095(13) 0.00888(12) 0.01419(14)-0.0003(1) \quad 0.00273(10)-0.0015(1)$ 


$$
\begin{array}{llllll}
\mathrm{U}_{11} & \mathrm{U}_{22} & \mathrm{U}_{33} & \mathrm{U}_{23} & \mathrm{U}_{13} & \mathrm{U}_{12}
\end{array}
$$

$\begin{array}{lllllll}\mathrm{O} 1 & 0.0212(8) & 0.0122(7) & 0.0247(9) & 0.0004(6) & 0.0120(7) & -0.0041(6)\end{array}$

$\begin{array}{llllllll}\mathrm{O} 2 & 0.0255(9) & 0.0155(8) & 0.0276(9) & -0.0030(7) & 0.0037(7) & -0.0093(7)\end{array}$

$\begin{array}{lllllll}\mathrm{N} 1 & 0.0125(9) & 0.0124(8) & 0.0144(9) & -0.0011(7) & 0.0014(7) & -0.0013(7)\end{array}$

$\begin{array}{lllllll}\mathrm{N} 2 & 0.0126(9) & 0.0107(8) & 0.0132(9) & 0.0000(7) & 0.0042(7) & -0.0001(7)\end{array}$

$\begin{array}{lllllll}\mathrm{N} 3 & 0.0122(9) & 0.0099(8) & 0.0122(9) & 0.0005(7) & 0.0035(7) & -0.0012(7)\end{array}$

$\begin{array}{lllllll}\mathrm{N} 4 & 0.0125(9) & 0.0130(8) & 0.0131(9) & 0.0005(7) & 0.0031(7) & -0.0017(7)\end{array}$

$\begin{array}{lllllll}\mathrm{N} 5 & 0.0103(9) & 0.0147(9) & 0.0160(9) & 0.0000(7) & 0.0052(7) & -0.0014(7)\end{array}$

$\begin{array}{llllllll}\mathrm{N} 6 & 0.0111(9) & 0.0114(8) & 0.0165(9) & -0.0020(7) & 0.0028(7) & -0.0014(7)\end{array}$

$\begin{array}{lllllll}\mathrm{N} 7 & 0.0140(9) & 0.0095(8) & 0.0141(9) & -0.0020(7) & 0.0058(7) & -0.0025(7)\end{array}$

$\begin{array}{lllllll}\mathrm{N} 8 & 0.0145(9) & 0.0119(8) & 0.0142(9) & -0.0020(7) & 0.0049(7) & -0.0001(7)\end{array}$

$\begin{array}{lllllll}\text { C1 } & 0.0187(11) & 0.0130(10) & 0.0200(12) & 0.0025(9) & 0.0040(9) & 0.0007(8)\end{array}$

$\begin{array}{lllllll}\mathrm{C} 2 & 0.0244(13) & 0.0138(11) & 0.0293(13) & 0.0011(9) & 0.0050(11) & 0.0053(9)\end{array}$

$\begin{array}{lllllll}\text { C3 } & 0.0169(11) & 0.0162(11) & 0.0277(13) & -0.0044(9) & 0.0048(10) & 0.0050(9)\end{array}$

$\begin{array}{lllllll}\text { C4 } & 0.0129(10) & 0.0159(10) & 0.0185(11) & -0.0046(9) & 0.0015(9) & -0.0010(8)\end{array}$

$\begin{array}{lllllll}\text { C5 } & 0.0118(11) & 0.0225(11) & 0.0174(11) & -0.0062(9) & 0.0035(9) & -0.0004(9)\end{array}$

$\begin{array}{lllllll}\text { C6 } & 0.0143(11) & 0.0235(12) & 0.0156(11) & -0.0032(9) & 0.0052(9) & -0.0047(9)\end{array}$

$\begin{array}{lllllll}\text { C7 } & 0.0129(10) & 0.0151(10) & 0.0139(11) & -0.0007(8) & 0.0014(9) & -0.0025(8)\end{array}$

$\begin{array}{lllllll}\text { C8 } & 0.0099(10) & 0.0154(10) & 0.0112(10) & -0.0035(8) & -0.0002(8) & -0.0016(8)\end{array}$

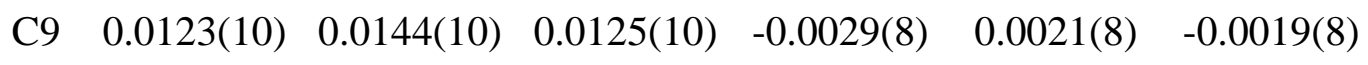

$\begin{array}{lllllll}\mathrm{C} 10 & 0.0115(10) & 0.0127(9) & 0.0139(10) & 0.0004(8) & 0.0010(8) & -0.0007(8)\end{array}$

$\begin{array}{lllllll}\mathrm{C} 11 & 0.0121(10) & 0.0107(9) & 0.0129(10) & 0.0025(8) & 0.0017(8) & 0.0015(8)\end{array}$

$\begin{array}{llllllll}\mathrm{C} 12 & 0.0159(11) & 0.0105(10) & 0.0183(11) & 0.0006(8) & 0.0018(9) & -0.0022(8)\end{array}$

$\begin{array}{lllllll}\text { C13 } & 0.0189(11) & 0.0097(10) & 0.0188(11) & -0.0030(8) & 0.0015(9) & -0.0006(8)\end{array}$

$\begin{array}{lllllll}\mathrm{C} 14 & 0.0181(11) & 0.0146(10) & 0.0167(11) & -0.0023(8) & 0.0049(9) & 0.0038(8)\end{array}$

$\begin{array}{lllllll}\mathrm{C} 15 & 0.0105(10) & 0.0144(10) & 0.0168(11) & 0.0009(8) & 0.0046(9) & -0.0010(8)\end{array}$ 

$\mathrm{U}_{11}$
$\mathrm{U}_{22}$
$\mathrm{U}_{33}$
$\mathrm{U}_{23}$
$\mathrm{U}_{13}$
$\mathrm{U}_{12}$
$\begin{array}{lllllll}\mathrm{C} 16 & 0.0122(10) & 0.0094(9) & 0.0135(10) & 0.0013(8) & 0.0008(8) & 0.0000(8)\end{array}$
$\begin{array}{lllllll}\text { C17 } & 0.0134(10) & 0.0147(10) & 0.0202(11) & 0.0004(9) & 0.0074(9) & -0.0011(8)\end{array}$
$\begin{array}{lllllll}\mathrm{C} 18 & 0.0145(11) & 0.0190(11) & 0.0224(12) & 0.0065(9) & 0.0082(9) & 0.0002(9)\end{array}$
$\begin{array}{lllllll}\text { C19 } & 0.0133(11) & 0.0257(12) & 0.0181(11) & 0.0066(9) & 0.0060(9) & 0.0018(9)\end{array}$
$\begin{array}{lllllll}\mathrm{C} 20 & 0.0093(10) & 0.0261(12) & 0.0150(11) & -0.0001(9) & 0.0049(9) & -0.0001(9)\end{array}$
$\begin{array}{lllllll}\mathrm{C} 21 & 0.0130(11) & 0.0328(13) & 0.0159(11) & -0.0026(10) & 0.0029(9) & -0.0009(9)\end{array}$
$\begin{array}{lllllll}\text { C22 } & 0.0133(11) & 0.0295(13) & 0.0207(12) & -0.0097(10) & 0.0029(9) & -0.0030(9)\end{array}$
$\begin{array}{lllllll}\mathrm{C} 23 & 0.0112(10) & 0.0202(11) & 0.0238(12) & -0.0058(9) & 0.0046(9) & -0.0002(9)\end{array}$
$\begin{array}{lllllll}\text { C24 } & 0.0090(10) & 0.0185(11) & 0.0180(11) & -0.0023(9) & 0.0047(9) & 0.0008(8)\end{array}$
$\begin{array}{lllllll}\text { C25 } & 0.0082(10) & 0.0170(10) & 0.0156(10) & -0.0012(9) & 0.0058(8) & -0.0003(8)\end{array}$
$\begin{array}{lllllll}\mathrm{C} 26 & 0.0117(10) & 0.0128(10) & 0.0227(12) & -0.0022(9) & 0.0061(9) & -0.0010(8)\end{array}$
$\begin{array}{lllllll}\mathrm{C} 27 & 0.0148(11) & 0.0106(10) & 0.0216(12) & -0.0001(8) & 0.0083(9) & 0.0017(8)\end{array}$
$\begin{array}{lllllll}\mathrm{C} 28 & 0.0167(11) & 0.0131(10) & 0.0287(13) & 0.0019(9) & 0.0076(10) & -0.0021(9)\end{array}$
$\begin{array}{lllllll}\text { C29 } & 0.0267(13) & 0.0123(11) & 0.0293(13) & 0.0046(9) & 0.0164(11) & 0.0009(9)\end{array}$
$\begin{array}{lllllll}\mathrm{C} 30 & 0.0334(14) & 0.0188(11) & 0.0186(12) & 0.0022(9) & 0.0099(10) & 0.0000(10)\end{array}$
$\begin{array}{lllllll}\mathrm{C} 31 & 0.0232(12) & 0.0156(11) & 0.0198(12) & 0.0013(9) & 0.0049(10) & -0.0032(9)\end{array}$
$\begin{array}{lllllll}\mathrm{C} 32 & 0.0155(11) & 0.0098(9) & 0.0167(11) & 0.0009(8) & 0.0060(9) & -0.0003(8)\end{array}$
$\begin{array}{llllllll}\mathrm{C} 33 & 0.0263(13) & 0.0151(11) & 0.0342(14) & -0.0012(10) & 0.0111(11) & 0.0023(9)\end{array}$
$\begin{array}{lllllll}\text { Cl1 } & 0.0348(4) & 0.0289(3) & 0.0366(4) & -0.0098(3) & -0.0002(3) & 0.0104(3)\end{array}$
$\begin{array}{lllllll}\mathrm{C} 2 & 0.0267(3) & 0.0210(3) & 0.0350(3) & -0.0011(2) & 0.0038(3) & -0.0063(2)\end{array}$ 
Hydrogen atom coordinates and isotropic atomic displacement parameters $\left(\AA^{2}\right)$ for $\left[\left(\mathrm{C}_{16} \mathrm{H}_{10} \mathrm{~N}_{4} \mathrm{O}\right) \mathrm{Ni}\right]_{2} \cdot \mathrm{CH}_{2} \mathrm{Cl}_{2}(8 \mathrm{a})$.

\begin{tabular}{|c|c|c|c|c|}
\hline & $\mathrm{x} / \mathrm{a}$ & $y / b$ & $\mathrm{z} / \mathrm{c}$ & $\mathrm{U}(\mathrm{eq})$ \\
\hline $\mathrm{H} 1$ & 0.5753 & 0.0948 & 0.4713 & 0.021 \\
\hline $\mathrm{H} 2$ & 0.7089 & 0.0383 & 0.4077 & 0.027 \\
\hline H3 & 0.8088 & 0.0679 & 0.2513 & 0.024 \\
\hline H5 & 0.8430 & 0.1444 & 0.0898 & 0.021 \\
\hline H6 & 0.8041 & 0.2318 & 0.0173 & 0.021 \\
\hline $\mathrm{H} 7$ & 0.6890 & 0.2884 & 0.1136 & 0.017 \\
\hline H12 & 0.5945 & 0.4065 & 0.4352 & 0.018 \\
\hline H13 & 0.5021 & 0.4478 & 0.5852 & 0.019 \\
\hline H14 & 0.3581 & 0.4022 & 0.6593 & 0.02 \\
\hline H15 & 0.3014 & 0.3167 & 0.5714 & 0.016 \\
\hline H17 & 0.1399 & 0.3004 & 0.1853 & 0.019 \\
\hline H18 & 0.0240 & 0.3321 & -0.0171 & 0.022 \\
\hline H19 & -0.0586 & 0.2718 & -0.1857 & 0.022 \\
\hline $\mathrm{H} 21$ & -0.0913 & 0.1719 & -0.2607 & 0.025 \\
\hline $\mathrm{H} 22$ & -0.0574 & 0.0823 & -0.2045 & 0.026 \\
\hline $\mathrm{H} 23$ & 0.0411 & 0.0558 & 0.0123 & 0.022 \\
\hline $\mathrm{H} 28$ & 0.0543 & 0.0271 & 0.4816 & 0.023 \\
\hline H29 & 0.1137 & 0.0262 & 0.7164 & 0.025 \\
\hline H30 & 0.2757 & 0.0750 & 0.8216 & 0.028 \\
\hline H31 & 0.3686 & 0.1282 & 0.6902 & 0.023 \\
\hline H33A & 0.7832 & 0.4737 & 0.2198 & 0.029 \\
\hline H33B & 0.7068 & 0.4258 & 0.2564 & 0.029 \\
\hline
\end{tabular}


Synthesis of 2-(quinolin-8-yl)-2,3-dihydro-1H-isoindole-1,3-dione (9a). A round bottom flask was charged with phthalic anhydride $(0.74 \mathrm{~g}, 5 \mathrm{mmol})$ and 8aminoquinoline $(0.72 \mathrm{~g}, 5 \mathrm{mmol})$. Acetic acid $(30 \mathrm{~mL})$ was added and the mixture was refluxed for 2 hours. The round bottom flask was allowed to cool to room temperature and an equal volume of water $(30 \mathrm{~mL})$ was added. The mixture was allowed to sit overnight and the solid was recovered by filtration. The solid was dried by high vacuum to yield 2-(quinolin-8-yl)-2,3-dihydro-1H-isoindole-1,3-dione in $987 \mathrm{mg}$ (3.60 mmol, 72 \%). ${ }^{1} \mathrm{H} \mathrm{NMR}\left(400 \mathrm{MHz}, \mathrm{CDCl}_{3}\right) \delta 8.87(\mathrm{~d}, J=4.2 \mathrm{~Hz}, 1 \mathrm{H}), 8.24(\mathrm{dd}, J=8.3,1.5 \mathrm{~Hz}$, 1H), $8.04-7.94(\mathrm{~m}, 3 \mathrm{H}), 7.81(\mathrm{ddd}, J=5.4,3.1,1.0 \mathrm{~Hz}, 2 \mathrm{H}), 7.76(\mathrm{~d}, J=7.3 \mathrm{~Hz}, 1 \mathrm{H})$, $7.68(\mathrm{t}, J=7.7 \mathrm{~Hz}, 1 \mathrm{H}), 7.45(\mathrm{dd}, J=8.3,4.2 \mathrm{~Hz}, 1 \mathrm{H}) .{ }^{13} \mathrm{C} \mathrm{NMR}\left(100 \mathrm{MHz}, \mathrm{CDCl}_{3}\right) \delta$ $168.07,151.05,144.39,136.45,134.30$, 132.62, 130.46, 129.88, 129.78, 129.47, 126.33, 123.99, 122.07. The spectral data is consistent with literature. ${ }^{98}$

Synthesis of intermediate (10a). In a $\mathrm{N}_{2}$ filled glovebox, an over dried $20 \mathrm{~mL}$ vial with a stir bar was charged with phthalimide (9a) $(274 \mathrm{mg}, 1.00 \mathrm{mmol})$ and THF (20 mL). In an oven dried $100 \mathrm{~mL}$ RB flask was charged with THF $(15 \mathrm{~mL})$ and $\mathrm{Ni}(\mathrm{COD})_{2}$. Both solutions were allowed to stir until starting materials were dissolved. The phthalimide containing solution was then added dropwise to the $\mathrm{Ni}(\mathrm{COD})_{2}(275 \mathrm{mg}, 1.00 \mathrm{mmol})$ solution while stirring vigorously. After complete addition of phthalimide solution, the reaction mixture was allowed to stir for $3 \mathrm{~h}$ at room temperature. After completion, the reaction mixture was filtered in a Buchner funnel with filter paper and washed with $\mathrm{Et}_{2} \mathrm{O}$ $(\sim 50 \mathrm{~mL})$. The filtrate was then added to a dried $\mathrm{RB}$ flask with $\mathrm{Et}_{2} \mathrm{O}$ and allowed to sit over night to allow any remaining nickel metallacycle to crash out. The filtrate was filtered the following morning. The nickel metallacycle was then dried under vacuum for $2 \mathrm{~h}$ yielding 10a in $256 \mathrm{mg}$ as light red solid (0.77 mmol, 77\%). ${ }^{1} \mathrm{H}$ NMR (400 $\mathrm{MHz}$, $\left.\mathrm{CD}_{2} \mathrm{Cl}_{2}\right) \delta 9.08(\mathrm{~d}, J=8.0 \mathrm{~Hz}, 1 \mathrm{H}), 8.45(\mathrm{~d}, J=7.9 \mathrm{~Hz}, 1 \mathrm{H}), 8.31(\mathrm{~d}, J=9.0 \mathrm{~Hz}, 1 \mathrm{H})$, $7.95(\mathrm{~d}, J=4.2 \mathrm{~Hz}, 1 \mathrm{H}), 7.74(\mathrm{t}, J=7.6 \mathrm{~Hz}, 1 \mathrm{H}), 7.58(\mathrm{t}, J=8.0 \mathrm{~Hz}, 1 \mathrm{H}), 7.50-7.38(\mathrm{~m}$, $3 \mathrm{H}), 7.33(\mathrm{dd}, J=8.3,4.9 \mathrm{~Hz}, 1 \mathrm{H}) .{ }^{13} \mathrm{C}\left\{{ }^{1} \mathrm{H}\right\} \mathrm{NMR}\left(100 \mathrm{MHz}, \mathrm{CD}_{2} \mathrm{Cl}_{2}\right) \delta 268.82,167.23$, $148.98,147.47,143.44,139.60,139.55,138.20$, 138.16, 134.01, 131.08, 129.53, 129.00, 
124.03, 121.90, 120.56, 119.84. FTIR (ATR, $\left.\mathrm{cm}^{-1}\right): 3041,1609,1575,1557,1498,1463$, $1398,1377,1338,1314,1263,751$.

Synthesis of Ni(II) metallacycle by decarbonylation (11a). In a glovebox filled with $\mathrm{N}_{2}$, the nickel metallacycle (10a) (332 $\mathrm{mg}, 1.00 \mathrm{mmol}$ ) was added to an oven dried 40 $\mathrm{mL}$ pressure tube with a stir bar. The $\mathrm{PhCH}_{3}(20 \mathrm{ml})$ was added and the pressure tube was closed with a Teflon screw cap. The reaction mixture was then taken out of the glovebox and placed in a pre-heated oil bath at $160{ }^{\circ} \mathrm{C}$ for $2 \mathrm{~h}$. After completion, the reaction mixture was taken out of the oil bath and allowed to cool to room temperature. The solution was allowed to sit undisturbed for a week while bright yellow crystals formed in solution. After the allotted time, the pressure tube was opened and the bright yellow crystals were isolated yielding 11a in $33.4 \mathrm{mg}(0.100 \mathrm{mmol}, 10 \%)$. ${ }^{1} \mathrm{H}$ NMR $(400 \mathrm{MHz}$, $\left.\mathrm{CD}_{2} \mathrm{Cl}_{2}\right) \delta 8.77(\mathrm{~d}, J=7.8 \mathrm{~Hz}, 1 \mathrm{H}), 8.55(\mathrm{~d}, J=3.2 \mathrm{~Hz}, 1 \mathrm{H}), 8.37(\mathrm{~d}, J=8.4 \mathrm{~Hz}, 1 \mathrm{H})$, $7.56(\mathrm{t}, J=7.9 \mathrm{~Hz}, 1 \mathrm{H}), 7.50(\mathrm{dd}, J=8.3,4.9 \mathrm{~Hz}, 1 \mathrm{H}), 7.43(\mathrm{~d}, J=7.3 \mathrm{~Hz}, 1 \mathrm{H}), 7.37(\mathrm{~d}, J$ $=8.1 \mathrm{~Hz}, 1 \mathrm{H}), 7.23-7.16(\mathrm{~m}, 1 \mathrm{H}), 7.12(\mathrm{dd}, J=4.7,1.2 \mathrm{~Hz}, 2 \mathrm{H}) .{ }^{13} \mathrm{C}\left\{{ }^{1} \mathrm{H}\right\} \operatorname{NMR}(100$ $\left.\mathrm{MHz}, \mathrm{CD}_{2} \mathrm{Cl}_{2}\right) \delta 186.96,150.18,147.46,147.02,146.70,145.48,140.29,137.75,131.76$, 130.59, 130.01, 128.01, 127.18, 122.45, 119.35, 118.66. FTIR (ATR, $\mathrm{cm}^{-1}$ ): 3050, 2063, 1634, 1573, 1501, 1466, 1385, 1340, 780. Elemental Analysis: calculated $\mathrm{C}_{17} \mathrm{H}_{10} \mathrm{~N}_{2} \mathrm{NiO}_{2}$, C: 61.32; H: 3.03; N: 8.41; found, C: 61.37; H: 3.06; N: 8.48.

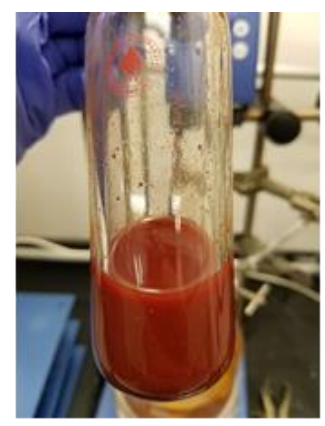

Before heating complex 10a in $\mathrm{PhCH}_{3}$.

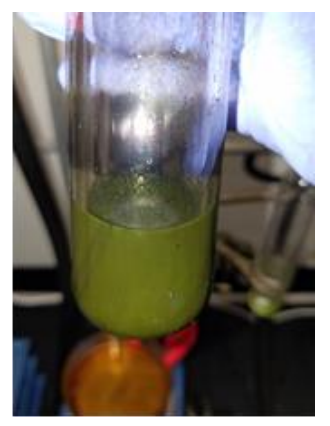

After heating complex 10a in $\mathrm{PhCH}_{3}$.

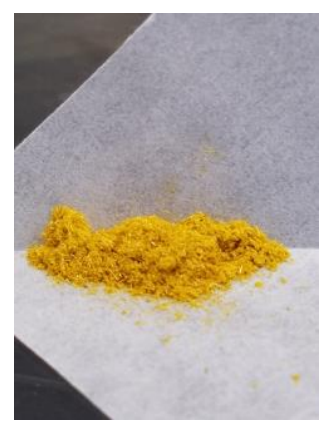

Crystals of complex 11a. 


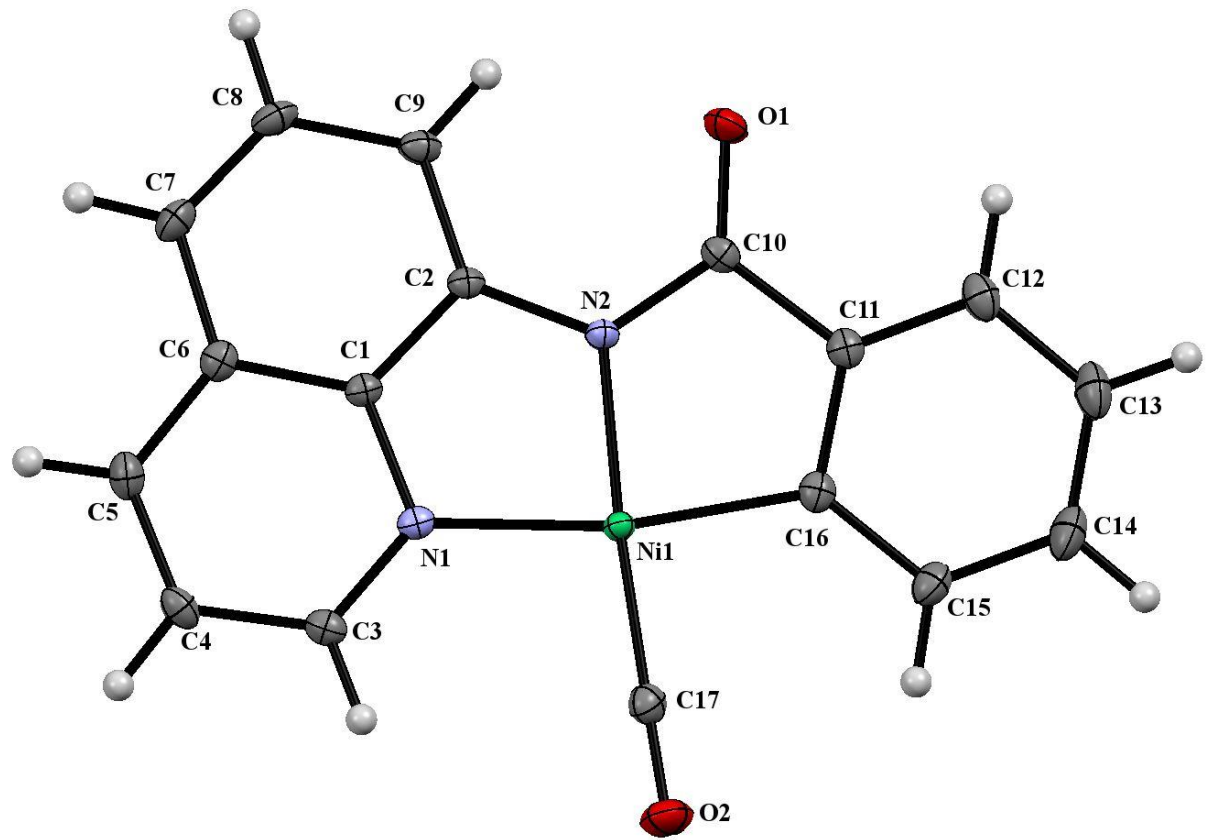

Perspective view of the molecular structure of $\left(\mathrm{C}_{16} \mathrm{H}_{10} \mathrm{~N}_{2} \mathrm{O}\right) \mathrm{Ni}(\mathrm{CO})$ (11a) with the atom labeling scheme for the non-hydrogen atoms. The thermal ellipsoids are scaled to enclose $50 \%$ probability.

\section{Description of the X-ray Structural Analysis of $\left(\mathrm{C}_{16} \mathrm{H}_{10} \mathrm{~N}_{2} \mathrm{O}\right) \mathrm{Ni}(\mathrm{CO})$ (11a).}

A long yellow parallelepiped crystal of $\left(\mathrm{C}_{16} \mathrm{H}_{10} \mathrm{~N}_{2} \mathrm{O}\right) \mathrm{Ni}(\mathrm{CO})$ (11a) was coated in polybutene oil (Sigma-Aldrich) and placed on the end of a MiTeGen loop. The sample was cooled to $100 \mathrm{~K}$ with an Oxford Cryostream 700 system and optically aligned on a Bruker AXS D8 Venture fixed-chi X-ray diffractometer equipped with a Triumph monochromator, a Mo K $\alpha$ radiation source $(\lambda=0.71073 \AA)$, and a PHOTON 100 CMOS detector. Three sets of 12 frames each were collected using the omega scan method with a 10 second exposure time. Integration of these frames followed by reflection indexing and least-squares refinement produced a crystal orientation matrix for the monoclinic crystal lattice that was used for the structural analysis.

Data collection consisted of the measurement of a total of 740 frames in four runs using omega scans with the detector held at $5.00 \mathrm{~cm}$ from the crystal. Frame scan parameters are summarized in Table 1 below:

Data collection details for $\left(\mathrm{C}_{16} \mathrm{H}_{10} \mathrm{~N}_{2} \mathrm{O}\right) \mathrm{Ni}(\mathrm{CO})$ (11a). 


\begin{tabular}{|c|c|c|c|c|c|c|c|}
\hline Run & $2 \theta$ & $\omega$ & $\varphi$ & $\chi$ & Scan Width $\left(^{\circ}\right)$ & Frames & $\begin{array}{c}\text { Exposure } \\
\text { Time }(\mathrm{sec})\end{array}$ \\
\hline 1 & 16.35 & -166.34 & -156.00 & 54.74 & 1.00 & 185 & 60.00 \\
\hline 2 & 16.35 & -166.34 & -54.00 & 54.74 & 1.00 & 185 & 60.00 \\
\hline 3 & 16.35 & -166.34 & -105.00 & 54.74 & 1.00 & 185 & 60.00 \\
\hline 4 & 16.35 & -166.34 & 153.00 & 54.74 & 1.00 & 185 & 60.00 \\
\hline
\end{tabular}

The APEX3 software program (version 2016.9-0) ${ }^{100}$ was used for diffractometer control, preliminary frame scans, indexing, orientation matrix calculations, least-squares refinement of cell parameters, and the data collection. The frames were integrated with the Bruker SAINT software package using a narrow-frame algorithm. The integration of the data using a monoclinic unit cell yielded a total of 29130 reflections to a maximum $\theta$ angle of $30.09^{\circ}(0.71 \AA$ resolution), of which 3891 were independent (average redundancy 7.487, completeness $\left.=99.8 \%, \mathrm{R}_{\mathrm{int}}=3.05 \%, \mathrm{R}_{\mathrm{sig}}=1.96 \%\right)$ and 3417 $(87.82 \%)$ were greater than $2 \sigma\left(\mathrm{F}^{2}\right)$. The final cell constants of $\underline{\mathrm{a}}=10.9298(5) \AA \mathrm{A}, \underline{\mathrm{b}}=$ 7.1659(3) $\AA, \underline{c}=17.2345(7) \AA, \beta=101.0570(10)^{\circ}$, volume $=1324.78(10) \AA^{3}$, are based upon the refinement of the XYZ-centroids of 9902 reflections above $20 \sigma(\mathrm{I})$ with $6.174^{\circ}$ $<2 \theta<60.11^{\circ}$. Data were corrected for absorption effects using the multi-scan method (SADABS). The ratio of minimum to maximum apparent transmission was 0.859 . The calculated minimum and maximum transmission coefficients (based on crystal size) are 0.541 and 0.921 .

The structure was solved by using the intrinisic phasing routine available in the APEX3 software $^{100}$ and refined using the programs provided by SHELXL-2014/7. ${ }^{101}$ The crystallographic asymmetric unit consists of only a molecule of $\left(\mathrm{C}_{16} \mathrm{H}_{10} \mathrm{~N}_{2} \mathrm{O}\right) \mathrm{Ni}(\mathrm{CO})$ (13a). Idealized positions for the aromatic hydrogen atoms were included as fixed contributions using a riding model with isotropic temperature factors set at 1.2 times that of the adjacent carbon atom. Full-matrix least-squares refinement, based upon the minimization of $\Sigma \mathrm{w}_{\mathrm{i}}\left|\mathrm{F}_{\mathrm{o}}{ }^{2}-\mathrm{F}_{\mathrm{c}}{ }^{2}\right|^{2}$, with weighting $\mathrm{w}_{\mathrm{i}}^{-1}=\left[\sigma^{2}\left(\mathrm{~F}_{\mathrm{o}}{ }^{2}\right)+(0.0254 \mathrm{P})^{2}+1.0711 \mathrm{P}\right]$, where $\mathrm{P}=\left(\operatorname{Max}\left(\mathrm{F}_{\mathrm{o}}{ }^{2}, 0\right)+2 \mathrm{~F}_{\mathrm{c}}{ }^{2}\right) / 3 .{ }^{101}$ The final anisotropic full-matrix least-squares refinement on $\mathrm{F}^{2}$ with 199 variables converged at $\mathrm{R} 1=2.48 \%$ for the 3417 data with $\mathrm{I}>2 \sigma(\mathrm{I})$ and $w \mathrm{R} 2=6.11 \%$ for all data. The goodness-of-fit was $1.033 .{ }^{101}$ A correction for secondary extinction was not applied. The largest peak in the final difference electron 
density synthesis was $0.537 \mathrm{e}^{-} / \AA^{3}$ and the largest hole was $-0.290 \mathrm{e}^{-} / \AA^{3}$ with an RMS deviation of $0.063 \mathrm{e}^{-} / \AA^{3}$. The linear absorption coefficient, atomic scattering factors, and anomalous dispersion corrections were calculated from values found in the International Tables of X-ray Crystallography. ${ }^{103}$

\section{Crystal data for $\left(\mathrm{C}_{16} \mathrm{H}_{10} \mathrm{~N}_{2} \mathrm{O}\right) \mathrm{Ni}(\mathrm{CO})$ (11a).}

Ident. code jh54cms

Chem. form. $\mathrm{C}_{17} \mathrm{H}_{10} \mathrm{~N}_{2} \mathrm{NiO}_{2}$

Mol. weight $332.98 \mathrm{~g} / \mathrm{mol}$

Temperature 100(2) K

Wavelength $0.71073 \AA$

Crystal size 0.057 x 0.120 x $0.476 \mathrm{~mm}$

Cryst. type monoclinic

Space group $\mathrm{P} 2{ }_{1} / \mathrm{c}$ (No. 14)

Unit cell

$$
\begin{array}{ll}
\mathrm{a}=10.9298(5) \AA & \alpha=90^{\circ} \\
\mathrm{b}=7.1659(3) \AA & \beta=101.0570(10)^{\circ} \\
\mathrm{c}=17.2345(7) \AA & \gamma=90^{\circ}
\end{array}
$$

Volume, $\AA^{3} 1324.78(10)$

Z 4

Density $\quad 1.669 \mathrm{~g} / \mathrm{cm}^{3}$

(calc)

Abs. coeff. $1.472 \mathrm{~mm}^{-1}$

$\mathrm{F}(000) \quad 680$

\section{Data collection and structure refinement for $\left(\mathrm{C}_{16} \mathrm{H}_{10} \mathrm{~N}_{2} \mathrm{O}\right) \mathrm{Ni}(\mathrm{CO})$ (11a).}

Theta range

Index ranges

Reflections

Independent refls

Coverage

Abs. correction

Max./ min. trans.

Refinement method Full-matrix least-squares on $\mathrm{F}^{2}$

Ref. program

Data / restrs / parms
SHELXL-2014/7 (Sheldrick, 2014)

\subsection{9 to $30.09^{\circ}$}

$-15 \leq \mathrm{h} \leq 15,-10 \leq \mathrm{k} \leq 10,-22 \leq 1 \leq 24$

29130

$3891[\mathrm{R}(\mathrm{int})=0.0305]$

$99.8 \%$

multi-scan

$3891 / 0 / 199$ 
GOF on $\mathrm{F}^{2} \quad 1.033$

Final R indices $\quad \begin{array}{ll}3417 \text { data; } & \mathrm{I}>2 \sigma(\mathrm{I})\end{array}$

all data $\quad \mathrm{R} 1=0.0319, \mathrm{wR} 2=0.0611$

Largest diff. peak 0.537 and $-0.290 \mathrm{e}^{-} / \AA^{3}$ and hole

Atomic coordinates and equivalent isotropic atomic displacement parameters $\left(\AA^{2}\right)$ for $\left(\mathrm{C}_{16} \mathrm{H}_{10} \mathrm{~N}_{2} \mathrm{O}\right) \mathrm{Ni}(\mathrm{CO})$ (11a). $\mathrm{U}(\mathrm{eq})$ is defined as one third of the trace of the orthogonalized $U_{i j}$ tensor.

$\mathrm{x} / \mathrm{a} \quad \mathrm{y} / \mathrm{b} \quad \mathrm{z} / \mathrm{c} \quad \mathrm{U}(\mathrm{eq})$

$\begin{array}{lllll}\text { Ni1 } & 0.39088(2) & 0.71655(2) & 0.50851(2) & 0.00949(5) \\ \text { O1 } & 0.49582(10) & 0.57634(15) & 0.30799(6) & 0.0178(2) \\ \text { O2 } & 0.22921(10) & 0.71492(16) & 0.62317(7) & 0.0227(2) \\ \text { N1 } & 0.54072(10) & 0.81810(15) & 0.57288(6) & 0.0102(2) \\ \text { N2 } & 0.48904(10) & 0.69556(15) & 0.43209(6) & 0.0104(2) \\ \text { C1 } & 0.63842(12) & 0.82426(18) & 0.53303(7) & 0.0102(2) \\ \text { C2 } & 0.61147(12) & 0.75649(17) & 0.45385(7) & 0.0103(2) \\ \text { C3 } & 0.55955(12) & 0.88152(18) & 0.64668(8) & 0.0126(2) \\ \text { C4 } & 0.67458(13) & 0.95435(19) & 0.68502(8) & 0.0148(3) \\ \text { C5 } & 0.77259(13) & 0.9592(2) & 0.64618(8) & 0.0157(3) \\ \text { C6 } & 0.75693(12) & 0.89248(18) & 0.56750(8) & 0.0126(2) \\ \text { C7 } & 0.85140(13) & 0.8925(2) & 0.52158(9) & 0.0164(3) \\ \text { C8 } & 0.82440(13) & 0.8296(2) & 0.44511(9) & 0.0164(3) \\ \text { C9 } & 0.70522(13) & 0.76201(19) & 0.41003(8) & 0.0137(2) \\ \text { C10 } & 0.43969(12) & 0.61425(18) & 0.36133(7) & 0.0119(2) \\ \text { C11 } & 0.30521(12) & 0.57602(18) & 0.35883(8) & 0.0121(2)\end{array}$




$\begin{array}{ccccc} & \mathrm{x} / \mathrm{a} & \mathrm{y} / \mathrm{b} & \mathrm{z} / \mathrm{c} & \mathrm{U}(\mathrm{eq}) \\ \mathrm{C} 12 & 0.22833(13) & 0.5061(2) & 0.29162(8) & 0.0171(3) \\ \mathrm{C} 13 & 0.10260(14) & 0.4776(2) & 0.29229(9) & 0.0222(3) \\ \mathrm{C} 14 & 0.05619(13) & 0.5201(2) & 0.35963(10) & 0.0220(3) \\ \mathrm{C} 15 & 0.13410(13) & 0.5897(2) & 0.42726(9) & 0.0169(3) \\ \mathrm{C} 16 & 0.26083(12) & 0.61852(18) & 0.42801(8) & 0.0126(2) \\ \mathrm{C} 17 & 0.29259(13) & 0.7178(2) & 0.57821(8) & 0.0148(3)\end{array}$

Interatomic distances ( $\left(\right.$ ) for $\left(\mathrm{C}_{16} \mathrm{H}_{10} \mathrm{~N}_{2} \mathrm{O}\right) \mathrm{Ni}(\mathrm{CO})(11 \mathrm{a})$.

$\begin{array}{llll}\text { Ni1-C17 } & 1.7584(14) & \text { Ni1-N2 } & 1.8574(11) \\ \text { Ni1-C16 } & 1.9189(13) & \text { Ni1-N1 } & 1.9354(11) \\ \text { O1-C10 } & 1.2294(16) & \text { O2-C17 } & 1.1344(17) \\ \text { N1-C3 } & 1.3290(17) & \text { N1-C1 } & 1.3766(16) \\ \text { N2-C10 } & 1.3658(17) & \text { N2-C2 } & 1.3890(16) \\ \text { C1-C6 } & 1.4052(18) & \text { C1-C2 } & 1.4250(18) \\ \text { C2-C9 } & 1.3852(17) & \text { C3-C4 } & 1.4039(19) \\ \text { C4-C5 } & 1.368(2) & \text { C5-C6 } & 1.4168(19) \\ \text { C6-C7 } & 1.4163(19) & \text { C7-C8 } & 1.370(2) \\ \text { C8-C9 } & 1.4125(19) & \text { C10-C11 } & 1.4876(18) \\ \text { C11-C12 } & 1.3878(19) & \text { C11-C16 } & 1.4041(18) \\ \text { C12-C13 } & 1.392(2) & \text { C13-C14 } & 1.387(2) \\ \text { C14-C15 } & 1.397(2) & \text { C15-C16 } & 1.3980(19)\end{array}$

Bond angles $\left({ }^{\circ}\right)$ for $\left(\mathrm{C}_{16} \mathrm{H}_{10} \mathrm{~N}_{2} \mathrm{O}\right) \mathrm{Ni}(\mathrm{CO})$ (11a).

$\begin{array}{llll}\text { C17-Ni1-N2 } & 175.14(6) & \text { C17-Ni1-C16 } & 91.77(6) \\ \text { N2-Ni1-C16 } & 84.62(5) & \text { C17-Ni1-N1 } & 99.62(6)\end{array}$




\begin{tabular}{|c|c|c|c|}
\hline N2-Ni1-N1 & $84.06(5)$ & C16-Ni1-N1 & $168.56(5)$ \\
\hline C3-N1-C1 & $118.29(11)$ & C3-N1-Ni1 & $129.48(9)$ \\
\hline C1-N1-Ni1 & $112.22(8)$ & $\mathrm{C} 10-\mathrm{N} 2-\mathrm{C} 2$ & $124.76(11)$ \\
\hline C10-N2-Ni1 & 119.00(9) & C2-N2-Ni1 & $116.15(8)$ \\
\hline N1-C1-C6 & $122.72(12)$ & N1-C1-C2 & 115.41(11) \\
\hline C6-C1-C2 & $121.86(12)$ & $\mathrm{C} 9-\mathrm{C} 2-\mathrm{N} 2$ & $129.56(12)$ \\
\hline $\mathrm{C} 9-\mathrm{C} 2-\mathrm{C} 1$ & $118.27(12)$ & $\mathrm{N} 2-\mathrm{C} 2-\mathrm{C} 1$ & $112.16(11)$ \\
\hline N1-C3-C4 & $122.54(12)$ & $\mathrm{C} 5-\mathrm{C} 4-\mathrm{C} 3$ & $119.57(12)$ \\
\hline C4-C5-C6 & $119.84(13)$ & $\mathrm{C} 1-\mathrm{C} 6-\mathrm{C} 7$ & $118.40(12)$ \\
\hline C1-C6-C5 & $117.02(12)$ & C7-C6-C5 & $124.58(13)$ \\
\hline C8-C7-C6 & $119.45(13)$ & C7-C8-C9 & $122.37(13)$ \\
\hline $\mathrm{C} 2-\mathrm{C} 9-\mathrm{C} 8$ & 119.63(13) & O1-C10-N2 & $126.42(12)$ \\
\hline O1-C10-C11 & $124.72(12)$ & N2-C10-C11 & $108.86(11)$ \\
\hline C12-C11-C16 & $122.32(13)$ & C12-C11-C10 & $121.83(12)$ \\
\hline C16-C11-C10 & $115.84(11)$ & C11-C12-C13 & $119.13(14)$ \\
\hline C14-C13-C12 & $119.68(14)$ & C13-C14-C15 & $120.96(14)$ \\
\hline C14-C15-C16 & $120.32(14)$ & $\mathrm{C} 15-\mathrm{C} 16-\mathrm{C} 11$ & $117.59(12)$ \\
\hline C15-C16-Ni1 & $131.00(11)$ & C11-C16-Ni1 & 111.39(9) \\
\hline O2-C17-Ni1 & 178.66(13) & & \\
\hline
\end{tabular}


Anisotropic atomic displacement parameters $\left(\AA^{2}\right)$ for $\left(\mathrm{C}_{16} \mathrm{H}_{10} \mathrm{~N}_{2} \mathrm{O}\right) \mathrm{Ni}(\mathrm{CO})$ (11a). The anisotropic atomic displacement factor exponent takes the form: $2 \pi^{2}\left[h^{2} a^{* 2} U_{11}+\ldots+2 h k a^{*} b^{*} U_{12}\right]$.

$\begin{array}{llllll}\mathrm{U}_{11} & \mathrm{U}_{22} & \mathrm{U}_{33} & \mathrm{U}_{23} & \mathrm{U}_{13} & \mathrm{U}_{12}\end{array}$

$\begin{array}{lllllll}\text { Ni1 } & 0.00930(8) & 0.01046(8) & 0.00940(8) & -0.00005(6) & 0.00354(6) & -0.00031(6) \\ \text { O1 } & 0.0215(5) & 0.0220(5) & 0.0112(4) & -0.0029(4) & 0.0063(4) & -0.0029(4) \\ \text { O2 } & 0.0223(5) & 0.0265(6) & 0.0232(5) & -0.0018(5) & 0.0140(4) & -0.0033(5) \\ \text { N1 } & 0.0111(5) & 0.0088(5) & 0.0113(5) & 0.0018(4) & 0.0036(4) & 0.0011(4) \\ \text { N2 } & 0.0102(5) & 0.0123(5) & 0.0092(5) & 0.0002(4) & 0.0028(4) & -0.0007(4) \\ \text { C1 } & 0.0114(5) & 0.0082(5) & 0.0114(6) & 0.0016(4) & 0.0036(4) & 0.0006(4) \\ \text { C2 } & 0.0116(5) & 0.0088(6) & 0.0111(6) & 0.0008(4) & 0.0038(4) & 0.0010(4) \\ \text { C3 } & 0.0159(6) & 0.0108(6) & 0.0113(6) & 0.0012(5) & 0.0035(5) & 0.0018(5) \\ \text { C4 } & 0.0203(7) & 0.0129(6) & 0.0102(6) & 0.0002(5) & 0.0009(5) & -0.0007(5) \\ \text { C5 } & 0.0156(6) & 0.0145(6) & 0.0151(6) & 0.0007(5) & -0.0015(5) & -0.0027(5) \\ \text { C6 } & 0.0127(6) & 0.0103(6) & 0.0145(6) & 0.0024(5) & 0.0021(5) & -0.0002(5) \\ \text { C7 } & 0.0116(6) & 0.0169(6) & 0.0213(7) & 0.0013(5) & 0.0044(5) & -0.0024(5) \\ \text { C8 } & 0.0142(6) & 0.0159(6) & 0.0217(7) & 0.0014(5) & 0.0097(5) & -0.0007(5) \\ \text { C9 } & 0.0162(6) & 0.0121(6) & 0.0144(6) & -0.0002(5) & 0.0070(5) & -0.0008(5) \\ \text { C10 } & 0.0152(6) & 0.0103(6) & 0.0100(5) & 0.0015(4) & 0.0023(5) & 0.0002(5) \\ \text { C11 } & 0.0137(6) & 0.0092(6) & 0.0129(6) & 0.0014(5) & 0.0012(5) & 0.0006(5) \\ \text { C12 } & 0.0187(7) & 0.0153(6) & 0.0153(6) & -0.0001(5) & -0.0019(5) & 0.0007(5) \\ \text { C13 } & 0.0182(7) & 0.0213(7) & 0.0231(7) & -0.0024(6) & -0.0064(6) & 0.0002(6) \\ \text { C14 } & 0.0118(6) & 0.0208(7) & 0.0313(8) & -0.0004(6) & -0.0011(6) & -0.0008(5) \\ \text { C15 } & 0.0125(6) & 0.0158(6) & 0.0224(7) & 0.0004(5) & 0.0035(5) & 0.0006(5) \\ \text { C16 } & 0.0129(6) & 0.0094(6) & 0.0150(6) & 0.0012(5) & 0.0015(5) & 0.0002(5) \\ \text { 0.0151(6) } & 0.0128(6) & 0.0166(6) & -0.0010(5) & 0.0030(5) & -0.0014(5)\end{array}$



$\mathrm{U}_{11}$
$\mathrm{U}_{22}$
$\mathrm{U}_{33}$
$\mathrm{U}_{23}$
$\mathrm{U}_{13}$
$\mathrm{U}_{12}$

Hydrogen atom coordinates and isotropic atomic displacement parameters $\left(\AA^{2}\right)$ for $\left(\mathrm{C}_{16} \mathrm{H}_{10} \mathrm{~N}_{2} \mathrm{O}\right) \mathrm{Ni}(\mathrm{CO})$ (11a).

$\begin{array}{ccccc} & \mathrm{x} / \mathrm{a} & \mathrm{y} / \mathrm{b} & \mathrm{z} / \mathrm{c} & \mathrm{U}(\mathrm{eq}) \\ \mathrm{H} 3 & 0.4926 & 0.8774 & 0.6746 & 0.015 \\ \mathrm{H} 4 & 0.6843 & 1.0000 & 0.7376 & 0.018 \\ \mathrm{H} 5 & 0.8509 & 1.0071 & 0.6719 & 0.019 \\ \text { H7 } & 0.9328 & 0.9358 & 0.5436 & 0.02 \\ \text { H8 } & 0.8881 & 0.8316 & 0.4146 & 0.02 \\ \text { H9 } & 0.6894 & 0.7205 & 0.3567 & 0.016 \\ \text { H12 } & 0.2611 & 0.4780 & 0.2457 & 0.021 \\ \text { H13 } & 0.0488 & 0.4293 & 0.2468 & 0.027 \\ \text { H14 } & -0.0298 & 0.5016 & 0.3598 & 0.026 \\ \text { H15 } & 0.1008 & 0.6175 & 0.4730 & 0.02\end{array}$<smiles>O=[N+]([O-])c1ccccc1[As]</smiles>

Synthesis of (2-nitrophenyl)silver (12a). The title compound was prepared by following literature synthesis. ${ }^{104}$ To a mixture of $\mathrm{AgF}(269 \mathrm{mg}, 2.12 \mathrm{mmol})$ and anhydrous MeCN (20 $\mathrm{mL}$ ) in a glovebox filled with $\mathrm{N}_{2}$, was added a solution of 5,5-dimethyl-2-(2nitrophenyl)-[1,3,2]dioxaborinane (499 mg, $2.12 \mathrm{mmol}$ ) in $\mathrm{MeCN}$ (20 mL). The reaction mixture was stirred at room temperature for $24 \mathrm{~h}$ while protected from light with aluminum foil. The solid was collected by filtration and washed with diethyl ether $(\sim 50$ $\mathrm{mL})$, to yield $316 \mathrm{mg}(1.37 \mathrm{mmol}, 65 \%)$ as a bright yellow solid. ${ }^{1} \mathrm{H}$ NMR (400 MHz, 
DMSO- $\left.d_{6}\right) \delta 8.03(\mathrm{dd}, J=8.1,1.2 \mathrm{~Hz}, 1 \mathrm{H}), 7.95(\mathrm{dd}, J=7.0,1.7 \mathrm{~Hz}, 1 \mathrm{H}), 7.54(\mathrm{td}, J=$ $7.1,1.3 \mathrm{~Hz}, 1 \mathrm{H}), 7.40$ (td, $J=8.4,8.0,1.7 \mathrm{~Hz}, 1 \mathrm{H})$.

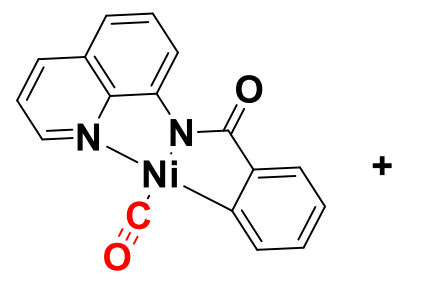

$11 \mathrm{a}$<smiles>O=[N+]([O-])c1ccccc1[Hg]</smiles>

$12 a$
DMA, r.t., 1 h

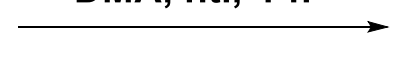

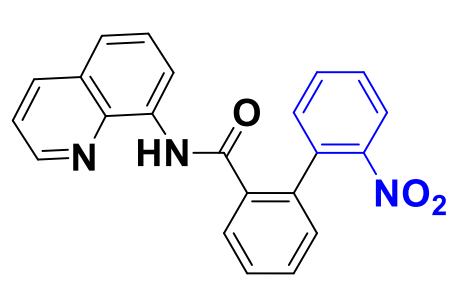

$13 a$

Procedure for (hetero)arylation of complex 11a with silver aryls. To a mixture of complex 11a $(8.3 \mathrm{mg}, 0.025 \mathrm{mmol})$ and anhydrous DMA $(1 \mathrm{~mL})$ in a glovebox filled with $\mathrm{N}_{2}$, was added a solution of (2-nitrophenyl)silver(I) $(0.025 \mathrm{mmol}$ or $0.050 \mathrm{mmol})$ in anhydrous DMA (1 mL) dropwise. The mixture was stirred at room temperature for $1 \mathrm{~h}$. The reaction mixture was taken out of the glovebox and poured into a $100 \mathrm{~mL}$ separatory funnel. To the solution, water $(25 \mathrm{~mL})$, aqueous $\mathrm{HCl}(2 \mathrm{~N}, 3 \mathrm{~mL})$ were added and extracted with ethyl acetate $(2 \times 30 \mathrm{~mL})$. The combined organic layers were washed with water $(3 \times 30 \mathrm{~mL})$ and brine $(\sim 15 \mathrm{~mL})$, dried over $\mathrm{Na}_{2} \mathrm{SO}_{4}$, filtered, and concentrated under vacuum. Then 1,3,5-trimethoxybenzene $(2.0 \mathrm{mg})$ was added to the residue and the crude mixture was dissolved in $\mathrm{CDCl}_{3}$ for ${ }^{1} \mathrm{H}$ analysis. 


\section{$\underline{\text { References }}$}

${ }^{1}$ (a) Bräse, S.; de Meijere, A. In Metal-catalyzed Cross-coupling Reactions; Diederich, F., Stang, P. J., Eds.; Wiley-VCH: Weinheim, p 99. (b) Beller, M.; Riermeier, T. H.; Stark,G. In Transition Metals for Organic Synthesis; Beller, M., Bolm,C., Eds.; WILEY-VCH: Weinheim, 1998; p 209.

2 (a) Miyaura, N.; Yamada, K.; Suzuki, A. Tetrahedron 1979, 20, 3437-3440. (b) Miyaura, N.; Suzuki, A. Chem. Commun. 1979, 1979, 866-870.

${ }^{3}$ Tamao, K.; Sumitani, K.; Kumada, M. J. Am. Chem. Soc. 1972, 94, 4374-4376.

${ }^{4}$ Heck, R. F.; Nolley, J. P. J. Org. Chem. 1972, 37, 2320-2322.

5 (a) Koning, P. D.; McAndrew, D.; Moore, R.; Moses, I. B.; Boyles, D. C.; Kissick, K.; Stanchina, C. L.; Cuthbertosn, T.; Kamatani, A.; Rahman, L.; Rodriguez, R.; Urbina, A.; Sandoval, A.; Rose, P. R. Org. Process Rev. Dev. 2011, 15, 1018-1026. (b) Martin, A. D.; Siamaki, A. R.; Belecki, K.; Gupton, B. F. J. Org. Chem. 2015, 80, 1915-1919. (c) Glasnv, T. N.; Kappe, C. O. Adv. Synth. Catal.2010, 352, 3089-3097.

${ }^{6}$ Nilsson, M.; Kulonen, E.; Sunner, S.; Frank, V.; Brunvoll, J.; Bunnenberg, F.; Djerassi, C.; Records, R. Acta Chem. Scand. 1966, 20, 423-426.

${ }^{7}$ Goossen, L. J.; Deng, G. J.; Levy, L. M. Science 2006, 313, 662-664.

${ }^{8}$ Voutchkova, A.; Coplin, A.; Leadbeater, N. E.; Crabtree, R. H. Chem. Commun. 2008, $0,6312-6314$.

${ }^{9}$ Cornella, J.; Lu, P.; Larrosa, I. Org. Lett. 2009, 11, 5506-5509.

${ }^{10}$ Xie, K.; Yang, Z.; Zhou, X.; Li, X.; Wang, S.; Tan, Z.; An, X.; Guo, C.-C. Org. Lett. 2010, 12, 1564-1567.

11 (a) Zhou, J.; Hu, P.; Zhang, M.; Huang, S.; Wang, M.; Su, W. Chem.-Eur. J. 2010, 16, 5876-5881. (b) Zhao, H.; Wei, Y.; Xu, J.; Kan, J.; Su, W.; Hong, M. J. Org. Chem. 2011, 76, 882-893. (c) Pei, K.; Jie, X.; Zhao, H.; Su, W. Eur. J. Org. Chem. 2014, 2014, 4230-4233. 
12 (a) Abbot, V.; Sharma, P.; Dhiman, S.; Noolvi, M. N.; Patel, H. M.; Bhardwaj, V. RSC $A d v$. 2017, 7, 28313-28349. (b) Moreno, L. M.; Quiroga, J.; Abonia, R.; RamirezPrada, J.; Insuasty, B. Molecules 2018, 23, 1956.

${ }^{13}$ (a) Lingaraju, G. S.; Swaroop, T. R.; Vinayaka, A. C.; Kumar, K. S. S.; Sadashiva, M. P.; Ragappa, K. S. Synthesis 2012, 44, 1373-1379. (b) Loy, N. S. Y.; Kim, S.; Park, C.M. Org. Lett. 2015, 17, 395-397. (c) Xiong, X.; Bagley, M. C.; Chapaneri, K. Tetrahedron Lett. 2004, 45, 6121-6124.

14 Zhang, F.; Greaney, M. F. Angew. Chem., Int. Ed. 2010, 49, 2768-2771.

${ }^{15} \mathrm{Hu}$, P.; Zhang, M.; Jie, X.; Su, W. Angew. Chem., Int. Ed. 2012, 51, 227-231.

${ }^{16}$ (a) Kan, J.; Huang, S.; Lin, J.; Zhang, M.; Su, W. Angew. Chem., Int. Ed. 2015, 54, 2199-2203. (b) Taylor, J. B.; Greaney, M. F. Chem. Commun. 2012, 48, 8270-8272. (c) Seo, S.; Slater, M.; Greaney, M. F. Org. Lett. 2012, 14, 2650-2653.

${ }^{17}$ (a) Chen, L.; Ju, L.; Bustin, K. A.; Hoover, J. M. Chem. Commun. 2015, 51, 15059-5062. (b) Patra, T.; Nandi, S.; Sahoo, S. K.; Maiti, D. Chem. Commun. 2016, 52, 14321435.

${ }^{18}$ Zhao, S.; Liu, Y.-J.; Yan, S.-Y.; Chen, F.-J.; Zhang, Z.-Z.; Shi, B.-F. Org. Lett. 2015, $17,3338-3341$.

${ }^{19}$ Wang, C.; Piel, I.; Glorius, F. J. Am. Chem. Soc. 2009, 131, 4194-4195.

${ }^{20}$ Yu, W. Y.; Sit, W. N.; Zhou, Z. Y.; Chan, A. S. C. Org. Lett. 2009, 11, 3174-3177.

${ }^{21}$ (a) Engle, K.M.; Mei, T.-S.; Wasa, M.; Yu, J.-Q. Acc. Chem. Res. 2012, 45, 788-802. (b) Daugulis, O.; Do, H.-Q.; Shabashov, D. Acc. Chem. Res. 2009, 42, 1074-1086. (c) Neufeldt, S. R.; Sanford, M. S. Acc. Chem. Res. 2012, 45, 936-946. (d) Lyons, T. W.; Sanford, M. S. Chem. Rev. 2010, 110, 1147-1169. (e) Ackermann, L. Acc. Chem. Res. 2014, 47, 281-295.

${ }^{22}$ (a) Giri, R.; Chen, X.; Yu, J.-Q. Angew. Chem. Int. Ed. 2005, 44, 2112-2115. (b) Desai, L. V.; Hull, K. L.; Sanford, M. S. J. Am. Chem. Soc. 2004, 126, 9542-9543. (c) Dick, A. R.; Hull, K. L.; Sanford, M. S. J. Am. Chem. Soc. 2004, 126, 2300-2301. 
${ }^{23}$ Kleiman, J.P.; Dubeck M. J. Am. Chem. Soc. 1963, 85, 1544-1545.

${ }^{24}$ Cope, A. C.; Siekman, R. W. J. Am. Chem. Soc. 1965, 87, 3272-3273.

${ }^{25}$ Ananikov, V. P. ACS Catal. 2015, 5, 1964-1971.

${ }^{26}$ Daugulis, O.; Roane, J.; Tran, L. D. Acc. Chem. Res. 2015, 48, 1053-1064.

27 (a) Tang, H.; Zhou, B.; Huang, X.-R.; Wang, C.; Yao, J.; Chen, H. ACS Catal. 2014, 4, 649-656. (b) Kuhl, N.; Hopkinson, M. N.; Wencel-Delord, J.; Glorius, F. Angew. Chem. Int. Ed. 2012, 51, 10236-10254.

${ }^{28}$ Yu, L.; Chen, X.; Liu, D.; Hu, L.; Yu, Y.; Huang, H.; Tan, Z.; Gui, Q. Adv. Synth. Catal. 2018, 360, 1346-1351.

${ }^{29}$ Yan, S.-Y.; Liu, Y.-J.; Liu, B.; Liu, Y.-H.; Shi, B.-F. Chem. Commun. 2015, 51, 40694072 .

${ }^{30}$ Yamaguchi, J.; Muto, K.; Itami, K. Eur. J. Org. Chem. 2013, 2013, 19-30.

${ }^{31}$ Shiota, H.; Ano, Y.; Aihara, Y.; Fukumoto, Y.; Chatani, N. J. Am. Chem. Soc. 2011, $133,14952-14955$.

32 (a) Aihara, Y.; Chatani, N. J. Am. Chem. Soc. 2014, 136, 898-901. (b) Li, M.; Dong, J.; Huang, X.; Li, K.; Wu, Q.; Song, F.; You, J. Chem. Commun. 2014, 50, 3944-3946. (c) Zhao, S.; Liu, B.; Zhan, B.-B.; Zhang, W.-D.; Shi, B.-F. Org. Lett. 2016, 18, 45864589 .

33 (a) Liu, Y.-J.; Liu, Y.-H.; Yan, S.-Y.; Shi, B.-F. Chem. Commun. 2015, 51, 6388-6391. (b) Luo, F.-X.; Cao, Z.-C.; Zhao, H.-W.; Wang, D.; Zhang, Y.-F.; Xu, X.; Shi, Z.-J. Organometallics 2017, 36, 18-21.

34 (a) Song, W.; Lackner, S.; Ackermann, L. Angew. Chem. Int. Ed. 2014, 53, 2477-2480. (b) Aihara, Y.; Chatani, N. J. Am. Chem. Soc. 2013, 135, 5308-5311.

${ }^{35}$ Zhan, B.-B.; Liu, Y.-H.; Hu, F.; Shi, B.-F. Chem. Commun. 2016, 52, 4934-4937.

${ }^{36}$ Cheng, Y.; Wu, Y.; Tan, G.; You, J. Angew. Chem. Int. Ed. 2016, 55, 12275-12279. 
${ }^{37}$ Cong, X.; Li, Y.; Wei, Y.; Zeng, X Org. Lett. 2014, 16, 3926-3929.

${ }^{38}$ Kelly, T. R.; Xie, R. L. J. Org. Chem. 1998, 63, 8045-8048.

39 (a) Chen, X.; Hao, X.; Yu, J.-Q. J. Am. Chem. Soc. 2006, 128, 6790-6791. (b) Zhang, L.; Liu, Z.; Li, H.; Fang, G.; Barry, B. D.; Belay, T. A.; Bi, X.; Liu, Q. Org. Lett. 2011, 13, 6536-6539. (c) Liu, J.; Chen, G.; Tan, Z. Adv. Synth. Catal. 2016, 358, 1174 -1194.

${ }^{40}$ (a) Goossen, L. J.; Thiel, W. R.; Rodriguez, N.; Linder, C.; Melzer, B. Adv. Synth. Catal. 2007, 349, 2241-2246. (b) Cahiez, G.; Moyeux, A.; Gager, O.; Poizat, M. Adv. Synth. Catal. 2013, 355, 790-796.

${ }^{41}$ Williams, A. C. Nickel Catalyzed C-H Activation. In $C-H$ Bond Activation in Organic Synthesis; Li, J. J.; CRC Press: Boca Raton, FL, 2015; pp 113-144.

42 (a) Crawford, J. M.; Shelton, K. E.; Reeves, E. K.; Sadarananda, B. K.; Kalyani, D. Org. Chem. Front. 2015, 2, 726-729. (b) Yang, K.; Wang, P.; Zhang, C.; Kadi, A. A.; Fun, H.-K.; Zhang, Y.; Lu, H. Eur. J. Org. Chem. 2014, 2014, 7586-7589.

${ }^{43}$ Brown, B. R.; D. Phil, M. A. Q. Rev. Chem. Soc. 1951, 5, 131-146.

${ }^{44}$ Simmons, E. M.; Hartwig, J. F. Angew. Chem., Int. Ed. 2012, 51, 3066-3072.

${ }^{45}$ Gottlieb, H. E.; Kotlyar, V.; Nudelman, A. J. Org. Chem. 1997, 62, 7512-7515.

${ }^{46}$ Grigorjeva, L.; Daugulis, O. Org. Lett. 2015, 17, 1204-1207.

${ }^{47}$ Khan, B.; Khan, A. A.; Kant, R.; Koley, D. Adv. Synth. Catal. 2016, 358, 3753-3758.

${ }^{48}$ Roane, J.; Daugulis, O. A. J. Am. Chem. Soc. 2016, 138, 4601-4607.

${ }^{49}$ Mhaske, P. C.; Shelke, S. H.; Raundal, H. N.; Jadhav, R. P. Journal of Korea Chemical Society, 2014, 58, 62-67.

${ }^{50}$ Zhang, F.; Greany, M. F. Angew. Chem. Int. Ed. 2010, 49, 2768-2771.

${ }^{51}$ (a) Weltin, D.; Picard, V.; Aupeix, K.; Varin, M.; Oth, D.; Marchal, J.; Dufour, P.; Bischoff, P. Int. J. Immunopharmac. 1995, 17, 265-271. (b) Harayama, T.; Akamatsu, H.; Okamura, K.; Miyagoe, T.; Akiyama, T.; Abe, H.; Takeuchi, Y. J. Chem. Soc., Perkin Trans. 1. 2001, 0, 523-528. (c) Harayama, T.; Akiyama, T.; Nakano, Y.; Shibaike, K.; Akamatsu, H.; Hori, A.; Abe, H.; Takeuchi, Y. Synthesis, 2002, 2, 237 241. 
52 (a) S. D. Shnyder, P. A. Cooper, N. J. Millington, J. H. Gill, M. C. Bibby, J. Nat. Prod. 2008, 71, 321-324. (b) Hesse M. In: Wallimann PM, Kisakurek MV, eds. Alkaloids Nature's Curse or Blessing? Zurich: Wiley-VCH; 2002.

${ }^{53}$ Weltin, D.; Holl, V.; Hyun, J. W.; Dufour, P.; Marchal, J.; Bischoff, P. Int. J. Radiat. Biol. 1997, 72, 685-692.

${ }^{54}$ Huck, B. R.; Chen, X.; Deselm, L. C.; Jones, C. C. V.; Karra, S. R.; Xiao, Y.; Goutopoulos, A.; Sutton, A. E. Protein Kinase Inhibitors and Use Thereof U.S. Pat. Appl. Publ., 20110053906 Mar. 3, 2011

${ }^{55}$ Rivaud, M.; Mendoza, A.; Sauvain, M.; Valentin, A.; Jullian, V. Bioorg. Med. Chem. 2012, 20, 4856-4861.

${ }^{56}$ Tan, G. T.; Pezzuto, M. J.; Kinghorn, A. D. J. Nat. Prod. 1991, 54, 143-154.

${ }^{57}$ Ishikawa, T. Med. Res. Rev. 2000, 21, 61-72.

${ }^{58}$ Guerette, M.; Najari, A.; Maltais, J.; Pouliot, J.-R.; Dufresne, S.; Simoneau, M.; Besner, S.; Charest, P.; Leclerc, M. Adv. Energy. Matter. 2016, 6, 1502094.

${ }^{59}$ (a) Ferraccioli, R.; Carenzi, D.; Rombola, O.; Catellani, M. Org. Lett. 2004, 6, 47594762. (b) Kuwata, Y.; Sonoda, M.; Tanimori, S. J. Heterocycl. Chem. 2017, 54, 16451651. (c) Karra, S.; Xiao, Y.; Chen, X.; Liu-Bujalski, L.; Huck, B.; Sutton, A.; Goutopoulos, A.; Askew, B.; Josephson, K.; Jiang, X.; Shutes, A.; Shankar, V.; Noonan, T.; Garcia-Berrios, G.; Dong, R.; Dhanabal, M.; Tian, H.; Wang, Z.; Clark, A.; Goodstal, S. Bioorg. Med. Chem. Lett. 2013, 23, 3081-3087.

${ }^{60}$ (a) Karthikeyan, J.; Cheng, C.-C. Angew. Chem. 2011, 123, 10054-10057. (b) Yedage, S. L.; Bhanage, B. M. J. Org. Chem. 2016 81, 4103-4111. (c) Karthikeyan, J.; Haridharan, R.; Cheng, C.-C. Angew. Chem. Int. Ed. 2012, 51, 12343-12347. (d) Zhang, T.-Y.; Lin, J.-B.; Li, Q.-Z.; Kang, J.-C.; Pan, J.-L., Hou, S.H.; Chen, C.; Zhang, S.-Y. Org. Lett. 2017, 19, 1764-1767.

${ }^{61}$ Mandal, A.; Selvakumar, J.; Dana, S.; Mukherjee, U.; Baidya, M. Chem. Eur. J. 2018, $24,3448-3454$. 
${ }^{62} \mathrm{Li}$, D.; Xu, N.; Zhang, Y.; Wang, L. Chem. Commun. 2014, 50, 14862-14865.

${ }^{63}$ Li, L.; Mathieu, M.-C.; Denis, D.; Therien, A. G.; Wang, Z. Bioorg. Med. Chem. Lett. 2011, 21, 734-737.

${ }^{64}$ Kovarova, A.; Svoboda, J.; Novotna, V.; Glogavora, M.; Salamonczyk, M.; Pociecha, D.; Gorecka, E. Liq. Cryst. 2010, 37, 1501-1513.

${ }^{65}$ (a) Takamatsu, K.; Hirano, K.; Miura, M. Angew. Chem. Int. Ed. 2017, 56, 5353-5357.

(b) Travieso-Puente, R.; Budzak, S.; Chen, J.; Stacko, P.; Jastrzebski, J. T. B. H.; Jacquemin, D.; Otten, E. J. Am. Chem. Soc. 2017, 139, 3328-3331. (c) Kwan, E. E.; Zeng, Y.; Besser, H. A.; Jacobsen, E. N. Nat. Chem. 2018, 10, 917-923.

66 Honeycutt, A. P.; Hoover, J. M. ACS Catal. 2017, 7, 4597-4601.

67 Fier, P. S.; Hartwig, J. F. Science, 2013, 342, 956-960.

68 Taylor, E. C.; Zhou, P. Org. Prep. Proced. Int. 1997, 29, 221-223.

69 Maltais, F.; Bemis, G.; Wang, T.; Jimenez, J.-M.; Knegtel, R.; Davis, C.; Fraysse, D. Kinase Inhibitors. U.S. Pat. Appl. Publ., 20090124602, May 14, 2009.

70 Wang, H.; Zhang, S.; Wang, Z.; He, M.; Xu, K. Org. Lett. 2016, 18, 5628-5631.

71 Shibata, K.; Chatani, N. Org. Lett. 2014, 16, 5148-5151.

72 (a) Castro, L. C. M.; Chatani, N. Chem. Lett. 2015, 44, 410- 421. (b) Zhao, S.; Liu, B.; Zhan, B.-B.; Zhang, W.D.; Shi, B.-F. Org. Lett. 2016, 18, 4586-4589. (c) Chatani, N. Top. Organomet. Chem. 2015, 56, 19-46.

${ }^{73}$ Omer, H. M.; Liu, P. J. Am. Chem. Soc. 2017, 139, 9909-9920.

74 (a) Zheng, B.; Tang, F.; Luo, J.; Schultz, J. W.; Rath, N. P.; Mirica, L. M. J. Am. Chem. Soc. 2014, 136, 6499-6504. (b) Schultz, J. W.; Fuchigami, K.; Zheng, B.; Rath, N. P.; Mirica, L. M. J. Am. Chem. Soc. 2016, 138, 12928-12934. (c) Camasso, N. M.; Sanford, M. S. Science 2015, 347, 1218-1220. (d) Bour, J. R.; Camasso, N. M.; Meucci, E. A.; Kampf, J. W.; Canty, A. L.; Sanford, M. S. J. Am. Chem. Soc. 2016, $138,16105-16111$. 
${ }^{75}$ Beattie, D. D.; Grunwald, A. C.; Perse, T.; Schafer, L. L.; Love, J. A. J. Am. Chem. Soc. 2018, 140, 12602-12610.

${ }^{76}$ Honeycutt, A. P.; Hoover, J. M. Org. Lett. 2018, 20, 7216-7219.

${ }^{77}$ Eschinazi, H. E. Bull. Soc. Chim. Fr. 1952, 967-969.

78 (a) Tsuji, J.; Ohno, K. Tetrahedron Lett. 1965, 6, 3969-3971. (b) Ohno, K.; Tsuji, J. J. Am. Chem. Soc. 1968, 90, 99-107.

${ }^{79}$ Goossen, L. J.; Rodríguez, H. Chem. Commun. 2004, 724-725.

${ }^{80}$ Goossen, L. J.; Paetzold, J. Angew. Chem. Int. Ed. 2004, 43, 1095-1098.

81 (a) O’Brien, E. M.; Bercot, E. A.; Rovis, T. J. Am. Chem. Soc. 2003, 125, 1049810499. (b) Kajita, Y.; Kurahashi, T.; Matsubara, S. J. Am. Chem. Soc. 2008, 130, 17226-17227.

82 (a) Poater, A.; Vummaleti, S. V. C.; Cavallo, L. Organometallics 2013, 32, 6330-6336. (b) Shiba, T.; Kurahashi, T.; Matsubara, S. J. Am. Chem. Soc. 2013, 135, 13636-13639. (c) Yuan, Y.-C.; Kamaraj, R.; Bruneau, C.; Labasque, T.; Roisnel, T.; Gramage-Doria, R. Org. Lett. 2017, 19, 6404-6407.

${ }^{83}$ Morioka, T.; Nishizawa, A.; Furukawa, T.; Tobisu, M.; Chatani, N. J. Am. Chem. Soc. 2017, 139, 1416-1419.

${ }^{84}$ Kajita, Y.; Matsubara, S.; Kurahashi, T. J. Am. Chem. Soc. 2008, 130, 6058-6059.

${ }^{85}$ Zhao, T.-T.; Xu, W.-H.; Zheng, Z.-J.; Xu, P.-F.; Wei, H. J. Am. Chem. Soc. 2018, 140, 586-589.

${ }^{86}$ Chuprakov, S.; Hwang, F. W.; Gevorgyan, V. Angew. Chem. In. Ed 2007, 46, $4757-$ 4759.

${ }^{87}$ Horneff, T.; Chuprakov, S.; Chernyak, N.; Gevorgyan, V. Fokin, V. V. J. Am. Chem. Soc. 2008, 130, 14972-14974.

${ }^{88}$ Miura, T.; Yamauchi, M.; Murakami, M. Org. Lett. 2008, 10, 3085-3088. 
${ }^{89}$ Yamauchi, M.; Morimoto, M.; Miura, T.; Murakami, M. J. Am. Chem. Soc. 2010, 132, 54-55.

${ }^{90}$ Miura, T.; Morimoto, M.; Yamauchi, M.; Murakami, M. J. Org. Chem. 2010, 75, 5359-5362.

${ }^{91}$ Miura, T.; Nishida, Y.; Morimoto, M.; Yamauchi, M.; Murakami, M. Org. Lett. 2011, 13, 1429-1431.

${ }^{92}$ Thorat, V. H.; Upadhyay, N. S.; Murakami, M.; Cheng, C.-H. Adv. Synth. Catal. 2018, 360, 284-289.

${ }^{93}$ Balakrishnan, M. H.; Sathriyan, K.; Mannathan, S. Org. Lett. 2018, 20, 3815-3818.

${ }^{94}$ Ramesh, V. V. E.; Kale, S. S.; Kotmale, A. S.; Gawade, R. L.; Puranik, V. G.; Rajamohanan, P. R.; Sanjayan, G. J. Org. Lett. 2013, 15, 1504-1507.

${ }^{95}$ Xin, H.; Gao, Y.; Xiang, H.; Chen, D.; He, Y.; You, Q. J. Heterocyclic Chem. 2013, 50, 169-174.

${ }^{96}$ Yan, Y.; Li, H.; Niu, B.; Zhu, C.; Chen, T.; Liu, Y. Tetrahedron Lett. 2016 , 57, 41704173.

${ }^{97}$ Kwon, H. Y.; Lee, S. Y.; Lee, B. Y.; Shin, D. S.; Chung, Y. K. Dalton Trans. 2004, 0, 921-928.

${ }^{98}$ Yuan, Y.-C.; Kamaraj, R.; Bruneau, C.; Labasque, T.; Roisnel, T.; Gramage-Doria, R. Org. Lett. 2017, 19, 6404-6407.

${ }^{99}$ Yamamoto, T.; Kohara, T.; Yamamoto, A.; Bull. Chem. Soc. Jpn. 1981, 54, 2161-2168.

${ }^{100}$ APEX3 is a Bruker AXS crystallographic software package for single crystal data collection, reduction and preparation.

${ }^{101}$ Sheldrick, G. M., SHELXL-2014, Crystallographic software package, Bruker AXS, Inc., Madison, Wisconsin, USA. 
${ }^{102} \mathrm{R}_{1}=\sum\left(\left\|\mathrm{F}_{\mathrm{O}}|-| \mathrm{F}_{\mathrm{C}}\right\|\right) / \sum\left|\mathrm{F}_{\mathrm{O}}\right|, \mathrm{wR}_{2}=\left[\Sigma\left[\mathrm{w}\left(\mathrm{F}_{\mathrm{O}}{ }^{2}-\mathrm{F}_{\mathrm{c}}{ }^{2}\right)^{2}\right] / \sum\left[\mathrm{w}\left(\mathrm{F}_{\mathrm{O}}{ }^{2}\right)^{2}\right]\right]^{1 / 2}, \mathrm{R}_{\text {int. }}=\Sigma \mid \mathrm{F}_{\mathrm{O}}{ }^{2}$

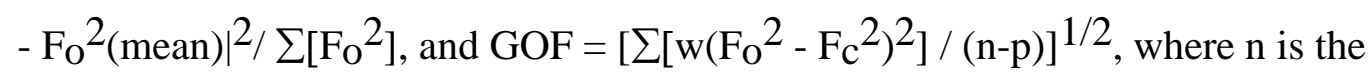
number of reflections and $\mathrm{p}$ is the total number of parameters which were varied during the last refinement cycle.

${ }^{103}$ International Tables for X-ray Crystallography (1974). Vol. IV, p. 55. Birmingham: Kynoch Press. (Present distributor, D. Reidel, Dordrecht.).

${ }^{104}$ Baur, A.; Bustin, K. A.; Aguilera, E.; Petersen, J. L.; Hoover, J. M. Org. Chem. Front. 2017, 4, 519-524. 